Escuela Técnica

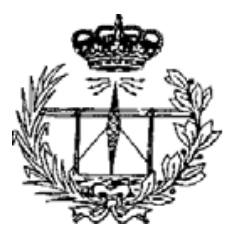

TESIS DOCTORAL

\title{
ANTENAS INTEGRADAS PARA TERMINALES MÓVILES DE TERCERA GENERACIÓN.
}

Autora: Marta Martínez Vázquez

Director: David Sánchez Hernández

Tutor: Juan Vicente Balbastre Tejedor

VALENCIA, 2003 

Lo importante no es saber, sino tener el teléfono del que sabe.

Les Luthiers

Desde el momento en que cogí su libro me cai al suelo rodando de risa. Algún día espero leerlo.

Groucho Marx 

Llegado este momento quisiera dar las gracias a todos aquellos que, de cerca o de lejos, han contribuido a que esta tesis se haya hecho realidad. Sin vuestro apoyo, vuestra ayuda y vuestros consejos nunca lo habría conseguido.

En primer lugar he de dar las gracias al Dr. David Sánchez por confiar en mí desde el primer momento, ofreciéndose a dirigir esta tesis... aunque fuera a distancia, por correspondencia y por teléfono. Al Dr. Juan Vicente Balbastre he de agradecer que se ofreciera voluntario para la ingrata tarea de tutor en la UPV. Gracias por su inestimable ayuda, y por aguantar estoicamente todas mis quejas. Al Dr. Felipe Peñaranda le agradezco que compartiera conmigo su despacho y sus "Lacasitos" durante un curso, y que me sirviera de fuente de información para todo lo referente al arduo proceso de realizar una tesis. Y a toda la gente con la que coincidí en el GCM. A todos vosotros os agradezco no sólo vuestra ayuda, sino también los buenos ratos que pasamos y vuestra amistad.

Mi agradecimiento también a la Fundación Pedro Barrié de la Maza, no sólo por concederme una beca para un año de estancia de investigación en Alemania, sino también por el cuidado con que tratan a sus becarios, más allá de los aspectos académicos. A ellos y a los demás becarios, gracias por todo.

A la dirección del IMST GmbH he de agradecer su apoyo, sin el cual nunca habría podido terminar esta tesis. En especial, al Dr. Dirk Heberling le debo haber podido realizar este trabajo en el departamento de Antenas y CEM del IMST. Espero no haberle dado motivos para arrepentirse de su corazonada al invitarme a unirme a su equipo. Lo que en principio estaba pensado como una estancia de investigación de doce meses se ha alargado hasta hoy... iу ya son casi cuatro años! Nunca habría resistido tanto tiempo si no fuera por el excelente ambiente que reina en el Equipo de Antenas, compuesto no sólo por magníficos profesionales, sino por bellísimas personas. Andreas, Lutz, Martin B., Martin G., Matthias G., Matthias S., Melanie, Michael, Oliver, Rens, Roman, Stefan und Sybille, danke für Eure Unterstützung, Eure Freundschaft und die wunderbare Arbeitsklima! Ich möchte auch allen anderen Mitarbeiter der Abteilung Antennen \& EMVU für Ihre Hilfe und Ihre Kollegialität danken.

A la Mafia le debo el haber podio sobrellevar tanto tiempo en Alemania, a pesar del mal tiempo y los horarios demenciales. Nunca olvidaré los numerosos fines de semana en el Altstadt de Dusseldorf: las horas y horas pasadas en el Sutton's o los cafés de los Domingos. Gracias por ser algo así como una familia en el exilio. 
Mi agradecimiento también a todos los amigos, en Valencia, en Alemania, y en muchos otros lugares, que al preguntar continuamente: “¿Cómo va la tesis?” evitaron que ésta cayera en el olvido. ¡Ahora sí, esta vez parece que estará lista a finales de Julio!

Quiero agradecer en particular el apoyo incondicional de mis padres, Antonio e Isabel. Gracias por vuestro cariño y ejemplo durante todos estos años. Sin olvidar a mis hermanos Bruno y Marcos, a Sarela, mi Bicho preferido, y al resto de mi familia. A ellos quiero dedicar este trabajo.

Y a Ángel, sin ti nunca habría sobrevivido a tantos fines de semana frente al portátil. Gracias por haber estado junto a mí durante estos largos meses, por tu infinita paciencia para aguantarnos a mí y mis quejas, y ayudarme a superar los momentos de desesperación.

Kamp-Lintfort, Alemania, Abril 2003. 
Para competir en el mercado, los modernos terminales de comunicaciones móviles deben cumplir requisitos muy exigentes. Deben ser capaces de operar en diferentes redes, con distintos estándares, (GSM 900, GSM 1800, PCS, UMTS...), y satisfacer ciertas especificaciones de diseño como ser pequeños y ligeros, atractivos para el usuario y de bajo coste de producción, tanto para materiales como para su montaje. La solución preferida en la actualidad, en lo que a antenas para terminales móviles se refiere, es el uso de parches metálicos radiantes, con resonancias múltiples, para cubrir así las diferentes bandas de frecuencias. Estos parches se pueden adaptar fácilmente a la forma del terminal, y pueden ser por lo tanto integradas dentro de la cubierta posterior. Esta solución presenta varias ventajas: los diseñadores no deben preocuparse por la antena a la hora de definir la forma externa del terminal, éste es más robusto, pues no hay ningún elemento radiante externo que pueda romperse, las antenas pueden ser producidas con menor coste. Sin embargo, cada vez más bandas de frecuencia deben ser cubiertas por antenas contenidas en un volumen que disminuye rápidamente. Por lo tanto, la miniaturización es clave para el diseño de las antenas.

En este trabajo se presentan algunas investigaciones acerca de la operación multibanda de antenas parche para terminales de comunicaciones móviles. Primero se estudió el efecto de integrar un filtro espolón en una antena microtira, para obtener un modo de operación dual. Una vez validado el concepto, y para resolver el problema del escaso ancho de banda inherente a las antenas impresas, se extendió el estudio a las antenas tipo PIFA (Planar Inverted-F Antenna). Se analizaron también diferentes parámetros, como la longitud del terminal o la inclinación de la antena, para determinar su influencia sobre el funcionamiento de la antena.

Se estudiaron también diferentes conceptos, con el fin de desarrollar parches de banda dual, GSM/DCS. Estas estructuras, basadas en la PIFA, consisten en un parche metálico cortocircuitado con dos modos resonantes. A partir de ellas, y añadiendo otro parche parásito también cortocircuitado, se obtienen antenas de triple banda, para cubrir el las frecuencias reservadas para PCS. Finalmente, se añade una ranura en el parche principal, para poder operar también en UMTS.

Para terminar, se discute el efecto de los materiales usados para fabricar el soporte de las antenas y la cubierta trasera del terminal, en términos de pérdida de adaptación y de eficiencia. 

To be competitive on the market, the latest personal communications handsets must face challenging demands. They have to operate within different networks with different standards (GSM, DCS, PCS, UMTS...), and still fulfil design constraints such as small size, weight, and user acceptance, while keeping their low cost with respect to materials and mounting. The preferred solution nowadays regarding handset antennas are metallic patch structures with multiple resonances, covering different bands, which are easily adapted to the shape of the handset and can therefore be integrated within the back cover. This solution has several advantages: fashion designers do not anymore have to take the antenna into account, the phone becomes more robust, as there are no external radiating elements that could break of, and the antenna can be produced in a more cost-effective way. Because more frequency bands must be covered by antennas that are contained in a volume that becomes ever smaller, miniaturization is the keyword for the mobile handset antenna designer.

In this work, some investigations were carried out concerning multiband operation of patch antennas for mobile communications terminals. First, the effect of embedding a spurline filter into a microstrip antenna to obtain dual-band operation was studied and its concept validated. Then, to overcome the bandwidth problem inherent to microstrip patches, a PIFA concept was chosen. Different parameters, such as terminal length and antenna inclination, were investigated, to assert their influence upon the antenna performance.

Different concepts were looked into, aiming at the development of dual-band, GSM/DCS patches. These structures consist of a single radiating shorted PIFA-like patch, with two resonant modes. Based upon these results, new radiating elements with triple band operation are also presented, covering the band reserved for PCS by using a shorted, parasitic plate. Last, a slot was added to provide UMTS operation.

Finally, the effect of the materials used to fabricate the support of the antennas and the back cover of the handset is discussed, with regard to impedance matching and efficiency loss. 



\section{Resumen.}

Per a competir en el mercat, els moderns terminals de comunicacions mòbils deuen complir requisits molt exigents. Deuen ser capaços d'operar en diferents xarxes, amb distints estàndards, (GSM, DCS, PCS, UMTS...), i satisfer certes especificacions de disseny com ser petits i lleugers, atractius per a l'usuari i de baix cost de producció, tant per a materials com per al seu muntatge. La solució preferida en l'actualitat, en el que a antenes per a terminals mòbils es refereix, és l'ús de pegats metàl-lics radiants, amb ressonàncies múltiples, per a cobrir així les diferents bandes de freqüències. Aquests pegats es poden adaptar fàcilment a la forma del terminal, i poden ser per tant integrades dintre de la coberta posterior. Aquesta solució presenta diversos avantatges: els dissenyadors no deuen preocupar-se per l'antena a l'hora de definir la forma externa del terminal, aquest és més robust, doncs no hi ha cap element radiant externs que pugui trencar-se, les antenes poden ser produïdes amb menor cost. No obstant això, cada vegada més bandes de freqüència deuen ser cobertes per antenes contingudes en un volum que disminueix ràpidament. Per tant, la miniaturització és clau per al disseny de les antenes.

En aquest treball es presenten algunes investigacions sobre l'operació multibanda d'antenes parche per a terminals de comunicacions mòbils. Primer es va estudiar l'efecte d'integrar un filtre espolón en una antena microtira, per a obtenir una d'operació dual. Una vegada validat el concepte, i per a resoldre el problema de l'escàs ample de banda inherent a les antenes impreses, es va estendre l'estudi a les antenes tipus PIFA (Planar Inverted-F Antenna). Es van analitzar també diferents paràmetres, com la longitud del terminal o la inclinació de l'antena, per a determinar la seva influència sobre el funcionament de l'antena.

Es van estudiar també diferents conceptes, amb la finalitat de desenvolupar parches de banda dual, GSM/DCS. Aquestes estructures, basades en la PIFA, consisteixen en un parche metàl-lic curtcircuitat amb dues ressonàncies. A partir d'elles, i afegint altre parche paràsit també curtcircuitat, s'obtenen antenes de triple banda, per a cobrir el les freqüències reservades per a PCS. Finalment, s'afegeix una ranura en el parche principal, per a poder operar també en UMTS.

Per a acabar, es discuteix l'efecte dels materials usats per a fabricar el suport de les antenes i la coberta del darrere del terminal, en termes de pèrdua d'adaptació i d'eficiència. 



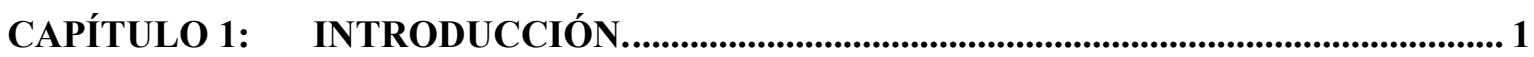

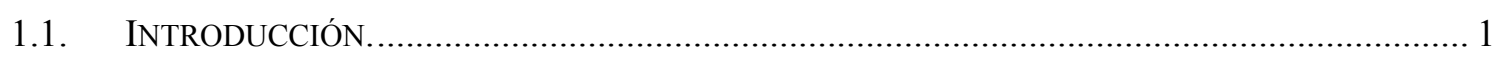

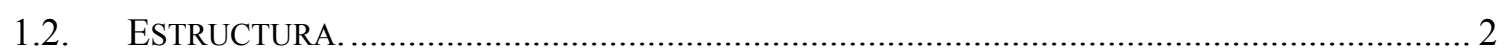

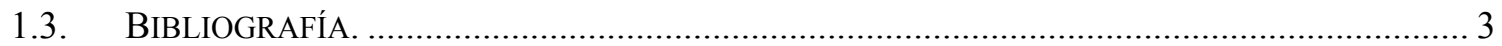

CAPITULO 2: ANTENAS PARA TERMINALES MOVILES....................................... 5

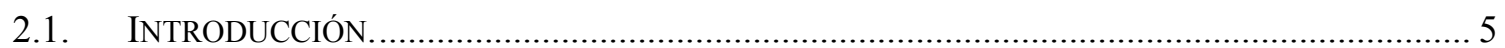

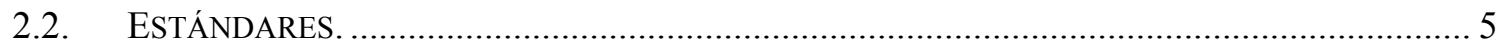

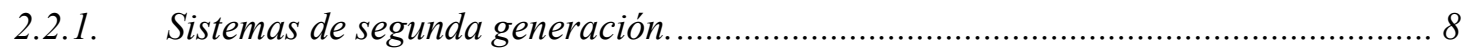

2.2.2. La tercera generación: UMTS. ……................................................................. 9

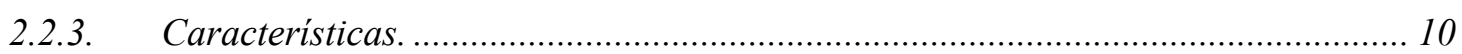

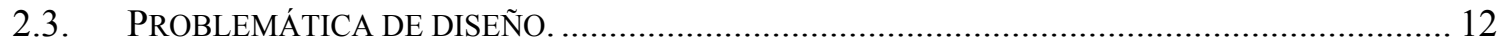

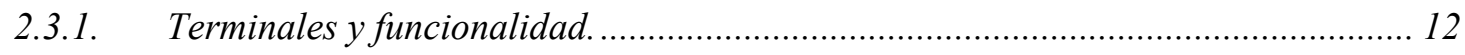

2.3.2. Efecto del plano de masa finito ............................................................................ 14

2.3.3. Restricciones en el tamaño y forma de la antena.................................................... 14

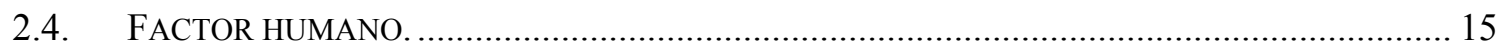

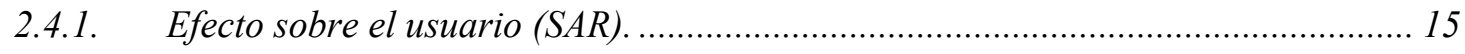

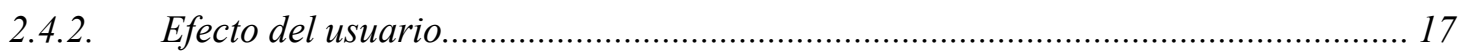

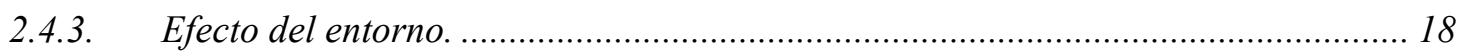

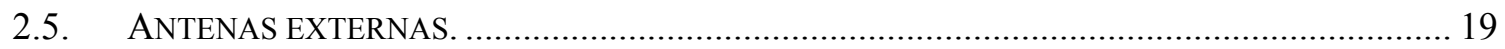

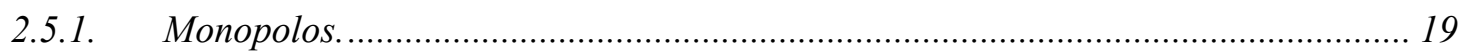

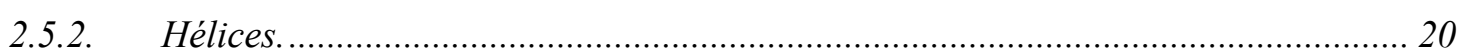

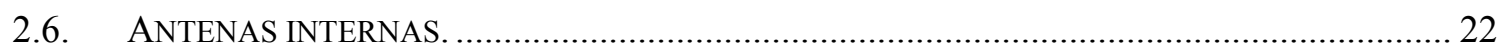

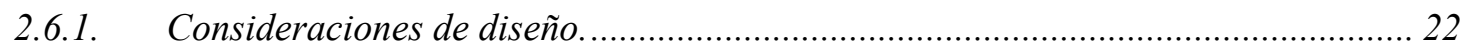

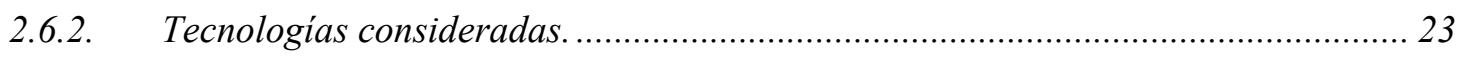

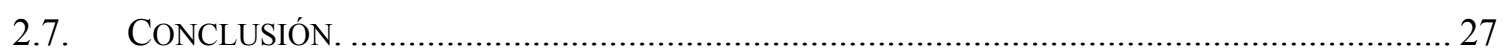

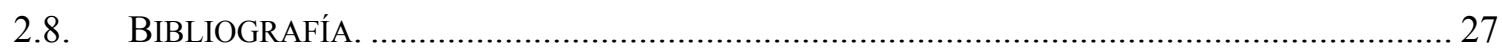

CAPÍTULO 3: INTRODUCCIÓN A LAS ANTENAS IMPRESAS. ...................................... 31

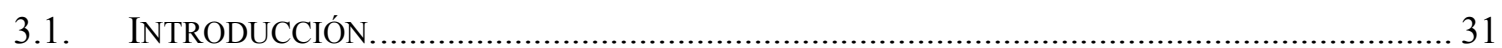

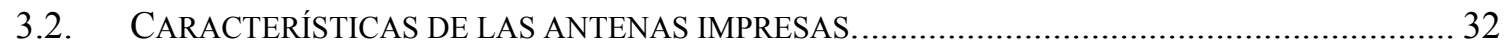

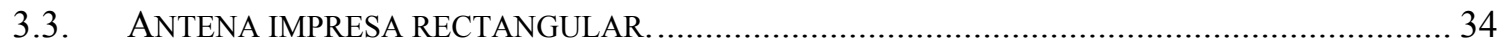




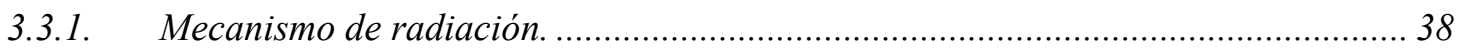

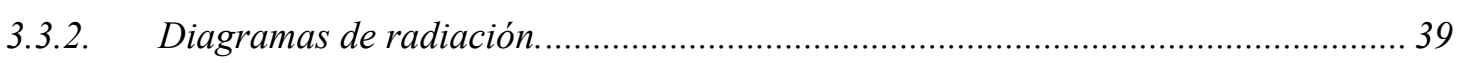

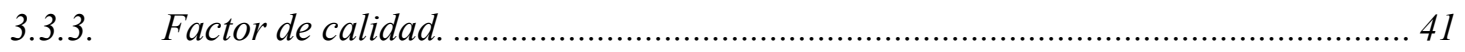

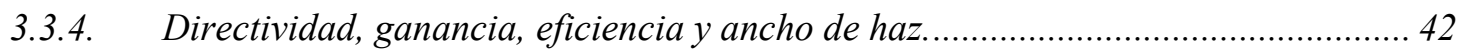

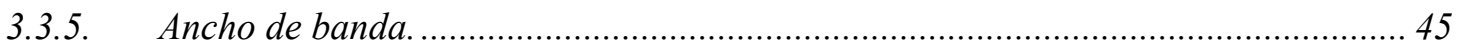

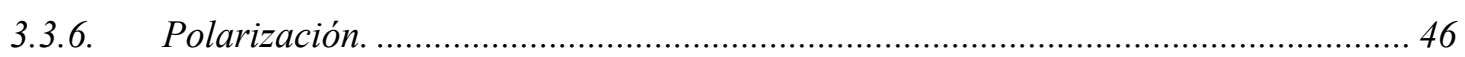

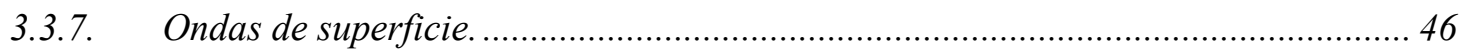

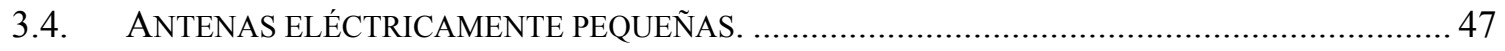

3.4.1. Límites teóricos de las antenas eléctricamente pequeñas......................................... 48

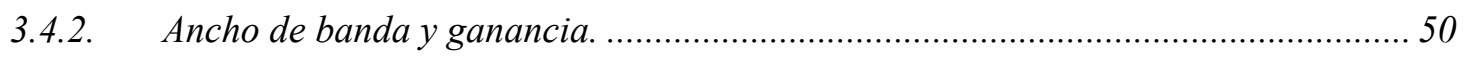

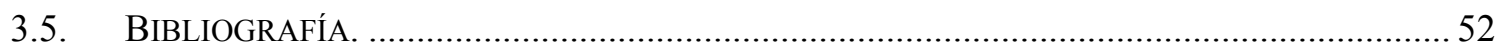

CAPÍTULO 4: MÉTODOS DE ANÁLISIS DE ANTENAS IMPRESAS..........................57

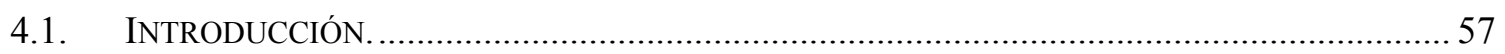

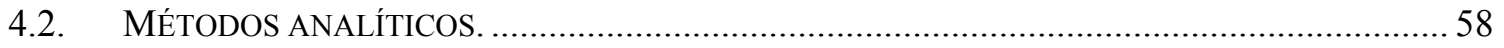

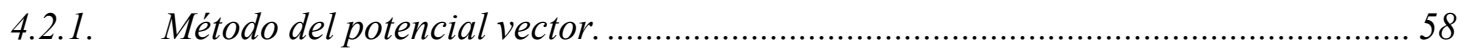

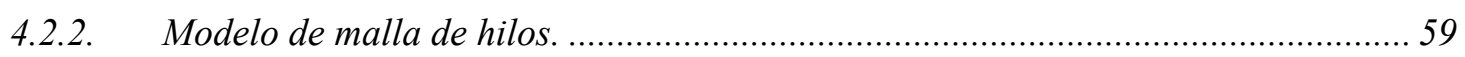

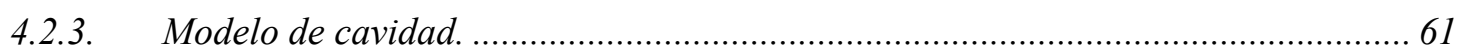

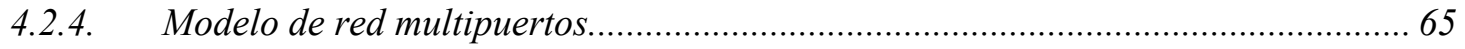

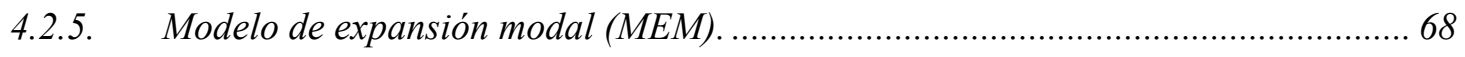

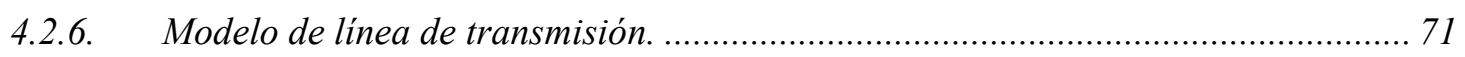

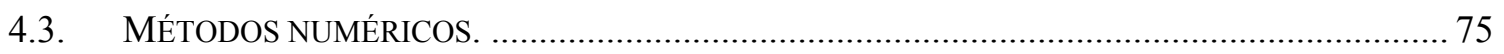

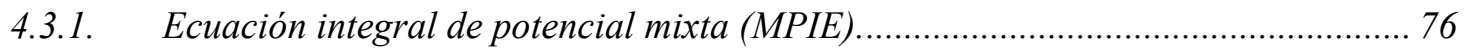

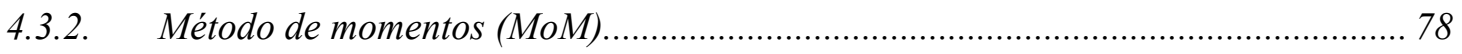

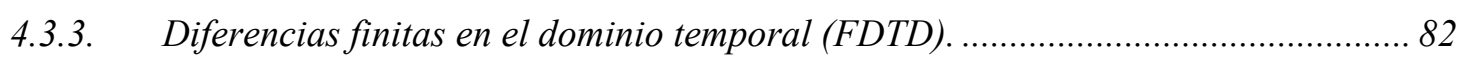

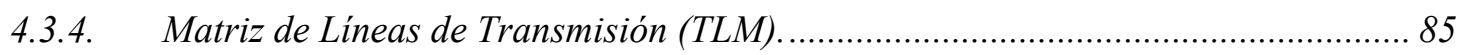

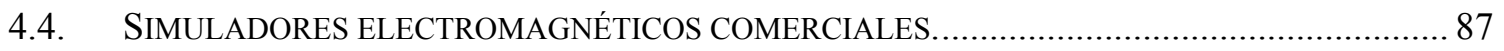

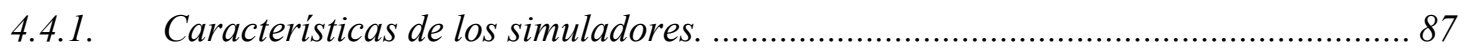

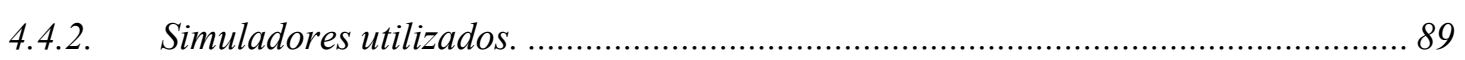

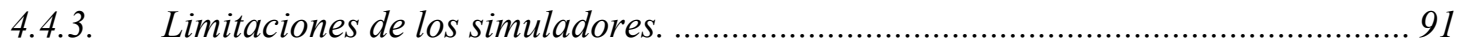

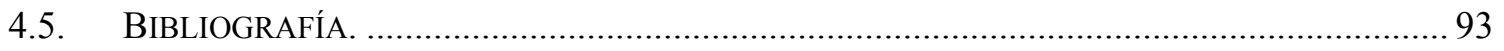

CAPÍTULO 5: ANTENAS IMPRESAS DE DOBLE BANDA........................................97

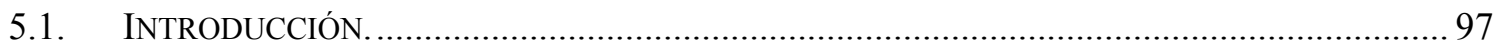

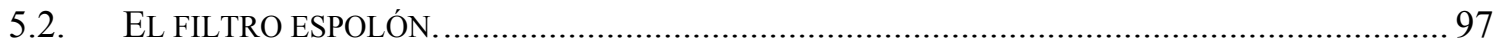

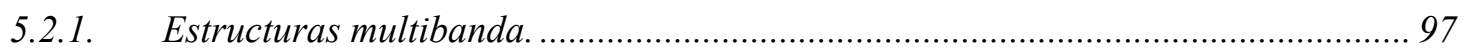

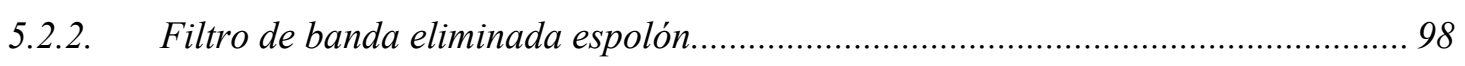

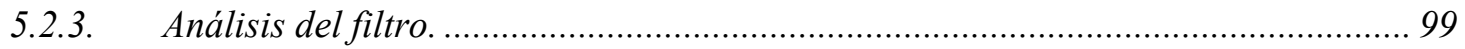

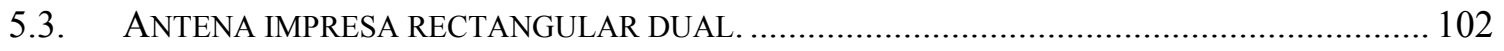




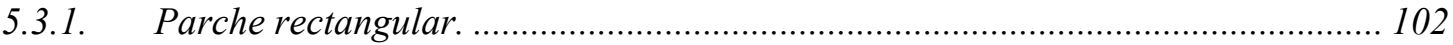

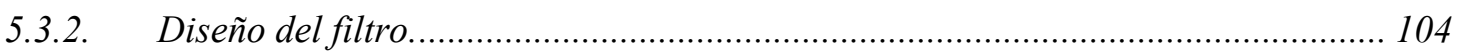

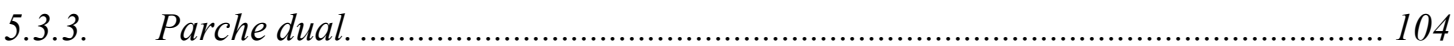

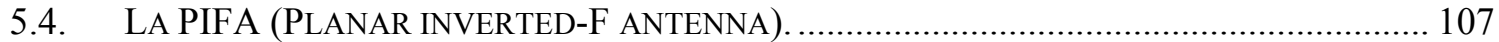

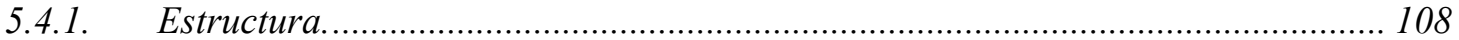

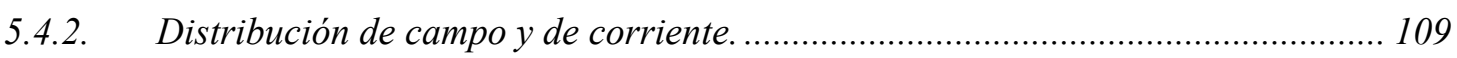

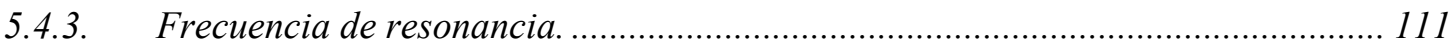

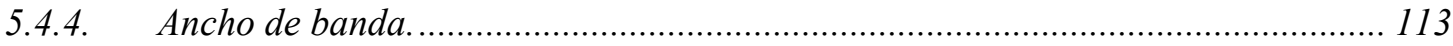

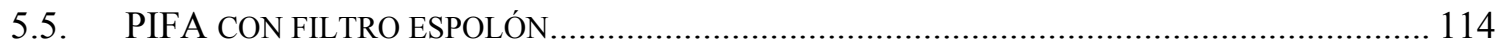

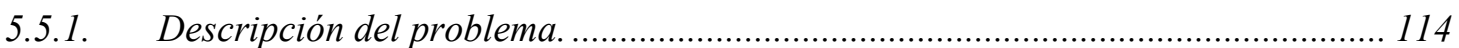

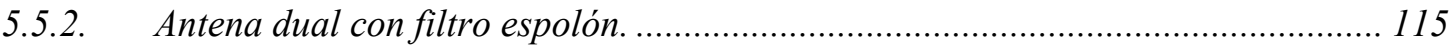

5.5.3. Efecto del tamaño del terminal. ........................................................................ 119

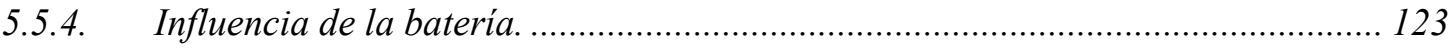

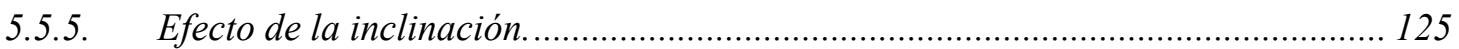

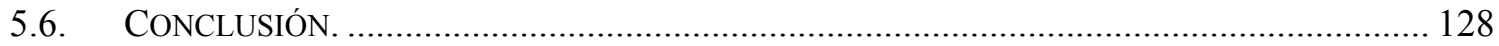

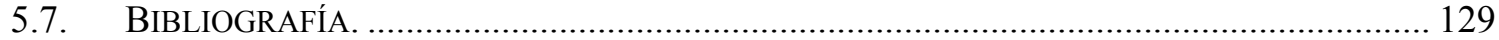

CAPÍTULO 6: ANTENAS INTEGRADAS MULTIBANDA. .............................................. 133

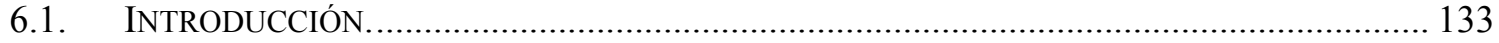

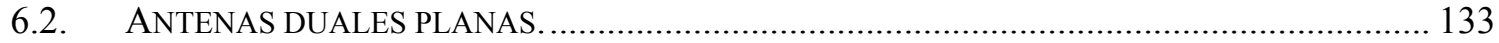

6.2.1. Antena triangular con filtro espolón................................................................... 133

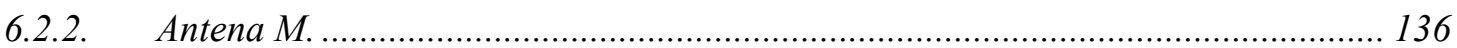

6.2.3. Antena M con conector integrado .................................................................. 139

6.2.4. Antena "ring-patch". ................................................................................... 141

6.3. ANTENAS ADAPTADAS A LA FORMA DEL TERMINAL.................................................... 145

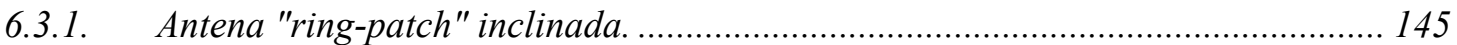

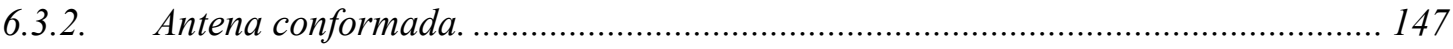

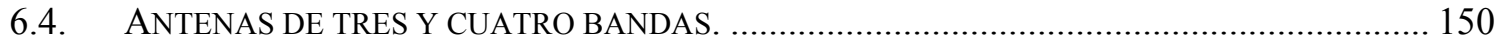

6.4.1. Antena de triple banda con parche parásito M3 ................................................. 152

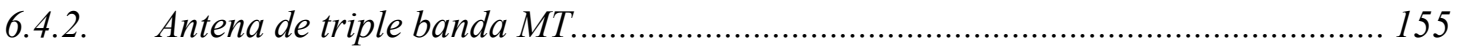

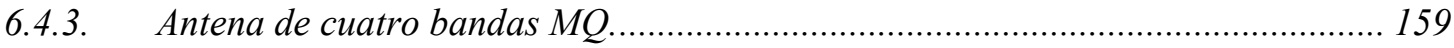

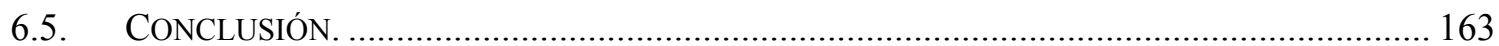

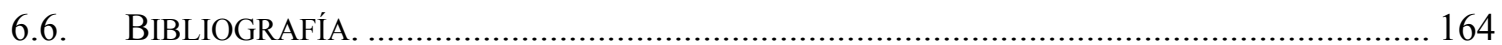

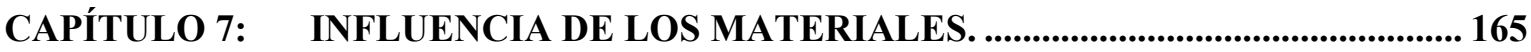

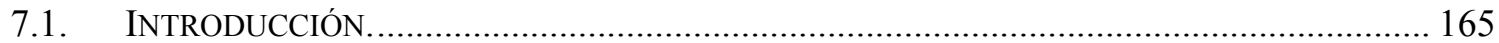

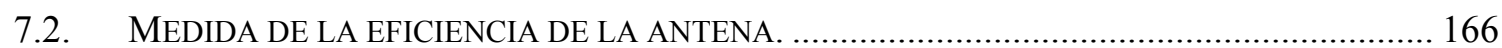

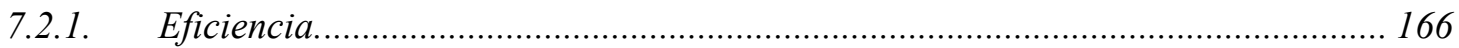

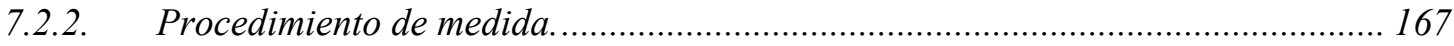




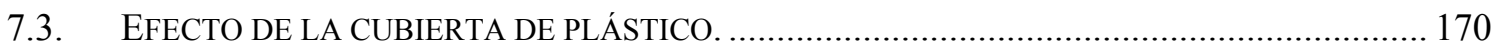

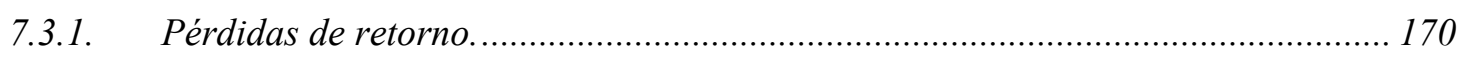

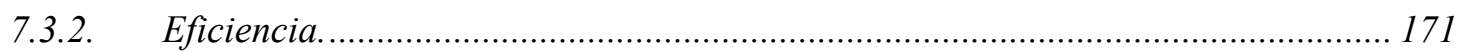

7.4. EFECTO DEL SOPORTE DIELÉCTRICO Y DE LA METALIZACIÓN....................................... 173

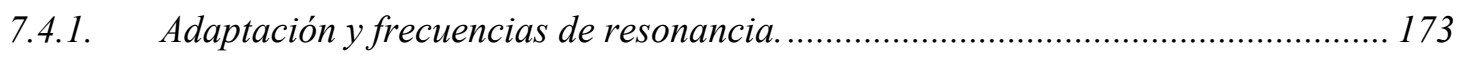

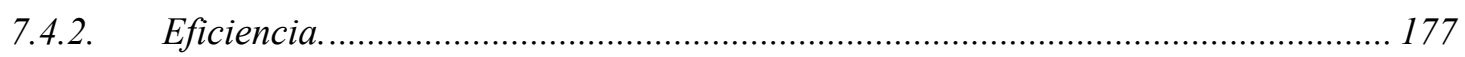

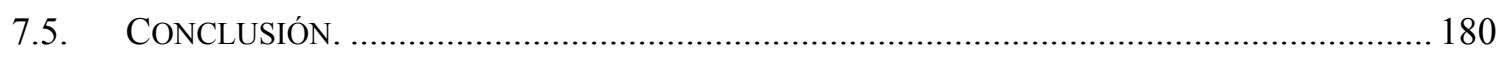

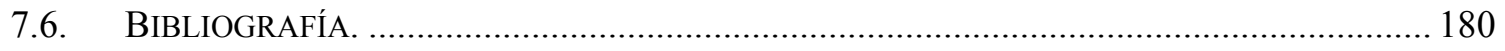

CAPÍTULO 8: CONCLUSIONES Y LÍNEAS FUTURAS. ............................................ 183

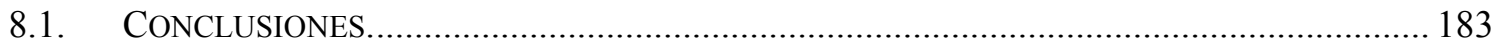

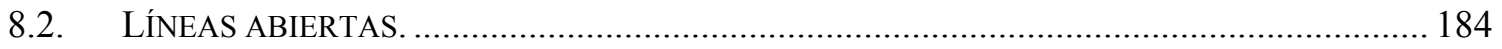

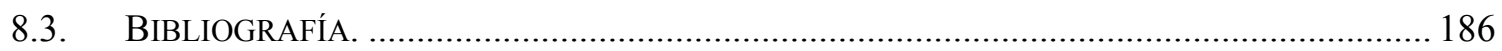

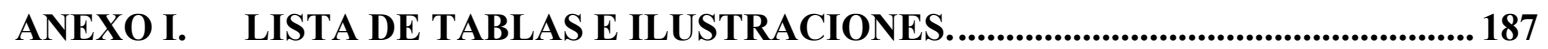

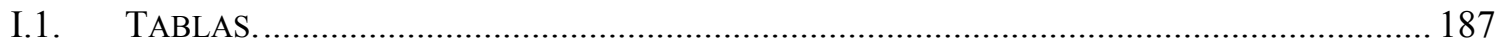

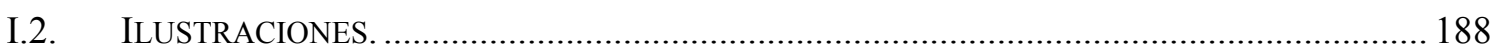

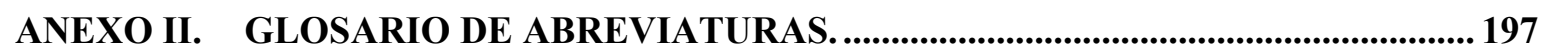

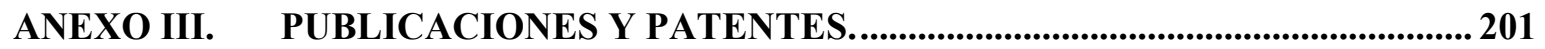

III.1. PUBLICACIONES EN REVISTAS INTERNACIONALES. ...................................................... 201

III.2. PUBLICACIONES EN CONFERENCIAS INTERNACIONALES.............................................. 201

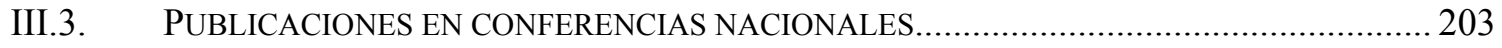

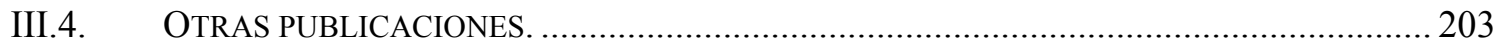

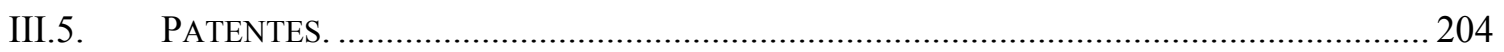




\section{Capítulo 1: INTRODUCCIÓN.}

Comenzar bien no es poco, pero tampoco mucho.

Sócrates

\subsection{INTRODUCCIÓN.}

El auge del mercado de las telecomunicaciones móviles es un fenómeno sin precedentes, sólo comparable con el de Internet. En menos de una década, los terminales de comunicaciones móviles han pasado de ser aparatos extraños y voluminosos a convertirse en objetos familiares cada vez más diminutos y con más aplicaciones. La "cultura del móvil" se ha desarrollado de tal forma que hoy en día poseer un terminal móvil parece casi imprescindible.

Para combinar la demanda de comunicaciones móviles y de aplicaciones multimedia surge el estándar UMTS, símbolo de la llamada tercera generación. Mientras se llega a un sistema único, y durante la fase de migración, son varios los estándares que cohabitan, compartiendo incluso áreas geográficas. Por esta razón se da por hecho que cualquier terminal actual es, por defecto, dual GSM/DCS, y se tiende a introducir cada vez más el estándar PCS, para responder a la demanda de los usuarios, que requieren más movilidad. También los operadores prefieren estos terminales multibanda, que les permiten aliviar sus problemas de capacidad [1.1]. Esto repercute en el diseño de los terminales, en particular de las antenas, ya que se deben cubrir simultáneamente distintas bandas de frecuencias, sin perder calidad en el enlace.

Por otra parte, la microelectrónica avanza a pasos agigantados, con lo que hoy por hoy es posible fabricar terminales cada vez más pequeños y ligeros. Esto satisface los requerimientos estéticos de fabricantes y usuarios, pero complica la labor de los diseñadores de antenas, puesto que el tamaño de éstas está determinado por criterios físicos [1.2].

De ahí que, a pesar de las resistencias de ciertos sectores, las antenas tengan un papel primordial en el diseño de los terminales, y su desarrollo deba correr paralelo al de éstos [1.3]. El monopolo, utilizado como elemento dominante en los primeros terminales de comunicaciones móviles, ha sido desterrado casi por completo, sustituido en un primer momento por las hélices. Sin embargo, se observa una clara tendencia hacia la incorporación de antenas en el interior de los terminales, para 
responder a las demandas del mercado [1.4].

Tal y como se ha mencionado, los terminales actuales son multibanda, por lo que las antenas deben cumplir este requisito, teniendo en cuenta además que el volumen destinado a la antena también disminuye con el tamaño del terminal. Surge entonces la necesidad de emplear técnicas que permitan conseguir la operación en banda dual sin incrementar el volumen total de la antena y, además, conseguir que se cumplan los requisitos de ancho de banda que se imponen en cada una de las bandas de operación.

El objetivo de esta tesis es proponer soluciones para la integración de antenas multibanda en terminales de comunicaciones móviles. Para ello se diseñarán parches radiantes resonantes a dos o más frecuencias, que corresponden a las de los principales estándares de comunicaciones móviles. Se estudiará cómo conseguir diferentes modos con un parche radiante, o una combinación de parches radiantes, parches parásitos y ranuras, utilizando para ello un único punto de alimentación.

\subsection{ESTRUCTURA.}

Contando con este Capítulo 1, de introducción, este trabajo consta de ocho capítulos.

En primer lugar, en el Capítulo 2 se da una visión general del problema, en lo referente a los estándares de comunicaciones móviles y su evolución. Se describen también las particularidades que hay que tener en cuenta en el diseño de las antenas para los terminales, ligadas tanto a la geometría del problema como la presencia del usuario. Se hace también un breve repaso de los tipos de antenas consideradas, así como de las tecnologías más comunes para su fabricación.

Teniendo en cuenta que las antenas que se desarrollarán en esta tesis son parche radiantes, basados en el principio de las antenas impresas, en el Capítulo 3 se resumen las principales características de estas últimas. En él se definen algunos conceptos que se utilizarán a lo largo de todo el trabajo. Un concepto importante que se introduce es el de antenas eléctricamente pequeñas, que englobará a todas las antenas aquí presentadas.

Para el análisis de estas estructuras se dispone de diferentes modelos analíticos, algunos de los cuales se presentan en el Capítulo 4. Sin embargo, dada la complejidad de los problemas, es necesario recurrir a métodos numéricos para su estudio. Así, en este apartado se describirán algunos de los métodos numéricos más utilizados. También se hará un repaso de las herramientas de simulación que los implementan. Se verá que, si bien estos simuladores electromagnéticos son hoy en día imprescindibles para el diseño de antenas, en especialmente aquellas integradas en terminales de comunicaciones móviles, presentan una serie de limitaciones que hay que tener en cuenta. 
En el Capítulo 5 se estudia la posibilidad de implementar estructuras radiantes de doble banda con un solo parche, para satisfacer así las necesidades de los fabricantes, que se decantan por terminales multiestándar. En este caso se pretende introducir un segundo modo resonante utilizando para ello un filtro de banda eliminada espolón. Se verá que el uso de las antenas impresas tradicionales está limitado por su gran tamaño a las frecuencias de comunicaciones móviles, así como su escaso ancho de banda. Sin embargo la combinación del filtro espolón con una antena plana en F invertida (Planar Inverted-F Antenna, PIFA) proporciona resultados interesantes. Se estudiará también cómo el alterar diferentes parámetros, como el tamaño del terminal o la posición de la antena, influye sobre el rendimiento final.

El Capítulo 6 sirve para desarrollar las ideas expuestas en el Capítulo 5. En él se presentarán diferentes estructuras radiantes de dos o más bandas, todas ellas de tamaño reducido, para satisfacer los requerimientos de diseño de los terminales móviles. Se verá cómo es posible lograr estructuras multiresonantes combinando parches activos, parches parásitos y ranuras radiantes.

El funcionamiento de las antenas consideradas estará determinado en parte por las técnicas y materiales utilizados en su fabricación. Así, en el Capítulo 7 se analizará cómo el hecho de integrar la antena en un terminal típico afecta a su rendimiento. Se tendrá en cuenta el efecto de los materiales dieléctricos, utilizados como soporte o como cubierta, sobre la adaptación de la antena y su eficiencia.

Finalmente, en el Capítulo 8 se recogen algunas conclusiones derivadas de este trabajo, y se plantean algunas líneas futuras de investigación.

\subsection{Bibliografía.}

[1.1] 'Jumping on the Band Wagon', Mobile Europe, Marzo 1999, pp. 31-32.

[1.2] Edvarsson, O., 'Does Size Really Matter?', Mobile Europe, Mayo 2000, p. 33.

[1.3] Garner, W.J. y Wu, W.L., 'Designing Antenas for Cellular Phones', Microwaves \& RF, Mayo 1999, pp. 70-76.

[1.4] Edvarsson, O. y Östervall, T., 'Now You See It...', Mobile Europe, Julio-Agosto 1997, p. 24. 



\section{Capítulo 2: ANTENAS PARA TERMINALES MOVILES.}

Dick: I'll be at 362-9296 for a while; then I'll be at 648-0024 for about fifteen minutes; then I'll be at 752-0420; and then I'll be home, at 621-4598. Yeah, right George, bye-bye.

Linda: There's a phone booth on the corner. You want me to run downstairs and get the number? You'll be passing it.

Woody Allen, Play it again, Sam.

\subsection{INTRODUCCIÓN.}

A principios de los años 90 se pensaba en los sistemas de comunicaciones móviles únicamente como en un medio para mantener conversaciones mientras el usuario se desplazaba. Sin embargo nadie pudo prever su explosión, llegándose a convertir hoy en día en un verdadero fenómeno social. Cualquiera puede acceder a servicios de voz y datos, incluso multimedia, casi en cualquier punto del planeta.

En este capítulo se pretende dar una visión de los principales estándares existentes, así como de los requisitos de diseño establecidos para los terminales. Se hace igualmente un repaso de los problemas que plantea el diseño de antenas para dichos terminales, y de las tecnologías disponibles para su implementación.

\subsection{ESTÁNDARES.}

Los terminales móviles se han convertido en dispositivos de comunicación multimedia, capaz de transmitir y recibir, además de voz, datos, imágenes fijas y video. Los más recientes se pueden conectar con las redes de área local (LANs) que permiten compartir información, y resolver tareas sin necesidad de cableado.

La cobertura global está también garantizada por los sistemas de comunicaciones vía satélite, 
aunque el inicio de estos ha sido incierto y no han alcanzado el auge esperado. Sí es una realidad la combinación de sistemas móviles terrestres con el sistema de navegación GPS, que permite la localización del usuario en cualquier punto del planeta, facilitando así el control de tráfico de personas y mercancías.

Las nuevas necesidades han fomentado el desarrollo de los sistemas móviles de tercera generación, conocidos como UMTS (Universal Mobile Telecommunication System) en Europa y IMT2000 (International Mobile Telecommunications 2000) por la Unión Internacional de Telecomunicaciones (UIT). Tras un inicio espectacular, con subastas millonarias para la concesión de las licencias de UMTS en algunos países de Europa, la implantación de estos sistemas de tercera generación ha sufrido un frenazo. Su lanzamiento comercial ha sido aplazado varias veces, debido a la falta de medios económicos y de equipos, sobre todo terminales de usuario, que cumplan con las especificaciones. Sin embargo, estos problemas deberían ser resueltos en breve, y se espera el lanzamiento comercial de UMTS en Europa en el tercer trimestre de 2003.

En la Tabla 2.1 y Tabla 2.2 se recogen algunos ejemplos de redes analógicas de primera generación $(1 \mathrm{G})$ y digitales de segunda $(2 \mathrm{G})$ y tercera $(3 \mathrm{G})$ generación, respectivamente. Se describen igualmente algunas de sus características principales.

Tabla 2.1: Ejemplos de tecnologías analógicas: redes de primera generación.

\begin{tabular}{|c|c|}
\hline Estándar & Descripción \\
\hline AMPS & $\begin{array}{l}\text { Advanced Mobile Phone System. Desarrollado por Bell Labs en los años } \\
70, \text { y lanzado comercialmente en los EE.UU. en 1983. Opera en la banda } \\
\text { de } 800 \mathrm{MHz} \text {. }\end{array}$ \\
\hline C-Netz & $\begin{array}{l}\text { La tecnología celular más antigua, localizada principalmente en } \\
\text { Alemania y Austria. Usa la banda de } 450 \mathrm{MHz} \text {. }\end{array}$ \\
\hline N-AMPS & $\begin{array}{l}\text { Narrowband Advanced Mobile Phone System. Desarrollado por } \\
\text { Motorola como una tecnología intermedia entre analógica y digital. Tiene } \\
\text { una capacidad tres veces superior a la de AMPS y opera en el rango de } \\
800 \mathrm{MHz} \text {. }\end{array}$ \\
\hline NMT450 & $\begin{array}{l}\text { Nordic Mobile Telephones } / 450 \text {. Desarrollado especialmente por Ericsson } \\
\text { y Nokia para dar servicio en los países nórdicos. Opera a } 450 \mathrm{MHz} \text { y usa } \\
\text { FDD FDMA. }\end{array}$ \\
\hline NMT900 & $\begin{array}{l}\text { Nordic Mobile Telephones/900. Actualización a } 900 \text { MHz del NMT } 450 \\
\text { desarrollado por los países nórdicos. Usa tecnología FDD FDMA. }\end{array}$ \\
\hline NTT & Nippon Telegraph and Telephone. Antiguo estándar Japonés. \\
\hline TACS & $\begin{array}{l}\text { Total Access Communications System. Desarrollado por Motorola, es } \\
\text { similar al AMPS. Denominado JTAC en Japón. Opera en el rango de } \\
\text { frecuencias de } 900 \mathrm{MHz} \text {. }\end{array}$ \\
\hline
\end{tabular}


Tabla 2.2: Ejemplos de redes digitales: redes de segunda y tercera generación.

\begin{tabular}{|c|c|}
\hline Estándar & Descripción \\
\hline CDMA/TDMA & $\begin{array}{l}\text { Tecnología inalámbrica que usa CDMA y TDMA (CDMA entre células y TDMA } \\
\text { dentro de las células). }\end{array}$ \\
\hline CDMA & $\begin{array}{l}\text { Code Division Multiple Access. Existen diferentes variantes del CDMA original, } \\
\text { conocido como IS-95. La principales son W-CDMA y CDMA/TDMA. CDMA se } \\
\text { caracteriza por su alta capacidad y un radio de células pequeño, empleando técnicas de } \\
\text { espectro ensanchado (spread-spectrum). W-CDMA es la base para 3G UMTS. }\end{array}$ \\
\hline cdmaOne & Primera generación de CDMA, de banda estrecha. \\
\hline $\operatorname{cdma} 2000$ & La nueva generación CDMA Compatible con UMTS. \\
\hline D-AMPS & $\begin{array}{l}\text { Digital AMPS, variante digital del AMPS. Por el uso de TDMA en lugar de FDMA, } \\
\text { incrementa el número de usuarios. Una infraestructura AMPS/D-AMPS puede soportar } \\
\text { el uso de teléfonos analógicos y digitales. Ambas operan en } 800 \mathrm{MHz} \text {. }\end{array}$ \\
\hline DCS & $\begin{array}{l}\text { Digital Cellular System, también denominado GSM 1800. Opera en la banda de } 1800 \\
\text { MHz. }\end{array}$ \\
\hline DECT & $\begin{array}{l}\text { Digital Enhanced Corldless Telecommunications. Estándar inalámbrico digital que } \\
\text { utiliza tecnología TDMA. }\end{array}$ \\
\hline EDGE & $\begin{array}{l}\text { Enhanced Data rate for GSM Evolution, o E-GSM. Permite usar las bandas de GSM ya } \\
\text { existentes para ofrecer servicios multimedia basados en IP y aplicaciones a una } \\
\text { velocidad máxima de } 386 \mathrm{kbps} \text {, con una media de entre } 48 \mathrm{kbps} \text { y hasta } 69.2 \mathrm{kbps} \text { en } \\
\text { buenas condiciones de radio. }\end{array}$ \\
\hline GSM & $\begin{array}{l}\text { Global System for Mobile communications. Primer estándar digital europeo, } \\
\text { desarrollado para establecer una compatibilidad celular en Europa. Su éxito se ha } \\
\text { extendido a todo el mundo. Usa la banda de } 900 \mathrm{MHz} \text {. DCS y PCS son variantes de } \\
\text { este sistema. }\end{array}$ \\
\hline Inmarsat & International Martime Satellite System. Usa satélites GEO (geoestacionarios). \\
\hline Iridium & $\begin{array}{l}\text { Mobile Satellite phone/pager. Usa TDMA para enlaces vía satélite en la banda de } 2 \\
\text { GHz. Constituyó un gran fracaso comercial. }\end{array}$ \\
\hline N-CDMA & $\begin{array}{l}\text { Narrowband CDMA, el antiguo CDMA. Usa la misma banda de frecuencia que AMPS } \\
\text { y soporta D-AMPS. }\end{array}$ \\
\hline PCS & $\begin{array}{l}\text { Personal Communications System. Opera en la banda de } 1900 \mathrm{MHz} \text {. Versión } \\
\text { americana del estándar GSM. }\end{array}$ \\
\hline $\mathrm{PDC}$ & $\begin{array}{l}\text { Personal Digital Cellular. Basado en el estándar TDMA japonés, opera en las bandas de } \\
800 \mathrm{MHz} \text { y } 1500 \mathrm{MHz} \text {. }\end{array}$ \\
\hline PHS & $\begin{array}{l}\text { Personal Handy System. Sistema japonés que ofrece servicios de alta velocidad de } \\
\text { datos y gran calidad de voz. }\end{array}$ \\
\hline TETRA & $\begin{array}{l}\text { TErrestrial Trunked RAdio (TETRA). Estándar de radio digital abierto definido por } \\
\text { ETSI a partir de las necesidades de los usuarios profesionales de radio móvil. }\end{array}$ \\
\hline UMTS & $\begin{array}{l}\text { Universal Mobile Telephone Standard. Estándar para la tercera generación de telefonía } \\
\text { celular global. Alcanza velocidades de hasta } 2 \mathrm{Mbps} \text {, usando conjuntamente TDMA y } \\
\text { W-CDMA. Opera alrededor de } 2 \mathrm{GHz} \text {. }\end{array}$ \\
\hline W-CDMA & $\begin{array}{l}\text { Wideband-CDMA. Uno de los más recientes componentes del UMTS, junto con } \\
\text { TDMA y cdma2000. }\end{array}$ \\
\hline
\end{tabular}




\subsubsection{Sistemas de segunda generación.}

\section{La familia de sistemas GSM.}

El sistema GSM (Global System for Mobile communications) fue pensado para satisfacer las necesidades de finales del siglo XX, ofreciendo un amplio abanico de servicios y facilidades de voz y datos, compatibles con las redes fijas mediante sistemas de acceso estandarizados. Junto a las nuevas funciones de roaming o itinerancia internacional o sus mayores niveles de calidad y seguridad en las comunicaciones frente a los disponibles en los sistemas analógicos, GSM proporciona toda una gama de servicios suplementarios, como identificación y desvío de llamadas, y de teleservicios, como mensajes cortos y correo electrónico [2.1]. Desde que GSM (fase 1) entró en servicio comercial en 1992, no ha dejado de evolucionar para adaptarse a nuevas necesidades.

Entre los diferentes estándares internacionales adoptados para garantizar la compatibilidad entre las redes celulares digitales de diferentes regiones y países, GSM ha venido a ser el estándar más empleado por todo el mundo. Aunque no es la respuesta definitiva a los requisitos de UMTS, dada la aceptación y el rápido crecimiento de estas redes, se ha convertido en la mejor plataforma para el desarrollo de la primera fase de implantación de los sistemas de tercera generación.

Gran parte del éxito de GSM reside en el hecho de haber sido definido como un estándar abierto, permitiendo a cada fabricante desarrollar sus propio hardware y software, siempre y cuando cumpliera los requisitos de compatibilidad. Esto, junto con un lanzamiento programado y una buena estrategia de comercialización, hizo de GSM el referente mundial en cuanto a sistemas de comunicaciones móviles digitales.

El sistema GSM ha sido adaptado para trabajar a $1800 \mathrm{MHz}$ para los sistemas europeos PCN (Personal Communication Networks), y a $1900 \mathrm{MHz}$ para los sistemas americanos PCS (Personal Communications System). Así, se han desarrollado los sistemas DCS (Digital Cellular System, también denominado GSM 1800) en Europa y PCS 1900 en EEUU, que usan la misma infraestructura y tecnología que GSM.

En Europa DCS complementa las redes GSM existentes, aumentando su capacidad y los servicios ofrecidos. Al mismo tiempo el desarrollo de la fase 2+ de GSM introdujo mayor ancho de banda para GSM 900, para dar cabida a nuevos usuarios y servicios, obteniéndose así el E-GSM o EDGE.

Debido a los retrasos en la implantación de UMTS, se desarrolló igualmente GPRS (General Packet Radio Services) como un paso intermedio, que podríamos denominar de 2,5G ("generación 2,5”). GPRS utiliza la técnica de conmutación de paquetes para optimizar la transmisión de datos, especialmente si se han de establecer frecuentes comunicaciones a gran escala con muchos puntos simultáneamente.

Además de GPRS, el uso de HSCSD (High Speed Circuit Switched Data) permite mejorar las prestaciones de los sistemas de conmutación de circuitos para obtener servicios de datos GSM con 
velocidades superiores a los 9600 bps. Para ello se utiliza multiplexación en el tiempo, TDMA (Time Division Multiple Access) y técnicas de compresión de datos.

\section{Sistemas americanos.}

Aunque GSM se encuentra también en los Estados Unidos, bajo las siglas PCS, existe también un sistema propio de primera generación, basado también en FDMA, en la banda de $800 \mathrm{MHz}$. Sobre la base del estándar analógico AMPS (Advanced Mobile Phone System) se desarrolló D-AMPS (Digital APMS), que permite ofrecer en una misma red servicios analógicos y digitales. Los terminales D-AMPS suelen ser duales para operar tanto a 800 en AMPS como a $1900 \mathrm{MHz}$ en PCS.

Cabe citar también el estándar cdmaOne, basado en el principio del CDMA (Code División Múltiple Access). En su evolución hacia la tercera generación, rebautizado como cdma2000, podrá ser utilizado en las redes UMTS.

\subsubsection{La tercera generación: UMTS.}

Las dos grandes características del mundo de las comunicaciones móviles son su rápido crecimiento y la enorme disparidad de sistemas, tecnologías y terminales que dificultan, cuando no impiden, la interoperatividad. La aparición de normas europeas como GSM, DECT (Digital Enhanced Cordless Telecommunications) y ERMES (European Radio Message System), permitió solventar en parte el problema, facilitando la circulación de los usuarios a nivel mundial [2.2].

Los principales requisitos de los sistemas celulares de tercera generación son :

$\infty$ Soportar servicios de baja y alta velocidad (voz, video, datos etc.) con altos niveles de calidad.

- Operar en un entorno multicelular (macro, micro y picocélulas).

- Operar en un entorno multioperador (redes públicas y privadas).

- Optimizar la utilización del espectro.

UMTS forma parte de la tercera generación de sistemas móviles. Deberá ofrecer, con una calidad muy superior a la de los actuales sistemas, un amplio rango de servicios, desde voz a transmisión de datos, imágenes y multimedia. Este estándar incluye servicios de baja y alta velocidad y la interoperación con las redes fijas de voz y datos.

UMTS representa una evolución en términos de servicios y velocidades de datos respecto a la segunda generación redes móviles. UMTS es la opción natural evolutiva para los operadores de GSM, con una clientela potencial de más de 747 millones de usuarios finales en más de 180 países, y una cuota de mercado de más del $70 \%$ [2.3].

Japón lanzó la primera red de WCDMA comercial en 2001, y en el último trimestre de 2002 
Hutchison abrió su propia red a ciertos clientes. UMTS se encuentra igualmente en estado precomercial en el Reino Unido, Italia, Suecia y otros territorios europeos.

La tendencia en los primeros terminales de usuario UMTS es la de aparatos multibanda y multimodo, que operan tanto en UMTS como en GSM, ofreciendo servicios de GPRS. Se pretende seguir con la tendencia a potenciar las comunicaciones de datos, cuya importancia no para de crecer. Así, se contabilizan actualmente más de 24 mil millones de mensajes de texto (SMS: Short Message Service) enviados cada mes, a los que hay que añadir ahora los mensajes multimedia (MMS: Multimedia Message Service). UMTS supondrá la transición hacia un futuro multimedia móvil.

\subsubsection{Características.}

\section{Interfaz de radio.}

Con la llegada de los sistemas móviles de tercera generación, se hace patente la necesidad de desarrollar nuevas tecnologías que permitan un máximo aprovechamiento del espectro. Además, la coexistencia de diferentes sistemas y la movilidad de los usuarios dificultan el diseño de terminales multibanda, puesto que en determinados casos las bandas de frecuencias se solapan. En la Tabla 2.3 se muestran las bandas de frecuencias reservadas para los principales sistemas de comunicaciones móviles celulares existentes hoy en día. Se aprecia cómo, por ejemplo las bandas de DCS y PCS coinciden, por lo que el ancho de banda de un equipo que opere en ambos sistemas aumenta considerablemente.

Tabla 2.3: Bandas de frecuencia de los sistemas de inalámbricos y celulares.

\begin{tabular}{|c|c|}
\hline Sistema & Bandas de frecuencia (MHz) \\
\hline AMPS/D-AMPS & $825-845 / 870-890$ \\
\hline PCS & $1850-1990 / 2180-2200$ \\
\hline E-GSM & $880-915 / 935-960$ \\
\hline DCS & $1710-1785 / 1805-1880$ \\
\hline UMTS FDD & $1920-1980 / 2110-2170$ \\
\hline UMTS TDD & $1900-1920 / 2010-2025$ \\
\hline
\end{tabular}

Aparte de los sistemas celulares, hay que tener en cuenta hoy en día la existencia de otros estándares, que a menudo coexisten con los anteriores en el mismo terminal. La Tabla 2.4 recoge las frecuencias destinadas a algunos de estos sistemas. Entre ellos cabe destacar aquellos en los que cualquier usuario puede operar sin licencia, como la banda ISM (Industrial, Scientific and Medical), utilizada para aplicaciones como control de sensores o apertura remota de puestas. También conviene resaltar el auge de los sistemas destinados a eliminar los cables para la 
interconexión de equipos, como los sistemas WLAN (Wireless Local Area Network) y, en menor medida, Bluetooth.

Tabla 2.4: Otras bandas de frecuencias.

\begin{tabular}{|c|c|}
\hline Sistema & Banda de frecuencias $(\mathbf{M H z})$ \\
\hline DECT & $1880-1900$ \\
\hline ISM & $2400-2500$ \\
\hline Bluetooth & $2400-2500$ \\
\hline WLAN & $2400-2500$ \\
\hline GPS & $1570-1580 / 1575,2$ \\
\hline DECT & $1880-1900$ \\
\hline ERMES & $169.4-169.8$ \\
\hline
\end{tabular}

\section{Evolución de la capacidad.}

El desarrollo de las comunicaciones móviles está íntimamente asociado con el de la transmisión de los datos en las redes móviles. Ahí reside de hecho la diferencia entre los sistemas de segunda generación, orientados hacia servicios de voz, y los de tercera generación, diseñados para datos [2.4]. En la Tabla 2.5 se muestra la evolución en la velocidad de transmisión de las redes existentes y futuras. La tendencia muestra que nos encontramos aún en una fase temprana de desarrollo en este campo. Lo mismo se podría decir de las aplicaciones WLAN y otros sistemas de radio.

La gráfica de la Figura 2.1 [2.4] muestra la evolución de la velocidad obtenida en función de la fecha de puesta en marcha de algunos sistemas móviles. Se observa que los diferentes puntos forman una línea recta. Teniendo en cuenta que la velocidad se representa de forma logarítmica, se tiene que dicha velocidad aumentaría de forma exponencial con el tiempo. De seguir esta tendencia hacia el año 2010 se podría disponer de sistemas con una capacidad de transmisión de $155 \mathrm{Mb} / \mathrm{s}$.

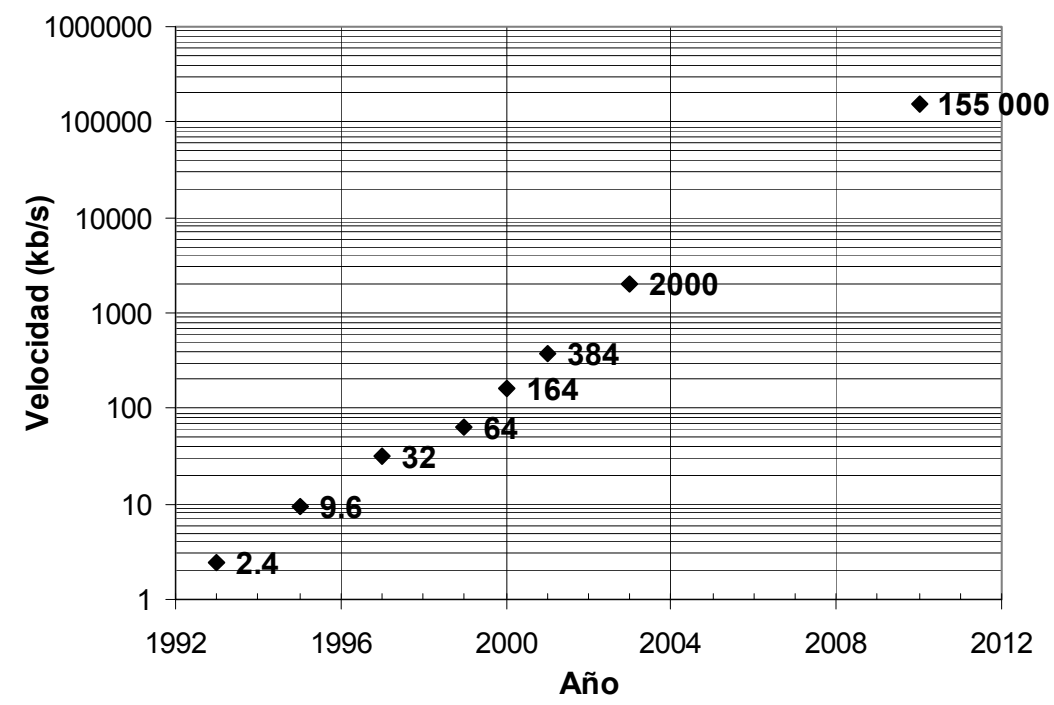

Figura 2.1: Evolución de la velocidad máxima de transmisión disponible. 
Tabla 2.5: Características de los sistemas celulares.

\begin{tabular}{|c|c|c|c|}
\hline Sistema & Fecha de inicio & Velocidad máxima (kb/s) & Banda de frecuencias $(\mathrm{GHz})$ \\
\hline PDC & 1993 & 2,4 & $0,8 / 1,5$ \\
\hline PDC & 1995 & 9,6 & $0,8 / 1,5$ \\
\hline GSM & 1995 & 9,6 & $0,9 / 1,8 / 1,9$ \\
\hline PDC-P & 1997 & 28,8 & 1,5 \\
\hline PHS & 1997 & 32 & 1,9 \\
\hline cdmaOne (IS-95A) & 1998 & 14,4 & $0,8 / 1,9$ \\
\hline D-AMPS (IS-136) & 1999 & 19,2 & $0,8 / 1,9$ \\
\hline PHS & 1999 & 64 & 1,9 \\
\hline GSM-HSCSD & 1999 & 64 & $0,9 / 1,8 / 1,9$ \\
\hline cdmaOne (IS-95B) & 2000 & 64 & $0,9 / 1,9$ \\
\hline PHS & 2000 & 128 & 1,9 \\
\hline GSM-GPRS & 2000 & 164 & $0,9 / 1,8 / 1,9$ \\
\hline cdmaOne (1XRTT) & 2001 & 144 & $0,8 / 1,9$ \\
\hline GSM-EDGE & 2001 & 384 & $0,9 / 1,8 / 1,9$ \\
\hline UMTS & 2003 & 2000 & 2.0 \\
\hline MBS & ¿? & 155000 & $40 / 60$ \\
\hline
\end{tabular}

\subsection{PRoblemática de diseño.}

\subsubsection{Terminales y funcionalidad.}

El tamaño de los terminales de comunicaciones móviles se ha reducido a pasos agigantados en los últimos años, al tiempo que aumentan sus funcionalidades. La Figura 2.2 ilustra perfectamente esta tendencia. De los voluminosos terminales de finales de los 80, que apenas permitían comunicaciones de voz, se ha pasado a microteléfonos con aplicaciones multimedia que caben en cualquier bolsillo. En el diseño de los terminales actuales dominan criterios como la resistencia mecánica, preferencias estéticas, la preocupación por el efecto sobre el usuario [2.5] y la necesidad de desarrollar antenas altamente eficientes, que optimicen el uso de la potencia disponible, alargando así la duración de las baterías [2.6]. Así, el mercado exige terminales pequeños, ligeros, y de bajo consumo, pero que tengan además un diseño atractivo y aplicaciones llamativas, como por ejemplo, tonos de llamada polifónicos, juegos, cámara integrada y aplicaciones multimedia. 


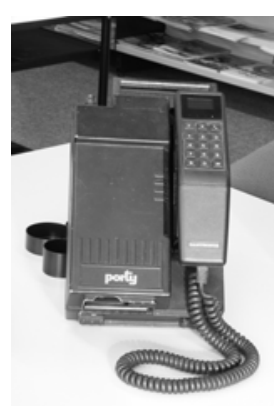

Finales de los 80

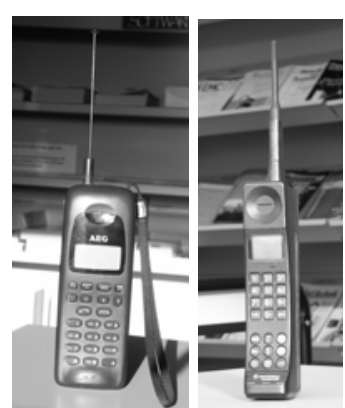

1992

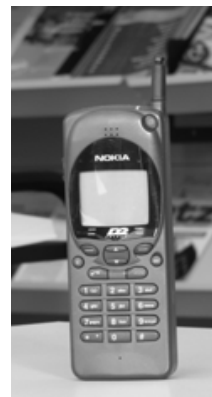

1995

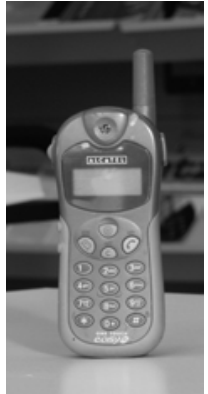

1997
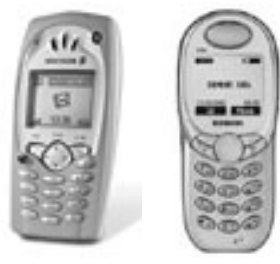

2002

Figura 2.2: Evolución de los terminales de comunicaciones móviles.

Algunos de los factores fundamentales que condicionan el diseño de antenas para terminales de comunicaciones móviles son:

- La necesidad de antenas eléctricamente pequeñas o con formas determinadas, limitadas a un pequeño volumen. Esto da lugar a restricciones de polarización, pérdida de eficiencia de radiación y de ancho de banda, mientras que aumenta la sensibilidad a las tolerancias de fabricación.

\& Un plano de masa limitado a la carcasa del terminal, por lo que tiene dimensiones del mismo orden de magnitud que la longitud de onda a la frecuencia de trabajo. Esto afecta a las características de adaptación, el ancho de banda, el diagrama de radiación y la interacción con el usuario.

\& Una adaptación a la entrada de $50 \Omega$, para obtener buenos valores de eficiencia en transmisión y una buena relación señal-a-ruido en recepción.

- Un ancho de banda suficiente para cubrir los estándares considerados. El ancho de banda está determinado generalmente por la impedancia de entrada a la frecuencia de operación, pero también por la eficiencia y el diagrama de radiación.

\& En caso de antenas multibanda, mecanismos que permitan ajustar independientemente cada una de las bandas de frecuencias consideradas.

- Buenas características de radiación, incluso en presencia del usuario. En su mano y cabeza se producirán una pérdida de potencia debida a fenómenos de absorción.

$\infty$ Niveles de SAR (Specific Absorption Rate) dentro de los límites permitidos, con tendencia reducirlos lo máximo posible.

Por otra parte, los fabricantes requieren que las antenas que van a emplear sean de bajo coste, fácil fabricación y fácil montaje, idealmente tipo SMD (Surface Mounted Devices), para su producción en serie. En terminales cuyo precio de mercado ronda los $300 €$, la antena debe costar menos de $0,5 €$.

Cada vez más, los fabricantes se decantan por el uso de antenas integradas, que permiten cumplir con estos requisitos. Entre ellas, las antenas tipo parche son las más extendidas, y serán las que se considerarán en este trabajo. Con ellas se obtienen resultados óptimos en lo referente a eficiencia, 
relación calidad/precio, y niveles de SAR.

Hay que tener en cuenta, además, que los terminales actuales son, casi en su totalidad, equipos multiestándar. Así, en el mercado se encuentran terminales capaces de operar en dos o más bandas de frecuencias. La tendencia es utilizar para ello una única antena, con varios modos resonantes. Esto es posible usando parches multiresonantes.

Se ha considerado en algún momento la posibilidad de reutilizar la misma antena interna en diferentes terminales [2.7]. Para ello habría que lograr concentrar las corrientes en un entorno cercano al parche, con lo que se logra reducir el efecto del usuario, pero se pierde igualmente ancho de banda. Sin embargo, un diseño individualizado parece más apropiado, debido a la influencia que tiene toda la estructura del terminal sobre el comportamiento final en radiación. Así, hay que considerar siempre múltiples factores como las dimensiones finitas del plano de masa [2.8], su tamaño total [2.9], o la posición relativa de la antena en el terminal [2.10].

\subsubsection{Efecto del plano de masa finito.}

Las antenas son generalmente medidas y analizadas en un entorno electromagnético bien definido, con planos de masa infinitos o grandes con respecto a la longitud de onda de trabajo. Sin embargo, en el caso de terminales móviles de comunicaciones la antena debe funcionar sobre una carcasa eléctricamente pequeña, que distorsiona sus características de funcionamiento. Así, una antena no puede ser considerada como un elemento aislado, sino que forma parte de un sistema mayor. Es importante determinar cómo el hecho de integrar una antena en un terminal afectará a su funcionamiento real, en lo concerniente tanto a adaptación de impedancias como a sus características de radiación [2.11].

Los campos generados por la antena inducen corrientes que fluyen por los distintos componentes del terminal, especialmente el PCB (Printed Circuit Board o pletina). Se ha demostrado que los efectos más apreciables de la integración de una antena en una caja metálica son una desintonización de la frecuencia de operación, cierta desviación del diagrama de radiación y un incremento de las componentes de polarización cruzada, debido a las corrientes inducidas en la superficie del terminal [2.8], [2.12].

\subsubsection{Restricciones en el tamaño y forma de la antena.}

Como ya se ha mencionado anteriormente, el tamaño de los terminales móviles disminuye rápidamente. Los progresos en el campo de la microelectrónica permiten obtener cada vez circuitos más compactos, y así reducir las dimensiones y el peso de los equipos. Sin embargo, en lo referente a la antena, las limitaciones de tamaño no están determinadas por factores tecnológicos, sino físicos. Para que se produzca una radiación la estructura debe tener un determinado tamaño, 
adaptado a la longitud de onda de la frecuencia de trabajo.

En antenas instaladas en equipos móviles los requisitos de pequeño tamaño, bajo peso y volumen reducido adquieren una gran importancia. Esto convierte a las antenas impresas en una interesante alternativa a las antenas tradicionales. Sin embargo, a las frecuencias de operación de los sistemas de comunicaciones móviles, el parche convencional en $\lambda_{\mathrm{g}} / 2$ resulta ser demasiado grande. Por ello hay que buscar alternativas que permitan reducir el tamaño total sin que por ello sus características de funcionamiento se vean degradadas.

\subsection{FACTOR HUMANO.}

Un hecho importante que se debe tener en cuenta es que, en condiciones normales de funcionamiento, el móvil será utilizado por un operador humano. La presencia del usuario debe ser considerada no sólo en términos de la potencia absorbida por en el cuerpo humano, reflejada en de Tasa de Absorción Específica (Specific Absorption Rate, SAR) [2.13], [2.14]. Debido a la proximidad del tejido humano, se producirá además una modificación de la distribución de los campos electromagnéticos, que afectará al rendimiento del terminal. El usuario representa pues una fuente de distorsión para la señal emitida [2.15].

\subsubsection{Efecto sobre el usuario (SAR).}

Como los terminales móviles operan muy cerca del usuario, merece especial atención la interacción de los campos electromagnéticos radiados con el tejido humano. Para las bandas de frecuencias utilizadas en comunicaciones móviles, el efecto dominante es la absorción de energía electromagnética por los tejidos [2.16]. Debido a esta interacción electromagnética, una cierta cantidad de energía radiada por la antena del terminal es absorbida por el cuerpo del usuario, principalmente la mano y la cabeza.

La potencia absorbida por el tejido humano viene dada por la siguiente fórmula:

$$
\mathrm{P}_{\mathrm{abs}}=\frac{1}{2} \int_{\mathrm{V}} \sigma|\mathrm{E}|^{2} \mathrm{dV}
$$

Donde E representa el valor de pico del campo eléctrico, $\sigma$ la conductividad del medio, en este caso los tejidos, y $\mathrm{V}$ el volumen de tejido considerado. $\mathrm{P}_{\mathrm{abs}}$ refleja el porcentaje de potencia que absorbe el usuario respecto a la potencia entregada a la antena.

Esta absorción se produce de forma local, y su distribución es inhomogénea. Su efecto se traduce en un aumento local de la temperatura de los tejidos, que se puede expresar en términos de la Tasa 
de Absorción Específica, o SAR (Specific Absorption Rate). La SAR, expresada en $\mathrm{mW} / \mathrm{g} / \mathrm{W}$, refleja la velocidad a la que el tejido humano absorbe la energía de microondas por unidad de peso y de potencia de transmisión.

$$
\mathrm{SAR}=\mathrm{c} \frac{\mathrm{dT}}{\mathrm{dt}}
$$

En la expresión (2.2), c representa el calor específico, y $\frac{\mathrm{dT}}{\mathrm{dt}}$ el incremento de la temperatura en los tejidos en función del tiempo. Esta expresión se puede escribir también en función de los parámetros del material y del valor del campo eléctrico como:

$$
\operatorname{SAR}=\frac{\sigma}{2 \rho}|\mathrm{E}|^{2}=\frac{\sigma}{\rho} \mathrm{E}_{\text {eff }}^{2}
$$

Donde $\rho$ denota la densidad del material, $\sigma$ su conductividad y $E_{\text {eff }}$ el valor efectivo del campo eléctrico. Esta última dependerá de la frecuencia de trabajo considerada.

Los valores que se utilizan para la caracterización del tejido humano en las frecuencias de GSM y DCS se recogen en la Tabla 2.6 [2.17].

Tabla 2.6: Valores utilizados para las simulaciones de tejido humano.

\begin{tabular}{|c|c|c|c|}
\hline & $\varepsilon_{\mathbf{r}}$ & $\sigma(\mathbf{S} / \mathbf{m})$ & $\boldsymbol{\rho}(\mathbf{k g} / \mathbf{m} 3)$ \\
\hline $900 \mathrm{MHz}$ & 42,5 & 0,86 & \multirow{2}{*}{1040} \\
\cline { 1 - 3 } $1800 \mathrm{MHz}$ & 41 & 1,69 & \\
\hline
\end{tabular}

Los límites de exposición son diferentes según la región y el estándar considerados. En Europa las medidas se rigen por la recomendación 1999/519/EC, de acuerdo con CENELEC, que toma los valores dados por la ICNIRP [2.16]. En Estados Unidos, el organismo regulador FCC establece que las medidas han de ajustarse a la norma ANSI C95.1 [2.18]. En ambos casos no se toma el máximo local absoluto, sino que se realiza un promediado sobre un volumen cúbico de masa determinada. Los límites de SAR local establecidos, así como los valores utilizados para el promediado, se recogen en la Tabla 2.7 .

Tabla 2.7: Límites de SAR local en la cabeza.

\begin{tabular}{|c|c|c|}
\hline & $\begin{array}{c}\text { SAR máxima } \\
\text { local (W/kg) }\end{array}$ & $\begin{array}{c}\text { Masa para el } \\
\text { promediado (g) }\end{array}$ \\
\hline Europa & 2 & 10 \\
\hline Estados Unidos & 1,6 & 1 \\
\hline
\end{tabular}

En la Figura 2.3 se muestra el sistema de medida DASY (Dosimetric Assessment SYstem), utilizado para las medidas de SAR. Un brazo articulado permite colocar la sonda de medida dentro de la cubeta que contiene el líquido equivalente al tejido humano. 


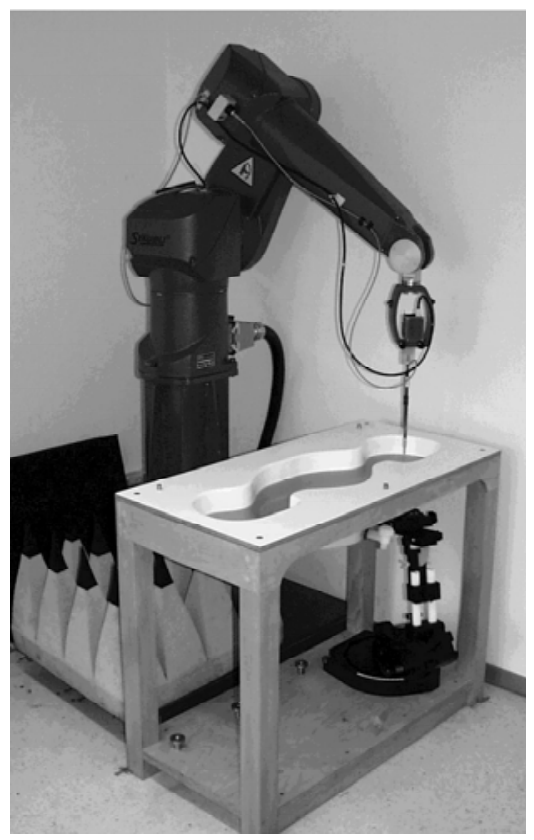

Figura 2.3: Sistema de medida DASY.

\subsubsection{Efecto del usuario.}

Desde el punto de vista de la eficiencia de la antena, y del terminal en su conjunto, la presencia del usuario supone un aumento drástico de las pérdidas que hay que tener en cuenta. También hay que considerar que los tejidos humanos presentan valores muy elevados de permitividad, lo que puede producir fuertes modificaciones en la adaptación de la antena. Finalmente, los diagramas de radiación se verán también afectados.

Así, la potencia radiada en la zona de campo lejano, para el caso de un terminal en condiciones normales de funcionamiento, se puede escribir:

$$
\mathrm{P}_{\mathrm{rad}}=\mathrm{P}_{\text {in }}-\mathrm{P}_{\mathrm{a}}-\mathrm{P}_{\mathrm{L}}-\mathrm{P}_{\mathrm{abs}}
$$

Donde $\mathrm{P}_{\text {rad }}$ representa la potencia realmente radiada en campo lejano, $\mathrm{P}_{\text {in }}$ la potencia entregada, $\mathrm{P}_{\mathrm{a}}$ las pérdidas por la desadaptación a la entrada de la antena, incluyendo las debidas al usuario, $\mathrm{P}_{\mathrm{L}}$ las pérdidas en la propia antena y $\mathrm{P}_{\mathrm{abs}}$ la potencia absorbida por el usuario.

En [2.19] se identifican algunas causas de discrepancia entre los resultados obtenidos para diferentes usuarios, en cuanto a pérdidas por absorción en el cuerpo. Entre ellas se pueden destacar:

y Posición de la mano sobre el teléfono.

$\infty$ Distancia entre la cabeza y el teléfono.

\& Ángulo de inclinación del teléfono.

$\infty$ La forma de la cabeza y la mano.

$\infty$ El tamaño de la persona.

- Otros parámetros: edad, sexo, presencia de empastes en la boca, uso de gafas... 
Las dos partes del cuerpo que más influyen en el funcionamiento del móvil son, por su proximidad, la cabeza y la mano del usuario. En el caso de la cabeza, su efecto puede ser medido con relativa facilidad, utilizando el modelo (phantom) relleno de solución salina de la Figura 2.4.

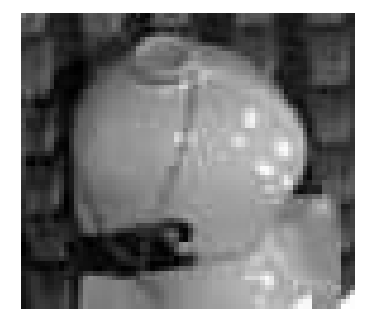

Figura 2.4: Modelo de cabeza utilizado en las medidas.

El efecto de la mano es más difícil de cuantificar, puesto que cada usuario sujeta el terminal de forma distinta. Este problema es especialmente importante en el caso de antenas internas, ya que en determinados casos se puede llegar a enmascarar totalmente la antena y degradar de forma significativa el enlace. En [2.19] se describe la correlación entre la forma en que el usuario sujeta el terminal y las pérdidas, y se llega a la conclusión de que, en la mayoría de los casos, dominan las pérdidas por absorción en la mano frente a las causadas por la posición relativa entre el terminal en la cabeza.

El efecto del usuario sobre los diagramas de radiación se puede determinar utilizando el modelo de la Figura 2.4, y el dispositivo de medidas 3D mostrado en la Figura 2.5 [2.20].

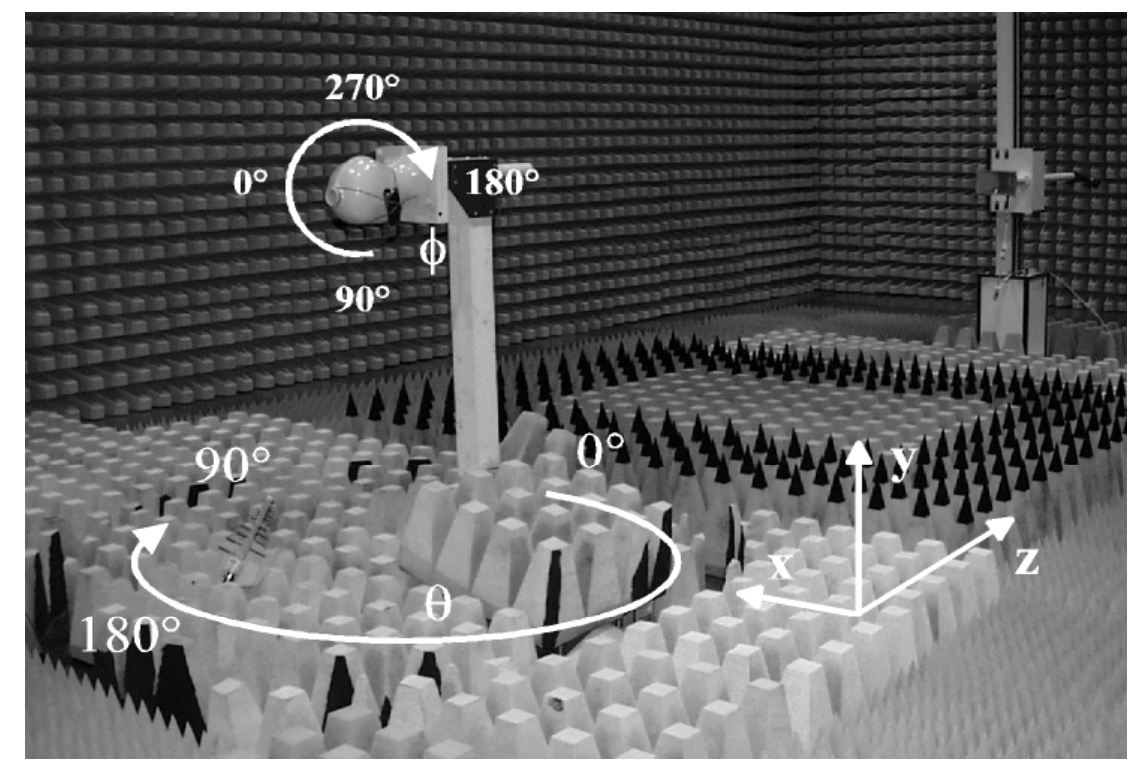

Figura 2.5: Dispositivo de medidas 3D del diagrama de radiación incluyendo el efecto del usuario.

\subsubsection{Efecto del entorno.}

El entorno de operación de los terminales móviles impone ciertos requisitos que han de tenerse en cuenta a la hora de diseñar antenas para este tipo de aplicaciones. El suelo, los edificios e incluso la 
proximidad del usuario son factores que contribuyen al aumento de interferencias y ruido térmico. Además, el usuario ha de poder mantener conversaciones sin interrupciones en multitud de entornos y situaciones diferentes.

Otra de las variables introducidas por el usuario es la orientación espacial del terminal. Hoy en día no se puede describir una situación típica de utilización, por cuanto las nuevas funciones integradas en los terminales móviles hacen imposible predecir si éste estará en contacto con el cuerpo, situado sobre una superficie conductora, si se utiliza un equipo "manos libres"...

Además, el terminal puede estar situado cerca de todo tipo de superficies, o en la proximidad de otros equipos, como por ejemplo un ordenador portátil. Así, el problema es especialmente importante en el caso de antenas para Bluetooth o WLAN, o antenas integradas en terminales tipo "palmtop", o tarjetas PCMCIA.

\subsection{ANTENAS EXTERNAS.}

\subsubsection{Monopolos.}

El monopolo fue el primer elemento considerado para los terminales móviles. Durante años fue la antena utilizada por la casi totalidad de los fabricantes, debido a su gran ancho de banda, a su diagrama de radiación omnidireccional y a su bajo coste. La geometría de un monopolo en $\lambda_{\mathrm{g}} / 4$ montado sobre un terminal se muestra en la Figura 2.6.
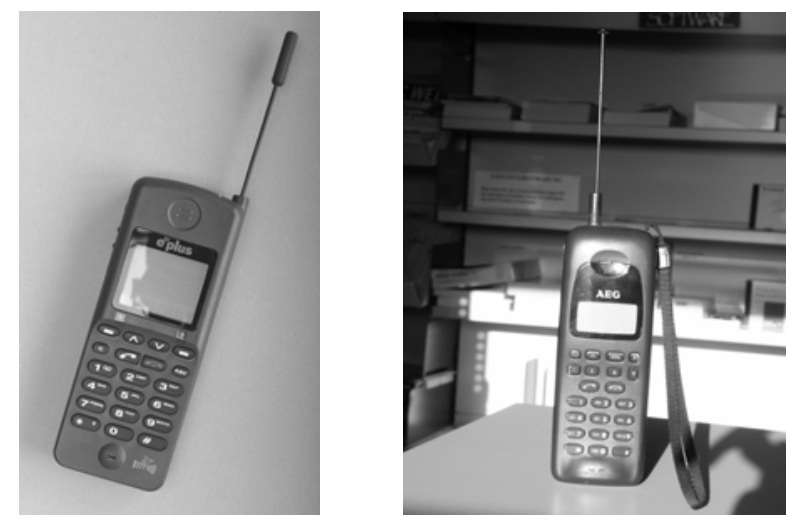

Figura 2.6: Terminal con monopolo en $\lambda / 4$.

Este tipo de antenas presenta sin embargo algunas desventajas, ligadas a su geometría. En primer lugar, su longitud, de unos $8 \mathrm{~cm}$ para terminales que operan en la banda de GSM, es demasiado grande para los terminales actuales. Además, este elemento resulta poco resistente a los impactos. Para mejorar su resistencia, se puede recurrir a elementos retráctiles, como el mostrado en la Figura 2.7. Es el usuario el que debe extender la antena al utilizar el terminal, lo que puede resultar molesto. 


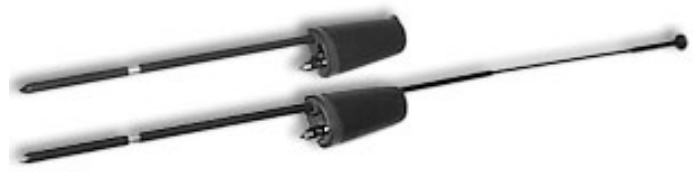

Figura 2.7: Monopolo retráctil.

Otra desventaja del monopolo reside en los niveles de SAR que genera, muy cercanos a los límites establecidos. Debido a todos estos factores, el monopolo ha sido eliminado casi en su totalidad del mercado de los teléfonos móviles, aunque subsiste en ciertas áreas como Norteamérica, o para ciertos terminales tipo "Palmtop".

\subsubsection{Hélices.}

El uso de las hélices radiantes, como las mostradas en la Figura 2.8, se impuso para hacer frente a los problemas planteados por los monopolos. Con ello se logra que los terminales tengan un aspecto más compacto, y sean más resistentes a los golpes. Además, ya no resulta necesario extraer la antena con cada utilización del aparato. Algunos ejemplos de terminales con antenas de hélice se recogen en la Figura 2.9.
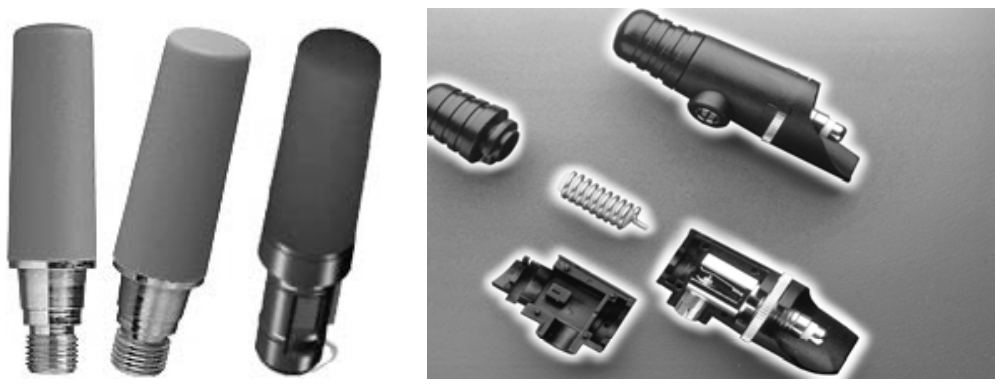

Figura 2.8: Antenas de hélice.
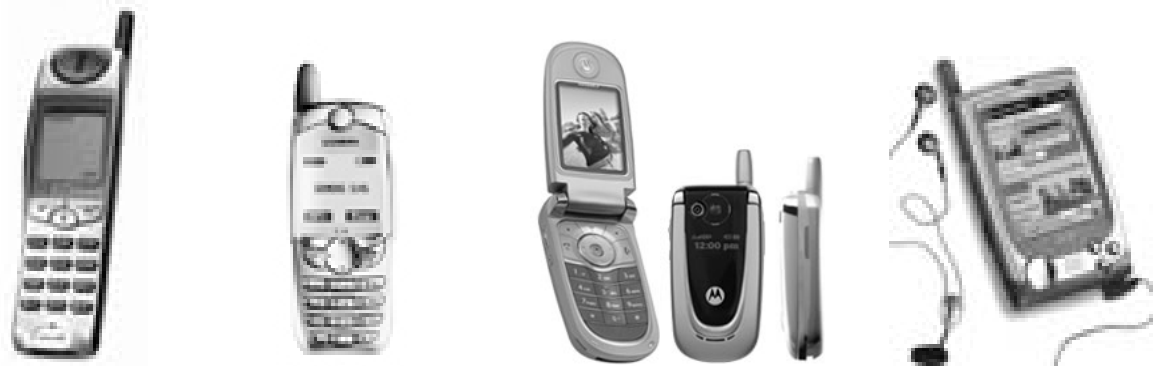

Figura 2.9: Terminales comerciales con antenas de hélice.

Parte del éxito de las hélices es debido a que con ellas resulta relativamente sencillo desarrollar elementos multibanda. Una manera de hacerlo es usando diferentes hélices, o combinando la hélice con un monopolo, tal y como se muestra en la Figura 2.10 para el caso de antenas de doble banda [2.21]. En la Figura 2.11 se recoge un ejemplo de terminal con este tipo de antenas para modo de operación dual. 


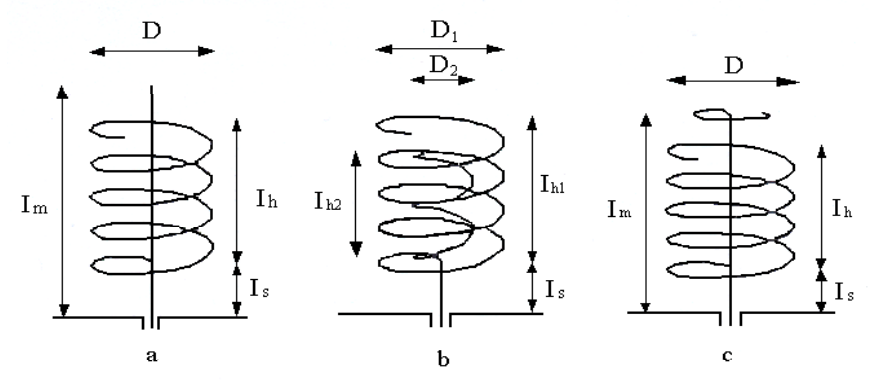

Figura 2.10: Combinaciones para obtener hélices duales.

(a) Combinación hélice-monopolo.

(b) Dos hélices concéntricas.

(c) Combinación hélice-monopolo cargado.

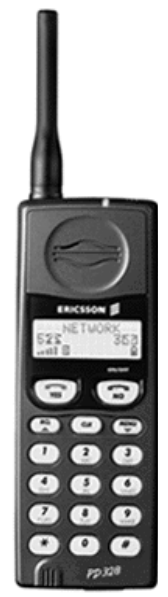

Figura 2.11: Terminal dual con combinación de hélice y monopolo.

El uso de varios elementos combinados resulta sin embargo costoso en cuanto a precio y complejidad de fabricación, por lo que no suele ser muy utilizado. En la práctica, se desarrollan antenas multibanda ajustando el paso y el diámetro de la hélice para conseguir múltiples resonancias [2.22].

Un caso particular de la antena en hélice es la línea con meandros (meander line antenna), presentada en la Figura 2.12. Se puede considerar como una proyección plana de la hélice convencional. Consiste en una línea metálica impresa sobre un soporte dieléctrico, normalmente flexible. Esta línea actúa como un monopolo, pero su longitud se acorta doblándola un determinado número de veces.

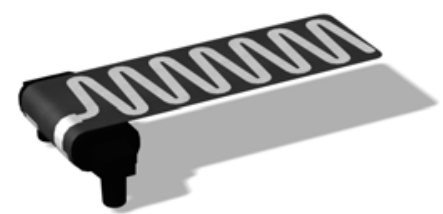

Figura 2.12: Antena “meander line”.

También es ente caso se pueden ajustar las frecuencias de resonancia y la impedancia de entrada variando el número de meandros o su ancho. Utilizando más de una línea, y/o ajustando su ancho y 
la distancia entre meandros se puede lograr también un modo de operación multibanda [2.23], [2.24].

Este tipo de antenas se puede encontrar en numerosos terminales, e incluso en tarjetas PCMCIA, tal y como se muestra en la Figura 2.13. En algunos casos puede ser utilizada también como antena interna, aunque su rendimiento disminuye entonces considerablemente.
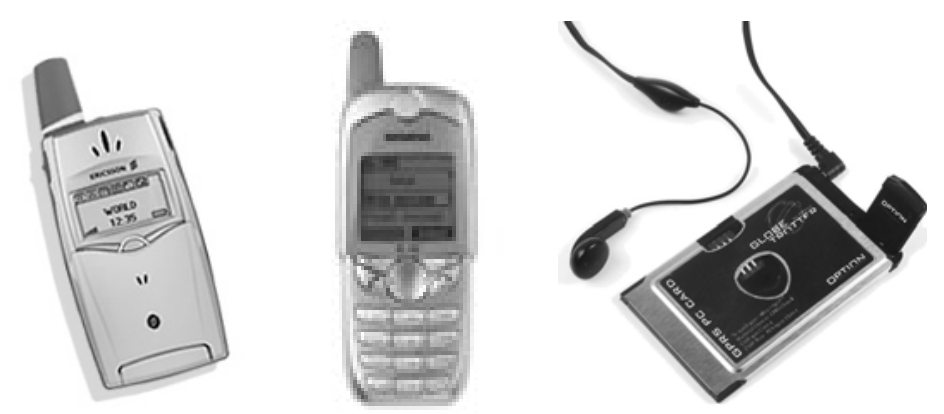

Figura 2.13: Ejemplos de equipos con antena "meander line".

\subsection{ANTENAS INTERNAS.}

\subsubsection{Consideraciones de diseño.}

Dados los requisitos impuestos en los sistemas de comunicaciones móviles, las antenas internas se han convertido en una alternativa altamente atractiva. Cada vez más los usuarios se decantan por terminales más ligeros y compactos, que se puedan llevar cómodamente en un bolso o un bolsillo. Así aparecen en el mercado nuevos terminales, más ligeros y pequeños, con bajo consumo de energía y diseño atractivo.

El uso de antenas internas responde también a determinadas estrategias de márketing. Con ellas se concede una mayor libertad a los diseñadores, ya que no tienen que tener en cuenta ningún elemento externo a la hora de concebir nuevos aparatos. También supone un aumento de la robustez del terminal, por cuanto la antena está más protegida frente a golpes, y el usuario ya no tiene acceso directo a ella. En la Figura 2.14 se recogen algunos ejemplos de terminales con antena interna.
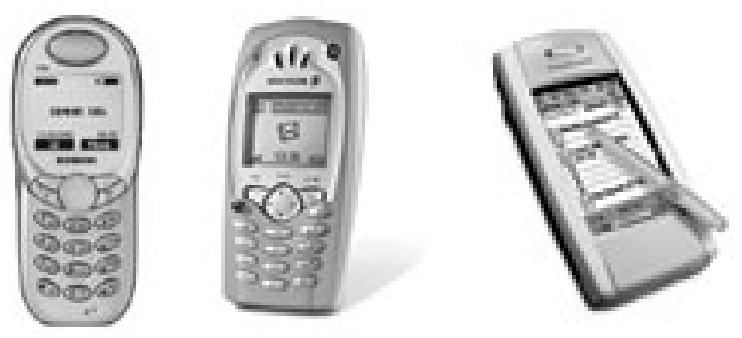

Figura 2.14: Ejemplos de terminales con antena integrada. 
Los fabricantes apuestan también por este tipo de antenas debido a su facilidad de fabricación y de montaje, y a los bajos costes que se obtienen para grandes volúmenes de producción. En la Figura 2.15 se describe esquemáticamente el problema: dentro del terminal, se dispone de un determinado volumen en del cual se debe integrar la antena. Este volumen suele ser pequeño en términos de longitud de onda, por lo que se debe optimizar el uso del espacio disponible, y aplicar técnicas de miniaturización.

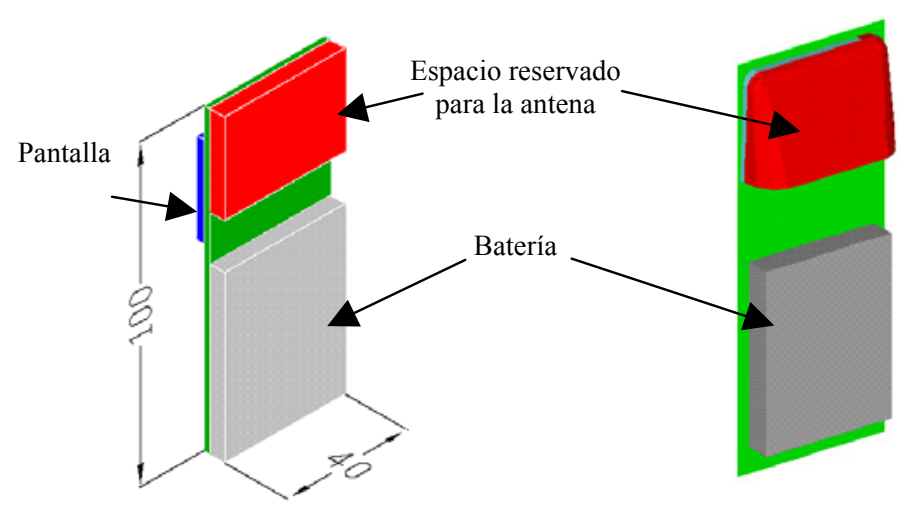

Figura 2.15: Descripción del problema típico.

El diseño de este tipo de antenas supone un verdadero reto, puesto que los factores que influyen en su funcionamiento son tan numerosos que resulta imposible considerarlos en su totalidad.

Hay que tener en cuenta además que los terminales actuales tienen que operar normalmente en dos o más bandas de frecuencias, mientras que el volumen disponible para la antena es cada vez más reducido. Debido a ello, los requerimientos de diseño se han flexibilizado con respecto a los que se establecían hace algunos años en cuanto a pérdidas de retorno y forma del diagrama de radiación.

\subsubsection{Tecnologías consideradas.}

En lo referente a antenas integradas para terminales de comunicaciones móviles, existen tres alternativas principales: los parches metálicos, las antenas 3D-MID y las antenas con substrato cerámico. Sus principales características, así como ventajas e inconvenientes, se detallan a continuación.

\section{Parches metálicos.}

Las antenas parche son, hoy en día, las más comunes en los terminales de comunicaciones móviles, debido a su flexibilidad, bajo coste y facilidad de fabricación.. Consisten en una simple lámina metálica que sirve como elemento radiante. Dos ejemplos de las mismas se muestran en la Figura 2.16. El parche puede ser plano, o bien adaptarse a la forma de la cubierta del terminal, aunque dentro de ciertos límites. En efecto, la estructura metálica y el proceso de fabricación por estampado limitan los grados de libertad en lo referente a pliegues y curvatura. 


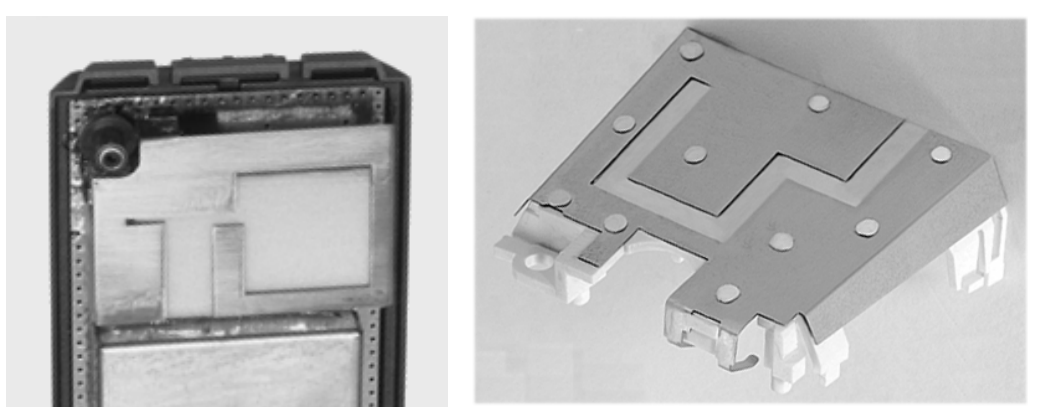

Figura 2.16: Antenas parche.

El problema que se presenta con el uso de parches radiantes es su fijación al terminal. En la Figura 2.17 se recogen las soluciones más corrientes. La primera sería fijar la antena sobre un soporte de plástico, que a su vez se sujeta a la pletina del terminal. Con ello se consigue una estructura muy estable, resistente a las tolerancias de fabricación. También se puede estampar directamente la antena sobre la cubierta posterior del aparato, con lo que se puede ahorrar peso y costes. Se pierde sin embargo en robustez frente a las tolerancias, y la conexión con la líneas de alimentación es menos fiable.

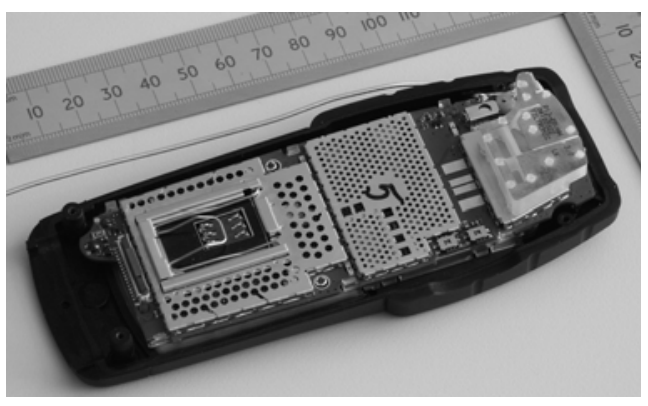

(a)

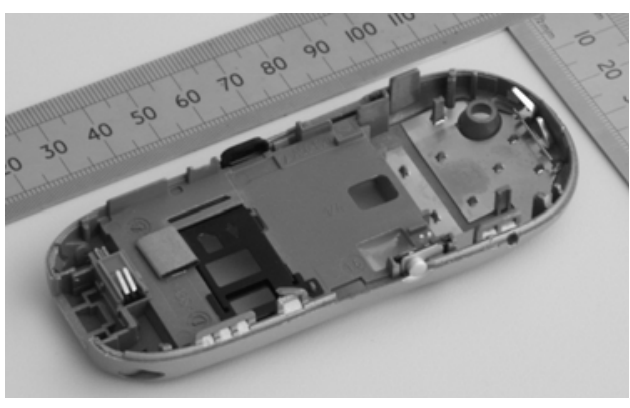

(b)

Figura 2.17: Terminales con antena integrada sobre soporte de plástico (a) o fijada a la cubierta posterior (b).

Una variante de las antenas parche consiste en utilizar estructuras metálicas impresas sobre materiales flexibles (Flexlayer) como los utilizados para las líneas de transmisión de circuitos impresos [2.25]. Se alcanza así una gran flexibilidad en el diseño de la estructura de la antena, aunque los pliegues del material siguen estando limitados a dos dimensiones. También es necesario resolver el problema de la fijación de la estructura, para lo que se pueden utilizar por ejemplo, soportes de plástico como el de la Figura 2.18. La capa flexible puede ser fijada por medios mecánicos, utilizando pegamento o por medios térmicos.

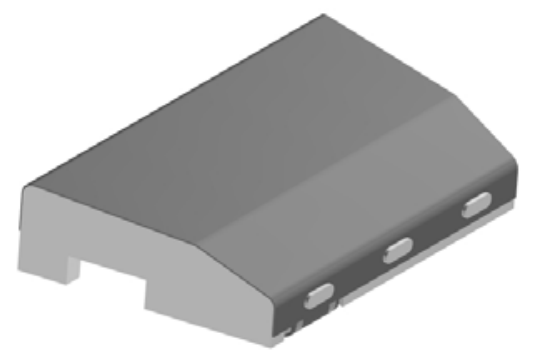

Figura 2.18: Antena impresa en substrato flexible sobre soporte de plástico. 


\section{2. $3 D-M I D$.}

Una alternativa interesante es la utilización de tecnología 3D-MID (Metal Injected Devices). Con la deposición por medios químicos de metal sobre un cuerpo de plástico se pueden obtener estructuras de forma totalmente tridimensional, con lo que se sobrepasan las limitaciones de pliegues $2 \mathrm{D}$ de los parches convencionales. Así, el aspecto de una antena 3D-MID genérica sería el mostrado en la Figura 2.19. El objetivo en este caso es optimizar el uso del volumen disponible para la realización de la antena.

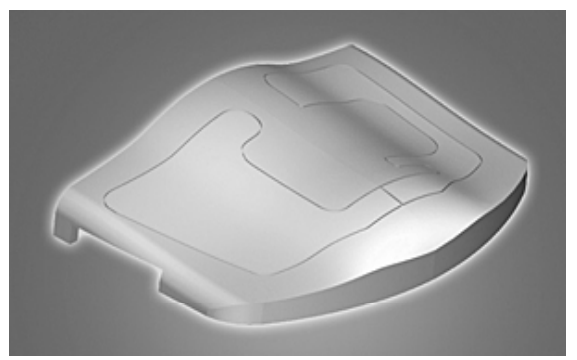

Figura 2.19: Estructura típica de una antena 3D-MID.

Esta tecnología fue la utilizada en la realización de la primera antena integrada en un terminal de telefonía móvil. En la Figura 2.20 se puede apreciar que en aquel caso no existían demasiadas restricciones en lo referente al volumen de la antena, diseñada para operar en la banda de GSM.
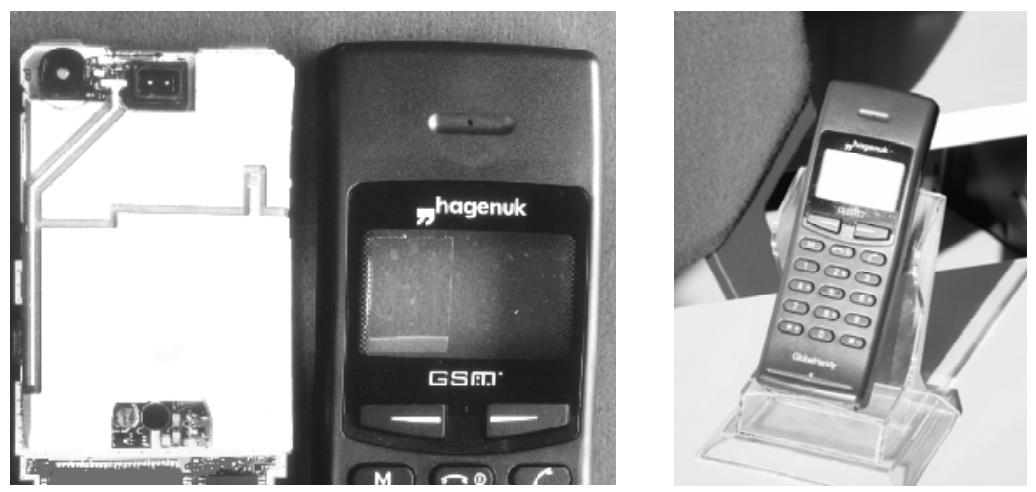

Figura 2.20: Primer móvil con antena integrada.

Unos terminales más modernos, en este caso de doble banda GSM/DCS, se recogen en la Figura 2.21. Se observa cómo el soporte metálico puede seguir la forma de la cubierta del terminal, aprovechándose así al máximo el espacio disponible.
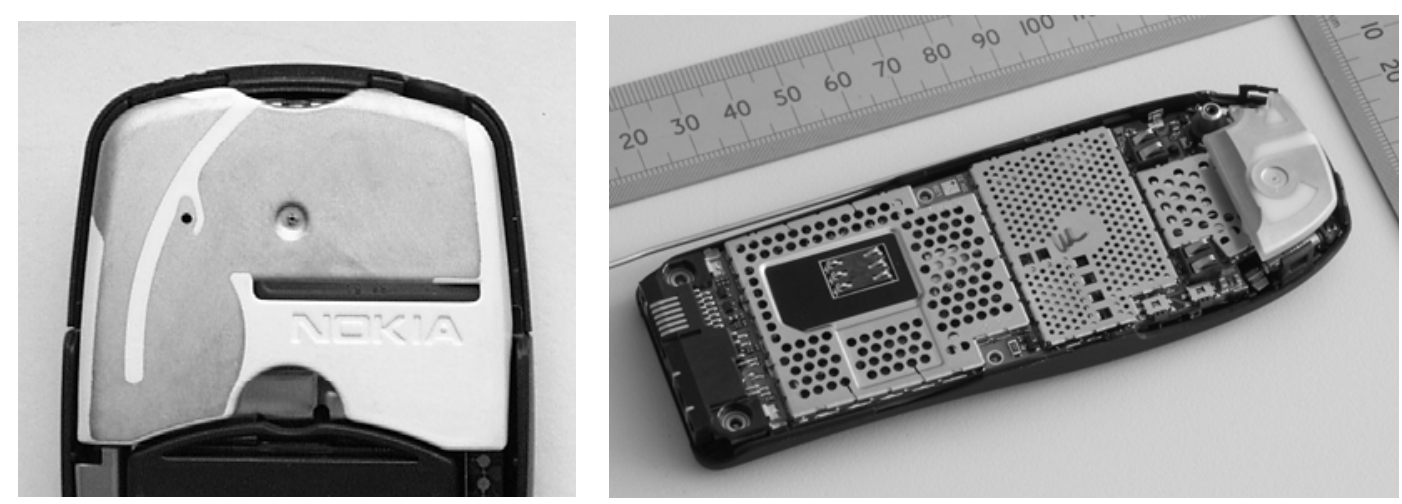

Figura 2.21: Terminales con antena integrada 3D-MID. 
La tecnología 3D-MID presenta, sin embargo, importantes inconvenientes. Por una parte, los materiales utilizados normalmente, tanto dieléctricos como metales, tienen mayores pérdidas que en el caso de las antenas parche convencionales, por lo que su eficiencia es menor. También resulta más complicado y costoso realizar cambios en la estructura, ya que es necesario entonces fabricar nuevos moldes y cambiar la maquinaria. Por lo tanto, los costes de fabricación son mucho más elevados, y este tipo de antenas sólo resulta rentable para grandes volúmenes de producción.

\section{Antenas sobre substrato cerámico.}

Una opción para miniaturizar las antenas es el uso de substratos dieléctricos de alta permitividad. En eso se basa el uso de substratos cerámicos para la fabricación de antenas para móviles, como las mostradas en la Figura 2.22.

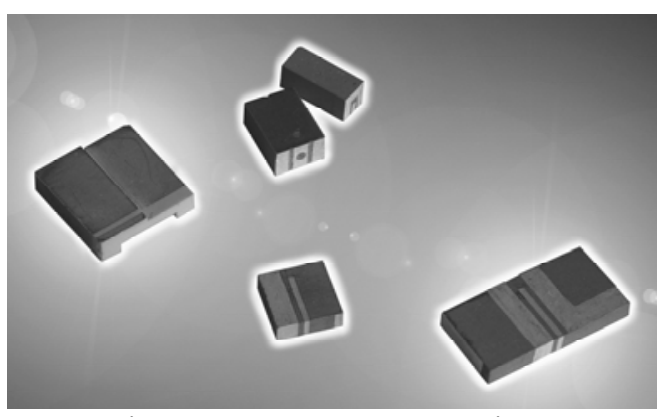

Figura 2.22: Antenas cerámicas.

Estas antenas han sido utilizadas con éxito para aplicaciones de comunicaciones inalámbricas, pero su utilización en los terminales de telefonía móvil plantea grandes problemas. Por una parte, al usar un material de alta permitividad, se reduce la eficiencia y el ancho de banda de la antena. Son además estructuras muy sensibles a los materiales conductores situados en su proximidad. Se tiene que eliminar a menudo la metalización de la pletina en el área de la antena, lo que provoca normalmente un aumento de los valores de la SAR.

Otros inconvenientes de estas antenas son su peso, elevado en relación con el peso total del terminal, y su coste y su dificultad de fabricación.

Una alternativa sería la utilización de finas láminas de cerámica sobre las que se imprime la estructura metálica de la antena, utilizando tecnología LTCC (Low Temperature Co-fired Ceramics). El substrato LTCC consiste en un apilamiento de láminas cerámicas que se usan para aplicar pastas conductivas, dieléctricas y/o resistivas. Así es posible producir circuitos multicapa sobre el mismo substrato. Las láminas juntan y cuecen en un solo paso a temperaturas relativamente bajas, $\left(850^{\circ} \mathrm{C}-875^{\circ} \mathrm{C}\right)$, por lo que es posible usar conductores poco resistivos como la plata y el oro.

La ventaja de esta tecnología es que se pueden integrar en el mismo substrato tanto la antena como, por ejemplo, los circuitos de radiofrecuencia. Esto se ha realizado ya con éxito en el caso del módulo Bluetooth presentado en la Figura 2.23 [2.26]. 


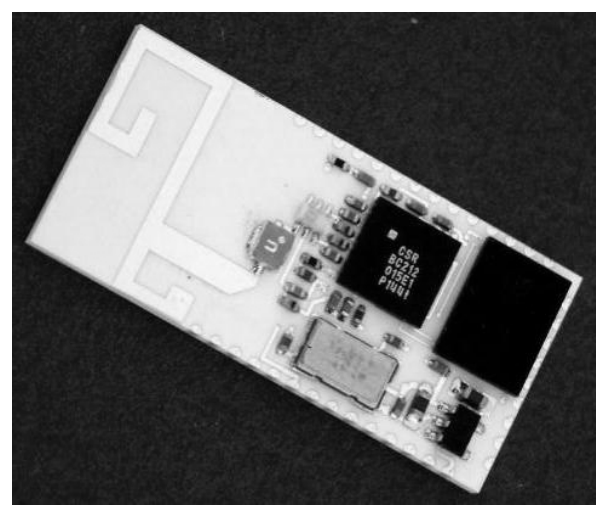

Figura 2.23: Módulo Bluetooth sobre LTCC con antena integrada.

Esta tecnología se encuentra todavía, en lo que a antenas se refiere, en fase experimental. Su coste es relativamente elevado para pequeñas cantidades, y la estructura final resulta frágil, por lo que su integración en terminales móviles debe ser aún resuelta.

\subsection{Conclusión.}

En este capítulo se ha descrito brevemente el estado de la técnica en cuanto a estándares de comunicaciones móviles, problemática de diseño en lo referente a terminales, y antenas para móviles. En los capítulos posteriores se considerarán únicamente las antenas de tipo parche, cuya versatilidad permite satisfacer los requerimientos establecidos. Para su análisis se recurrirá a los conceptos de base relacionados con las antenas impresas.

\subsection{Biblografía.}

[2.1] Mouly, M. y Pautet, M.B., 'Current Evolution of the GSM Systems', IEEE Personal Communications, 1995, vol. 2, nº5, pp. 9-19.

[2.2] Schwarz-Dasilva, J., Ikonomou, D. y Erben, H., 'European R\&D Programs on Third Generation Mobile Communications Systems', IEEE Personal Communications, 1997, vol. $4, n^{\circ} 2$, pp. $46-47$.

[2.3] UMTS Forum, http://www.umts-forum.org.

[2.4] Correia, L.M., en 'Wireless Flexible Personalised Communications, COST 259: European Co-operation in Mobile Radio Research', editado por L.M. Correia, John Wiley \& Sons, 2001.

[2.5] Mittra, R. y Dey, S., 'Challenges in PCS Antenna Design', IEEE Antennas and Propag. 
Society Symp., 1999, pp. 544-547.

[2.6] Pedersen, G.F. y Bach Andersen, J., 'Integrated Antennas for Hand-Held Telephones with Low Absorption', IEEE Vehicular Technol. Conf., 1994, pp. 1537-1540.

[2.7] Garner, W.J. y Lu, W.L.: 'Designing Antennas for Cellular Telephones', Microwaves \& $R F, 1999$, vol. $38, \mathrm{n}^{\circ} 5$, pp. 70-76.

[2.8] Sato, K., Matsumoto, K., Fujimoto, K. y Hirasawa, K., 'Characteristics of a Planar Inverted-F Antenna on a Rectangular Conducting Body', Electronics and Communications in Japan, 1989, vol. 72, $\mathrm{n}^{\circ}$ 10, pp. 43-51.

[2.9] Luebbers, R., Chen, L., Uno, T. y Adachi, S., 'FDTD Calculation of Radiation Patterns, Impedance, and Gain for a Monopole Antenna on a Conducting Box', IEEE Trans. on Antennas and Propagat., 1992, vol. 40, n 12, pp. 1577-1583.

[2.10] Geissler, M., Heberling D. y Wolf, I.: 'Properties of Integrated Handset Antennas', Millenium Conf. on Antennas and Propagat.. AP2000, 2000, p. 637.

[2.11] Yamaguchi, R., Sawaya, K., Fujino Y. y Adachi, S., 'Effect of Dimension of Conducting Box on Radiation Pattern of a Monopole Antenna for Portable Telephone', IEEE Antennas and Propagat. Society Symp., 1992, pp. 669-672.

[2.12] Taga, T. y Tsunekawa, K., 'Performance Analysis of a Built-In Planar Inverted-F Antenna for $800 \mathrm{MHz}$ Band Portable Radio Units', IEEE Journal on Selected Areas on Communications, 1987, vol. 5, nº5, pp. 921-929.

[2.13] Ghandi, O.P., Lazzi, G. y Furse, C.M., 'Electromagnetic Absorption in the Human Head and Neck for Mobile Telephones at 835 and 1900 MHz', IEEE Transactions on Microwave Theory and Tech., 1996, vol. 44, n 10, pp. 1884-1897.

[2.14] Ehmann, R., Burkhardt, T. Wolter, H. y Weiland, T., 'Electromagnetic Simulation of a Loaded Cavity’, IEEE Transactions on Magnetics, 1996, vol. 32, nº 3, pp. 922-925.

[2.15] Jansen, M. y Rahmat-Samii, Y., 'EM Interaction of Handset Antennas and a Human in Personal Communications', Proceedings of the IEEE, vol. 83, $\mathrm{n}^{\circ}$ 1, Enero 1995, pp. 7-17.

[2.16] ICNIRP, 'Guidelines for Limiting Exposure to Time-Varying Electric, Magnetic and Electromagnetic Fields (up to $300 \mathrm{GHz}$ ), Health Physics, 1998, vol. 74, pp. 449-522.

[2.17] CENELEC, European Specification ES 59005, 'Considerations for the Evaluation of Human Exposure to Electromagnetic Fields (EMFs) from MobileTelecommunication Equipment (MTE) in the Frequency Range from $30 \mathrm{MHz}-6 \mathrm{GHz}$, Ref. n ES 59005:1998 E, 1998.

[2.18] ANSI C95.1, 'IEEE Standard for Safety Levels with Respect to Human Exposure to Radio Frequency Electromagnetic Fields, 3KHz to $300 \mathrm{GHz}$ ', 1991.

[2.19] Pedersen, G.F., 'Antennas for Mobile Phones', en 'Wireless Flexible Personalised 
Communications, COST 259: European Co-operation in Mobile Radio Research', editado por L.M. Correia, John Wiley \& Sons, 2001.

[2.20] Bornkessel, C., Heberling, D., Neikes, M. y Schramm, A., '3D Radiated Power Meaurements of Phones at IMST with Regard to User Interaction', COST 260 Meeting and Workshop on Smart Antennas, Goteborg, 2001.

[2.21] Erätuuli, P., Haapala, P. y Vainikainen, P., 'Dual Frequency Wire Antennas', Electronics Letters, 1996, vol. 32, nº 12, pp. 1051-1052.

[2.22] Ying, Z., 'Multiband Non-Uniform Helical Antennas', Patente US6054966, Estados Unidos, 2000.

[2.23] Teng, P.L. y Wong, K.L., 'Planar Monopole Folded into a Compact Structrure for VeryLow-Profile Multi-Band Mobile Phone Antenna', 2002, Microwave and Optical Tech. Lett., vol. 33, $\mathrm{n}^{\circ} 1$, pp. 22-25.

[2.24] Lee, G.Y. y Wong, K.L., 'Very-Low-Profile Bent Planar Monopole Antena for GSM/DCS Dual-Band Mobile Phone', Microwave and Optical Tech. Lett., 2002, vol. 34, n 6, pp. 406-409.

[2.25] Nicholson, M., 'Flexible Printed Antennas', Microwaves \& RF, Noviembre 2002, pp. 6468.

[2.26] Suárez-Pejenaute, E., Baggen, R. y Martínez-Vázquez, M., 'Integrated Antennas on LTCC Substrate for Bluetooth Applications', Journées Internationales de Nice sur les Antennes, 2002. 



\section{Capítulo 3: INTRODUCCIÓN A LAS ANTENAS IMPRESAS.}

Las ciencias aplicadas no existen, sólo las aplicaciones de la ciencia.

Louis Pasteur

\subsection{INTRODUCCIÓN.}

Las antenas impresas han sido en las últimas décadas el tema central de numerosos proyectos, relacionados mayoritariamente con arrays para aplicaciones de satélites o en sistemas de comunicaciones móviles. Mucho se ha avanzado desde que Deschamps [3.1] propusiera por primera vez el concepto de antena impresa en 1953, aunque la autoría puede ser también atribuida a otros autores, como Greig y Engleman [3.2] y Lewin [3.3]. Hubo que esperar sin embargo hasta los años 70 para que se le prestara la suficiente atención al concepto, y para que se publicaran las primeras ecuaciones de diseño.

Hoy en día existen una serie de libros que conviene usar como base para cualquier trabajo que tenga que ver con las antenas impresas, como los publicados por Bahl y Barthia [3.4], James, Hall y Wood [3.5] y James y Hall [3.6]. Algunos de ellos son relativamente antiguos y, salvo en el caso de aquellos que han sido reeditados en los últimos años, no recogen los últimos desarrollos en este campo tan dinámico. Sin embargo siguen siendo elementos de referencia, por cuanto permiten comprender el funcionamiento básico de estas estructuras y proporcionan la necesaria metodología de diseño.

Otras referencias más actuales, que complementan los contenidos de las anteriores serían los libros editados por Hirasawa y Haneishi [3.7], y Lee y Chen [3.8].

En estos momentos se puede considerar que la tecnología de las antenas impresas ha alcanzado su madurez. Ahora que su funcionamiento se conoce casi por completo, sólo queda explotar sus casi infinitas posibilidades. En este caso, su aplicación a los terminales de comunicaciones móviles. 


\subsection{CARACTERÍSTICAS DE LAS ANTENAS IMPRESAS.}

Una antena impresa consiste básicamente en un parche situado sobre un plano de masa y separado de éste por una fina capa de material dieléctrico, denominado substrato, tal y como se muestra en la Figura 3.1. Generalmente las dimensiones de la antena son del orden de una fracción de la longitud de onda a la frecuencia de trabajo. La forma del parche impreso es arbitraria, aunque los más comunes, y por consiguiente los que han sido estudiados más en detalle, son los parches cuadrados, rectangulares, romboidales, triangulares, bicónicos, circulares, elípticos o, en general, con formas geométricas regulares [3.4]. La forma del parche se elige pues según las necesidades, permitiendo así satisfacer diferentes especificaciones relativas, por ejemplo, al ancho de banda, polarización o diagrama de radiación.

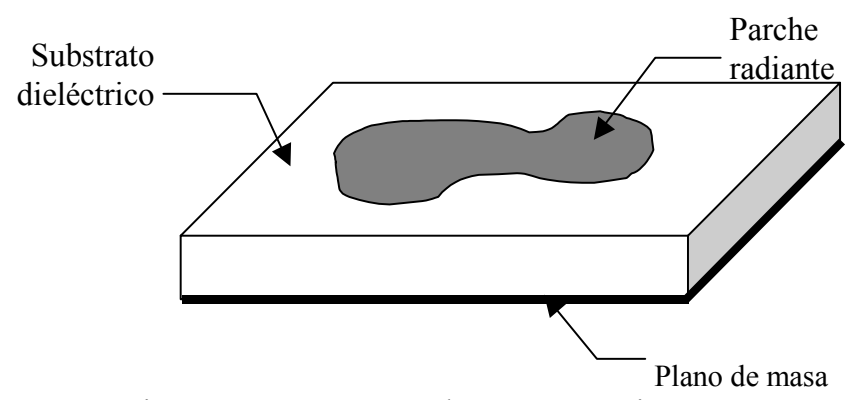

Figura 3.1: Estructura de una antena impresa.

La permitividad del substrato suele ser relativamente baja (entre 2 y 10), aunque en determinados casos, y para cumplir con los requisitos establecidos, se pueden encontrar substratos con permitividades mayores. Si se requieren bajas pérdidas y una alta eficiencia de radiación se utilizan substratos de permitividad cercana a 1, como espumas, o simplemente aire. Sin embargo, si lo que importa es reducir el tamaño de la antena, se debe recurrir a substratos de mayor permitividad, a costa de perder ancho de banda.

Existen distintas formas de excitar antenas impresas. Dos de ellas, por sonda coaxial y por línea microtira, se muestran en la Figura 3.2.
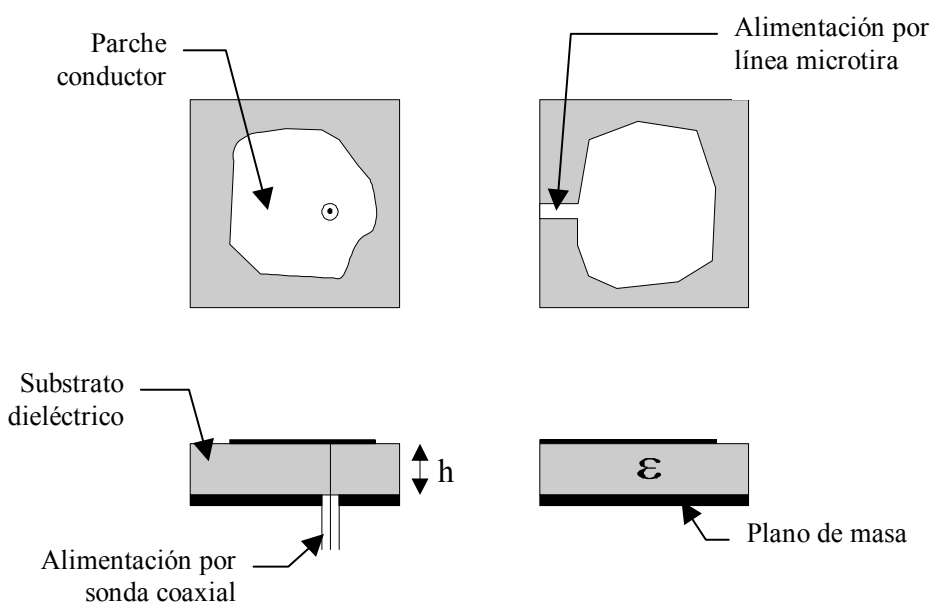

sonda coaxia
(a) Alimentación coaxial
(b) Alimentación por línea microtira.
Figura 3.2: Alimentación de una antena impresa. 
La línea de alimentación guía la energía electromagnética hasta la región situada bajo el parche. La energía se distribuye por esta región, y parte de ella es radiada hacia el exterior. Éste es el origen de uno de los problemas de las antenas impresas: la región bajo el parche es, en sí, una cavidad resonante con un alto factor de calidad Q. Por lo tanto la eficiencia de radiación y los anchos de banda que se pueden obtener son limitados.

El margen de frecuencias en que se suelen emplear las antenas impresas es muy amplio: desde 100 $\mathrm{MHz}$ hasta $50 \mathrm{GHz}$. Con respecto a las antenas de microondas convencionales, presentan ciertas ventajas como:

- Bajo coste de fabricación

- Características físicas: bajo peso, pequeño volumen, perfil plano

\&ácil diseño de elementos multifrecuencia

$\infty$ Control de la polarización mediante la posición del punto de alimentación

$\infty$ Estructura conformada

- Compatibilidad con la tecnología de circuitos integrados

$\infty$ Posibilidad de integrar en la estructura las líneas de alimentación y las redes de adaptación

$\infty$ Facilidad de formación de arrays

$\infty$ Facilidad de integración

Sin embargo presentan también una serie de inconvenientes:

$\infty$ Baja eficiencia

Escaso ancho de banda

- Límite práctico de la ganancia (unos $20 \mathrm{~dB}$ )

$\infty$ Efecto de las ondas de superficie

$\infty$ Complejidad de la red de alimentación

$\infty$ Si el plano de masa es de grandes dimensiones, la radiación se produce en un solo hemisferio

- Dificultad para obtener gran pureza de polarización

- Radiaciones espurias (alimentación, ondas de superficie)

$\infty$ Requieren substratos de alta calidad, capaces de soportar grandes cambios de temperatura.

Algunos de estos inconvenientes se pueden solventar, sacrificando algún otro parámetro y alcanzando compromisos de diseño adaptados a la aplicación considerada, tal y como se recoge en la Tabla 3.1 para el caso de parches rectangulares.

Aunque sólo determinadas formas regulares han sido estudiadas en detalle, con ecuaciones que permiten predecir sus características, Pichon [3.9] propuso una técnica para determinar la impedancia de entrada de antenas impresas de forma arbitraria. Para ello utilizaba una combinación del método MPIE (Mixed Potential Integral Equation) con el Método de los Momentos (MoM), 
dividiendo el parche en celdas triangulares y superponiendo las funciones base en pares de celdas. La corriente de excitación se propaga radialmente alrededor de una celda triangular por superposición de tres funciones de base solapadas que tienen como vértice común el centro de la celda triangular. Esta técnica ha sido aplicada con éxito a parches rectangulares y triangulares.

Tabla 3.1: Compromisos de diseño de un parche rectangular.

\begin{tabular}{|c|c|c|c|}
\hline Requisito & $\begin{array}{c}\text { Grosor h del } \\
\text { substrato }\end{array}$ & $\begin{array}{c}\text { Permitividad del } \\
\text { substrato }\end{array}$ & $\begin{array}{c}\text { Ancho W del } \\
\text { parche }\end{array}$ \\
\hline Alta eficiencia de radiación & grueso & baja & ancho \\
\hline Bajas pérdidas dieléctricas & delgado & baja & - \\
\hline Bajas pérdidas en conductoras & grueso & - & - \\
\hline Gran ancho de banda & grueso & baja & ancho \\
\hline Baja radiación espuria & delgado & baja & - \\
\hline Baja polarización cruzada & - & baja & - \\
\hline Bajo peso & delgado & baja & - \\
\hline Robustez & grueso & alta & ancho \\
\hline $\begin{array}{c}\text { Baja sensibilidad a las } \\
\text { tolerancias }\end{array}$ & grueso & baja & \\
\hline
\end{tabular}

\subsection{ANTENA IMPRESA RECTANGULAR.}

La estructura más simple que se puede considerar es la antena impresa rectangular. Consiste en un parche rectangular de longitud L y ancho W, como el representado en la Figura 3.3. La longitud L corresponde a una fracción de la longitud de onda a la frecuencia de trabajo, mientras que el ancho se ajusta para satisfacer los requisitos de diseño. Esta configuración es una de las más extendidas, no sólo por su sencillez, sino también porque existen distintos modelos analíticos que describen acertadamente sus características de funcionamiento.

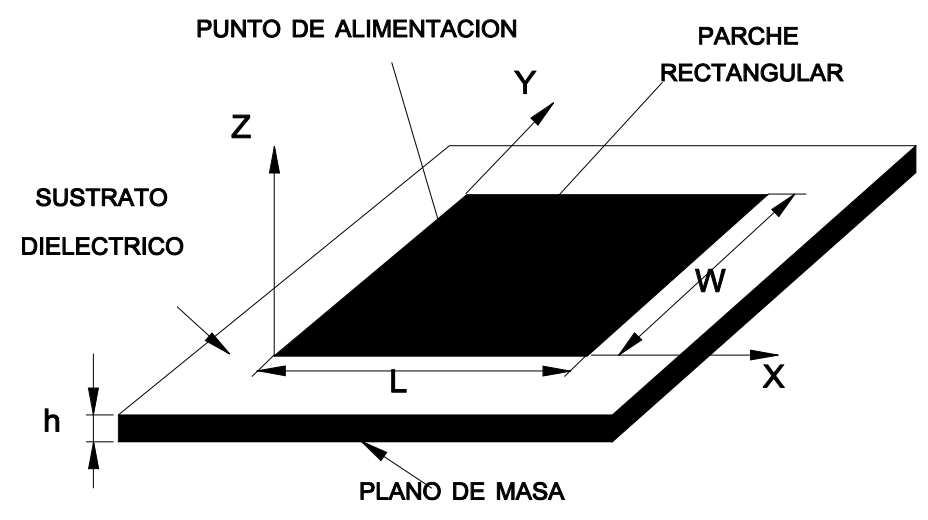

Figura 3.3: Antena impresa rectangular. 
Debido a estas razones, utilizaremos el ejemplo del parche rectangular para explicar el funcionamiento de las antenas impresas, así como sus principales características.

En el caso de que el parche radiante esté alimentado por una línea microtira la adaptación de impedancias resulta complicada, puesto que la impedancia de entrada de la antena puede alcanzar valores comprendidos entre cien y trescientos ohmios. Una red de adaptación puede llegar a ser necesario, especialmente si se trabaja con altas frecuencias.

En el caso de utilizar una línea microtira para la alimentación del parche existen dos procedimientos para lograr una buena adaptación y una impedancia de entrada conveniente:

- Cambiando la relación de aspecto de la antena, es decir, el cociente entre la longitud de los lados radiantes y no radiantes

- Realizando unas muescas para llevar la alimentación hasta un punto de la superficie de la antena en que la impedancia sea menor. Sin embargo resulta complicado situar con precisión ese punto, dándose discrepancias entre los resultados medidos y los obtenidos por simulación.

Para determinar la impedancia de entrada de antenas impresas rectangulares es común recurrir al Método de los Momentos de Galerkin. De hecho, Sipus et al. [3.10] proporcionan una expresión para la impedancia de entrada de antenas impresas rectangulares alimentadas por líneas microtira, teniendo en cuenta la contribución de modos de orden superior. Para ello utilizan como modelo de la línea de alimentación un hilo de corriente constante vertical entre el plano de masa en el punto en que se une la línea de alimentación y el parche. Calculan así el factor k, que tiene en cuenta el efecto del borde, y determinan la impedancia de entrada aplicando el método de los momentos. Se obtiene así la siguiente expresión:

$$
\begin{aligned}
& Z_{\text {in }}=-V_{1} I_{1}+j X_{f} \quad V_{1}=k \int_{0}^{k} E_{1} J_{i} d l \\
& k=\sqrt{\frac{W_{e}}{h}} \quad W_{e}=W_{f}+0,412 h\left(\frac{\varepsilon_{\text {eff }}+0,3}{\varepsilon_{\text {eff }}-0,258}\right)\left(\frac{W_{f}+0.262 h}{W_{f}+0.813 h}\right)
\end{aligned}
$$

En ella, $E_{1}$ designa el campo eléctrico del modo de expansión, $\mathrm{J}_{\mathrm{i}}$ es la densidad de corriente en el parche, $\mathrm{W}_{\mathrm{f}}$ el ancho de la línea de alimentación y h el espesor del substrato.

En el caso de alimentación por sonda coaxial, el conector interno se conecta al parche, mientras el externo se conecta al plano de masa. La energía electromagnética es así guiada hasta la región situada bajo el parche. Un modelo muy exacto de este tipo de alimentación es el propuesto por Hall [3.11]. Este modelo incluye un modo de conexión especial para tener en cuenta la discontinuidad existente en el punto de conexión entre el coaxial y el parche. Este modo de conexión es tratado como una función de base especializada dentro del Método de los Momentos, y se completa con una corriente vertical linealmente creciente a lo largo del conector, para reforzar la continuidad en el punto de conexión. 
Aboud [3.12] presentó asimismo un modelo simple para determinar la impedancia de entrada de un parche rectangular alimentado por sonda coaxial, adaptado a las necesidades de CAD (diseño asistido por ordenador). Este modelo se basa en el modelo de la cavidad para determinar la frecuencia de resonancia y la resistencia de entrada a dicha frecuencia, en la permitividad dinámica $\varepsilon_{\mathrm{dyn}}$ de un parche rectangular para tener en cuenta los efectos de borde, y en el circuito RLC resonante paralelo equivalente de la antena, que incluye una reactancia inductiva.

En [3.11] se recurría al Método de los Momentos para resolver numéricamente una ecuación de potencial mixto (MPE, Mixed Potential Equation) y calcular así los parámetros de interés de antenas con substratos gruesos. Anteriormente Gao [3.13] investigó el efecto del substrato en la distribución de corriente de una sonda coaxial, considerando un parche circular como un resonador con pérdidas con una determinada admitancia en las paredes (wall admitance). El método de Galerkin permitía entonces resolver la ecuación integral y obtener una expresión para la distribución de corriente en la sonda. A diferencia de los resultados obtenidos mediante métodos como la función de Green o la transformada de Hankel vectorial, que consideran una distribución de corriente uniforme para cualquier espesor de substrato, Gao establece que esto se cumple únicamente para substratos de espesor inferior a 0,3 veces la longitud de onda, esto es: $\mathrm{h} / \lambda_{\mathrm{g}}<0,3$.

Así pues, se han publicado estudios que incluían diferentes parámetros que influían en la impedancia de entrada las antenas impresas rectangulares. Entre los efectos considerados cabe destacar los debidos al espesor del substrato, y los producidos por las variaciones axiales y acimutales de la distribución de corriente en la superficie de la sonda coaxial.

Por otra parte, hay que tener en cuenta la posibilidad de que el parche se encuentre cubierto, de forma voluntaria o accidental, por una capa de dieléctrico. En ese caso, la frecuencia de resonancia se reduce, provocando problemas de desintonización y degradando seriamente las características de la antena [3.14]. Se pueden encontrar referencias que incluyen fórmulas de CAD para determinar la frecuencia de resonancia de antenas rectangulares con cubiertas dieléctricas [3.15].

Una alternativa bastante extendida para alimentar antenas impresas es el acoplamiento por apertura, presentado por Pozar [3.16]. En la Figura 3.4 se muestra cómo la antena impresa en un substrato dieléctrico está acoplada, a través de un plano metálico en el que se ha recortado una apertura, a una línea microtira situada en otro substrato paralelo al primero. Con este método se logra alimentar la antena sin necesidad de una conexión directa entre el parche y la línea de alimentación. Pozar demostró la validez de esta técnica con diferentes prototipos en la banda C (4-8 GHz) y la banda K (18-27 GHz). Desarrolló asimismo una teoría simplificada para el acoplamiento de la antena impresa a la línea microtira a través de una apertura, basándose para ello en el modelo de cavidad para el parche y en la teoría de la apertura de Bethe (Bethe hole theory) para acoplamiento por pequeñas aperturas. Según Pozar, la apertura debe tener el menor tamaño posible, mientras que la adaptación a la entrada se realizaría ajustando la longitud de la línea, que se comportaría como un stub en circuito abierto. 


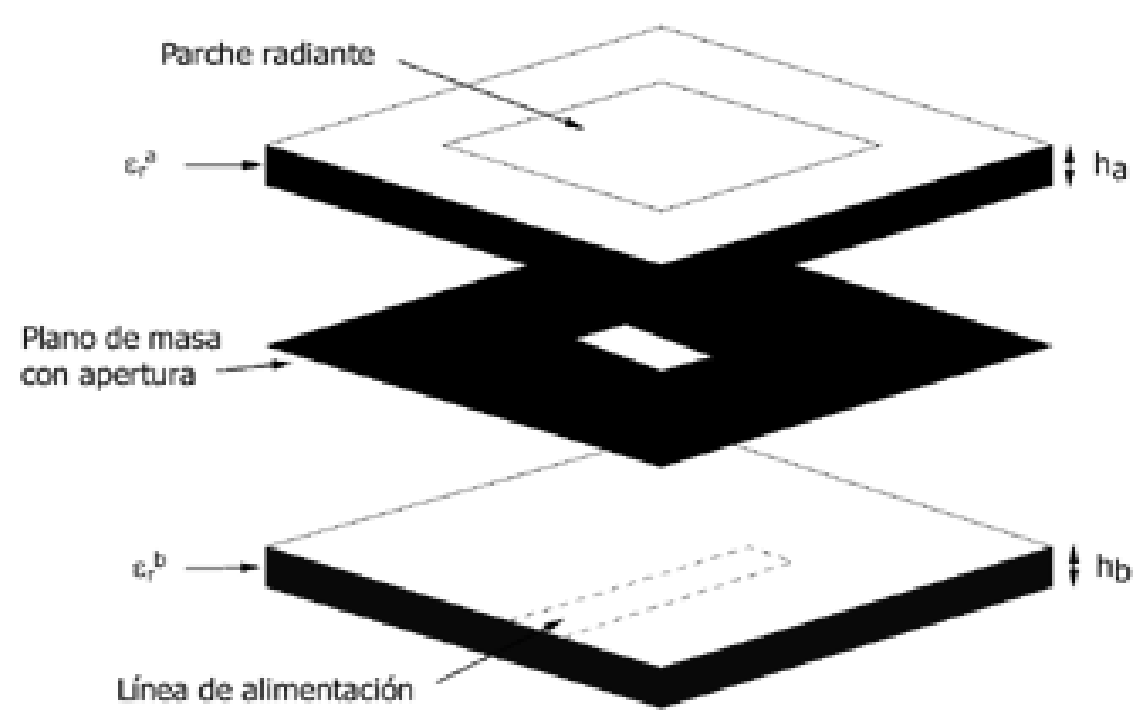

Figura 3.4: Antena alimentada por acoplamiento de apertura.

Existen análisis más detallados de este tipo de alimentación, entre los que se pueden citar los de Gao [3.17] y Sullivan [3.18]. Este último se sirve de las funciones de Green para dieléctricos sobre plano de masa para incluir en el análisis todos los efectos de acoplamiento, radiación y ondas de superficie que se originan en los dos substratos. Proporciona además curvas de diseño para determinar:

- el tamaño de la apertura, para ajustar la parte resistiva de la impedancia de entrada,

$\infty$ la longitud del stub en circuito abierto, para alcanzar la reactancia necesaria.

Aunque la impedancia de entrada del parche es relativamente insensible a pequeñas variaciones de la posición del parche sobre la apertura, sí varía de forma importante para desplazamientos mayores, con substratos tanto de alta como de baja permitividad. Sullivan [3.18] incluye además en su estudio un análisis de los parámetros $\mathrm{S}$, que resulta útil para el diseño de arrays alimentados en serie.

Gao, por su parte, obtiene la matriz de dispersión utilizando una apertura circular y una red equivalente de seis puertos. Este análisis resulta difícil utilizando las mismas técnicas que Sullivan, e incluye el efecto del espesor del plano de masa y de las variaciones de los campos en la apertura. Si el tamaño de la apertura es un punto crítico del diseño para obtener una buena adaptación en el rango de frecuencias de interés, la impedancia de entrada es al contrario bastante insensible frente a variaciones de espesor del plano de masa.

Este método presenta una serie de ventajas, ya que la red de alimentación está separada del elemento radiante por el plano de masa, lo cual previene la radiación espuria. Además las redes de acoplo y otros dispositivos pueden ser fabricados en un sustrato con una alta constante dieléctrica. Se pueden incluir así elementos activos en el mismo soporte, mientras que los elementos radiantes se situarían en otro substrato de menor permitividad para mejorar la eficiencia de radiación. 
Se han estudiado igualmente variaciones respecto a la forma original de la apertura. Pozar [3.20] propuso una nueva en forma de "hueso", consistente en la apertura original con dos ranuras en los extremos. Rostand [3.21] utilizó una alternativa de banda ancha para un array de recepción de televisión por satélite.

Otro método para alimentar antenas impresas consiste en una línea microtira con un hueco de discontinuidad (gap) bajo el parche, tal y como propuso Chung [3.22]. El acoplamiento entre el gap de la línea de alimentación y el parche se puede controlar mediante el offset. Éste método puede resultar útil para la alimentación de arrays en serie.

Se ha considerado también la posibilidad de utilizar fibras ópticas coplanares en diferentes casos, tal como se muestra en [3.23].

\subsubsection{Mecanismo de radiación.}

La radiación de las antenas impresas se debe a los campos electromagnéticos existentes entre los bordes del parche y el plano de masa, tal y como se muestra en la Figura 3.5(a) para un parche rectangular operando en el modo fundamental $\mathrm{TM}_{10}$ y alimentado por una línea microtira. La distribución de campo eléctrico en el substrato comprendido entre el parche y el plano de masa se representa en la Figura 3.5(b). A lo largo del parche se produce una inversión de la fase del campo eléctrico, mientras que éste se mantiene constante a lo ancho del parche.

La radiación se debe principalmente a los campos existentes en los bordes en circuito abierto, denominados bordes radiantes. Puesto que la longitud del parche es aproximadamente igual a $\lambda / 2$, las componentes normales del campo eléctrico se encuentran en oposición de fase y el campo lejano correspondiente a estas componentes se cancela en la dirección broadside. Las componentes tangenciales, por el contrario, están en fase, por lo que los campos radiados resultantes se combinan para crear un máximo de radiación en la dirección broadside.

El modelo equivalente del parche puede ser por lo tanto un array de dos ranuras excitadas en fase y separadas una distancia de $\lambda / 2$, tal y como se representa en la Figura 3.5(c). Esta estructura radiaría en un hemisferio situado por encima del plano de masa.

Si se quieren tener en cuenta también las variaciones del campo eléctrico a lo largo de los bordes no radiantes será necesario recurrir a un modelo más complejo, formado por cuatro ranuras que describen el contorno del parche. 


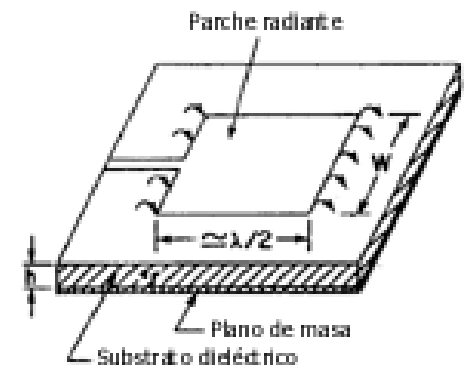

(a)

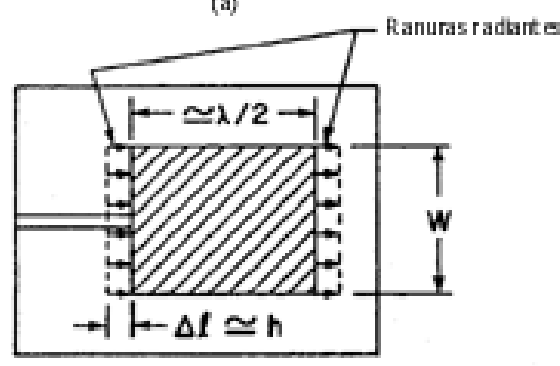

(c)

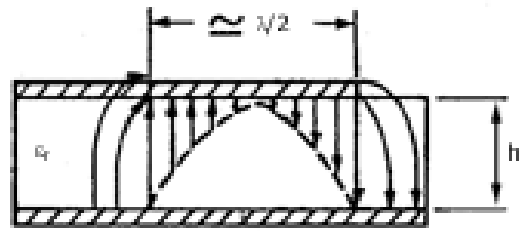

(b)

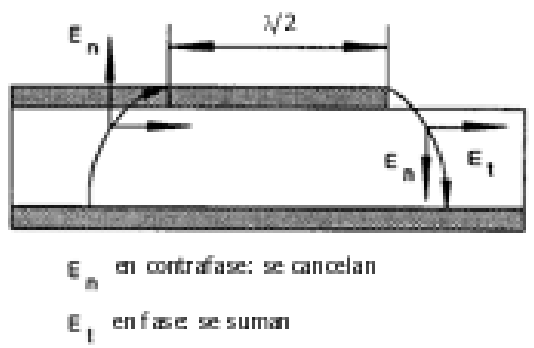

(d)

Figura 3.5: Distribución de campos en un parche rectangular.

\subsubsection{Diagramas de radiación.}

El diagrama de radiación de las antenas impresas rectangulares como la aquí descrita, si se opera en el modo fundamental $\mathrm{TM}_{10}$, es amplio en los dos planos principales, $\mathrm{E}$ y $\mathrm{H}$.

Utilizando el modelo de dos ranuras descrito en el apartado anterior se pueden calcular los campos radiados en el hemisferio situado sobre el plano de masa. Se asimila así la antena a dos distribuciones de corriente magnética lineales de anchura h (espesor del substrato) y de longitud W, separadas una distancia $\mathrm{L}$ aproximadamente igual a un medio de la longitud de onda guiada, $\lambda_{\mathrm{g}} / 2$. El campo total corresponderá pues al de un array de dos ranuras, con una separación entre elementos de $\lambda_{\mathrm{g}} / 2$. Los campos lejanos radiados son pues:

$$
\mathrm{E}_{\phi}=\underbrace{-\mathrm{j} \frac{\mathrm{Wk}_{0} \mathrm{hE} \mathrm{E}_{0} \mathrm{e}^{-\mathrm{jk} \mathrm{r}}}{2 \pi \mathrm{r}} \operatorname{sen} \theta\left(\frac{\operatorname{sen}\left(\frac{\mathrm{k}_{0} \mathrm{~h}}{2} \operatorname{sen} \theta \cos \phi\right)}{\frac{\mathrm{k}_{0} \mathrm{~h}}{2} \operatorname{sen} \theta \cos \phi}\right)\left(\frac{\operatorname{sen}\left(\frac{\mathrm{k}_{0} \mathrm{~W}}{2} \cos \theta\right)}{\frac{\mathrm{k}_{0} \mathrm{~W}}{2} \cos \theta}\right)}_{\text {Campo radiado por la ranura }} \underbrace{\cos \left(\frac{\mathrm{k}_{0} \mathrm{~L}}{2} \sin \theta \cos \phi\right)}_{\text {Factor de array }}
$$

En la ecuación (3.2) $\mathrm{hE}_{0}$ representa la tensión en la ranura, $\mathrm{V}_{0}$. Particularizando la expresión para los planos principales $\theta=\pi / 2$ y $\phi=\pi / 2$ obtenemos los campos radiados en los dos planos principales, E y H: 
Plano E:

$$
\begin{gathered}
\mathrm{E}_{\phi}=-\mathrm{j} \frac{\mathrm{Wk}_{0} \mathrm{hE}_{0} \mathrm{e}^{-\mathrm{jk} \mathrm{k}_{0}}}{2 \pi \mathrm{r}}\left(\frac{\operatorname{sen}\left(\frac{\mathrm{k}_{0} \mathrm{~h}}{2} \cos \phi\right)}{\frac{\mathrm{k}_{0} \mathrm{~h}}{2} \cos \phi}\right) \cos \left(\frac{\mathrm{k}_{0} \mathrm{~L}}{2} \cos \phi\right) \\
\mathrm{E}_{\phi}=-\mathrm{j} \frac{\mathrm{hWk_{0 }} \mathrm{E}_{0} \mathrm{e}^{-\mathrm{jk} \mathrm{r}}}{2 \pi \mathrm{r}} \operatorname{sen} \theta\left(\frac{\operatorname{sen}\left(\frac{\mathrm{k}_{0} \mathrm{~W}}{2} \cos \theta\right)}{\frac{\mathrm{k}_{0} \mathrm{~W}}{2} \cos \theta}\right)
\end{gathered}
$$

Para obtener estas expresiones, se desprecia el efecto del acoplamiento mutuo entre las dos ranuras. Sin embargo, esto no supone ninguna limitación seria en la práctica. Por otra parte, al calcular los campos radiados se ha considerado un plano de masa infinito, lo que es una fuente de error para aplicaciones en que hay que tener en cuenta la radiación trasera (relación delante-atrás, front-to$b a c k$ ), por ejemplo para eliminar interferencias. En este sentido, existe un método que permite el cálculo de los diagramas de radiación de antenas impresas en substratos de dimensiones finitas. Para ello se combina la ecuación integral del potencial mixto para parches y la forma débil de la CG-FFT (Conjugate Gradient-Fast Fourier Transform, Gradiente Conjugado-Transformada Rápida de Fourier). Hammer [3.24] propuso también un modelo basado en el concepto de apertura para calcular los campos radiados de cualquier modo excitado en un parche rectangular. Utilizando el principio de equivalencia, incluía modos de orden superior en el modelo y los cálculos, así como un substrato de tamaño finito. Para determinar la resistencia de radiación total consideraba que la resistencia de radiación de cada uno de los modos, con frecuencias de resonancia próximas entre sí, se podían combinar como un circuito paralelo.

Existen referencias igualmente al cálculo de diagramas de radiación de antenas impresas sobre substratos de dimensiones finitas utilizando la Teoría Geométrica de la Difracción combinada con el Método de los Momentos [3.25] o con el análisis de ranuras radiantes [3.26].

En lo referente a los campos próximos creados por las antenas impresas, existen diferentes trabajos que describen su distribución de forma rigurosa. Una aproximación utilizando la teoría modal de propagación de ondas desarrollada por Sommerfeld fue propuesta por Mosig y Gardiol [3.27]. La ecuación integral que se obtenía en este trabajo para la distribución de corriente no tiene solución analítica, por lo que las características de una estructura impresa ilimitada e inhomogénea se deben calcular recurriendo a métodos numéricos. Adicionalmente, Carver [3.28] estableció una fórmula para determinar la distribución de campo próximo en el perímetro de la antena, que permite estudiar cómo decae el campo al alejarse de los bordes, así como la estructura modal de los campos en la región comprendida bajo el parche. 


\subsubsection{Factor de calidad.}

El objetivo habitual del diseño cuando se trabaja con antenas impresas es lograr elementos radiantes con un gran ancho de banda y una alta eficiencia de radiación. Sin embargo estos parámetros están relacionados entre sí, y es necesario alcanzar una solución de compromiso.

Hemos visto anteriormente que una antena impresa puede considerarse, en una primera aproximación, como una cavidad resonante con pérdidas. Se puede por lo tanto calcular el factor de calidad Q asociado con las pérdidas de la estructura. Para ello se debe calcular la energía almacenada en la cavidad, incluyendo el efecto de los campos en los bordes del parche.

Cuatro mecanismos principales deben ser considerados a la hora de calcular Q:

\& las pérdidas por radiación $\left(\mathrm{Q}_{\mathrm{rad}}\right)$,

๑ las pérdidas en el substrato dieléctrico $\left(\mathrm{Q}_{\mathrm{di}}\right)$,

๑ las pérdidas en los conductores (parche, línea de alimentación y plano de masa) $\left(\mathrm{Q}_{\mathrm{cu}}\right)$,

\& las pérdidas debidas a la propagación de ondas de superficie $\left(\mathrm{Q}_{\mathrm{sw}}\right)$.

Siguiendo el procedimiento descrito por Carver [3.29], el factor de calidad Q se obtiene como:

$$
\frac{1}{\mathrm{Q}}=\frac{1}{\mathrm{Q}_{\mathrm{rad}}}+\frac{1}{\mathrm{Q}_{\mathrm{di}}}+\frac{1}{\mathrm{Q}_{\mathrm{cu}}}+\frac{1}{\mathrm{Q}_{\mathrm{sw}}}
$$

Las pérdidas por ondas de superficie son despreciables para substratos finos $(h<<\lambda)$, por lo que en estos casos la contribución de las ondas de superficie $\left(\mathrm{Q}_{\mathrm{sw}}\right)$ al factor de calidad total puede considerarse nula. En caso de utilizar substratos más gruesos o con alta permitividad, existen diferentes técnicas para calcular $\mathrm{Q}_{\mathrm{sw}}$, como por ejemplo la descrita por Richards [3.30]. Richards proporciona igualmente las fórmulas de los factores de calidad asociados a las pérdidas en los conductores $\left(\mathrm{Q}_{\mathrm{cu}}\right)$ y en los dieléctricos $\left(\mathrm{Q}_{\mathrm{di}}\right)$. Por otra parte, en [3.31] se presenta una comparación teórica de la eficiencia de las antenas alimentadas por sonda coaxial y por acoplamiento de apertura, basándose en la excitación de ondas de superficie para cada tipo de alimentación.

Leveque [3.32] desarrolló por su parte un método para simular las pérdidas dieléctricas utilizando la técnica de las diferencias finitas en el dominio del tiempo (Finite Difference-Time Domain, FDTD) y considerando un caso tridimensional. Los resultados teóricos se comparaban con los obtenidos experimentalmente para el caso de dieléctricos con y sin pérdidas, alcanzándose una buena concordancia en el caso de tener en cuenta las pérdidas en el substrato.

En el caso general, para antenas impresas sobre substratos finos, se obtienen las siguientes expresiones para los factores de calidad asociados a los dieléctricos y a los conductores:

$$
\mathrm{Q}_{\mathrm{di}}=\frac{1}{\tan \delta} \quad \mathrm{Q}_{\mathrm{cu}}=\frac{\mathrm{h}}{\mathrm{d}_{\mathrm{s}}}
$$

En estas expresiones, tan $\delta$ representa la tangente de pérdidas del substrato dieléctrico, y 
$\mathrm{d}_{\mathrm{s}}=(\pi \mathrm{f} \mu \sigma)^{-1 / 2}$ la profundidad de penetración. El efecto pelicular a altas frecuencias es de gran importancia. Existen diversos estudios que utilizan ecuaciones integrales, resueltas aplicando el método de los momentos, para determinar la resistencia en alterna y la reactancia en estos casos. Los resultados obtenidos concuerdan con algunas medidas publicadas. Se ha podido deducir que el efecto pelicular se hace apreciable para espesores de metalización inferiores a $10 \mu \mathrm{m}$.

Si la distribución del campo en la cavidad formada por el parche o en la apertura radiante equivalente es independiente del espesor del substrato, el factor de calidad ligado a la radiación viene dado por la siguiente fórmula, tal y como se describe en [3.29]:

$$
\mathrm{Q}_{\mathrm{rad}}=\frac{2 \omega \varepsilon_{\mathrm{r}} \mathrm{K}}{\mathrm{h} \frac{\mathrm{G}}{\mathrm{L}}}
$$

En esta expresión, G/L denota la conductancia por unidad de longitud de la apertura radiante y:

$$
\mathrm{K}=\frac{\iint_{\text {area }}\left|E^{2}\right| d A}{\oint_{\text {perimetro }}\left|E^{2}\right| d l}
$$

En el caso de un parche rectangular operando en el modo fundamental $\mathrm{TM}_{10}, \mathrm{~K}$ resulta ser $\mathrm{L} / 4$, mientras que $\mathrm{G} / \mathrm{L}$ equivale $\mathrm{G}_{\mathrm{rad}} / \mathrm{W}$.

Como se puede ver, el factor de calidad asociado a las pérdidas por radiación es proporcional a la inversa del espesor del substrato. Para substratos con $\mathrm{h} / \lambda>0,01, \mathrm{Q}_{\mathrm{rad}}$ será el término dominante en la fórmula global del factor de calidad. Cabe destacar también que el factor de calidad total $\mathrm{Q}$ es ligeramente mayor para una antena impresa cuadrada que para una rectangular.

\subsubsection{Directividad, ganancia, eficiencia y ancho de haz.}

Otra magnitud de interés en el estudio de antenas impresas es su directividad, definida como el cociente entre la máxima densidad de potencia radiada en el haz principal y la densidad de potencia media radiada. Si consideramos una de las ranuras radiantes equivalentes, la directividad viene dada por:

$$
\mathrm{D}=\frac{\left.\frac{1}{2} \operatorname{Re}\left(\mathrm{E}_{\theta} \mathrm{H}_{\phi}^{*}-\mathrm{E}_{\phi} \mathrm{H}_{\theta}^{*}\right)\right|_{\theta=\pi / 2}}{\mathrm{P}_{\mathrm{r}} / 4 \pi^{2}}=\frac{4 \mathrm{~W}^{2} \pi^{2}}{\mathrm{I}_{1} \lambda_{0}^{2}}
$$

Donde $\mathrm{I}_{1}$ es:

$$
I_{1}=\int_{0}^{\pi} \operatorname{sen}^{2}\left(\frac{\pi W \cos \theta}{2}\right) \tan ^{2} \theta \operatorname{sen} \theta d \theta
$$


Si consideramos ahora la antena impresa como un array de dos ranuras separadas una distancia L, la expresión de la directividad en el plano $E$ en la dirección broadside se obtiene como [3.33]:

$$
D_{W}=\frac{2 D}{1+g_{12}}
$$

Siendo $\mathrm{g}_{12}$ la conductancia mutua normalizada debida al acoplamiento entre las dos ranuras, dada por la expresión:

$$
\mathrm{g}_{12}=\frac{1}{120 \pi^{2}} \int_{0}^{\pi} \frac{\operatorname{sen}^{2}\left(\frac{\pi \mathrm{W} \cos \theta}{\lambda_{0}}\right) \tan ^{2} \operatorname{sen} \theta \mathrm{J}_{0}^{2}\left(\frac{\pi \mathrm{L}}{\lambda_{0}} \operatorname{sen} \theta\right)}{\mathrm{G}}
$$

En la ecuación (3.13), $\mathrm{J}_{0}(\mathrm{x})$ denota la función de Bessel de orden 0 y argumento $\mathrm{x}$.

Si el acoplamiento mutuo es despreciable, es decir, $\mathrm{g}_{12}<<1$, la expresión de la directividad se reduce a

$$
\mathrm{D}_{\mathrm{W}}=2 \mathrm{D}
$$

Y si:

$$
\begin{array}{ll}
\mathrm{W}<<\lambda_{0}: & \mathrm{D}_{\mathrm{W}} \cong 6,6 \\
\mathrm{~W}>>\lambda_{0}: & \mathrm{D}_{\mathrm{W}} \cong \frac{8 \mathrm{~W}}{\lambda_{0}}
\end{array}
$$

La ganancia efectiva de la antena se puede entonces calcular como:

$$
\mathrm{G}_{\mathrm{e}}=\eta \mathrm{D}_{\mathrm{W}}
$$

donde $\eta$ denota la eficiencia, definida como el cociente entre la potencia radiada y la potencia entregada a la antena, es decir:

$$
\eta=\frac{P_{\text {rad }}}{P_{r a d}+P_{c u}+P_{d i}}=\frac{Q_{\text {rad }}}{Q_{r a d}+Q_{c u}+Q_{d i}}
$$

Una formulación equivalente fue propuesta por Carver [3.29] en términos de la resistencia de entrada de la fuente:

$$
\eta=\frac{G_{\mathrm{rad}}}{\mathrm{G}_{\mathrm{in}}}=\frac{\frac{1}{\mathrm{R}_{\mathrm{rad}}}}{\frac{1}{\mathrm{R}_{\mathrm{rad}}}+\mathrm{G}_{\mathrm{cu}}+\mathrm{G}_{\mathrm{di}}}
$$

Con: 


$$
\begin{gathered}
\mathrm{C}_{10}=\frac{1}{2} \mathrm{C}_{\mathrm{dc}} \cos ^{-2}\left(\frac{\pi \mathrm{y}_{0}}{\mathrm{~L}}\right) \quad \mathrm{Q}_{\mathrm{r}}=\frac{\operatorname{Re}\left(\mathrm{k}_{10}\right)}{2 \operatorname{Im}\left(\mathrm{k}_{10}\right)} \\
\mathrm{R}_{\mathrm{rad}}=\frac{\mathrm{Q}_{\mathrm{r}}}{\omega \mathrm{C}_{10}} \\
\mathrm{G}_{\mathrm{cu}}=\mathrm{R}_{\mathrm{s}} \frac{\pi^{2} \mathrm{~W}}{2 \omega^{2} \mu^{2} \mathrm{Lh}} \quad \mathrm{G}_{\mathrm{di}}=\omega \mathrm{C}_{10} \tan \delta
\end{gathered}
$$

En estas ecuaciones, $\mathrm{k}_{10}$ representa el autovalor complejo propuesto en [3.34] y obtenido mediante un algoritmo iterativo, $C_{d c}$ es la capacitancia del parche en continua, $R_{s}=\sqrt{\mu \omega / 2 \sigma}$ es la resistencia del conductor (wave resistance), $\mathrm{G}_{\mathrm{di}} \mathrm{y} \tan \delta$ son la conductancia y la tangente de pérdidas del substrato, respectivamente, e $y_{0}$ es la inserción del punto de alimentación a lo largo del eje $y$.

La eficiencia se suele expresar en términos las pérdidas de la antena como $10 \log (1 / \eta)$, en decibelios. Para un substrato dieléctrico de permitividad $\varepsilon_{\mathrm{r}} \mathrm{y}$ espesor $\mathrm{h}$, la eficiencia aumenta con $\mathrm{h}$ y disminuye al incrementar $\varepsilon_{\mathrm{r}}$. Curvas de la eficiencia en función de la frecuencia para diferentes substratos se pueden encontrar en [3.35]. Se deduce fácilmente que la elección del substrato supondrá una importante limitación en el funcionamiento de la antena. James [3.36] sugiere que el límite inferior de la frecuencia de trabajo viene impuesto por la eficiencia $\eta$ y el ancho de banda, mientras que la relación $\mathrm{G}_{\mathrm{s}} / \mathrm{G}_{\mathrm{r}}$ establece el límite superior, siendo $\mathrm{G}_{\mathrm{r}}$ y $\mathrm{G}_{\mathrm{s}}$ las conductancias de carga debidas a las pérdidas por radiación y por ondas de superficie, respectivamente.

Según la aplicación considerada, puede ser necesario obtener anchos de haz amplios o estrechos. En general se define el ancho de haz a $-3 \mathrm{~dB}$ como la distancia angular entre las direcciones en que la ganancia de la antena decae $3 \mathrm{~dB}$, o el campo radiado se reduce a $1 / \sqrt{2}$ del valor máximo. Partiendo de las expresiones anteriores, y realizando diferentes aproximaciones, se pueden deducir las siguientes expresiones para los anchos de haz a $-3 \mathrm{~dB}$ en los planos principales de la antena:

$$
\begin{gathered}
\theta_{\mathrm{BE}}=2 \cos ^{-1}\left(\frac{7,03}{\left(3 \mathrm{~L}^{2}+\mathrm{h}^{2}\right) \mathrm{k}_{0}^{2}}\right)^{1 / 2} \\
\theta_{\mathrm{BH}}=2 \cos ^{-1}\left(\frac{1}{2+\mathrm{k}_{0} \mathrm{~W}}\right)^{1 / 2}
\end{gathered}
$$

De estas ecuaciones se desprende que el diagrama de radiación de un parche rectangular operando en el modo fundamental $\mathrm{TM}_{10}$ es ancho en ambos planos, E y H. Por otra parte, se puede aumentar el ancho de haz reduciendo $\mathrm{W}$ y L, es decir, utilizando un parche más pequeño. Para una frecuencia de resonancia determinada esto supone utilizar un substrato de mayor permitividad. Al aumentar el ancho de haz la eficiencia de la antena no varía, pero como contrapartida se reduce la ganancia, y por lo tanto la directividad. 


\subsubsection{Ancho de banda.}

Uno de los puntos críticos en el diseño de antenas impresas es el ancho de banda. En efecto, ya se ha comentado anteriormente que éste es inherentemente estrecho.

El ancho de banda de una antena se puede definir como el rango de frecuencias en el cual las características de ésta permanecen constantes. Se puede pues interpretar en diferentes términos, como por ejemplo cambios en la forma, dirección o polarización del diagrama de radiación, pérdidas de ganancia, aumento del nivel de lóbulos secundarios o cambios en la Relación de Onda Estacionaria (ROE, en inglés: Voltage Standing Wave Ratio, VSWR). La ROE se calcula a partir del coeficiente de refrlexión a la entrada de la antena, $\Gamma_{\text {in }}$, como:

$$
\mathrm{S}=\frac{1+\left|\Gamma_{\text {in }}\right|}{1-\left|\Gamma_{\text {in }}\right|}
$$

Para determinar el ancho de banda se utiliza normalmente el criterio más restrictivo, que en el caso de las antenas impresas es el referido a la ROE.

La definición tradicional del ancho de banda lo relacionaba con el factor de calidad como:

$$
\Delta \mathrm{f}=\frac{\mathrm{f}_{0}}{\mathrm{Q}}
$$

Sin embargo en esta definición no se tiene en cuenta que a menudo hay que incluir los efectos de la red de adaptación. Así pues una definición alternativa, mucho más aplicable, sería el rango de frecuencias en el que la ROE de entrada es menor que un determinado valor, normalmente 2, considerando que a la frecuencia de diseño la $\operatorname{ROE} s=1$. Si se quiere expresar este ancho de banda en términos del factor de calidad y de ROE máxima permitida se obtiene la siguiente expresión:

$$
\mathrm{BW}=\frac{\mathrm{s}-1}{\mathrm{Q} \sqrt{\mathrm{s}}}
$$

En el caso de trabajar con antenas impresas con polarización circular el ancho de banda se puede definir también como el rango de frecuencias para las cuales la relación axial se sitúa dentro de ciertos límites, normalmente $3 \mathrm{~dB}$. Richards [1.27] demostró que esta definición suele ser más restrictiva que la especificada por la impedancia, y que existe una relación entre el factor de calidad Q de la antena y el ancho de banda para el que se obtiene una buena polarización circular. Para una relación axial de $3 \mathrm{~dB}$, Richards sitúa el límite en un ancho de banda del 35/Q \%.

Un caso especial es el de las antenas impresas activas. Si en las antenas pasivas se tiene el mismo ancho de banda en términos de pérdidas de retorno y de transferencia de potencia, el problema resulta más complejo si se consideran antenas activas, ya que entonces la potencia radiada no tiene relación fija con el coeficiente de reflexión. Por lo tanto existen diferentes definiciones para el ancho de banda de las antenas activas: 
El rango en el que el coeficiente de reflexión activo $\Gamma_{\text {in }}$ está por debajo de cierto nivel y la antena entrega una potencia $\mathrm{P}_{\mathrm{A}}$ constante respecto de la frecuencia.

El rango en que se tiene una ganancia de potencia constante, despreciando la influencia del coeficiente de reflexión $\Gamma_{\text {in }}$.

En todo caso, si el objetivo es ampliar el ancho de banda de una antena, existen diferentes alternativas:

- Incrementando el espesor del substrato, hasta cierto límite para evitar la propagación excesiva de ondas de superficie

$\infty$ Utilizar substratos de baja permitividad

- Incrementar la inductancia de la estructura, mediante agujeros o ranuras, lo que puede complicar la fabricación

- Añadir componentes reactivos para reducir la ROE, complicando el diseño

\& Utilizar una línea de alimentación con dos secciones, lo que aumenta el tamaño de la estructura

Utilizar estructuras multicapa.

Para no tener que recurrir a estas técnicas se ha dedicado mucho esfuerzo a buscar estructuras con un gran ancho de banda, o resonantes a diferentes frecuencias.

\subsubsection{Polarización.}

La polarización de una antena impresa rectangular es normalmente lineal. Sin embargo, en muchas aplicaciones es deseable una antena impresa operando con una polarización circular. En este sentido se han llevado a cabo numerosos estudios para desarrollar elementos sencillos de polarización circular. Una manera de lograrlo sería excitar dos modos ortogonales degenerados de igual amplitud. Los campos radiados por cada uno de los modos serían entonces perpendiculares entre sí, y polarizados ortogonalmente.

\subsubsection{Ondas de superficie.}

En determinadas condiciones se puede dar, al trabajar con antenas impresas, una radiación espuria en la dirección endfire debida a la propagación de ondas de superficie. Este hecho puede resultar perjudicial para los diagramas de radiación, sobre todo si se requieren niveles de lóbulos secundarios bajos, o un bajo nivel de polarización cruzada.

Las ondas de superficie se componen de modos TE y TM que se propagan por el substrato más allá de la región delimitada por el parche. Estas ondas se atenúan en la dirección normal al plano de la antena, y su velocidad de fase está fuertemente ligada a la constante dieléctrica y al espesor del 
substrato.

La frecuencia de corte del modo de orden inferior, $\mathrm{TM}_{0}$ es igual a 0 , por lo que este modo se excita siempre, incluso en substratos muy finos con constantes dieléctricas bajas. Su velocidad de fase es próxima a la velocidad de la luz. La excitación de esta onda se hace apreciable cuando $\mathrm{h} / \lambda_{0}>0,09$ para $\varepsilon_{\mathrm{r}} \approx 2,3 \mathrm{y} \mathrm{h} / \lambda_{0}>0,03$ para $\varepsilon_{\mathrm{r}} \approx 10$. Si nos limitamos al caso de antenas rectangulares, un posible criterio para evitar la excesiva propagación de ondas de superficie sería el propuesto por Wood [3.37]: $\mathrm{h} / \lambda_{0}<0,07$ para $\varepsilon_{\mathrm{r}} \approx 2,3 \mathrm{y} \mathrm{h} / \lambda_{0}<0,023$ para $\varepsilon_{\mathrm{r}} \approx 10$.

Para los modos de orden superior $\mathrm{TE}_{\mathrm{n}} \mathrm{y} \mathrm{TM}_{\mathrm{n}}$ la frecuencia de corte se determina como:

$$
\mathrm{f}_{\mathrm{c}}=\frac{\mathrm{nc}}{4 \mathrm{~h} \sqrt{\varepsilon_{\mathrm{r}}-1}}
$$

Siendo c la velocidad de la luz en el vacío y $n=1,3,5 \ldots$ para los modos $\mathrm{TE}_{\mathrm{n}}$ y $\mathrm{n}=2,4,6 \ldots$ para los modos $\mathrm{TM}_{\mathrm{n}}$.

Cuando una onda quasi-TEM se propaga a lo largo del parche con una velocidad de fase próxima a la de la onda de superficie se produce un acoplamiento indeseado entre ambas. La mínima frecuencia a la que se puede dar este acoplamiento determina la frecuencia máxima de trabajo de la antena.

Hemos visto anteriormente que una opción para incrementar el ancho de banda de la antena consiste en aumentar el espesor del substrato. La propagación excesiva de ondas de superficie representa pues un límite práctico para esta técnica. Bhattacharyya [3.38] propuso unos diseños en los que no que no se excitaba el modo dominante $\mathrm{TM}_{0}$ de las ondas de superficie. Con ello lograba una eficiencia del 100\%. Sus diseños se basan en el hecho de que un anillo circular de corriente magnética, con un radio adecuado, no excita el modo de ondas de superficie.

\subsection{ANTENAS ELÉCTRICAMENTE PEQUEÑAS.}

A lo largo de este trabajo, las antenas con las que vamos a trabajar pueden incluirse en la categoría de antenas eléctricamente pequeñas, por lo que resulta interesante revisar las limitaciones que esto implica.

El concepto de antena eléctricamente pequeña se utiliza para denominar aquellas antenas que se pueden inscribir completamente en una esfera de radio $\mathrm{a}=1 / \mathrm{k}$, donde $\mathrm{k}=2 \pi / \lambda$ representa el número de onda asociado al campo electromagnético, según lo establecido por Wheeler [3.39]. Wheeler relacionaba por así las limitaciones en el funcionamiento de una antena con el volumen ocupada por ésta a través del factor de potencia radiada (radiation power factor). 
Estas antenas eléctricamente pequeñas se caracterizan por una resistencia de radiación mucho menor que la reactancia, y por unos diagramas de radiación independientes del tamaño de la antena [3.40].

\subsubsection{Límites teóricos de las antenas eléctricamente pequeñas.}

El criterio más conocido es el propuesto por Chu [3.41], y recogido por Harrington [3.42]. Las antenas se analizan aquí utilizando la teoría de modos esféricos. La potencia radiada se puede calcular a partir de los modos que se propagan en el interior de la esfera. Si la esfera es lo suficientemente grande como para que se propaguen varios modos, este enfoque resulta complicado de utilizar, puesto que resulta difícil calcular los coeficientes de los diferentes modos [3.43]. La ventaja de este método es que permite relacionar de forma intuitiva el mínimo factor de calidad que se puede obtener con la dimensión máxima de la antena. Para ello se aplica la siguiente expresión analítica aproximada:

$$
\mathrm{Q}=\frac{1+3(\mathrm{ka})^{2}}{(\mathrm{ka})^{3}\left[1+(\mathrm{ka})^{2}\right]}
$$

Para llegar a esta expresión, se considera que los modos de orden superior son evanescentes para $\mathrm{ka}<1$.

Sin embargo, McLean [3.44] cuestiona la exactitud de la fórmula, achacándolo a un error algebraico, y propone la siguiente alternativa:

$$
\mathrm{Q}=\frac{1+2(\mathrm{ka})^{2}}{(\mathrm{ka})^{3}\left[1+(\mathrm{ka})^{2}\right]}
$$

Ambas expresiones concuerdan en el límite inferior de ka, sin embargo la diferencia crece al acercarse a ka=1, límite superior de lo que se considera antenas eléctricamente pequeñas. En ambas expresiones se considera un único modo de propagación. Si se tuvieran dos modos, el factor de calidad se reduce a la mitad.

Del mismo modo, se ha asumido que la antena tiene una eficiencia $\eta$ del $100 \%$, es decir, que las antenas no tienen pérdidas. Si las tuviera, el efecto sería el de una resistencia en serie con la resistencia de radiación.

En la Figura 3.6 se muestran las curvas de factor de calidad en función de ka para diferentes valores de eficiencia, calculadas utilizando la fórmula de dada en la ecuación (3.29).

En [3.44] McLean justifica igualmente la necesidad de obtener una expresión exacta de factor de calidad. La fórmula a la que llega, para el caso de una antena con polarización lineal, en la que se excita un solo modo, es: 


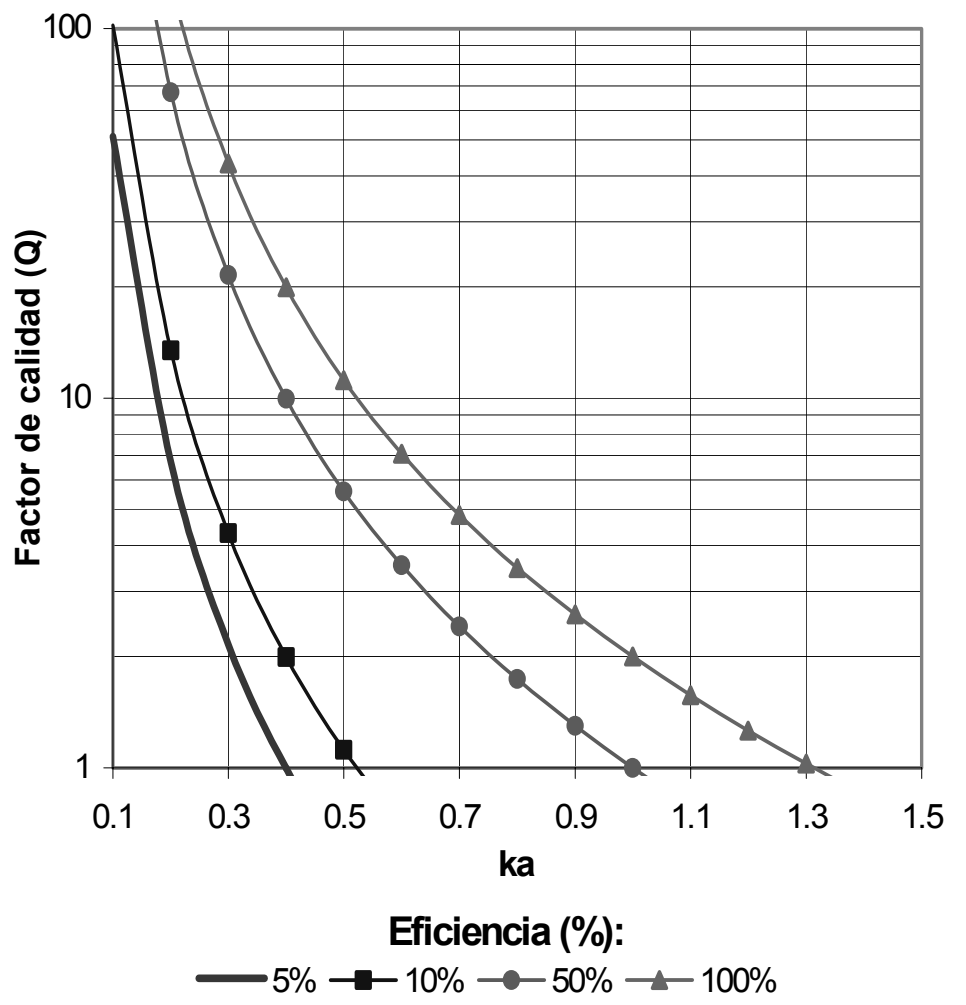

Figura 3.6: Límite teórico de Chu-Harrington: factor de calidad vs. tamaño de la antena, para antenas monomodo y diferentes valores de eficiencia.

$$
\mathrm{Q}=\frac{1}{(\mathrm{ka})^{3}}+\frac{1}{\mathrm{ka}}
$$

En [3.40] se propone tener en cuenta además la eficiencia de la antena, incluyéndola en (3.31) para obtener la siguiente expresión:

$$
\mathrm{Q}=\left(\frac{1}{(\mathrm{ka})^{3}}+\frac{1}{\mathrm{ka}}\right) \eta
$$

En la Figura 3.7 se muestra una comparación entre los límites obtenidos con las tres ecuaciones, (3.29), (3.30) y (3.31). Se puede apreciar que para valores de ka pequeños los resultados son similares en los tres casos. Sin embargo, las diferencia se hace significativa entre los resultados obtenidos utilizando la expresión derivada por McLean y la de Chu-Harrington corregida para valores de ka cercanos a 1 .

En cualquier caso, para valores pequeños de $\mathrm{ka}(\mathrm{ka}<<1)$, el factor de calidad mínimo alcanzable es aproximadamente:

$$
\mathrm{Q} \approx \frac{1}{(\mathrm{ka})^{3}}
$$

El principal inconveniente del método de Chu reside en que está restringido a modos esféricos. Collin [3.45] presentó un método de análisis del factor de calidad general, que permite hacer 
extensible el análisis a modos de orden superior, tanto esféricos como cilíndricos. El método consiste básicamente en sustraer de la densidad de energía del campo total la parte correspondiente a los campos radiados.

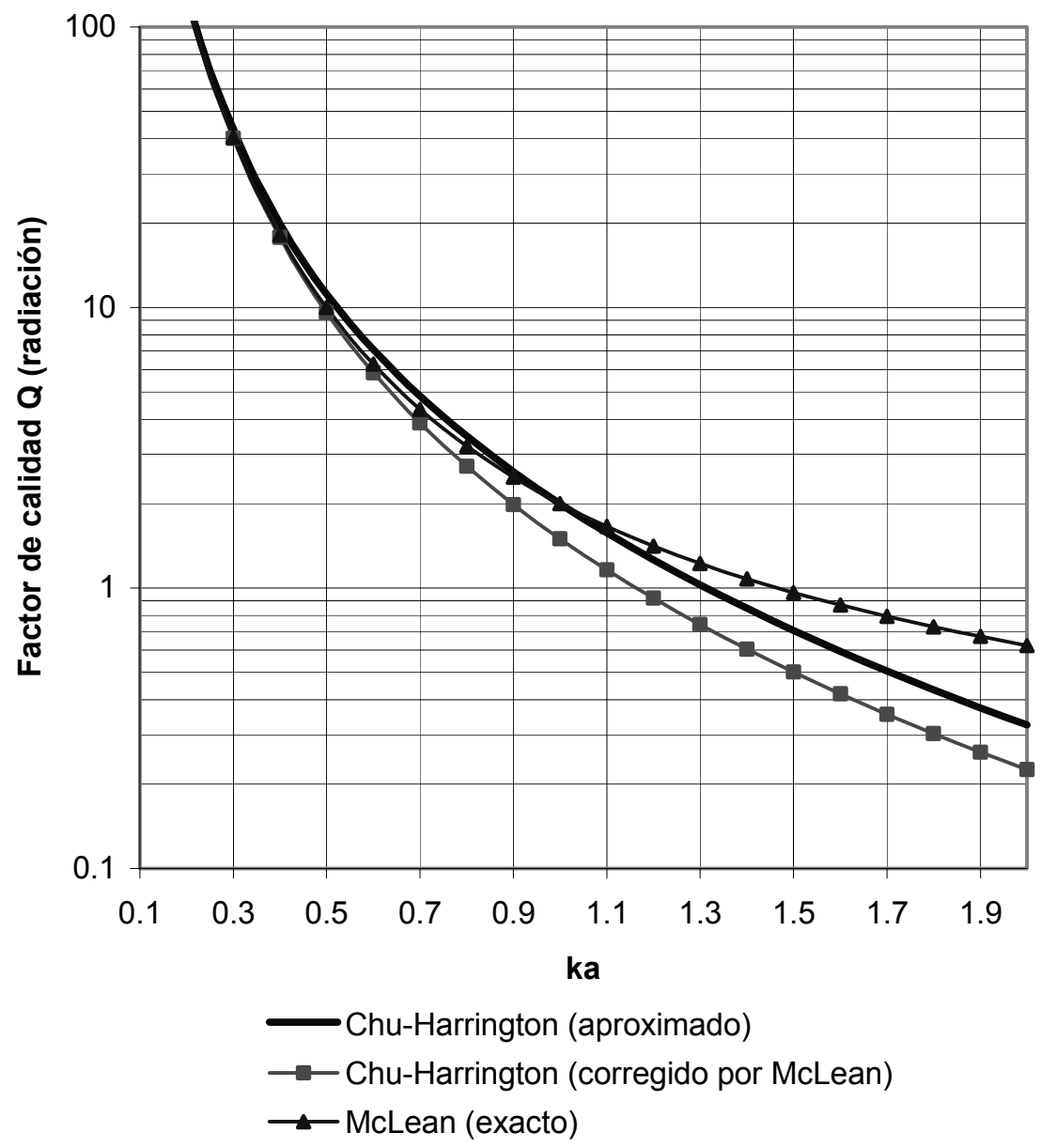

Figura 3.7: Comparación de los límites teóricos obtenidos utilizando las fórmulas de Chu-Harrington y de McLean.

\subsubsection{Ancho de banda y ganancia.}

A partir de los resultados anteriores, se puede establecer igualmente el límite de ancho de banda para una antena. Si consideramos la definición clásica de ancho de banda como la distancia entre las frecuencias en que la potencia es igual a un medio de la potencia máxima, el ancho de banda relativo vendrá dado por [3.43]:

$$
\mathrm{BW}=\frac{\Delta \mathrm{f}}{\mathrm{f}_{\mathrm{c}}}=\frac{\mathrm{f}_{\text {up }}-\mathrm{f}_{\text {low }}}{\mathrm{f}_{\mathrm{c}}}=\frac{1}{\mathrm{Q}}
$$

Donde $f_{c}$ representa la frecuencia central de la banda de interés, $\Delta f$ el ancho de banda total, y $f_{\text {up }}$ y $\mathrm{f}_{\text {low }}$ los límites de la banda.

Esta expresión se cumple para valores altos de Q, pero resulta inexacta para $\mathrm{Q}<2$. Sin embargo 
estas curvas siguen siendo útiles para hacerse una idea de los anchos de banda alcanzables para una determinada antena. Para un tamaño de antena dado, el aumento del ancho de banda para una determinada frecuencia sólo se puede obtener pues optimizando la utilización del volumen disponible para establecer un modo TE y un modo TM, reduciendo así el factor de calidad Q a la mitad, o disminuyendo la eficiencia de la antena.

Para $\mathrm{ka}<<1$, el límite inferior del ancho de ancho de banda corresponde aproximadamente a $(\mathrm{ka})^{3}$, tal y como se ilustra en la Figura 3.8. Este resultado es independiente de la tecnología utilizada para construir la antena.

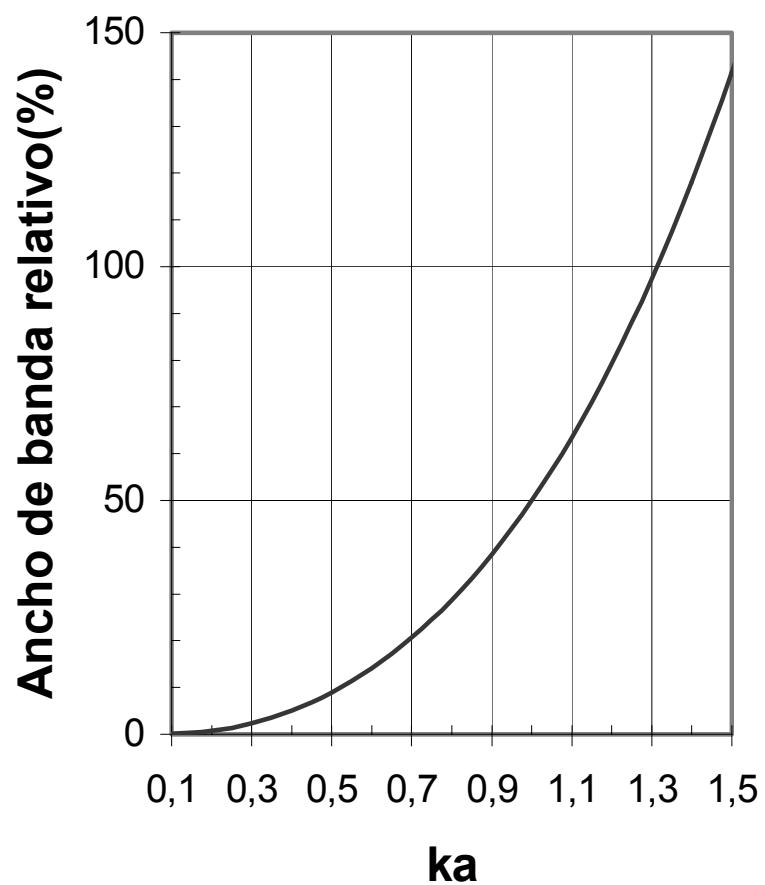

Figura 3.8: Límite teórico del ancho de banda.

Del mismo modo, Harrington [3.42] propone una fórmula para determinar el límite máximo de la ganancia de una antena para que ésta conserve un ancho de banda razonable:

$$
\mathrm{G}=(\mathrm{ka})^{2}+2 \mathrm{ka}
$$

La curva de ganancia máxima alcanzable se representa en la Figura 3.9.

En todo caso, de los resultados anteriores se deduce que el ancho de banda de una antena, contenida en una esfera de radio a, se puede mejorar si se utiliza eficientemente el volumen disponible dentro de la esfera [3.46].

Finalmente, Siwiak [3.47] propone una expresión que relaciona el ancho de banda relativo con la eficiencia de radiación como:

$$
\mathrm{BW}=\frac{2(\mathrm{ka})^{3}}{\eta}
$$




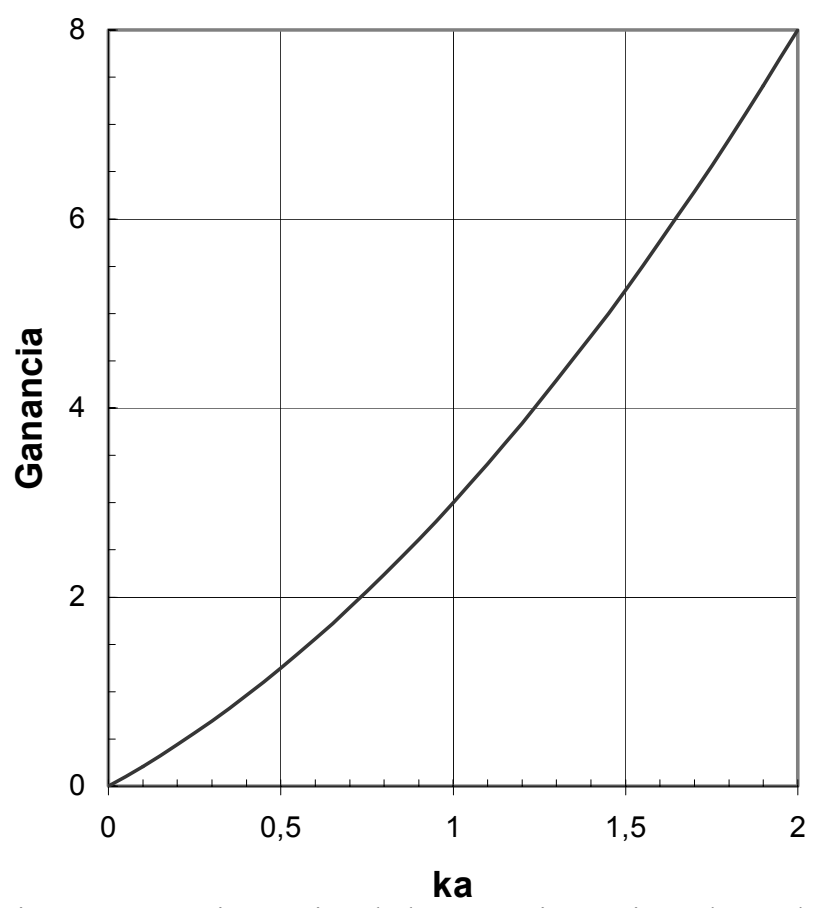

Figura 3.9: Límite teórico de la ganancia máxima alcanzable.

La ecuación (3.36) implica que, si el tamaño de antena es fijo, se puede aumentar el ancho de banda en detrimento de la eficiencia de radiación. Los campos reactivos se mantienen en las cercanías de la antena, pero se extienden a una distancia superior al radio a de la esfera que la contiene. Por lo tanto, los objetos situados en esta zona interactuarán fuertemente con la antena, y se pueden producir pérdidas adicionales en el rendimiento del sistema.

\subsection{Biblografía.}

[3.1] Deschamps, G.A., 'Microstrip Microwave Antennas', 3rd USAR Symp. on Antennas, 1953.

[3.2] Greig, D.D. y Engleman, H.F., 'Microstrip - a New Transmission Technique for the Kilomegacycle Range', Proceedings of the IRE, 1952, vol. 40, pp. 1644-1650.

[3.3] Lewin, L., 'Radiation from Discontinuities in Stripline', Proceedings of the IEE, vol. 107C, 1960, pp. 163-170.

[3.4] Bahl, I.J. y Bhartia, P., 'Microstrip Antennas', Artech House, Dedham, MA, Estados Unidos, 1980.

[3.5] James, J.R., Hall, P.S. y Wood, C., 'Microstrip Antenna Theory and Design', IEE, Peter Peregrinus Ltd, Londres, 1981.

[3.6] 'Handbook of Microstrip Antennas', editado por J.R. James y P.S. Hall, Peter Peregrinus Ltd, para IEE, vol. 1 y 2, Londres, 1989. 
[3.7] 'Analysis, Design and Measurement of Small and Low-Profile Antennas', editado por K. Hirasawa y M. Haneishi, Artech House, Norwood, MA, 1992.

[3.8] 'Advances in Microstrip and Printed Antennas', editado por K.F. Lee y W. Chen, John Wiley \& Sons, Nueva York, 1997.

[3.9] Pichon, P. Mosig, J.R. Papiernik, A., 'Input Impedance of Arbitrarily Shaped Microstrip Antennas', Electronics Lett., 1988, vol. 24, n 19, pp. 1214-1215.

[3.10] Sipus, Z., Bartolic, J. y Stipetic, B., 'Input Impedance of Rectangular Patch Antenna Fed by Microstrip Line', Electronic. Lett., 1992, vol. 28, n² 20, pp. 1886-1887.

[3.11] Hall, R.C. y Mosig, J.R., 'Rigorous Feed Model for Coaxially Fed Microstrip Antenna', Electronics Lett., 1990, vol. 26, n 1, pp. 64-66.

[3.12] Sipus, Z., Bartolic, J. y Stipetic, B., 'Simple Model for the Input Impedance of Coax-Fed Rectangular Microstrip Patch Antenna for CAD', IEE Proceedings, Part H, 1988, vol. 135, $\mathrm{n}^{\circ}$ 5, pp. 323-326.

[3.13] Gao, F. y Yang, Q.J., 'Effect of Substrate on Current Distribution of Feeding Probe', Electronics Lett., 1988, vol. 24, n 7, pp. 374-376.

[3.14] Bahl, I.J., Bhartia, P. y Stuchly, S.S., 'Microstrip Antennas Covered with a Dielectric Layer', IEEE Antennas and Propagat. Society Symp., 1980, pp. 589-592.

[3.15] Chen, W., Lee, K.F., Dahele, J.S. y Lee, R.Q., 'CAD Formula for the Resonant Frequency of Rectangular Patch Antenna with Dielectric Cover', 8th IEE Int. Conf. on Antennas \& Propagat., 1993, pp. 550-553.

[3.16] Pozar, D.M., 'Microstrip Antenna Aperture-Coupled to a Microstripline', Electronics Lett., 1985, vol. $21, \mathrm{n}^{\circ} 2$, pp. 49-50.

[3.17] Gao, X. y Chang, K., 'Network Modelling of an Aperture Coupling Between Microstrip Line and Patch Antenna for Active Array Applications', IEEE Trans. on Microwave Theory and Tech., 1988, vol. 36, $\mathrm{n}^{\circ}$ 3, pp. 505-513.

[3.18] Sullivan, P. y Schaubert, D.H., 'Analysis of an Aperture Coupled Microstrip Antenna', IEEE Trans. on Antennas and Propagat., 1986, vol. 34, pp. 977-984.

[3.19] Buck, A.C. y Pozar, D.M., 'Aperture-Coupled Microstrip Antenna with a Perpendicular Feed', Electronics Lett., 1986, vol. 22, n 3, pp. 125-126.

[3.20] Pozar, D.M. y Targonski, S.D., 'Improved Coupling for Aperture Coupled Microstrip Antennas', Electronics Lett., 1991, vol. 27, n 13, pp. 1129-1131.

[3.21] Rostan, F., Gottwald, G. y Heidrich, E., 'Wideband Aperture-Coupled Microstrip Patch Array for Satellite TV Reception', 8th IEE Int. Conf. on Antennas \& Propagat., 1993, pp. 190-193.

[3.22] Chung, M.H. y Sangwook, N., 'Analysis of a Rectangular Patch Antenna Coupled by 
Microstrip Feedline Gap Discontinuity', IEEE Antennas and Propagat. Society. Symp., 1993, pp. 1236-1239.

[3.23] Waterhouse, R.B. y Novak, D., 'Possible Configurations for Optically Fed Microstrip Patch Arrays and their Scan Performances', IEEE Antennas and Propagat. Society. Symp., 1993, pp. 1232-1235.

[3.24] Hammer, D., Van Bouchaute, D., Verschraeben, D. y Van de Capelle, A., 'Model for Calculating the Radiation Field of Microstrip Antennas', IEEE Trans. on Antennas and Propagat., 1979, vol. 27,n², pp. 267-270.

[3.25] Aas, J.A. y Jakobsen, K., 'Radiation Patterns of Rectangular Microstrip Antennas on Finite Ground Planes', 5th European Microw. Conf., 1975, pp. 384-389.

[3.26] Huang, J. 'The Finite Ground Plane Effect on the Microstrip Antenna Radiation Patterns', IEEE Trans. on Antennas and Propagat., 1983, vol. 31, $\mathrm{n}^{\circ}$ 4, pp. 649-653.

[3.27] Mosig, J.R. y Gardiol, F.E., 'The Near Field of an Open Microstrip Structure', IEEE Antennas and Propagat. Society. Symp., 1979, pp. 379-382.

[3.28] Carver, K.R., 'Calculated and Measured Perimeter Field Distributions for Microstrip Antennas', IEEE Antennas and Propagat. Society. Symp., 1980, pp. 727-730.

[3.29] Carver, K.R y Mink, J.W., 'Microstrip Antenna Technology', IEEE Trans. on Antennas and Propagat., 1981, vol. 29, $\mathrm{n}^{\circ}$ 1, pp. 2-23.

[3.30] Hammer,D., Van Bouchaute, D., Verschraeben, D. y Van de Capelle, A., 'Theory and Application for Microstrip Antennas', Proc. Workshop on Printed Circuit Antenna Tech., New Mexico State Univ., Las Cruces, Oct. 1979, pp. 8/1-23.

[3.31] Herscovici, N. y Aberle, J.T., 'Surface Wave Efficiency of Microstrip Antennas', IEEE Antennas and Propagat. Society. Symp., 1993, pp. 638-641.

[3.32] Leveque, Ph., Reineix, A. y Jecko, B., 'Modelling of Dielectric Losses in Microstrip Patch Antennas: Application of FDTD Method', Electronics Lett., 1992, vol. 28, n 6, pp. 539541.

[3.33] Derneryd, A.G., 'A Theoretical Investigation of the Rectangular Microstrip Antenna Element', IEEE Trans. on Antennas and Propagat., 1978, vol. 26, n 4, pp. 532-535.

[3.34] Carver, R.J., 'Practical Analytical Techniques for the Microstrip Antenna', Proc. Workshop on Printed Circuit Antenna Tech., New Mexico State Univ., Las Cruces, Oct. 1979, pp. 7/1-20.

[3.35] Milligan, T.A., 'Bandwidth and Efficiency of a Microstrip Patch Antenna', IEEE Antennas and Propagat. Society. Symp., 1980, pp. 585-588.

[3.36] James, J.R., Hall, P.S., Wood, C. y Henderson, A., 'Some Recent Developments in Microstrip Antenna Design', IEEE Trans. on Antennas and Propagat., 1981, vol. 29, n 1, pp. 124-128. 
[3.37] Wood, C., 'Analysis of Microstrip Circular Patch Antennas', Proceedinsg of the IEE., 1981, vol. 128H, pp. 69-76.

[3.38] Bhattacharyya, A.K., Jackson, D.R., Williams, J.T. y Smith, R., 'Microstrip Patch Designs Which Do Not Excite Surface Waves', IEEE Antennas and Propagat. Society. Symp., 1991, pp. 68-71.

[3.39] Wheeler, H.A., 'Fundamental Limitations of Small Antennas', Proceedings of the IRE, 1947, vol. 35, pp. 1479-1484.

[3.40] Stutzmann, W.L. y Thiele, G.A., 'Antenna Theory and Design', $2^{\text {nd }}$ Edition, John Wiley \& Sons, 1998.

[3.41] Chu, L.J., 'Physical Limitations of Omni-Directional Antennas', J. Appl. Phys., 1948, vol. 19, pp. 1163-1175.

[3.42] Harrington, R.F., 'Effect of Antenna Size on Gain, Bandwidth and Efficiency', J. Res. Nat. Bur. Stand., 1960, vol. 64D, pp. 1-12.

[3.43] Hansen, R.C., 'Fundamental Limitations in Antennas', Proceedings of the IEEE, 1981, vol. $69, \mathrm{n}^{\circ} 2$, pp. $170-182$.

[3.44] McLean, J.S., 'A Re-Examination of the Fundamental Limits on the Radiation Q of Electrically Small Antennas', IEEE Trans. on Antennas and Propagat., 1996, vol. 44, $\mathrm{n}^{\circ}$ 5, pp. 672-676.

[3.45] Collin, R.E. y Rothschild, S., 'Evaluation of Antenna Q', IEEE Trans. on Antennas and Propagat., 1964, vol. 47, nº 1, pp. 23-27.

[3.46] Balanis, C.A., 'Antenna Theory, Analysis and Design', $2^{\text {nd }}$ Edition, John Wiley \& Sons, 1997.

[3.47] Siwiak, K., 'Radiowave Propagation and Antennas for Personal Communications', Artech House, Norwood, MA, 1995. 



\section{Capítulo 4: MÉTOdOS DE ANÁLISIS DE ANTENAS IMPRESAS.}

En teoría, no existe diferencia entre teoría y práctica; en la práctica sí la hay. Jan L.A. van de Snepscheut

\subsection{INTRODUCCIÓN.}

Para caracterizar completamente una antena es necesario obtener su impedancia de entrada, ancho de banda, ganancia, diagrama de radiación, eficiencia y factor de calidad. En el caso de las antenas impresas, existen diferentes métodos, que se dividen en dos grandes categorías:
Métodos analíticos.
\& Métodos numéricos.

Los métodos analíticos parten de una serie de aproximaciones referentes al mecanismo de radiación de la antena para permitir la resolución del problema. Para facilitar el trabajo, se suelen despreciar fenómenos como la propagación de ondas de superficie, los fenómenos de dispersión, la continuidad de la corriente en la transición entre el parche y la línea de alimentación, o la interacción entre elementos de un array. Con ello se pretende obtener expresiones analíticas relativamente sencillas, aunque éstas sean válidas únicamente en un estrecho margen de frecuencias. Por lo tanto resulta prácticamente imposible su extensión a elementos radiantes de forma arbitraria.

Por el contrario, los métodos numéricos pueden ser utilizados para el análisis de antenas de forma arbitraria, para las que resulta imposible predecir la distribución superficial de corriente en el parche. Permiten además considerar sustratos gruesos en relación con la longitud de onda, teniendo en cuenta el efecto de las ondas de superficie, los campos evanescentes o el acoplamiento mutuo entre diferentes parches. Sin embargo estos métodos numéricos requieren una gran capacidad de computación, puesto que se basan en la resolución de ecuaciones formuladas con gran rigor.

Estos métodos numéricos, combinados con las técnicas de CAD, resultan esenciales para el diseño de antenas impresas. Los simuladores electromagnéticos $(\mathrm{em})$ han avanzado a gran velocidad, al implementar algoritmos de resolución más eficientes. Hoy en día es posible encontrar programas de 
CAD em que permiten llevar a cabo el desarrollo de antenas utilizando únicamente la capacidad de cálculo de un ordenador personal.

\subsection{MÉtodos anALíticos.}

A continuación se describen algunos métodos analíticos desarrollados para el diseño de antenas impresas, empezando por los más rigurosos para ver a continuación técnicas más sencillas. En situaciones prácticas son estas últimas las más utilizadas, ya que permiten obtener soluciones bastante aproximadas sin necesidad de cálculos excesivamente complicados [4.1].

\subsubsection{Método del potencial vector.}

El método del potencial vector proporciona una solución rigurosa de la radiación de una antena impresa rectangular [4.2]. Para ello se recurre a la teoría de propagación de ondas de Sommerfeld para calcular el campo creado por un dipolo eléctrico horizontal impreso sobre un substrato de espesor h y permitividad $\varepsilon_{0}$ como el representado en la Figura 4.1 [4.1]. Las características de la antena impresa se calculan mediante técnicas numéricas.

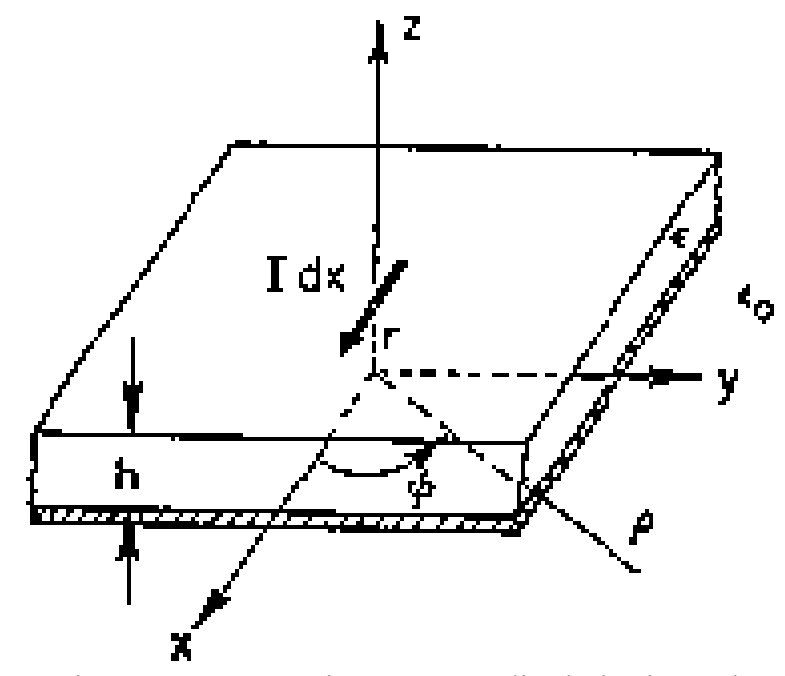

Figura 4.1: Antena impresa con dipolo horizontal.

Los campos se pueden determinar a partir del potencial vector $\vec{A}$. Para cumplir las condiciones de contorno en el interfaz aire-dieléctrico, las componentes en x y z deben satisfacer la ecuación de Helmholtz inhomogénea:

$$
\left(\nabla^{2}+\mathrm{k}^{2}\right) \overrightarrow{\mathrm{A}}=-\mu \overrightarrow{\mathrm{J}}=-\hat{\mathrm{x}} \mu \mathrm{Id} \times \delta(\overrightarrow{\mathrm{r}})
$$

Donde $\mu$ es la permeabilidad del substrato, k el número de onda y $\delta(\overrightarrow{\mathrm{r}})$ la función delta de Dirac. 
Los campos radiados se obtienen a partir del vector potencial $\overrightarrow{\mathrm{A}}$ como:

$$
\begin{gathered}
\overrightarrow{\mathrm{H}}=\frac{1}{\mu} \nabla \times \overrightarrow{\mathrm{A}} \\
\overrightarrow{\mathrm{E}}=\frac{1}{\mathrm{j} \omega \mu \varepsilon}\left[\mathrm{k}^{2} \overrightarrow{\mathrm{A}}+\nabla(\nabla \cdot \overrightarrow{\mathrm{A}})\right]
\end{gathered}
$$

A partir del dipolo eléctrico se obtiene la distribución superficial de corriente como:

$$
\overrightarrow{\mathrm{J}}_{\mathrm{S}}=\hat{\mathrm{x}} \frac{\mathrm{Idx}}{2 \pi} \int_{0}^{\infty} \mathrm{J}_{0}(\xi \rho) \xi \mathrm{d} \xi
$$

$\mathrm{J}_{0}(\xi \rho)$ representa la función de Bessel de orden $0 \mathrm{y}$ argumento $\xi \rho$.

Las condiciones de contorno en el interfaz aire-dieléctrico permiten calcular las componentes en $x$ y $z$ del vector potencial $\vec{A}$ en las dos regiones de interés. Sustituyendo en las ecuaciones (4.2) y (4.3) se pueden calcular los campos electromagnéticos en cualquier punto del espacio, y determinar así las características de la antena.

En una antena impresa, $\mathrm{H}_{\mathrm{z}}=0$ en el parche conductor. Aplicando esta condición se obtiene:

$$
\mathrm{H}_{\mathrm{z}}=\frac{\mathrm{Idx}}{2 \pi} \operatorname{sen} \phi \int_{0}^{\infty} \mathrm{J}_{1}(\xi \rho) \frac{\xi^{2}}{\mathrm{~F}_{\mathrm{x}}} \mathrm{d} \xi, \quad \mathrm{z}=\mathrm{h}
$$

Con:

$$
\mathrm{F}_{\mathrm{x}}=\beta_{0}+\beta \operatorname{coth}(\beta \mathrm{h}) \quad \beta_{0}^{2}=\xi^{2}-\mathrm{k}_{0}^{2} \quad \beta^{2}=\xi^{2}-\varepsilon_{\mathrm{r}} \mathrm{k}_{0}^{2}
$$

De esta forma se puede establecer una representación integral de la distribución de corriente en el parche y de la frecuencia de resonancia. Sin embargo, la ecuación (4.5) no tiene solución analítica y debe ser calculada utilizando métodos numéricos, realizando las debidas simplificaciones para garantizar la convergencia.

\subsection{2. $\quad$ Modelo de malla de hilos.}

Otro método de análisis de antenas impresas es el modelo de malla de hilos (wire grid model) propuesto por Agrawal [4.3]. Este método es una combinación de un enfoque numérico y uno empírico de la antena impresa, cualquiera que sea su geometría. La antena se representa como una malla de segmentos conductores, tal y como se muestra en la Figura 4.2.

Si se logra determinar el valor de la corriente en cada uno de los segmentos es posible establecer las características de la antena. Para realizar el análisis se sustituye el plano de masa por la imagen del parche radiante, tal y como se muestra en la Figura 4.3. 

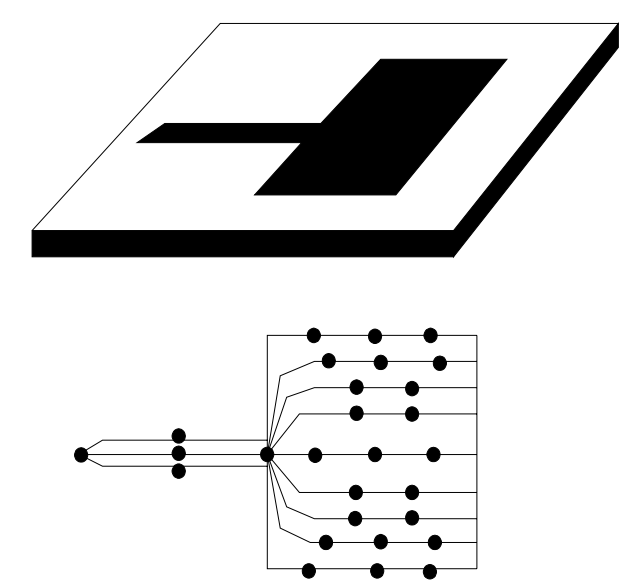

Figura 4.2: Modelo de la antena impresa como una malla de hilos.

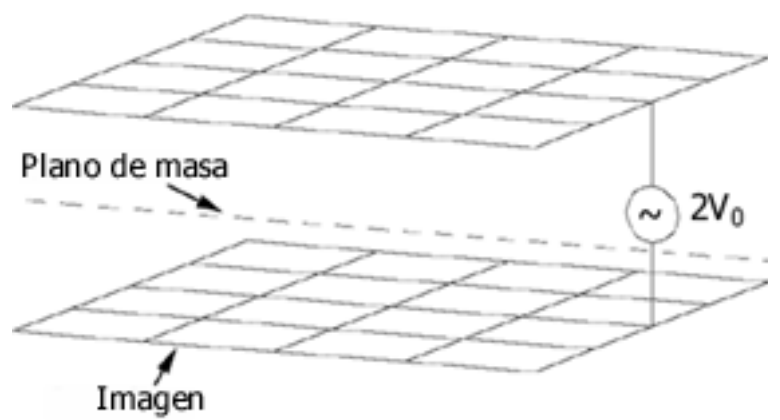

Figura 4.3: Modelo de la antena y su imagen.

La malla considerada está compuesta por $\mathrm{n}$ dipolos superpuestos, por cada uno de los cuales fluye una corriente $\mathrm{I}_{\mathrm{i}}$, que se anula en los extremos. Las corrientes en la malla se obtienen utilizando el teorema de la reacción de Richmond:

$$
\left[\mathrm{Z}_{\mathrm{ij}}\right]\left[\begin{array}{c}
\mathrm{I}_{1} \\
\mathrm{I}_{2} \\
\cdot \\
\cdot \\
\mathrm{I}_{\mathrm{n}}
\end{array}\right]=\left[\begin{array}{c}
2 \mathrm{~V}_{0} \\
0 \\
\cdot \\
\cdot \\
0
\end{array}\right]
$$

$\mathrm{Z}_{\mathrm{ij}}$ es la impedancia mutua ente los elementos i y $\mathrm{j}, \mathrm{y} \mathrm{I}_{1}$ la corriente del elemento conectado al generador.

Resolviendo este sistema de $n$ ecuaciones se obtiene la corriente en cada segmento y se pueden deducir por lo tanto las características de la antena, aunque con un factor de escala. Dicho factor se puede determinar estudiando el comportamiento de la antena si el substrato dieléctrico se extiende a todo el semiespacio situado sobre el plano de masa.

Este método permite obtener excelentes resultados si la malla es lo suficientemente fina. Sin embargo, esto supone incrementar el tiempo de cálculo y el espacio de memoria necesario para llevar a cabo los cálculos. La malla utilizada por Agrawal es una simple imagen de la corriente en el parche radiante. Es por lo tanto posible hallar otras mallas que permitan mejorar los tiempos de 
cálculo y los resultados.

\subsubsection{Modelo de cavidad.}

Otra forma de análisis de antenas impresas es el modelo de cavidad [4.4]. Se considera entonces que la región comprendida entre el parche y el plano de masa es una cavidad, con una pared magnética a lo largo del perímetro del parche, paredes eléctricas en las partes superior e inferior, tal y como se ilustra en la Figura 4.4.

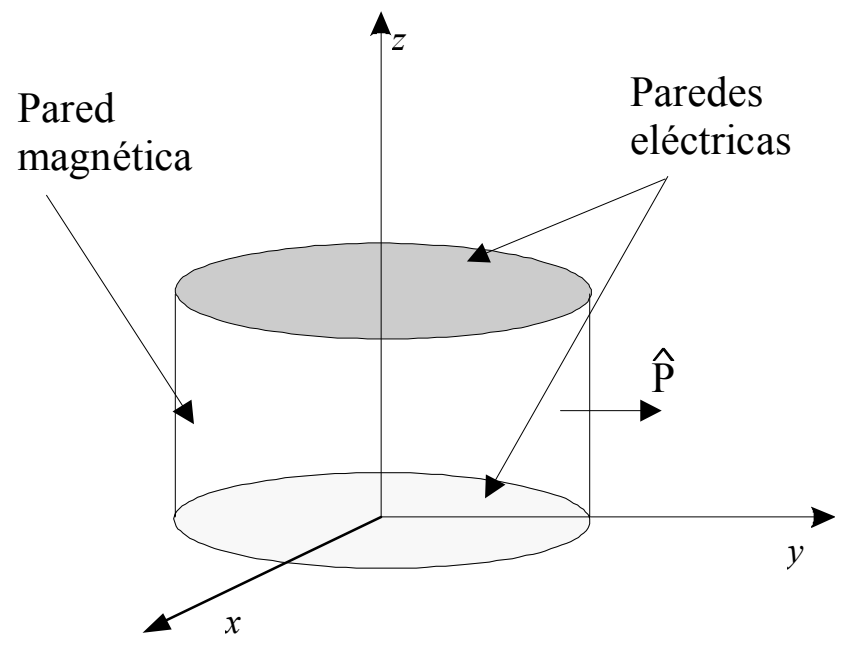

Figura 4.4: Cavidad equivalente de la antena impresa.

Evidentemente, una cavidad de tales características no radiaría, ya que su impedancia de entrada es puramente reactiva e igual a cero o infinito a la frecuencia de resonancia. Sin embargo se puede considerar que la estructura de los campos en la antena es básicamente la misma que en la cavidad equivalente, con lo que se pueden calcular los diagramas de radiación, la potencia radiada total y la impedancia de entrada en cualquier punto del parche. Los resultados que se obtienen son muy parecidos a las medidas. Este método presenta además la ventaja de poder aplicarse a antenas de distintas geometrías.

Para ello se parte de las siguientes observaciones, referidas a la región delimitada por el parche de la Figura 4.5:

๑ Por la proximidad entre el parche y el plano de masa, el campo eléctrico $\vec{E}$ tendrá sólo componente en $\mathrm{z}$, mientras que el campo magnético $\overrightarrow{\mathrm{H}}$ tendrá únicamente componentes en $\mathrm{x}$ e $\mathrm{y}$.

- Los campos son independientes de $\mathrm{z}$ para el rango de frecuencias de interés.

$\infty$ La corriente en el parche no tiene componente normal al borde en ningún punto del mismo, por lo que la componente tangencial de $\overrightarrow{\mathrm{H}}$ a lo largo del perímetro es despreciable. 


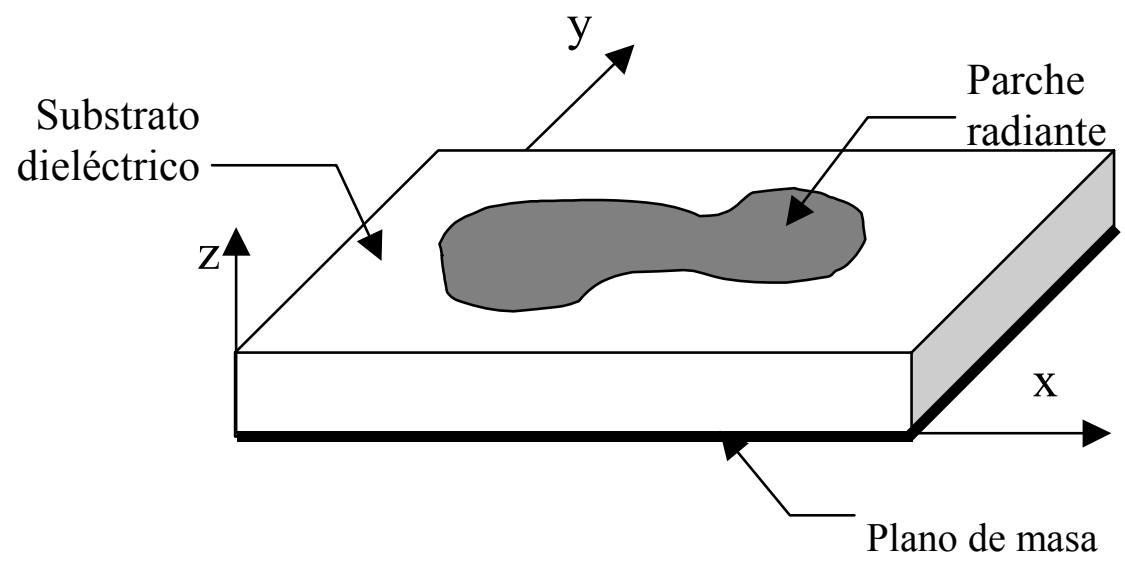

Figura 4.5: Estructura de la antena impresa.

Tenemos entonces para el modelo de cavidad descrito:

$$
\begin{gathered}
\overrightarrow{\mathrm{E}}_{\mathrm{m}}=\psi_{\mathrm{m}} \hat{\mathrm{z}} \quad \overrightarrow{\mathrm{H}}_{\mathrm{m}}=\hat{\mathrm{z}} \times \frac{\nabla_{\mathrm{t}} \psi_{\mathrm{m}}}{\mathrm{j} \omega \mu} \\
\left(\nabla_{\mathrm{t}}^{2}+\mathrm{k}_{\mathrm{m}}{ }^{2}\right) \psi_{\mathrm{m}}=0
\end{gathered}
$$

Con $\frac{\partial \psi_{\mathrm{m}}}{\partial \mathrm{n}}=0$ en la pared magnética.

En estas expresiones, $\psi_{\mathrm{m}}$ representa las variaciones modales, $\hat{\mathrm{z}}$ el vector unitario normal al plano del parche, $\nabla_{\mathrm{t}}$ la parte transversal del operador $\nabla, \hat{\mathrm{n}}$ el vector normal a la pared magnética y $\mathrm{k}_{\mathrm{m}}$ los números de onda correspondientes a las frecuencias de resonancia. Las parejas $\left\{\mathrm{k}_{\mathrm{m}}, \psi_{\mathrm{m}}\right\}$ son las soluciones de la ecuación (4.9). Para un modo $\mathrm{TM}_{\mathrm{mn}}$ :

$$
\mathrm{k}_{\mathrm{m}}=\omega_{\mathrm{mn}} \sqrt{\mu \varepsilon}
$$

Una vez conocida la distribución de campos, se puede aplicar el principio de Huygens a la pared magnética de la cavidad, $\mathrm{C}$, para definir una fuente de corriente magnética en el perímetro:

$$
\overrightarrow{\mathrm{M}}=2 \hat{\mathrm{n}} \times \hat{\mathrm{z}} \mathrm{E}_{\mathrm{z}}
$$

El factor 2 se introduce para tener en cuenta el efecto del plano de masa.

Las corrientes correspondientes se muestran en la Figura 4.6 [4.1] para diferentes modos de un parche rectangular alimentado por una sonda coaxial.

Con (4.11) se puede calcular el vector potencial eléctrico como:

$$
\overrightarrow{\mathrm{F}}(\overrightarrow{\mathrm{r}})=\varepsilon_{0} \int_{C} \frac{\overrightarrow{\mathrm{M}}(\overrightarrow{\mathrm{r}})}{4 \pi\left(\overrightarrow{\mathrm{r}}-\overrightarrow{\mathrm{r}}^{\prime}\right)} \mathrm{e}^{-\mathrm{jk} \mathrm{k}_{0}\left|\overrightarrow{\mathrm{r}}-\overrightarrow{\mathrm{r}}^{\prime}\right|} \mathrm{dl}\left(\overrightarrow{\mathrm{r}}^{\prime}\right)
$$

Los campos lejanos y la potencia radiada total son:

$$
E_{\theta}=\eta H_{\phi}=j k_{0}\left(-F_{x} \sin \phi+F_{y} \cos \phi\right)
$$




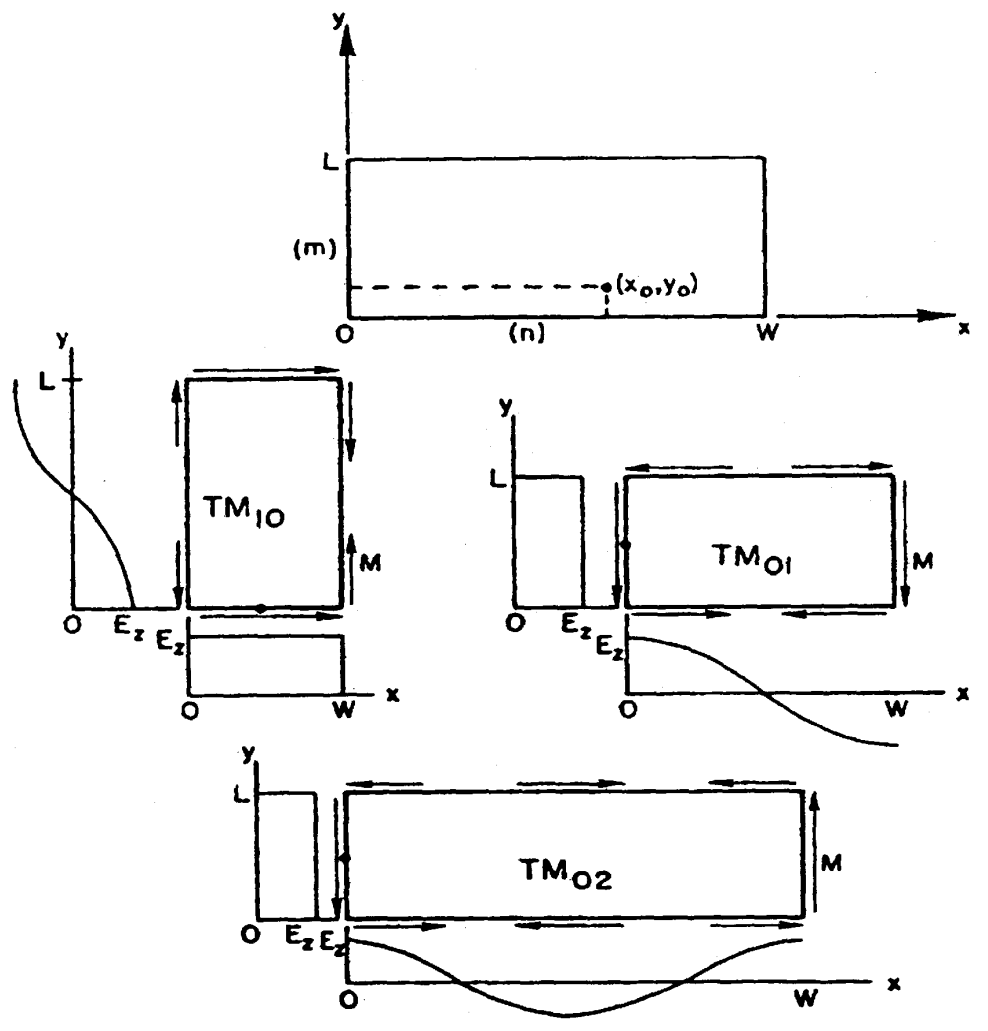

Figura 4.6: Campo eléctrico y distribuciones superficiales de corriente magnética en un parche rectangular.

$$
\begin{gathered}
\mathrm{E}_{\phi}=-\eta \mathrm{H}_{\theta}=\mathrm{jk}_{0}\left(\mathrm{~F}_{\mathrm{x}} \cos \theta \cos \phi+\mathrm{F}_{\mathrm{y}} \sin \theta \sin \phi\right) \\
\mathrm{P}_{\mathrm{rad}}=\int_{0}^{\pi / 2} \int_{0}^{2 \pi}\left(\mathrm{E}_{\theta} \mathrm{H}_{\phi}^{*}-\mathrm{E}_{\phi} \mathrm{H}_{\theta}^{*}\right) \mathrm{r}^{2} \sin \theta \mathrm{d} \phi \mathrm{d} \theta
\end{gathered}
$$

En general, la frecuencia de resonancia obtenida aplicando este método, considerando una cavidad sin pérdidas, es ligeramente inferior a la frecuencia de resonancia real. Esto se debe principalmente a que los campos en los bordes radianes se extienden al espacio libre, por lo que el cálculo del número de onda resulta imposible utilizando únicamente la permitividad del dieléctrico. La frecuencia de resonancia de una cavidad decrece al incrementarse las pérdidas totales, que no se han tenido en cuenta hasta el momento.

El efecto de los campos en los bordes se puede tener en cuenta alejando la pared magnética una distancia $\delta 1$ del borde del parche, mientras que el de las pérdidas se incluye utilizando un valor la tangente de pérdidas del dieléctrico mayor que en el caso sin pérdidas, dado por la fórmula:

$$
\tan \delta_{\mathrm{e}}=\frac{\mathrm{P}_{\mathrm{rad}}+\mathrm{P}_{\mathrm{di}}+\mathrm{P}_{\mathrm{cu}}}{\mathrm{P}_{\mathrm{di}}} \tan \delta_{\mathrm{d}}
$$

Donde $\mathrm{P}_{\mathrm{r}}$ es la potencia radiada obtenida con la ecuación (4.15), $\mathrm{P}_{\mathrm{di}} \mathrm{y} \mathrm{P}_{\mathrm{cu}}$ las potencias disipadas en el substrato y en los conductores, respectivamente, y $\delta_{\mathrm{d}}$ la tangente de pérdidas del dieléctrico. $\mathrm{P}_{\mathrm{di}} \mathrm{Y}$ $\mathrm{P}_{\mathrm{cu}}$ se calculan como: 


$$
\mathrm{P}_{\mathrm{cu}}=\mathrm{R}_{\mathrm{s}} \iint_{\mathrm{S}}\left(\overrightarrow{\mathrm{H}} \cdot \overrightarrow{\mathrm{H}}^{*}\right) \mathrm{dS} \quad \mathrm{P}_{\mathrm{di}}=\frac{\omega \varepsilon^{\prime \prime} \delta}{2} \iiint_{\mathrm{V}}|\overrightarrow{\mathrm{E}}|^{2} \mathrm{dV}
$$

Siendo $\mathrm{R}_{\mathrm{s}}$ la parte real de la impedancia superficial, $\mathrm{S}$ y $\mathrm{V}$ el área del parche y el volumen de la cavidad, respectivamente, $\omega$ la frecuencia angular y $\varepsilon$ " la parte imaginaria de la permitividad compleja $\varepsilon$. La frecuencia de resonancia de un parche rectangular se puede calcular entonces como:

$$
\mathrm{f}_{0}=\frac{\mathrm{c}}{2(1+\delta 1) \sqrt{\varepsilon_{\text {eff }}}}
$$

En la ecuación (4.18) c es la velocidad de la luz en el espacio libre, 1 la longitud física del parche, $\varepsilon_{\text {eff }}$ la constante dieléctrica relativa efectiva del substrato y $\delta 1$ la extensión de longitud para tener en cuenta los efectos en los bordes. Para el cálculo de $\varepsilon_{\text {eff }} \mathrm{y} \delta 1$ se utilizan las expresiones dadas por Wheeler [4.5] y Bahl [4.6], que tienen en cuenta la altura h del substrato, y la anchura W del parche:

$$
\begin{gathered}
\varepsilon_{\text {eff }}=\frac{\varepsilon_{\mathrm{r}}+1}{2}+\frac{\varepsilon_{\mathrm{r}}-1}{2}\left(1+\frac{10 \mathrm{~h}}{\mathrm{~W}}\right)^{-1 / 2} \\
\frac{\delta 1}{\mathrm{~h}}=0,412 \frac{\left(\varepsilon_{\mathrm{eff}}+0,3\right)\left(\frac{\mathrm{W}}{\mathrm{h}}+0,264\right)}{\left(\varepsilon_{\text {eff }}+0,258\right)\left(\frac{\mathrm{W}}{\mathrm{h}}+0,8\right)}
\end{gathered}
$$

Para hallar la impedancia de entrada, se debe calcular la potencia disipada en el parche por unidad de tensión en el puerto de entrada. Los campos radiados se obtienen a partir de los campos en el interior de la cavidad. Si se utiliza un método basado en corrientes eléctricas [4.7], se deducen de las corrientes superficiales $\mathrm{J}_{\mathrm{s}}$ en el parche y en las paredes eléctricas, y de las corrientes volúmicas $\mathrm{J}_{\mathrm{v}}$ en el dieléctrico, si éste está presente dentro de la cavidad.

Por el contrario, el método de las aperturas radiantes [4.8] aplica el teorema de equivalencia a una superficie eléctrica cerrada $S$, que encierra en su interior un campo nulo y fuentes magnéticas equivalentes. Con esta aproximación se estima que la radiación se produce sólo en las paredes magnéticas y se desprecia por lo tanto las corrientes de polarización, que contribuyen únicamente a las componentes en $\theta$ del campo eléctrico, a la hora de calcular los campos lejanos. Este método es pues más impreciso que el de las corrientes eléctricas para determinar las características de radiación de la antena.

Existen ecuaciones alternativas para el cálculo de $\varepsilon_{\text {eff }} \mathrm{y} \delta 1$, como las propuestas por Hammerstad [4.9] y Kirschning [4.10]. Sin embargo, la mayoría están orientadas a líneas microtira, y no permiten obtener resultados satisfactorios en el análisis de antenas. Martin [4.11] propuso a su vez unos parámetros para un modelo de cavidad mejorado, adaptado al cálculo de la frecuencia de resonancia de antenas impresas. Para ello utilizaba la expresión desarrollada por Getsinger [4.12] 
para calcular la constante dieléctrica efectiva:

$$
\begin{gathered}
\varepsilon_{\text {eff }}=\varepsilon_{\mathrm{r}}-\frac{\varepsilon_{\mathrm{r}}-\varepsilon_{\text {eff }_{0}}}{1+\mathrm{G}\left(\frac{\mathrm{f}^{2}}{\mathrm{f}_{\mathrm{p}}^{2}}\right) \mathrm{f}} \\
\mathrm{G}=0,6+0,009 \mathrm{Z}_{0 \mathrm{~m}} \quad \mathrm{f}_{\mathrm{p}}=\frac{\mathrm{Z}_{0 \mathrm{~m}}}{2 \mu_{0} \mathrm{~h}}
\end{gathered}
$$

$\mathrm{Z}_{0 \mathrm{~m}}$ representa la impedancia característica de la línea microstrip que forma la antena, $\mu_{0}$ es la permeabilidad del vacío y $G$ un factor determinado empíricamente. En [4.11] se da también una formula alternativa para la extensión de los bordes, obtenida a partir de la ecuación (4.18):

$$
\beta_{\mathrm{s}} \delta 1=0,0003225 \frac{\mathrm{W}}{\mathrm{h}}+\mathrm{C}
$$

Con:

$$
C=\left\{\begin{array}{cc}
0,606+0,128 \ln \left(\frac{\mathrm{h}}{\lambda_{\mathrm{s}}}\right) & \frac{\mathrm{h}}{\lambda_{\mathrm{s}}} \geq 0,009 \\
0 & \text { resto } \\
\beta_{\mathrm{s}}=\frac{2 \pi \sqrt{\varepsilon_{\text {eff }}}}{\lambda_{\mathrm{s}}} &
\end{array}\right.
$$

$\lambda_{\mathrm{s}} \mathrm{y} \beta_{\mathrm{s}}$ son, respectivamente, la longitud de onda y la constante de fase en el substrato, y W el ancho del parche. El autor presenta en [4.11] una comparación con otros métodos para diferentes antenas, con distintos substratos. Los resultados obtenidos utilizando este modelo empírico son muy precisos.

Khellaf [4.13] presenta, por su parte, una expresión sencilla para determinar la resistencia de un parche rectangular de lados a y $b$ :

$$
\begin{gathered}
\mathrm{R}_{\text {in }}=\frac{92 \eta_{0}}{\mathrm{~A} \pi^{5}(1-\mathrm{B})\left(1-\frac{\mathrm{A}}{15}+\frac{\mathrm{A}^{2}}{240}\right)+\frac{\mathrm{B}}{5}\left(2-\frac{\mathrm{A}}{7}+\frac{\mathrm{A}^{2}}{189}\right)} \\
\mathrm{A}=\left(\frac{\pi \mathrm{a}_{\mathrm{e}}}{\lambda_{0}}\right)^{2} \quad \mathrm{~B}=\left(\frac{2 \mathrm{~b}_{\mathrm{e}}}{\lambda_{0}}\right)^{2} \quad \mathrm{a}_{\mathrm{e}}=\mathrm{a}+2 \Delta \mathrm{a} \quad \mathrm{b}_{\mathrm{e}}=\mathrm{b}+2 \Delta \mathrm{b}
\end{gathered}
$$

\subsubsection{Modelo de red multipuertos.}

Una extensión del método de la cavidad es el modelo de red multipuertos propuesto por Benalla y Gupta [4.14], [4.15]. En él los campos electromagnéticos dentro y fuera de la región definida por el 
parche se estudian por separado, asumiendo que el substrato es fino en términos de longitud de onda.

El parche se considera como una red multipuertos bidimensional, cuyos puertos están situados a lo largo de los bordes. Los campos radiados, las ondas de superficie y los efectos de bordes se representan mediante redes equivalentes conectadas a dichos puertos, tal y como se muestra en la Figura 4.7. En un parche rectangular, cada puerto representa una porción de la longitud $\left(\mathrm{L}_{\mathrm{i}}\right)$ o del ancho $\left(\mathrm{W}_{\mathrm{i}}\right)$ del parche, en la que se asume que el campo es uniforme. Para parches con otras geometrías se utilizan las funciones de Green bidimensionales.

Los elementos de la matriz de impedancias que representa los campos en la cavidad se pueden obtener como:

$$
Z_{i j}=\frac{1}{W_{i} W_{j}} \int_{W_{i}} \int_{W_{j}} G\left(x_{i}, y_{i} \mid x_{j}, y_{j}\right) d_{i} d s_{j}
$$

Siendo $\left(\mathrm{x}_{\mathrm{i}}, \mathrm{y}_{\mathrm{i}}\right)$ y $\left(\mathrm{x}_{\mathrm{j}}, \mathrm{y}_{\mathrm{j}}\right)$ las posiciones respectivas de los puertos de anchos $\mathrm{W}_{\mathrm{i}} \mathrm{y} \mathrm{W}$.

Para tener en cuenta las pérdidas en el dieléctrico se utiliza la permitividad compleja $\varepsilon_{\mathrm{r}}$, y se modifica el valor de la tangente de pérdidas para incluir las pérdidas en los conductores.

Utilizando este método se pueden analizar parches de formas complejas, descomponiéndolos, mediante segmentación y desegmentación, en formas elementales con funciones de Green conocidas.

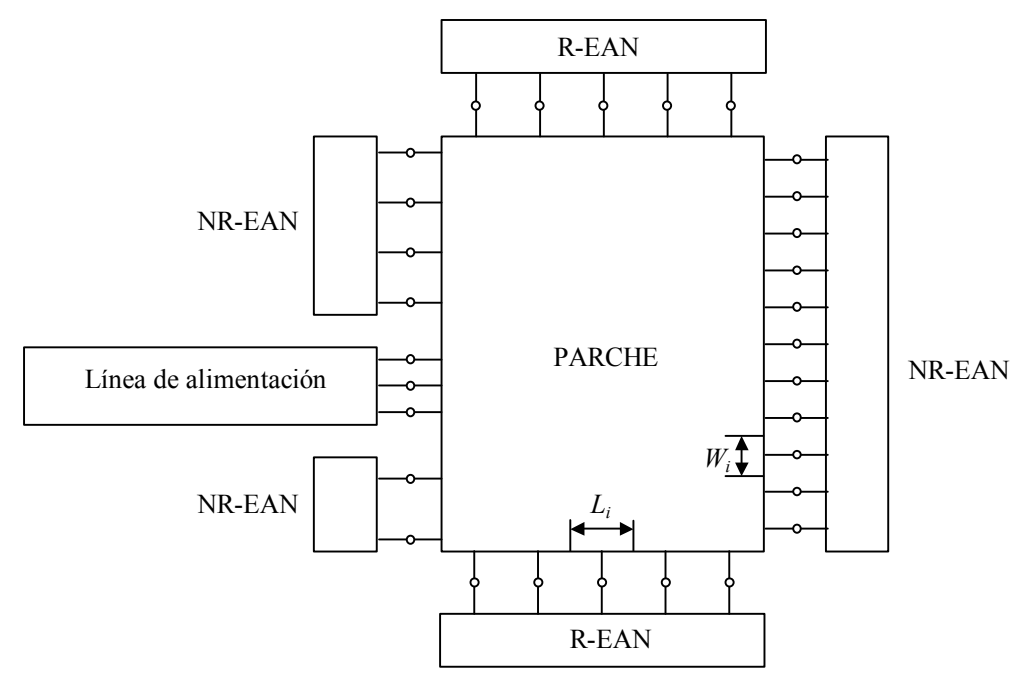

NR-EAN: Red de admitancias equivalente en los bordes no radiantes

R-EAN: Red de admitancias equivalente en los bordes radiantes

Figura 4.7: Modelo de red multipuertos de un parche rectangular.

Para analizar los campos más allá de los bordes del parche, se utilizan redes de admitancias de bordes equivalentes (Edge Admitance Network, EAN), conectadas a los puertos de los lados del parche. Estas redes son una combinación de capacitancias, que representan la energía almacenada en los campos próximos, y conductancias, que simulan el efecto de las ondas de superficie y de los 
campos radiados.

La conductancia se descompone pues en una conductancia de radiación $G_{\mathrm{r}}$ y en una conductancia de ondas de superficie $\mathrm{G}_{\mathrm{s}}$. Los valores de dichas conductancias se obtienen como [4.14]:

$$
\mathrm{G}_{\mathrm{r}}=\frac{2 \mathrm{P}_{\mathrm{rad}}}{\frac{1}{\mathrm{~W}} \int_{0}^{\mathrm{W}} \mathrm{f}^{2}(\mathrm{~s}) \mathrm{ds}} \quad \mathrm{G}_{\mathrm{s}}=\frac{2 \mathrm{P}_{\text {surf }}}{\frac{1}{\mathrm{~W}} \int_{0}^{\mathrm{W}} \mathrm{f}^{2}(\mathrm{~s}) \mathrm{ds}}
$$

Donde $\mathrm{P}_{\mathrm{rad}}$ es la potencia radiada correspondiente a una distribución de tensión uniforme, $\mathrm{f}(\mathrm{s})$ es la distribución de la amplitud de la tensión a lo largo del lado del parche, $\mathrm{W}$ el ancho del parche y $\mathrm{P}_{\text {surf }}$ la potencia asociada a las ondas de superficie. Si el substrato es eléctricamente fino, $\mathrm{G}_{\mathrm{s}}$ puede ser despreciado, ya que será mucho menor que $\mathrm{G}_{\mathrm{r}}$. De forma análoga, existen fórmulas para determinar el valor de las capacitancias de las EANs [4.15].

Es posible igualmente hacer una distinción entre los bordes radiantes y no radiantes a la hora de determinar las matrices de admitancias, R-EAN y NR-EAN respectivamente. En el caso de los bordes no radiantes, es posible a veces simplificar la red equivalente ignorando las conductancias de los bordes, puesto que la radiación de estos bordes es mucho menor que en los bordes radiantes.

Con este modelo se puede tener en cuenta también el efecto de acoplamiento entre las ranuras radiantes equivalentes. Para ello basta con añadir redes de acoplo mutuo (Mutual Coupling Networks, MCN) conectadas a los puertos situados en los bordes radiantes.

Para conectar los campos externos e internos se aplican las leyes de Kirchhoff en los puertos. Resolviendo el sistema se obtiene la impedancia de entrada en el puerto de alimentación, y las tensiones en los puertos de conexión entre la red equivalente del parche y las EANs. Con estos datos se pueden evaluar las características, aplicando el método de segmentación para determinar las tensiones en los bordes radiantes. Estas tensiones se expresan como distribuciones de corriente magnética equivalentes a lo largo de los bordes del parche. Los campos radiados se pueden deducir aplicando la fórmula de Kirchhoff-Huygens.

El caso de antenas cubiertas con superestratos dieléctricos también ha sido considerado[4.16], con resultados que concuerdan con las medidas.

Este modelo presenta varias ventajas respecto al modelo de la cavidad:

- La radiación parásita en el punto de unión entre el parche y la línea de alimentación se puede incorporar al modelo mediante un circuito equivalente conectado al parche por varios puertos, tal y como se muestra en la Figura 4.7. De este modo basta con un sólo puerto a la entrada de la línea de alimentación.

- Se pueden cambiar las fórmulas de cálculo de las admitancias de los bordes para mejorar la precisión del método. Se pueden utilizar también técnicas de optimización y de estudio de sensibilidad, con lo que el método es especialmente adecuado para CAD de antenas impresas. 
\& El acoplamiento entre los bordes radiantes se incluye fácilmente añadiendo una red de acoplo mutuo (MCN). Asimismo se pueden utilizar MCNs para tener en cuenta otros acoplamientos, como entre los bordes no radiantes, entre un borde radiante y otro no radiante, o entre distintos parches dentro de un array.

- Se pueden analizar antenas con más de un punto de alimentación, teniendo en cuenta el acoplamiento entre los puertos.

\subsubsection{Modelo de expansión modal (MEM).}

Este método fue propuesto por Carver en [4.17], al formular ecuaciones de diseño para antenas impresas basadas en una técnica de expansión modal. Aunque este modelo es similar al de la cavidad, se diferencia de él en cuanto que, para llegar a una solución, se imponen condiciones de contorno de impedancia a las cuatro paredes de una cavidad rectangular. Esto implica considerar los efectos la energía radiada y almacenada en los campos próximos en el exterior de la cavidad como admitancias complejas en las paredes. Por otra parte, en el análisis se puede incluir tanto la localización del punto de alimentación como todos los modos excitados, por lo tanto los resultados que se obtienen concuerdan con las medidas.

El parche es tratado como una cavidad resonante delgada de modos $\mathrm{TM}_{\mathrm{mn}}$ transversales a la dirección z, con paredes magnéticas con pérdidas. Se tiene en cuenta en este modelo la variación de los campos a lo largo de los bordes radiantes, así como la posición del punto de alimentación.

El campo eléctrico en la cavidad se desarrolla en serie de Fourier. Como ya se ha dicho, la energía radiada y almacenada en el campo próximo se tienen en cuenta como condiciones de contorno de impedancia. La conductancia de las paredes se equipara a la de una guía de placas paralelas que radia en un el semiespacio por encima del plano de masa, y su susceptancia se basa en la capacitancia del extremo de una línea microtira. Para incluir las pérdidas por radiación y por otros mecanismos se puede modificar la tangente de pérdidas del substrato o las condiciones de contorno en las paredes.

Para un parche rectangular de lados $\mathrm{L}$ y $\mathrm{W}$, con un substrato de permitividad $\varepsilon_{\mathrm{r}} \mathrm{y}$ espesor $\mathrm{h}$ se excitan una serie de modos cuasi-discretos, $\mathrm{TM}_{\mathrm{mn}}$, transversales a la dirección z. Los subíndices $\mathrm{m}$ y n están asociados a las direcciones y y x, respectivamente. El modo dominante, y el que interesa para la radiación, es el $\mathrm{TM}_{10}$, correspondiente a $\mathrm{L}=\lambda_{\mathrm{d}} / 2$, siendo $\lambda_{\mathrm{d}}$ la longitud de onda en el dieléctrico. El campo eléctrico total, asumiendo que el dieléctrico es lo suficientemente fino como para que sólo exista la componente en z, se puede expresar como [4.17]:

$$
\mathrm{E}_{\mathrm{z}}=\sum_{\mathrm{m}} \sum_{\mathrm{n}} \mathrm{A}_{\mathrm{mn}} \mathrm{e}_{\mathrm{mn}}
$$

$A_{m n}$ es el coeficiente de amplitud del modo $T_{m n}, y_{m n}$ el vector de campo eléctrico ortonormalizado en la dirección z. Para una cavidad resonante y no radiante su valor viene dado en [4.17]: 


$$
\mathrm{e}_{\mathrm{mn}}=\frac{\mathrm{X}_{\mathrm{mn}}}{\sqrt{\varepsilon \mathrm{LWh}}} \cos \left(\mathrm{k}_{\mathrm{n}} \mathrm{x}\right) \cos \left(\mathrm{k}_{\mathrm{m}} \mathrm{y}\right)
$$

Del mismo modo, a partir de las ecuaciones de Maxwell se obtiene:

$$
\begin{gathered}
\overrightarrow{\mathrm{h}}_{\mathrm{mn}}=\frac{1}{\mathrm{j} \omega \mu} \frac{\mathrm{X}_{\mathrm{mn}}}{\sqrt{\varepsilon L W h}}\left[\hat{\mathrm{x}} \mathrm{k}_{\mathrm{m}} \cos \left(\mathrm{k}_{\mathrm{n}} \mathrm{x}\right) \sin \left(\mathrm{k}_{\mathrm{m}} \mathrm{y}\right)-\hat{\mathrm{y}} \mathrm{k}_{\mathrm{n}} \sin \left(\mathrm{k}_{\mathrm{n}} \mathrm{x}\right) \cos \left(\mathrm{k}_{\mathrm{m}} \mathrm{y}\right)\right] \\
\left\{\begin{array}{clll}
\mathrm{X}_{00}=1 & (\mathrm{~m}=0 & \mathrm{y} & \mathrm{n}=0) \\
\mathrm{X}_{\mathrm{m} 0}=\mathrm{X}_{0 \mathrm{n}}=\sqrt{2} & (\mathrm{~m}=0 & \mathrm{o} & \mathrm{n}=0) \\
\mathrm{X}_{\mathrm{mn}}=2 & (\mathrm{~m} \neq 0 & \mathrm{y} & \mathrm{n} \neq 0)
\end{array}\right.
\end{gathered}
$$

Los vectores de modo deben satisfacer la ecuación de onda homogénea, y los autovalores la condición de separación:

$$
\mathrm{k}_{\mathrm{mn}}^{2}=\omega_{\mathrm{mn}}^{2} \mu \varepsilon=\mathrm{k}_{\mathrm{n}}^{2}+\mathrm{k}_{\mathrm{m}}^{2}
$$

En el caso de una cavidad no radiante, $\mathrm{k}_{\mathrm{n}}=(\mathrm{n} \pi / \mathrm{L})$ y $\mathrm{k}_{\mathrm{m}}=(\mathrm{m} \pi / \mathrm{W})$. Para que la estructura radie, hay que conectar los campos en el interior de la cavidad con los campos exteriores. Cambiando la condición de contorno homogénea $\hat{\mathrm{n}} \times \overrightarrow{\mathrm{h}}_{\mathrm{mn}}=0$ de la cavidad no radiante por condiciones de contorno de impedancia en las cuatro paredes se obtienen las siguientes ecuaciones trascendentales para los autovalores [4.17]:

$$
\begin{gathered}
\tan \mathrm{k}_{\mathrm{n}} \mathrm{W}=\frac{2 \mathrm{k}_{\mathrm{n}} \alpha_{\mathrm{n}}}{\mathrm{k}_{\mathrm{n}}^{2}-\alpha_{\mathrm{n}}^{2}} \quad \tan \mathrm{k}_{\mathrm{m}} \mathrm{L}=\frac{2 \mathrm{k}_{\mathrm{m}} \alpha_{\mathrm{m}}}{\mathrm{k}_{\mathrm{m}}^{2}-\alpha_{\mathrm{m}}^{2}} \\
\alpha_{\mathrm{n}}=\mathrm{j} \frac{2 \pi \eta_{0}}{\lambda_{0}} \frac{\mathrm{h}}{\mathrm{L}} \mathrm{Y}_{\mathrm{wx}} \mathrm{F}_{\mathrm{x}}(\mathrm{L} / \mathrm{W}) \quad \alpha_{\mathrm{m}}=\mathrm{j} \frac{2 \pi \eta_{0}}{\lambda_{0}} \frac{\mathrm{h}}{\mathrm{W}} \mathrm{Y}_{\mathrm{wy}} \mathrm{F}_{\mathrm{y}}(\mathrm{W} / \mathrm{L})
\end{gathered}
$$

$\mathrm{Y}_{\mathrm{wx}}$ es la admitancia de las paredes en $\mathrm{x}=0 \mathrm{y} \mathrm{x}=\mathrm{W}, \mathrm{Y}_{\mathrm{wy}}$ la admitancia de las paredes en $\mathrm{y}=0$ e $\mathrm{y}=\mathrm{L}$, $\eta_{0}=120 \pi \Omega$ y $\lambda_{0}$ la longitud de onda en el espacio libre. Los factores $F_{\mathrm{x}}$ y $F_{\mathrm{y}}$ se incluyen para tener en cuenta la relación de aspecto de la antena. Para el modo $\mathrm{TM}_{10}, \mathrm{~F}_{\mathrm{x}}=0 \mathrm{y}$ :

$$
\mathrm{F}_{\mathrm{y}}=0,7747+0,5977\left(\frac{\mathrm{W}}{\mathrm{L}}-1\right)-0,1638\left(\frac{\mathrm{W}}{\mathrm{L}}-1\right)^{2}
$$

Para las admitancias de las paredes, $\mathrm{Y}_{\mathrm{w}}$, se pueden utilizar las aproximaciones propuestas por Carver [4.17]:

$$
\begin{gathered}
\mathrm{Y}_{\mathrm{W}}=\mathrm{G}_{\mathrm{W}}+\mathrm{jB} \mathrm{B}_{\mathrm{W}} \\
\mathrm{G}_{\mathrm{W}}=0,00836 \frac{\mathrm{W}}{\lambda_{0}} \\
\mathrm{~B}_{\mathrm{W}}=0,01668 \frac{\delta 1}{\mathrm{~h}} \frac{\mathrm{W}}{\lambda_{0}} \varepsilon_{\text {eff }}
\end{gathered}
$$


$\delta 1$ y $\varepsilon_{\text {eff }}$ se calculan utilizando las fórmulas dadas en las ecuaciones (4.19) y (4.20).

Si la antena está alimentada por un hilo de corriente $\mathrm{I}_{0}$ en la dirección $\mathrm{z}$, de sección rectangular $\left(\mathrm{d}_{\mathrm{x}}, \mathrm{d}_{\mathrm{y}}\right)$ en el punto $\left(\mathrm{x}_{0}, \mathrm{y}_{0}\right)$, los coeficientes de cada modo se pueden calcular con la expresión dada en [4.18]:

$$
\begin{aligned}
A_{m n} & =j I_{0} \sqrt{\frac{\mu h}{W L}} \frac{\mathrm{kX}_{\mathrm{mn}}}{\mathrm{k}^{2}-\mathrm{k}_{\mathrm{mn}}^{2}} \mathrm{G}_{\mathrm{mn}} \cos \left(\mathrm{k}_{\mathrm{n}} \mathrm{x}_{0}\right) \cos \left(\mathrm{k}_{\mathrm{m}} \mathrm{y}_{0}\right) \\
\mathrm{G}_{\mathrm{mn}} & =\frac{\sin \left(\frac{\mathrm{n} \pi \mathrm{d}_{\mathrm{x}}}{2 \mathrm{~W}}\right)}{\frac{\mathrm{n} \pi \mathrm{d}_{\mathrm{x}}}{2 \mathrm{~W}}} \frac{\sin \left(\frac{\mathrm{m} \pi \mathrm{d}_{\mathrm{y}}}{2 \mathrm{~L}}\right)}{\frac{\mathrm{m} \pi \mathrm{d}_{\mathrm{y}}}{2 \mathrm{~L}}} \quad \mathrm{k}_{\mathrm{mn}}=\tilde{\omega}_{\mathrm{mn}} \sqrt{\mu \varepsilon}
\end{aligned}
$$

$\tilde{\omega}_{\mathrm{mn}}$ corresponde a la frecuencia de resonancia compleja del mn-ésimo modo dado por la ecuación (4.34). El factor $\mathrm{G}_{\mathrm{mn}}$ se introduce para tener en cuenta las dimensiones físicas reales del punto de alimentación. Para sondas coaxiales, $\mathrm{d}_{\mathrm{x}}=\mathrm{d}_{\mathrm{y}}$, $\mathrm{y}$ la sección considerada es igual a la sección efectiva de la sonda coaxial. Si la alimentación se hace mediante una línea microtira situada en $\mathrm{y}_{0}=0$, se toma $\mathrm{d}_{\mathrm{y}}=0$, y se considera que el ancho de la línea de alimentación es $\mathrm{d}_{\mathrm{x}}$, obviando los efectos capacitivos que aparecen en el punto de unión.

Con la ecuación (4.34) se puede hallar la frecuencia de resonancia del modo $\mathrm{TM}_{10}$. Para dicho modo, $\mathrm{k}_{\mathrm{n}}=0$ y la frecuencia de resonancia se obtiene como:

$$
\begin{gathered}
\tilde{\omega}=\omega_{\mathrm{r}}+\mathrm{j} \omega_{\mathrm{i}}=\frac{\mathrm{c}}{\sqrt{\varepsilon_{\mathrm{r}}(1-\mathrm{j} \tan \delta)}} \mathrm{k}_{\mathrm{r}} \\
\mathrm{f}_{\mathrm{r}}=\frac{\omega_{\mathrm{r}}}{2 \pi}
\end{gathered}
$$

El autovalor complejo $\mathrm{k}_{10}$ se obtiene aplicando un sencillo algoritmo:

$$
\begin{gathered}
\mathrm{k}_{10}=\left(\frac{\pi}{\mathrm{L}}\right)-\left(\frac{\Delta_{4}}{\mathrm{~L}}\right) \\
\Delta_{0}=0 \quad \Delta_{\mathrm{p}+1}=\frac{2\left(\alpha_{10} \mathrm{~L}\right)\left(\pi-\Delta_{\mathrm{p}}\right)}{\left(\alpha_{10} \mathrm{~L}\right)^{2}+2 \Delta_{\mathrm{p}} \pi-\Delta_{\mathrm{p}}^{2}-\pi^{2}}-\frac{\Delta_{\mathrm{p}}^{3}}{3}
\end{gathered}
$$

Esta expresión corresponde a los dos primeros términos del desarrollo de Taylor en función de $\pi$ de la ecuación (4.35) para el modo $\mathrm{TM}_{10}$. El factor de calidad se puede entonces calcular utilizando las fórmulas dadas en el capítulo anterior.

Si el parche radiante se representa como el circuito RLC paralelo de la Figura 4.8, la impedancia de entrada, el ancho de banda y la eficiencia vienen dados por las ecuaciones (4.47) a (4.49): 


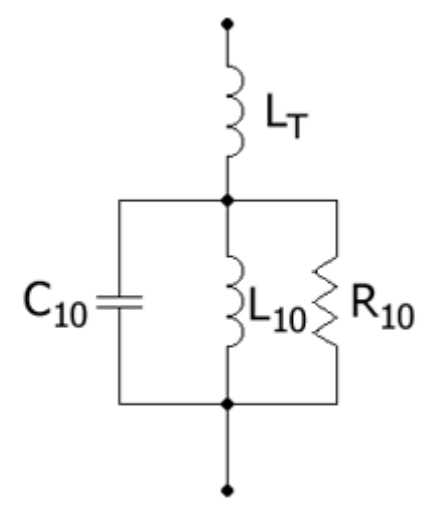

Figura 4.8: Circuito RLC equivalente de una antena impresa.

$$
\begin{gathered}
\mathrm{C}_{10}=\frac{\varepsilon_{\text {eff }} \varepsilon_{\mathrm{o}} \mathrm{LW}}{2 \mathrm{~h}} \cos ^{-2}\left(\frac{\pi \mathrm{y}_{0}}{\mathrm{~L}}\right) \quad \mathrm{L}_{10}=\frac{1}{\mathrm{C}_{1} \omega_{\mathrm{r}}^{2}} \quad \mathrm{R}_{10}=\frac{\mathrm{Q}}{\mathrm{C}_{1} \omega_{\mathrm{r}}} \\
\mathrm{R}_{\mathrm{r}}=\frac{1}{2 \mathrm{C}_{1} \omega_{\mathrm{i}}} \quad \eta=\frac{\mathrm{R}_{\mathrm{r}}}{\mathrm{R}_{1}} \quad \mathrm{BW}=\frac{\mathrm{f}_{\mathrm{r}}}{\mathrm{Q}_{\mathrm{r}}} \\
\mathrm{Z}_{\text {in }}=-\mathrm{j} \frac{\mathrm{h} \omega}{\varepsilon \mathrm{LW}} \sum_{\mathrm{m}=0}^{\infty} \sum_{\mathrm{n}=0}^{\infty} \frac{\varepsilon_{\mathrm{mn}}^{2} \cos ^{2}\left(\frac{\mathrm{n} \pi \mathrm{x}_{0}}{\mathrm{~W}}\right) \cos ^{2}\left(\frac{\mathrm{m} \pi \mathrm{y}_{0}}{\mathrm{~L}}\right)}{\omega^{2}-\tilde{\omega}_{\mathrm{mn}}^{2}} \mathrm{G}_{\mathrm{mn}}
\end{gathered}
$$

La exactitud de este método está limitada por la precisión en la determinación de las impedancias de las paredes. Existen igualmente dificultades si el modo dominante no es excitado con la suficiente fuerza. Sin embargo la desviación entre el modelo y las medidas es mínima. Otra ventaja del método es que proporciona una excelente visión de los mecanismos físicos del funcionamiento de la antena.

\subsubsection{Modelo de línea de transmisión.}

La estructura de las antenas impresas se basa en la de las líneas microtira, por lo tanto es lógico pensar que se pueden analizar como tales. El modelo de la línea de transmisión permite obtener resultados adecuados en la mayoría de los casos, con unos requerimientos de capacidad de cálculo menores que en los métodos descritos anteriormente. Por contra, este método permite analizar únicamente estructuras rectangulares.

La antena se compone de una tira conductora, un substrato y un plano de masa, y es alimentada bien por una línea microtira, bien por una sonda coaxial. La frecuencia de resonancia se determina a partir de la longitud $\mathrm{L}$ y el ancho $\mathrm{W}$ del conductor, y el espesor h y la permitividad $\varepsilon_{\mathrm{r}}$ del substrato. Se asume que tanto el dieléctrico como el plano de masa tienen dimensiones infinitas, por lo que los resultados finales diferirán del comportamiento real del parche. Para compensarlo, es posible utilizar un método asintótico para predecir el diagrama de radiación en el plano E para antenas con un plano de masa finito. Para ello se utiliza el modelo de la cavidad, con una corriente magnética a lo largo de los bordes del parche, y se aplica el principio de Huygens. Sin embargo 
este método no puede ser utilizado para estudiar la radiación para ángulos cercanos a $\theta=90^{\circ}$.

El parche, representado en la Figura 4.9, se equipara a dos ranuras paralelas situadas en la posición de los bordes radiantes del parche. Cada una de ellas tiene una longitud W, y radia en el semiespacio por encima del plano de masa. La admitancia de la ranura se calcula como [4.18]:

$$
\begin{gathered}
\mathrm{G}_{1}+\mathrm{j} \mathrm{B}_{1}=\frac{\pi \mathrm{W}}{\lambda_{0} \mathrm{Z}_{0}}\left\{1+\mathrm{j}\left[1-0,636 \ln \left(\mathrm{k}_{0} \mathrm{w}\right)\right]\right\} \\
\mathrm{Z}_{0}=\sqrt{\frac{\mu_{0}}{\varepsilon_{0}}} \quad \mathrm{k}_{0}=\frac{2 \pi}{\lambda_{0}}
\end{gathered}
$$

En estas expresiones, $\lambda_{0}$ es la longitud de onda en el espacio libre, y w el ancho de la ranura, con $\mathrm{W} \approx \mathrm{h}$.
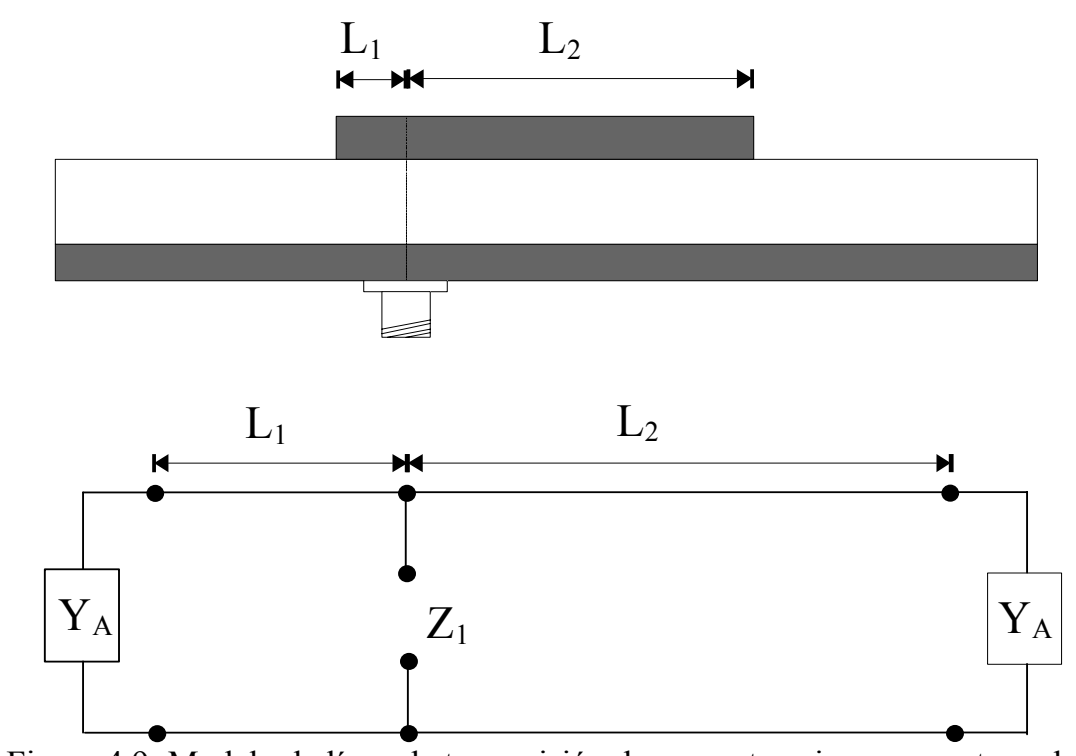

Figura 4.9: Modelo de línea de transmisión de una antena impresa rectangular.

La admitancia de ambos bordes radiantes se calcula con la misma fórmula, ya que ambas ranuras equivalentes son idénticas, si no se tiene en cuenta el efecto de los campos evanescentes cerca del punto de alimentación. Si se asume además que el campo no varía en la dirección paralela al borde radiante, la admitancia característica de la línea microtira viene dada por:

$$
\mathrm{Y}_{0}=\frac{\mathrm{W} \sqrt{\varepsilon_{\mathrm{r}}}}{\mathrm{hZ}}
$$

Para que la excitación de cada ranura esté en oposición de fase, la distancia $L$ entre ambas se fija en algo menos de $\lambda_{\mathrm{d}} / 2$ (típicamente entre $0,48 \lambda_{\mathrm{d}}$ y $0,49 \lambda_{\mathrm{d}}$ ), con $\lambda_{\mathrm{d}}=\lambda_{0} / \sqrt{\varepsilon_{\mathrm{r}}}$, para compensar el efecto de los campos en los bordes. A la frecuencia de resonancia, la admitancia de entrada y la frecuencia de resonancia son entonces:

$$
\mathrm{Y}_{\text {in }}=2 \mathrm{G}_{1}
$$




$$
\mathrm{f}_{\mathrm{r}}=\frac{\mathrm{c}}{\lambda_{\mathrm{d}} \sqrt{\varepsilon_{\mathrm{r}}}}
$$

La principal ventaja de este método reside en su sencillez, puesto que la frecuencia de resonancia y la resistencia de entrada se pueden calcular utilizando fórmulas simples. Sin embargo, esto conlleva ciertas inexactitudes.

En el modelo original de línea de transmisión se proponía una fórmula simple para determinar la admitancia en paralelo, $\mathrm{Y}_{\mathrm{s}}$ :

$$
\begin{gathered}
Y_{s}=W y_{s} \\
y_{s}=g_{s}+j b_{s}
\end{gathered}
$$

$\mathrm{y}_{\mathrm{s}}$ representa la admitancia por unidad de longitud de una ranura de longitud infinita y anchura $\mathrm{w}$ excitada de forma uniforme sobre un plano conductor perfecto de dimensiones infinitas.

La representación de los bordes en circuito abierto por la admitancia en paralelo $\mathrm{Y}_{\mathrm{s}}$ incluye los efectos de radiación y de las ondas de superficie, en la parte real $\mathrm{G}_{\mathrm{s}}$, y la energía almacenada en los campos próximos que se extienden más allá del final de la tira conductora, en la parte imaginaria $B_{s}$. Esto significa que la exactitud del método depende en gran medida de la elección de $G_{s}$ y $B_{s}$. Sin embargo las expresiones propuestas en el modelo original no son sino aproximaciones que desprecian el efecto del acoplamiento mutuo entre las ranuras equivalentes y la radiación en las paredes laterales.

En este modelo no se tienen en cuenta tampoco específicamente las ondas de superficie, por lo que sólo se puede aplicar a antenas con dieléctrico fino. Se ignoran asimismo las variaciones de campo a lo largo de los bordes radiantes y las corrientes transversales a la longitud del parche.

Existen igualmente modelos de línea de transmisión para antenas con dos puertos, útiles para el diseño de arrays lineales o planos alimentados en serie [4.19]. Se han considerado también los casos en que la alimentación se hace a través de los lados radiantes o no radiantes [4.20].

Antes de que Pues propusiera un modelo mejorado, diversos autores sugirieron métodos para solventar los problemas anteriormente citados. Derneryd [4.21] aportó una corrección de la parte real de $\mathrm{Y}_{\mathrm{s}}$, considerando las terminaciones en circuito abierto como una red RC, de cuya expresión integral Lier [4.22] derivó una expresión analítica aproximada.

En lo referente a las paredes laterales, Hammer [4.8] desprecia su efecto al calcular los campos radiados, utilizando un valor aproximado constante $\mathrm{E}_{\mathrm{a}}$ para la componente tangencial del campo eléctrico en el plano $\mathrm{z}=0$ sobre una distancia al borde del parche $2 \mathrm{a}$, con $\mathrm{a}<<1$, y considerando $\left|\mathrm{E}_{\mathrm{t}}\right|=0$ más allá de este límite. Las limitaciones impuestas a las antenas impresas por esta radiación del borde en circuito abierto y por la que aparece en las transiciones del punto de alimentación son consideradas en detalle por James [4.23]. Chang [4.24] por su parte considera el caso de una onda 
TEM que incide oblicuamente desde el interior de un parche semi-infinito, para proporcionar una visión de diversos mecanismos de radiación y dispersión que se producen en una antena impresa.

Para superar las limitaciones del modelo de línea de transmisión original, Pues [4.25] propone un modelo mejorado, como el que se muestra en la Figura 4.10.

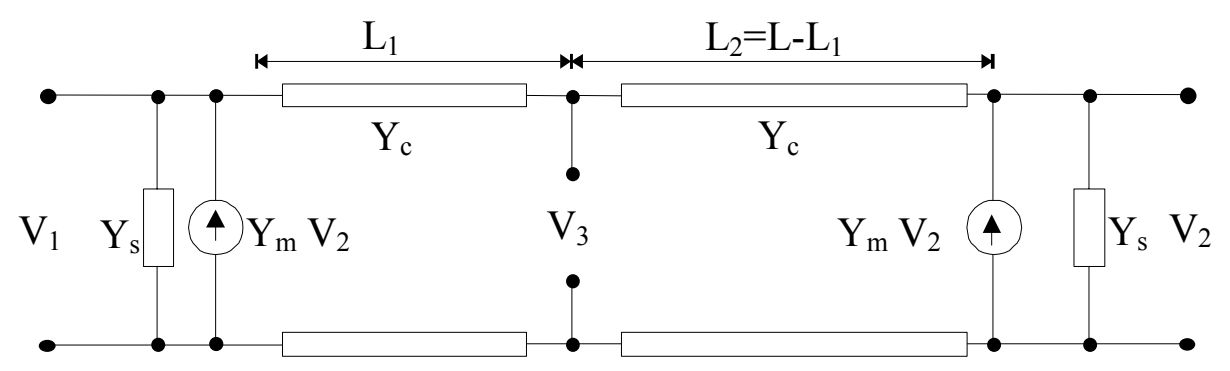

Figura 4.10: Modelo de línea de transmisión mejorado.

En este modelo, $Y_{\mathrm{s}}$ representa la autoadmitancia de los extremos en circuito abierto del parche, e $\mathrm{Y}_{\mathrm{m}}$ su admitancia de radiación mutua. El efecto del acoplamiento mutuo entre las ranuras radiantes equivalentes se considera por medio de generadores de corriente controlados por tensión. Las pérdidas en el dieléctrico y en los conductores se incluyen en la constante de atenuación $\alpha$, que es la parte real de la constante de propagación compleja $\gamma$.

Para simular el efecto de la línea de alimentación sobre el comportamiento de la antena, la admitancia $Y_{s}$ situada frente a ella se puede reducir un factor $r$, lo que equivaldría a añadir una admitancia en paralelo $\mathrm{Y}_{\mathrm{F}}$.

Los parámetros de este modelo se presentan en forma de expresiones cerradas, por lo que pueden ser programadas con relativa facilidad y utilizadas en combinación con programas de CAD para líneas microtira.

La expresión de la admitancia de entrada de la antena alimentada por línea microtira es, en este caso:

$$
Y_{\text {in }}=\frac{Y_{c}^{2}+Y_{s}^{2}-Y_{m}^{2}+2 Y_{s} Y_{c} \operatorname{coth}\left(\gamma_{p} L\right)-2 Y_{m} Y_{c} \operatorname{csch}\left(\gamma_{p} L\right)}{Y_{s}+Y_{c} \operatorname{coth}\left(\gamma_{p} L\right)}
$$

Donde $\operatorname{coth}(z)$ y $\operatorname{csch}(z)$ representan respectivamente las funciones cotangente y cosecante hiperbólicas complejas de argumento $z$.

Este método proporciona resultados precisos, obtenidos con una buena eficiencia de cálculo. Tiene además un amplio rango de validez en lo referente a la relación de aspecto del parche W/L, la constante dieléctrica $\varepsilon_{\mathrm{r}} \mathrm{y}$ del grosor eléctrico $\mathrm{h} / \lambda_{0}$ del substrato. Por contra, no se tienen en cuenta efectos como la propagación de ondas de superficie, el acoplamiento mutuo entre diferentes parches, la difracción en los bordes del sustrato y del plano de masa o los modos de orden superior. 


\subsection{MÉTODOS NUMÉRICOS.}

Los modelos analíticos descritos en la sección anterior sirven para una primera aproximación a las características de las antenas, pero su uso se limita a elementos de características muy específicas. Algunas de las limitaciones son las siguientes:

Q El modelo de cavidad con una distribución de corriente uniforme en el punto de alimentación sólo es válido cuando el grosor del substrato es mucho menor que la longitud de onda (inferior a $0,01 \lambda_{0}$ ).

\& En el modelo de expansión modal se consideran también substratos eléctricamente finos, y la precisión de los resultados obtenidos depende de la excitación de un modo dominante lo suficientemente fuerte, así como de la exactitud de las fórmulas utilizadas para las admitancias de pared.

\& Para el modelo de línea de transmisión el substrato tiene dimensiones infinitas, y se ignoran las variaciones de campo a lo largo de los bordes radiantes, así como la aparición de ondas de superficie. La exactitud del modelo se limita a espesores de substrato menores que $0,02 \lambda_{0}$.

Si además se deben tener en cuenta otros parámetros, como acoplamientos mutuos o la impedancia de la línea de alimentación, la complejidad de los modelos aumenta considerablemente y se recurre a simplificaciones y aproximaciones. Sin embargo, estos modelos simplificados pueden llevar a errores, sobre todo para el cálculo de impedancias de entrada.

Por otra parte, los modelos analíticos son válidos únicamente en un determinado rango de frecuencias, y para elementos radiantes de formas simples. No se pueden considerar con exactitud antenas de forma arbitraria, o con elementos parásitos.

Si a esto se añade que, debido a su estrecho ancho de banda, el diseño de antenas impresas debe ser llevado a cabo con una gran precisión, tanto desde el punto de vista eléctrico como mecánico, resulta hoy en día imprescindible disponer de técnicas numéricas de alta eficiencia. Así, los simuladores 2,5-D y 3-D basados en ellas resultan un elemento clave para el desarrollo de elementos radiantes de formas arbitrarias. Dichos simuladores permiten obtener resultados de gran precisión, puesto que pretenden resolver las ecuaciones de onda de forma rigurosa, realizando pocas aproximaciones. De esta forma resulta posible analizar estructuras radiantes de forma arbitraria, para las cuales cualquier predicción respecto a la distribución de corrientes resulta extremadamente compleja, cuando no imposible. Es además posible estudiar, entre otros, los efectos producidos por el uso de substratos gruesos, acoplamientos mutuos entre diferentes parches, o la presencia de la red de alimentación.

Para el análisis de antenas impresas hay que considerar tres factores principales:

La existencia de, al menos, dos dieléctricos: el aire y uno o más substratos. 
- La existencia de condiciones de contorno no homogéneas, puesto que las superficies metálicas utilizadas como elementos radiantes se encuentran en los interfaces entre diferentes medios dieléctricos.

- La naturaleza finita del plano de masa, que en determinados casos puede ser despreciada, asumiendo que sus bordes se encuentran lejos de la estructura, en términos de longitud de onda. Se considera entonces en los cálculos que el plano de masa se extiende hasta el infinito.

En este apartado se describen algunos de los métodos numéricos más comunes.

\subsubsection{Ecuación integral de potencial mixta (MPIE).}

El Modelo de la Ecuación Integral de Potencial Mixta (Mixed Potential Integral Equation, MPIE) [4.26] es la base de numerosos simuladores electromagnéticos 2,5D. En él se tienen en cuenta efectos como el acoplamiento mutuo entre elementos de un array, la existencia de ondas de superficie o las pérdidas en conductores y dieléctricos. Es posible utilizarlo para el análisis de elementos de geometría arbitraria, sin limitación en la frecuencia de trabajo o el grosor de la estructura.

En este modelo, se asume que una antena microtira es un caso particular de medio estratificado. Así, el parche se representa mediante una ecuación cuya principal incógnita es la distribución superficial de corriente eléctrica. Las funciones de Green necesarias se obtienen como transformadas inversas de Hankel, aplicando la teoría de los medios estratificados. La ecuación integral en el dominio espacial se compone de un potencial vector y un potencial escalar.

En el análisis utilizando el MPIE se consideran los campos de excitación $\overrightarrow{\mathrm{E}}^{\mathrm{e}}$ y $\overrightarrow{\mathrm{H}}^{\mathrm{e}}$ como, o bien ondas planas originadas en el infinito, o bien campos creados por una fuente finita situada en el espacio delimitado por la antena. Estos campos inducen corrientes en la superficie superior del plano de masa y en ambas caras del parche. Si se considera que el grosor del parche metálico es nulo, se puede sustituir por una lámina de corriente $\mathrm{J}_{\mathrm{S}}$, igual a la suma algebraica de las corrientes en las superficies superior e inferior, $\mathrm{J}_{\mathrm{S} 1}$ y $\mathrm{J}_{\mathrm{S} 2}$, tal y como se muestra en la Figura 4.11. Estas distribuciones de corriente generan a su vez campos difractados $\overrightarrow{\mathrm{E}}^{\mathrm{d}}$ y $\overrightarrow{\mathrm{H}}^{\mathrm{d}}$, que, junto con los campos de excitación, dan lugar a los campos totales.

Las condiciones de contorno del problema son inhomogéneas, debido a la discontinuidad de la componente tangencial del campo magnético difractado $\overrightarrow{\mathrm{H}}^{\mathrm{d}}$. Esta discontinuidad es la que da lugar a las corrientes superficiales en las superficies conductoras [4.27]. Si se consideran las pérdidas en los conductores, el campo eléctrico tangencial total está relacionado con la densidad superficial de corriente como (condición de Leontovitch): 


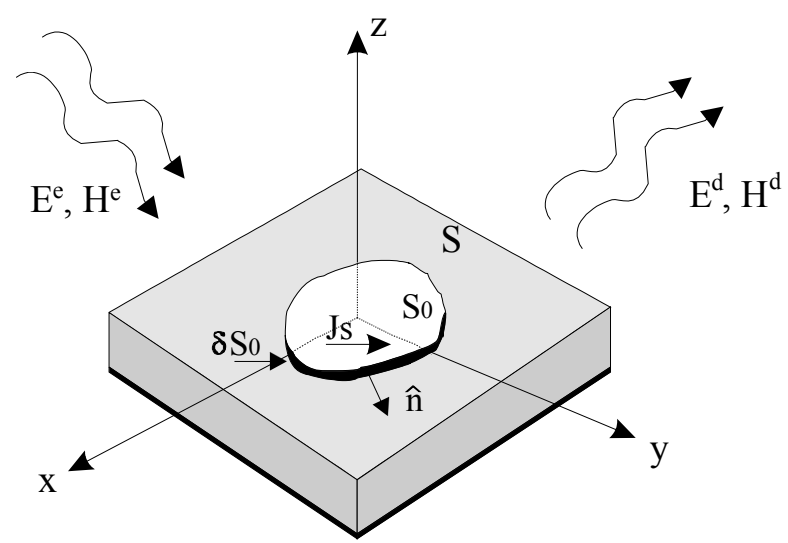

Figura 4.11: Modelo equivalente del parche.

$$
\overrightarrow{\mathrm{e}}_{\mathrm{z}} \times\left(\overrightarrow{\mathrm{E}}^{\mathrm{e}}+\overrightarrow{\mathrm{E}}^{\mathrm{d}}\right)=\overrightarrow{\mathrm{e}}_{\mathrm{z}} \times \mathrm{Z}_{\mathrm{S}} \overrightarrow{\mathrm{J}}_{\mathrm{S}}
$$

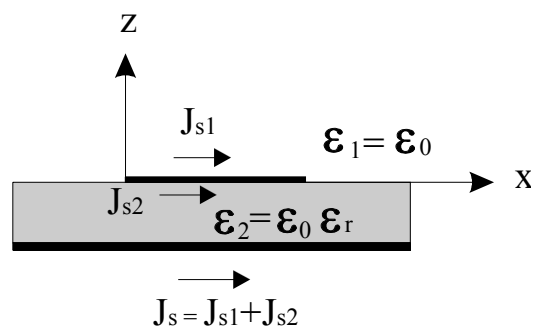

Siendo $Z_{S}$ la impedancia superficial del parche conductor, que incluye el efecto de la conductividad finita de la superficie metálica. Su valor se debe obtener a partir de medidas. Sin embargo, si el espesor del parche es mucho mayor que la profundidad del efecto pelicular (skin depth) $\delta, Z_{\mathrm{S}}$ se puede aproximar por:

$$
Z_{s}=\frac{(1+j)}{\sigma^{*} \delta}
$$

En esta expresión, $\sigma^{*}$ representa la conductividad efectiva, que puede ser mucho menor que la nominal.

Con la condición de contorno inhomogénea definida por la ecuación (4.58), las ecuaciones de Maxwell para condiciones de contorno homogéneas, las ecuaciones de Helmholtz para los potenciales y la condición de Lorentz se obtiene la forma final de la ecuación integral:

$$
\overrightarrow{\mathrm{e}}_{\mathrm{z}} \times\left(j \omega \int_{\mathrm{S}_{0}} \overline{\overline{\mathrm{G}_{\mathrm{A}}}} \overrightarrow{\mathrm{J}}_{\mathrm{S}} \mathrm{dS}+\nabla \int_{\mathrm{S}_{0}} \mathrm{G}_{\mathrm{V}} \mathrm{q}_{\mathrm{S}} \mathrm{d} \mathrm{S}^{\prime}+\mathrm{Z}_{\mathrm{S}} \overrightarrow{\mathrm{J}}_{\mathrm{S}}\right)=\overrightarrow{\mathrm{e}}_{\mathrm{z}} \times \overrightarrow{\mathrm{E}}^{\mathrm{e}}
$$

En ella, $S_{0}$ representa la superficie del parche, $\overline{\overline{G_{A}}}$ es una función de Green diádica, $G_{v}$ la función de Green asociada al potencial escalar y $\mathrm{q}_{\mathrm{s}}$ la densidad de carga asociada a la superficie. $\vec{J}_{\mathrm{S}}$ y $\mathrm{q}_{\mathrm{S}}$ son las incógnitas de esta ecuación integral, y están relacionadas mediante la ecuación de continuidad:

$$
\nabla \overrightarrow{\mathrm{J}}_{\mathrm{S}}+\mathrm{j} \omega \mathrm{q}_{\mathrm{S}}=0
$$

La ecuación integral (4.60) es una ecuación integral de Fredholm de segunda especie [4.28]. La exactitud de su resolución depende de la exactitud de los algoritmos utilizados para evaluar las funciones de Green $G_{v}$ y $\overline{\overline{G_{A}}}$. Para la resolución del problema, se empieza por calcular las funciones de Green. Las distribuciones superficiales de corriente $\overrightarrow{\mathrm{J}}_{\mathrm{S}}$, incógnitas del problema, se desarrollan utilizando un conjunto de funciones base. La ecuación integral se compara con un conjunto de funciones de test utilizando un método numérico adecuado, como el Método de los 
Momentos, que se describe más adelante. Se transforma así la ecuación integral en un conjunto de ecuaciones lineales, cuyos autovalores complejos permiten obtener las frecuencias de resonancia del parche no perturbado, y su factor de calidad en ausencia de cargas.

Si la integral se resuelve en forma de presentada en la ecuación (4.60), se considera que se ha utilizado un método en el dominio temporal. Si primero se aplica un atransformada Fourier, el método pertenece al dominio espectral.

Una descripción detallada de la ecuación integral para antenas microtira se puede encontrar en [4.29].

\subsubsection{Método de momentos (MoM).}

En los métodos numéricos en el dominio temporal las funciones de Green se representan mediante una integral de Sommerfeld. Sin embargo, ésta presenta una singularidad en el origen y problemas de convergencia. Es necesario pues recurrir a representaciones asintóticas.

Por contra, se dispone de expresiones cerradas de las funciones de Green en el dominio espacial. Sin embargo, no es posible extender la integral hasta el infinito, y si la distancia lateral es grande pueden aparecer oscilaciones rápidas del integrando.

Se ha podido comprobar [4.30] que se obtiene una mayor eficiencia en el cálculo si se combinan los métodos en el dominio espacial y el dominio temporal. Para ello, aunque existen alternativas como la técnica de Ajuste de Modos (Mode-Matching) [4.31] o el Análisis en el Dominio Espectral (Spectral Domain Analysis, SDA) [4.32], el Método de los Momentos (Method of Moments, MoM) [4.33] es tal vez el más extendido.

Tal y como se ha mencionado anteriormente, es necesario discretizar la ecuación integral (4.60) para transformarla en una ecuación matricial. Una forma de lograrlo es aplicar el Método de los Momentos, propuesto por Harrington en los años 60 [4.34]. Para ello se desarrolla la corriente superficial en una serie de funciones base. Dicho desarrollo (discretización) se limita a un número finito de términos. Para determinar los coeficientes de las funciones base, la ecuación integral se multiplica por unas funciones de test predefinidas, y se integra, para obtener un sistema de ecuaciones lineales. Este sistema de ecuaciones se puede entonces resolver utilizando diferentes métodos numéricos. Con este método es posible realizar el análisis de antenas de geometría arbitraria, así como de estructuras multicapa.

E1 MoM puede descomponerse en tres pasos [4.35]:

1. Definición de un conjunto de ecuaciones base y desarrollo de la función incógnita de la ecuación integral en una serie de funciones base.

Así, una ecuación integral continua se puede representar como: 


$$
\mathrm{L}(\mathrm{J})=\mathrm{B}
$$

Donde $\mathrm{J}$ es la incógnita, por ejemplo la distribución superficial de corrientes. B representa la excitación, que puede ser igual a cero, y L es un operador lineal, por ejemplo un operador diferencial o integral.

Para aproximar la solución de la ecuación integral, $\mathrm{J}$ se desarrolla en una serie de $\mathrm{N}$ funciones base adecuadas:

$$
\mathrm{J}=\sum_{\mathrm{n}=1}^{\mathrm{N}} \mathrm{C}_{\mathrm{n}} \mathrm{I}_{\mathrm{n}}
$$

Donde $C_{n}$ representa el coeficiente por determinar de la función base $I_{n}$. Al sustituir (4.63) en la ecuación integral se produce un error residual $\varepsilon_{\mathrm{R}}$ :

$$
\varepsilon_{R}=\sum_{n=1}^{N} C_{n} L\left(I_{n}\right)-B
$$

Puesto que el operador utilizado es lineal, se puede alterar el orden de los términos para introducirlo dentro del sumatorio.

\section{Construcción del producto interno para ambos lados de la ecuación integral y definición del} sistema de ecuaciones lineal.

Al definir un conjunto de funciones de peso $\mathrm{W}_{\mathrm{m}}$ para el operador $\mathrm{L}$ y construir el producto interno se deriva la siguiente expresión:

$$
<\mathrm{W}_{\mathrm{m}}, \varepsilon_{\mathrm{R}}>=\sum_{\mathrm{n}=1}^{\mathrm{N}} \mathrm{C}_{\mathrm{n}}<\mathrm{W}_{\mathrm{m}}, \mathrm{L}\left(\mathrm{I}_{\mathrm{n}}\right)>-<\mathrm{W}_{\mathrm{m}}, \mathrm{B}>\quad \mathrm{m}=1, . ., \mathrm{N}
$$

Con ello se obtiene un sistema de $\mathrm{N}$ ecuaciones con $\mathrm{N}$ incógnitas, los coeficientes $\mathrm{C}_{\mathrm{n}}$. El producto interno está definido por:

$$
<\mathrm{P}, \mathrm{Q}>=\iint_{\mathrm{S}} \mathrm{P} \cdot \mathrm{QdS} \rightarrow \iint_{\mathrm{S}} \mathrm{W}_{\mathrm{m}} \cdot \varepsilon_{\mathrm{R}} \mathrm{dS}=\sum_{\mathrm{n}=1}^{\mathrm{N}} \mathrm{C}_{\mathrm{n}} \iint_{\mathrm{S}} \mathrm{W}_{\mathrm{m}} \cdot \mathrm{L}\left(\mathrm{I}_{\mathrm{n}}\right) \mathrm{dS}-\iint_{\mathrm{S}} \mathrm{W}_{\mathrm{m}} \cdot \mathrm{BdS}
$$

$\mathrm{S}$ se extiende a todo el dominio de definición de las funciones $\mathrm{P}$ y $\mathrm{Q}, \mathrm{y}$ puede representar, por ejemplo, la superficie de un cuerpo radiante. Si en la ecuación (4.65) se considera que el error residual es ortogonal a las $\mathrm{N}$ funciones peso, se obtiene un sistema de ecuaciones lineales de la forma:

$$
\vec{Z} \cdot \vec{X}=\vec{Y}
$$

Los elementos de la matriz $m \times n \vec{Z}$, a menudo denominada matriz de impedancias, y el vector de excitación $\overrightarrow{\mathrm{Y}}$ vienen dados por: 


$$
\begin{gathered}
\overrightarrow{\mathrm{Z}}_{\mathrm{mn}}=<\mathrm{W}_{\mathrm{m}}, \mathrm{L}\left(\mathrm{I}_{\mathrm{n}}\right)>=\iint_{\mathrm{S}} \mathrm{W}_{\mathrm{m}} \cdot \mathrm{L}\left(\mathrm{I}_{\mathrm{n}}\right) \mathrm{dS} \\
\overrightarrow{\mathrm{Y}}_{\mathrm{m}}=<\mathrm{W}_{\mathrm{m}}, \mathrm{B}>=\iint_{\mathrm{S}} \mathrm{W}_{\mathrm{m}} \cdot \mathrm{BdS}
\end{gathered}
$$

El vector $\vec{X}$ recoge los coeficientes $C_{n}$, aún por determinar. La ecuación integral ha sido pues reducida a un sistema de ecuaciones lineales, homogéneo o inhomogéneo.

\section{Resolución del sistema.}

Debido a la definición del sistema de ecuaciones, para el cálculo de los elementos de la matriz de impedancias es necesario llevar a cabo una integración doble: primero sobre las funciones de base correspondientes al operador L, y posteriormente sobre las funciones de test. Dichas integraciones se suelen llevar a cabo de forma numérica. Una vez determinados los elementos de las matrices, se debe resolver el sistema de ecuaciones. Para ello se pueden utilizar diferentes métodos numéricos, como por ejemplo el método de eliminación de Gauss, el método de Seidel, el método de máxima pendiente o el del gradiente conjugado.

El procedimiento descrito hasta aquí se puede denominar MoM directo, ya que la solución se obtiene a partir de un número determinado de operaciones matemáticas elementales. Existe otra variante, conocida como MoM iterativo o indirecto. En ella se intenta determinar el mínimo de la función de error residual $\varepsilon_{\mathrm{R}}$ mediante un proceso de búsqueda iterativo. El MoM indirecto requiere métodos matemáticos más complejos, pero permite reducir el espacio de memoria necesario para la resolución de un problema.

Existen además dos variantes del MoM: en el dominio espacial y en el dominio espectral. La primera consiste en resolver la ecuación integral en la que las incógnitas son la distribución de corrientes en la superficie del parche conductor y la corriente de alimentación. Para ello se asume en primer lugar que no existe dieléctrico, y se aplica la teoría de las imágenes, para eliminar el plano de masa. La alimentación se modela mediante un hilo de corriente. La corriente en la superficie del parche, $\mathrm{J}_{\mathrm{s}}$, se desarrolla sobre un conjunto de $\mathrm{N}$ funciones $\mathrm{J}_{\mathrm{n}}$. La ecuación integral se analiza considerando $\mathrm{N}$ fuentes eléctricas de test situadas en la superficie $\mathrm{S}$ que delimita la antena. Se obtiene así un sistema de $\mathrm{N}$ ecuaciones lineales simultáneas, cuyos coeficientes corresponden a una matriz de impedancias $Z_{\mathrm{mn}}$. Si el dieléctrico no es aire, su efecto se tiene en cuenta utilizando el teorema de equivalencia de volumen, y añadiendo un incremento $\Delta Z_{\mathrm{mn}}$ a la matriz de impedancias $\mathrm{Z}_{\mathrm{mn}}$.

Para la aplicación de este método al estudio de parches radiantes hay que tener en cuenta diversos factores:

- La corriente superficial $\mathrm{J}_{\mathrm{S}}$ corresponde a las caras internas del parche y del plano de masa, pero no a la corriente en la cara externa del parche, y no permite por lo tanto calcular directamente los campos en el exterior. 
E El MoM no proporciona ninguna conexión directa entre los campos en el interior y el exterior de la antena.

- Para la evaluación de las integrales para construir la matriz de impedancias y determinar las fuentes vectoriales de tensión (source voltage vector elements) hay que tener en cuenta la geometría y la polarización de cada parche.

En [4.36] se extiende este método al análisis de antenas impresas de forma arbitraria sobre un substrato dieléctrico. También se han llevado a cabo estudios sobre parches apilados (stacked patches), utilizando la condición de Leontovitch y aplicando la MPIE a la superficie de cada parche [4.30].

Para poder aplicar el método con éxito se debe dividir la estructura que se pretende analizar en celdas elementales, en las cuales se puede realizar una aproximación sencilla de la distribución superficial de corriente. Para ello se recurre normalmente a celdas rectangulares y triangulares. Las primeras requieren cálculos menos complejos, y suelen ser suficientes para la mayoría de los casos. En el caso de celdas rectangulares, a la hora de implementar las funciones base el contorno del parche se suele aproximar por una línea poligonal tipo "Manhattan", tal y como se describe en la Figura 4.12.

La mayor parte del tiempo necesario para la resolución del problema no se dedica al cálculo de los elementos de la matriz de impedancias, sino a su inversión para resolver el sistema de ecuaciones. Si un modelo consta de M segmentos lineales, y en cada uno de ellos la corriente se aproxima por una serie de $\mathrm{N}$ funciones base, para determinar los coeficientes de la matriz de impedancias se requiere calcular numéricamente $2 \mathrm{xMxN}$ integrales, y resolver un sistema de $\mathrm{MxN}$ ecuaciones lineales. Si se considera que normalmente se requiere un mínimo de 10 segmentos de mallado por longitud de onda (100 segmentos en un cuadrado de lado $\lambda$ ) y unas tres funciones base por segmento para obtener resultados exactos, se debe resolver un sistema de 300 ecuaciones lineales por cuadrado de lado $\lambda$, con otras tantas incógnitas. Si el tamaño del modelo de simulación es mucho mayor que la longitud de onda, el número de ecuaciones crece rápidamente, prolongando el tiempo de simulación y dificultando la resolución del problema.

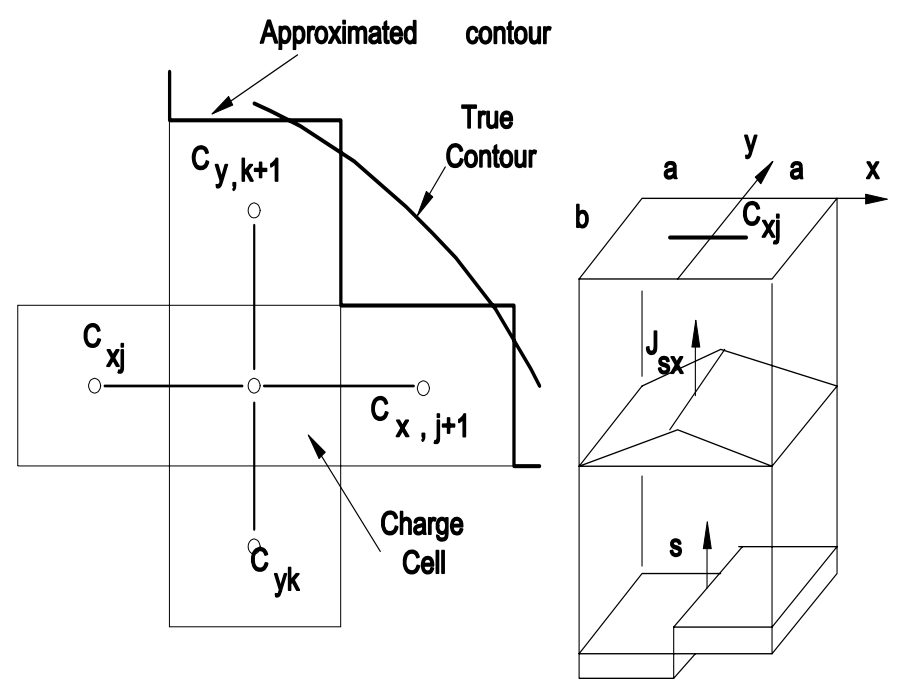

Figura 4.12: Descomposición del parche en los bordes. 
La elección de las funciones base influye también en la complejidad numérica de la resolución del sistema lineal. Ciertas funciones permiten calcular analíticamente las integrales para obtener los elementos de la matriz de impedancias. Así, las funciones base y las funciones de test determinan en parte el tamaño del sistema de ecuaciones. La elección de dichas funciones debe ser sopesada detenidamente. En el método de Galerkin, por ejemplo, se toman las mismas funciones como funciones de base y de test.

Las funciones de base se pueden dividir en dos categorías: entire domain functions (funciones de dominio completo) y sub-domain functions (funciones de subdominio) [4.35]. Entre las funciones que se suelen utilizar destacan las funciones de Dirac, de Heavyside, pulsos, funciones trigonométricas o wavelets.

La desventaja del MoM en el dominio espacial es la necesidad de una evaluación numérica de la matriz extremadamente precisa, puesto que de lo contrario resulta mal condicionada y difícil de invertir. Esto da lugar a un incremento significativo del tiempo computacional requerido. Una variante de esta técnica es el MoM en el dominio espectral que no necesita tanto tiempo de computación pero sufre de una pérdida de la visión intuitiva del problema al transformarlo al dominio espectral.

\subsubsection{Diferencias finitas en el dominio temporal (FDTD).}

El método de las Diferencias Finitas en el Dominio Temporal (Finite-Differences, Time-Domain, FDTD) supone la forma más directa de acceder a los campos calculados numéricamente. Este método se basa en una discretización de las ecuaciones de Maxwell en forma diferencial, conforme a una malla determinada. El principio fue desarrollado por Yee en los años 60 [4.37]. Está basado en una discretización tanto espacial como temporal de las ecuaciones de Maxwell en forma diferencial:

$$
\begin{gathered}
\nabla \times \overrightarrow{\mathrm{H}}=\overrightarrow{\mathrm{J}}+\frac{\partial \overrightarrow{\mathrm{D}}}{\partial \mathrm{t}} \\
\nabla \times \overrightarrow{\mathrm{E}}=-\frac{\partial \overrightarrow{\mathrm{B}}}{\partial \mathrm{t}}
\end{gathered}
$$

Si se desarrollan las ecuaciones (4.72) y (4.73) en un sistema de coordenadas cartesianas se obtiene un sistema con una ecuación para cada una de las componentes de los campos eléctrico y magnético $\left(E_{x}, E_{y}, E_{z}, H_{x}, H_{y}, H_{z}\right)$. Para simplificar, se supone $\vec{J}=0$. Se obtiene así un sistema de seis ecuaciones con seis componentes de campo incógnitas, que puede ser resuelto de forma iterativa. Al discretizar este sistema se obtienen las siguientes expresiones [4.38]: 


$$
\begin{gathered}
\frac{\partial \mathrm{H}_{\mathrm{x}}(\mathrm{x}, \mathrm{y}, \mathrm{z})}{\partial \mathrm{t}}=\frac{1}{\mu_{\mathrm{x}}}\left(\frac{\partial \mathrm{E}_{\mathrm{y}}(\mathrm{x}, \mathrm{y}, \mathrm{z})}{\partial \mathrm{z}}-\frac{\partial \mathrm{E}_{\mathrm{z}}(\mathrm{x}, \mathrm{y}, \mathrm{z})}{\partial \mathrm{y}}\right) \\
\Downarrow \\
=\frac{1}{\mu_{\mathrm{x}}}\left[\frac{\mathrm{E}_{\mathrm{y}}^{\mathrm{n}}\left(\mathrm{x}, \mathrm{y}+\frac{\Delta \mathrm{y}}{2}, \mathrm{z}\right)-\mathrm{E}_{\mathrm{y}}^{\mathrm{n}}\left(\mathrm{x}, \mathrm{y}-\frac{\Delta \mathrm{y}}{2}, \mathrm{z}\right)}{\Delta \mathrm{z}}-\frac{\mathrm{E}_{\mathrm{z}}^{\mathrm{n}+\frac{1}{2}}\left(\mathrm{x}, \mathrm{y}, \mathrm{z}+\frac{\Delta \mathrm{z}}{2}\right)-\mathrm{E}_{\mathrm{z}}^{\mathrm{n}}\left(\mathrm{x}, \mathrm{y}, \mathrm{z}-\frac{\left.\Delta \mathrm{z})-\mathrm{H}_{\mathrm{x}}^{\mathrm{n}-\frac{1}{2}}\right)}{\Delta}\right)}{\Delta \mathrm{y}, \mathrm{y}, \mathrm{z})}=\right.
\end{gathered}
$$

En la ecuación (4.73) n denota el número del paso que se está calculando en el momento actual $\Delta t$. $\Delta \mathrm{x}, \Delta \mathrm{y}$ y $\Delta \mathrm{z}$ resultan de la discretización espacial. Así, se puede ver que la derivada temporal del campo eléctrico se obtiene a partir de la derivada espacial del campo magnético. Para facilitar la comprensión, Yee ideó una representación gráfica del problema, la celda de Yee, que se muestra en la Figura 4.13. En ella, cada componente de campo eléctrico está rodeada por cuatro componentes de campo magnético, y viceversa.

Con este sistema, si se calcula el campo eléctrico en el instante $\mathrm{n} \cdot \Delta \mathrm{t}$ y el campo magnético en el instante $(\mathrm{n}+1 / 2) \cdot \Delta \mathrm{t}$, se obtiene una aproximación de la derivada temporal mediante el método de las diferencias centrales (central differences). De este modo, cada nuevo valor de una componente de campo depende únicamente de su valor anterior y del valor anterior del resto de las componentes de campo en unos puntos determinados de la estructura (procedimiento leapfrog). Así, el método FDTD calcula alternativamente el campo eléctrico y el campo magnético.

Para resolver totalmente las ecuaciones de Maxwell, se debe conocer la distribución de campos en cada instante, así como los límites del espacio de simulación. Por lo tanto, al inicio de la simulación, el espacio considerado se supone vacío de energía, y la estructura considerada será excitada por una fuente determinada.

A continuación se describen brevemente las diferentes etapas de la simulación.

\section{Discretización de la estructura.}

La estructura que se quiere simular debe ser dividida en celdas como la definida por Yee. Hay que crear por lo tanto un mallado tridimensional que contenga la totalidad de la estructura.

Para evitar que el cálculo sea numéricamente inestable debido a distancias espaciales demasiado grandes, se debe respetar el siguiente criterio de estabilidad [4.38]:

$$
\mathrm{c} \cdot \Delta \mathrm{t} \leq \frac{1}{\sqrt{\frac{1}{\Delta \mathrm{x}^{2}}+\frac{1}{\Delta \mathrm{y}^{2}}+\frac{1}{\Delta \mathrm{z}^{2}}}}
$$




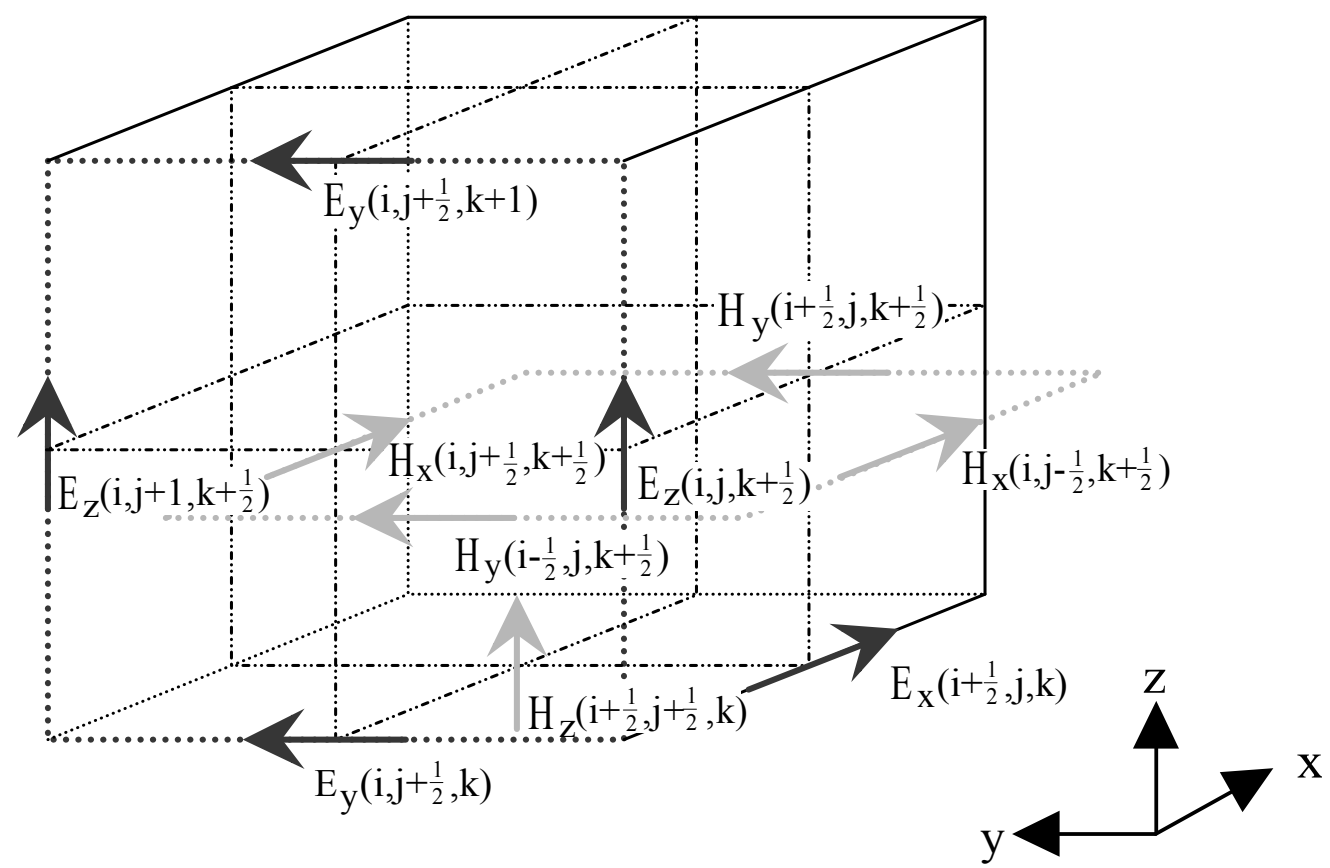

Figura 4.13: Distribución de las componentes de campo en la celda de Yee $(i=x, j=y, k=z)$.

Donde $\mathrm{c}$ denota la velocidad de la luz en el vacío. El paso temporal $\Delta \mathrm{t}$ lo determina normalmente el programa de simulación a partir de la discretización espacial $(\Delta \mathrm{x}, \Delta \mathrm{y}, \Delta \mathrm{z})$ definida por el usuario.

Hay que tener en cuenta además la dispersión numérica en la propagación de las ondas, que también depende del tamaño de la discretización. Este problema es despreciable si se toma un tamaño de celda inferior a 1/20 de la longitud de onda en cada dirección del espacio.

Respetando estas limitaciones, es posible también definir mallas no uniformes, para ajustarse a las necesidades de la estructura.

\section{Definición de las condiciones de contorno.}

En los métodos numéricos que operan en el dominio temporal es siempre necesario definir los límites del espacio en el que se va a llevar a cabo la simulación (computational box). La elección de las condiciones de contorno depende de la estructura considerada. En el caso de las antenas, se utilizan normalmente paredes absorbentes para simular condiciones de espacio libre. Hay que garantizar que las ondas electromagnéticas no sean reflejadas en las paredes, ya que afectaría a la exactitud de los resultados.

Existen diferentes tipos de paredes absorbentes, como por ejemplo las de Mur [4.39], o las Capas Perfectamente Adaptadas (Perfectly Matched Layers, PML) [4.40]. Éstas últimas son las más utilizadas en la resolución de problemas de antenas.

\section{Definición de la excitación.}

Lo siguiente que hay que definir es un puerto para la excitación de la estructura. Por este puerto se 
introducirá la señal de excitación temporal, que suele ser un pulso gaussiano. Este pulso presenta la ventaja de tener un espectro en frecuencia también gaussiano, y proporciona información desde 0 $\mathrm{Hz}$ hasta la frecuencia de corte deseada ajustando el ancho del pulso. Esta señal se propaga desde el puerto de entrada por toda la estructura, y es difractado y reflejado en las discontinuidades, como aristas o interfaces entre diferentes materiales. La evaluación de la respuesta temporal se puede efectuar en el mismo puerto de entrada (reflexión), o se pueden definir más puertos en la estructura, para acceder a la señal temporal (transmisión).

\section{Simulación.}

El simulador calcula, para cada instante de tiempo, la distribución del pulso introducido en la estructura. Así, éste se propaga paulatinamente por toda la estructura.

\section{Transformada de Fourier.}

Una vez que la señal recuperada en el puerto de salida se ha atenuado lo suficiente, hasta alcanzar el denominado estado estacionario, se puede dar por finalizada la simulación. Se procede entonces a aplicar una transformada de Fourier a los resultados obtenidos en el dominio temporal. Se obtienen así los resultados deseados en el dominio espectral: coeficiente de reflexión, de transmisión, impedancia, campos lejanos, etc.

La FDTD permite analizar un gran número de estructuras complejas, tanto planas como tridimensionales. La ventaja del método reside en el hecho de que, una vez obtenidos los resultados en el dominio temporal, la transformada de Fourier permite obtener datos en el dominio espectral sobre un amplio ancho de banda que se puede determinar a partir de la forma del pulso de excitación. Así, no es necesario reiniciar una nueva simulación para obtener los datos a una determinada frecuencia.

La principal desventaja del método reside en el elevado tiempo de simulación. Cuanto mayor sea la estructura considerada, mayor será el número de puntos en los que se deberán calcular los campos a cada paso, aumentando así el tiempo de simulación, incluso si se utiliza un mallado no uniforme. Otro factor determinante para la duración del proceso es el tipo de estructura considerado. Así, si se tiene una estructura altamente resonante, el pulso de excitación puede tardar mucho en decaer, alargando así el tiempo total de simulación.

\subsubsection{Matriz de Líneas de Transmisión (TLM).}

Con el método de la Matriz de Líneas de Transmisión (Transmission Line Matrix, TLM) se pretende resolver el problema discretizando tanto el espacio como el tiempo [4.41]. Con ello se logra estudiar correctamente cierto tipo de antenas como parche apilados (stacked) con plano de masa de dimensiones finitas. 
El método utiliza los denominados nodos condensados simétricos (symmetrical condensed node), propuestos por Johns [4.42], y se basa en el principio de Huygens [4.43].

La estructura que se pretende simular se divide en celdas elementales mediante un proceso de discretización. En el centro de cada celda se encuentra un nodo, y en cada cara de la celda se definen dos puertos asociados a dicho nodo. Estos puertos permiten la conexión con las celdas vecinas. Cada nodo tiene asociados 12 puertos, cuya distribución se muestra en la Figura 4.14. A lo largo de las conexiones se propagan determinados pulsos, que alcanzan así el nodo y se propagan hacia los nodos vecinos, donde el proceso se repite. Este proceso se reproduce cíclicamente durante un determinado espacio de tiempo $\Delta \mathrm{t}$.

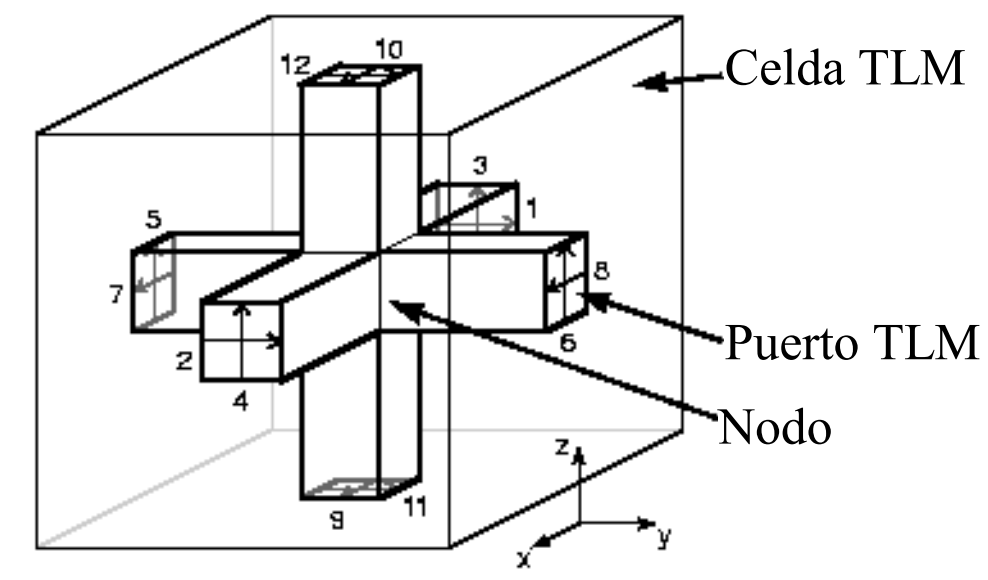

Figura 4.14: Estructura de un nodo y numeración de los puertos en una celda TLM.

El proceso que se lleva a cabo en cada nodo se puede escribir como:

$$
\overrightarrow{\mathrm{S}}_{\mathrm{i}, \mathrm{j}, \mathrm{k}}^{\mathrm{n}} \cdot \overrightarrow{\mathrm{P}}_{\mathrm{i}, \mathrm{j}, \mathrm{k}}^{\mathrm{n}}=\overrightarrow{\mathrm{Q}}_{\mathrm{i}, \mathrm{j}, \mathrm{k}}^{\mathrm{n}}
$$

Donde $\overrightarrow{\mathrm{S}}_{\mathrm{i}, \mathrm{j}, \mathrm{k}}^{\mathrm{n}}$ representa la matriz de dispersión de la celda $(\mathrm{i}, \mathrm{j}, \mathrm{k})$. Los índices (i,j,k) denotan la posición de la celda en el sistema de coordenadas tridimensional $(\mathrm{x}, \mathrm{y}, \mathrm{z})$. El vector $\overrightarrow{\mathrm{P}}_{\mathrm{i}, \mathrm{j}, \mathrm{k}}^{\mathrm{n}}$ define los pulsos TLM incidentes en el instante $n$. Al llegar al nodo en el centro de la celda son rerradiados hacia las celdas vecinas. Estos pulsos salientes, $\overrightarrow{\mathrm{Q}}_{\mathrm{i}, \mathrm{j}, \mathrm{k}}^{\mathrm{n}}$, inciden sobre las celdas vecinas, donde se repite el proceso. Así, el pulso entrante en una celda en el instante $n+1$ es igual a los pulsos salientes de sus celdas vecinas en el instante n. Esta operación se lleva a cabo simultáneamente en todas las celdas de la estructura.

En el caso de estructuras abiertas, hay que tener en cuenta la radiación en el espacio exterior a la zona mallada. Esto se logra con la utilización de paredes absorbentes, como las utilizadas con la FDTD. Además, la relación entre el tamaño del mallado $\Delta \mathrm{l}$, mostrado en la Figura 4.15 , y el paso temporal es fijo, y no se puede alterar [4.44]. Por lo tanto, un mallado no uniforme es sólo posible manipulando las propiedades de la malla TLM. 


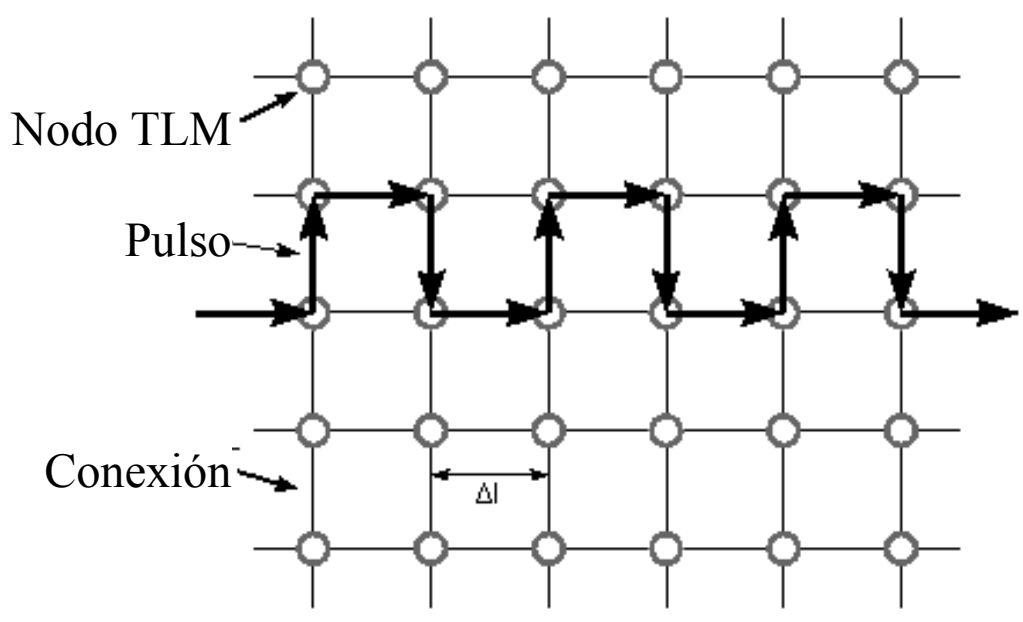

Figura 4.15: Propagación de un pulso en una malla TLM bidimensional (corte 2D de una malla tridimensional).

Las simulaciones utilizando el método TLM requieren una gran capacidad de almacenamiento, ya que para la representación de las seis componentes de campo presentes en una celda TLM son necesarios doce pulsos TLM.

El método TLM permite simular la propagación de ondas electromagnéticas en un medio cualquiera. Las paredes absorbentes deben tener una alta calidad, y absorber las ondas incidentes para un amplio rango de ángulos de incidencia para lograr resultados precisos sobre una gran banda de frecuencias. Para obtener los campos lejanos es necesario llevar a cabo una transformación campo cercano-campo lejano en el dominio temporal. Sin embargo, para obtener la impedancia de entrada de una antena se requiere un análisis en el dominio espectral.

\subsection{SIMULADORES ELECTROMAGNÉTICOS COMERCIALES.}

\subsubsection{Características de los simuladores.}

Hoy en día resulta imposible concebir el diseño de antenas para comunicaciones sin recurrir para ello a programas de simulación. Dos aspectos fundamentales determinaron esta tendencia: por una parte, el incremento exponencial de la capacidad de cálculo de los ordenadores, y por otra la tendencia a la miniaturización y a la integración de los elementos radiantes. Así pues, las herramientas de simulación han dejado de limitarse al análisis de estructuras, y se usan hoy en día también para su diseño y síntesis.

Debido a esta evolución, en el mercado se encuentran un gran número de simuladores con diferentes utilidades: sencillos programas para aproximar las características de antenas elementales o agrupaciones, métodos numéricos de aplicación general, programas específicos para antenas 
planas, etc. Estos programas se distinguen no sólo por los algoritmos implementados, sino también en lo que respecta a los datos de entrada y salida, a la utilización de los recursos del sistema y al tiempo de cálculo.

Para el diseño de antenas es imprescindible disponer de herramientas de simulación electromagnética $(\mathrm{em})$ potentes, ya sean de desarrollo propio, o productos comerciales. Para ello existe la posibilidad de desarrollar algoritmos especialmente adaptados al problema que se desea resolver. Éstos permiten obtener una gran eficiencia de cálculo para esa aplicación específica, pero por contra dificultan la introducción de cambios en la geometría o las características de la estructura.

La utilización de simuladores em comerciales está justificada en gran medida por su flexibilidad [4.45]. Sin embargo, es necesario determinar cuál es el método numérico que mejor se adapta a cada situación y cuál permite analizar con mayor eficiencia cada estructura. Así, se suelen preferir los simuladores em 3-D de onda completa, que permiten analizar estructuras de forma arbitraria. Sin embargo, hay que tener en cuenta que el paso más costoso en tiempo es la resolución de la matriz $\left(\mathrm{O}\left(\mathrm{n}^{3}\right)\right.$, donde $\mathrm{n}$ es el número de incógnitas), mientras que su construcción depende de $\mathrm{n}$ como $\mathrm{O}\left(\mathrm{n}^{2}\right)$. Para antenas de forma compleja, en las que es necesario una discretización muy fina de la estructura, el uso de simuladores 3-D puede llevar a problemas cuyo tiempo de resolución es excesivamente largo.

A menudo suele bastar con utilizar simuladores em que pueden simular estructuras con un número ilimitado de capas metálicas y dieléctricas planas. Para estos casos, en los que se tienen corrientes en 2-D pero campos en 3-D, se ha acuñado el término de simuladores 2,5-D [4.46]. Con ellos no es posible analizar estructuras con dieléctricos que no son planos o de dimensiones infinitas. Sin embargo, si únicamente se consideran diferentes capas horizontales de dieléctricos planos, y se permite el uso de corrientes verticales que modelan los conectores coaxiales o las vías, se utiliza preferentemente la denominación de simuladores em 3-D planos.

Los simuladores 3-D planos suelen resultar útiles en las primeras etapas de diseño de antenas impresas, en las que la utilización de simuladores 3-D completos sería demasiado costosa en términos de tiempo. Así, el uso de simuladores 2,5-D y 3-D planos permite agilizar el análisis de estructuras con condiciones especiales, como planos de masa infinitos con una o más capas, también infinitas, de material dieléctrico. Estos resultados preliminares resultan útiles para un posterior análisis más exhaustivo de la estructura, utilizando simuladores 3-D completos.

En la Tabla 4.1 se recogen las características de los simuladores em comerciales disponibles en la actualidad. 
Tabla 4.1: Características de algunos simuladores em.

\begin{tabular}{|c|c|c|c|c|c|c|c|c|}
\hline Nombre & Algoritmo. & Tipo & Pérdidas & $\begin{array}{c}\text { Metal + } \\
\text { dieléctr.. }\end{array}$ & $\begin{array}{c}\text { Plano masa } \\
\text { finito }\end{array}$ & $\begin{array}{c}\text { Dieléctrico } \\
\text { finito }\end{array}$ & Estruct.3D & Discretización \\
\hline $\begin{array}{c}\text { IE3D [4.47] } \\
\text { Zeland Softw. }\end{array}$ & MoM & $\begin{array}{c}3 \mathrm{D} \text { (metal) } \\
2,5 \mathrm{D} \text { (dieléctr.) }\end{array}$ & Sí & Sí & Sí & No & Sí (Metal) & Automática \\
\hline $\begin{array}{c}\text { Micro-Stripes } \\
{[4.48]} \\
\text { Kimberley } \\
\text { Communic. C.. }\end{array}$ & TLM & $3 \mathrm{D}$ & Sí & Sí & Sí & Sí & Sí & $\begin{array}{c}\text { Semi- } \\
\text { automática }\end{array}$ \\
\hline $\begin{array}{c}\text { EMPIRE [4.49] } \\
\text { IMST GmbH }\end{array}$ & FDTD & $3 \mathrm{D}$ & Sí & Sí & Sí & Sí & Sí & $\begin{array}{c}\text { Semi- } \\
\text { automática }\end{array}$ \\
\hline $\begin{array}{c}\text { Concept [4.50] } \\
\text { Uni. Hamburg- } \\
\text { Harburg }\end{array}$ & MoM & $3 \mathrm{D}$ & Sí & $\begin{array}{l}\text { Sí (con } \\
\text { límites) }\end{array}$ & Sí & Sí & Sí & Manual \\
\hline $\begin{array}{c}\text { Ensemble [4.51] } \\
\text { Ansoft Co. }\end{array}$ & MoM & $2,5 \mathrm{D}$ & Sí & Sí & Sí & No & No & Automática \\
\hline $\begin{array}{l}\text { XFDTD [4.52] } \\
\text { Remcom Inc. }\end{array}$ & FDTD & $3 \mathrm{D}$ & Sí & Sí & Sí & Sí & Sí & $\begin{array}{c}\text { Semi- } \\
\text { automática }\end{array}$ \\
\hline $\begin{array}{c}\text { HFSS [4.51] } \\
\text { Ansoft Co. }\end{array}$ & FEM & $3 \mathrm{D}$ & Sí & Sí & Sí & Sí & Sí & Automática \\
\hline $\begin{array}{c}\text { Feko [4.53] } \\
\text { EMSS }\end{array}$ & MoM & $3 \mathrm{D}$ & Sí & Sí & Sí & Sí & Sí & $\begin{array}{c}\text { Semi- } \\
\text { automática }\end{array}$ \\
\hline $\begin{array}{l}\text { Fidelity [4.54] } \\
\text { Zeland Softw. }\end{array}$ & FDTD & $3 \mathrm{D}$ & Sí & Sí & Sí & Sí & Sí & $\begin{array}{c}\text { Semi- } \\
\text { automática }\end{array}$ \\
\hline
\end{tabular}

\subsubsection{Simuladores utilizados.}

En este apartado se describen las características de los simuladores em comerciales utilizados para la realización de este trabajo., junto con algunas de sus particularidades y limitaciones.

\section{IE3D.}

IE3D, basado en el MoM, es un programa 2,5D particularmente apropiado para el diseño y simulación de estructuras planas [4.47]. Permite la simulación de estructuras complejas en un tiempo relativamente corto. La discretización de la estructura como combinación de rectángulos y triángulos, se realiza de forma automática. Se puede trabajar con planos de masa tanto infinitos como finitos, aunque en este último caso el tiempo de simulación se alarga de forma considerable. Es posible también definir estructuras metálicas tridimensionales, sin embargo los dieléctricos son siempre capas horizontales infinitas.

Los resultados que se obtienen bastan para caracterizar totalmente el comportamiento de una antena: impedancia, coeficientes de transmisión y de reflexión, distribución de corrientes y de 
tensión, y campos próximos y lejanos. Estos datos se pueden importar y exportar para su procesado con otros programas.

\section{CONCEPT.}

Esta herramienta 3D está basada también en el MoM [4.50]. Permite analizar estructuras complejas y de gran tamaño. La descripción de la estructura ha de suministrarse en forma de archivo en el que se detallas las coordenadas de cada uno de los puntos de simulación. Esto supone un grave problema, ya que CONCEPT carece de un interfaz gráfico fácil de utilizar. En este caso, se utilizó para la introducción de los datos el programa AutoCAD, para el que se disponía de una rutina de conversión apropiada. Del mismo modo, los resultados que se obtienen deben ser postprocesados, para lo que es necesario a menudo utilizar otros programas si se quiere una visualización adecuada de los datos.

También con este simulador se obtienen los principales parámetros de las antenas: impedancia, distribución de corrientes, distribución de campos, diagramas de radiación, etc.

CONCEPT realiza los cálculos en el dominio espectral. Por lo tanto, el tiempo de simulación dependerá del número de frecuencias considerado, ya que para cada punto de frecuencia debe llevar a cabo toda la simulación. Esto no supone sin embargo ningún problema importante, ya que los tiempos de simulación son relativamente cortos, lo que permite utilizar CONCEPT para, por ejemplo, optimizar antenas para terminales móviles.

Un problema que se plantea al utilizar este paquete de simulación es su forma de manejar los materiales dieléctricos. Aparte de un proceso de definición complicado y costoso, basado en la caracterización de cada interfaz entre diferentes medios, el tiempo de cálculo se alarga de forma significativa, y los resultados no son siempre satisfactorios.

Finalmente, hay que tener en cuenta que el número de incógnitas en la matriz de impedancias está limitado a unas 50.000, para evitar tener problemas de desbordamiento de memoria o de convergencia numérica en la resolución del sistema de ecuaciones.

\section{EMPIRE.}

A diferencia de los dos simuladores anteriores, EMPIRE está basado en la FDTD [4.49]. Gracias a su interfaz gráfico, Ganymede, es posible describir estructuras tridimensionales de formas aleatorias, y discretizarlas de modo semi-automático. Implementa un mallado no uniforme que permite analizar estructuras relativamente grandes, en términos de longitud de onda, que contengan detalles que haya que discretizar de forma más fina, sin aumentar considerablemente el tiempo de simulación.

Los resultados que se obtienen bastan para caracterizar tanto el comportamiento de una antena como su interacción con el usuario: impedancia, coeficientes de transmisión y de reflexión, distribución de corrientes y de tensión, campos próximos y lejanos y SAR. 
Por otra parte, la simulación se realiza en el dominio temporal. Los datos en el dominio espectral se obtienen mediante una transformada de Fourier, con lo cual se puede elegir a posteriori el rango de frecuencias que se quiere analizar.

El principal problema se presenta a la hora de estudiar estructuras con superficies redondeadas o con un determinado ángulo respecto a los planos cartesianos. Es necesario entonces recurrir a una discretización muy fina, y aumentar así el tempo de simulación. También se alarga el proceso si se consideran cuerpos de alta constante dieléctrica o estructuras altamente resonantes. En estos casos, la señal temporal decae muy lentamente, y son necesarios muchos más pasos, lo que se traduce en un mayor tiempo de cálculo.

Para el análisis de antenas hay que tener en cuenta un factor adicional: aunque a priori los límites absorbentes se pueden aproximar arbitrariamente a las antenas [4.40], en la práctica para que los resultados de la simulación sean fiables, la estructura debe encontrarse a una determinada distancia mínima de las condiciones de contorno absorbentes. Esto se traduce en un espacio de simulación mucho mayor que la antena en sí, y que requiere por lo tanto mayor capacidad de almacenamiento y tiempo de cálculo.

\subsubsection{Limitaciones de los simuladores.}

Tal y como se ha mencionado, los simuladores em son imprescindibles hoy en día para el diseño de antenas de comunicaciones móviles. Sin embargo, hay que ser conscientes de sus limitaciones, ligadas a la geometría del problema, a las aproximaciones matemáticas realizadas o cuestiones físicas. Estos factores tienen una influencia, directa o indirecta, sobre los requerimientos de memoria y tiempo de cálculo.

El primer límite que se suele alcanzar está relacionado con las propiedades geométricas de la estructura que se quiere analizar: el tamaño de una antena y su complejidad, siempre en relación con la longitud de onda de trabajo. Si es demasiado grande, o contiene elementos que requieren una discretización muy fina, el tempo de cálculo o los requerimientos de sistema (velocidad y capacidad de almacenamiento de datos) pueden hacer inviable su simulación.

También se pueden alcanzar los límites del modelo matemático utilizado. En principio siempre es posible obtener una descripción matemática de una estructura física cualquiera. Sin embargo, la resolución del sistema de ecuaciones resultante alcanza un grado de complejidad tal que resulta imposible resolverlo de forma analítica. Se recurre por lo tanto a métodos numéricos, que conducen a una solución que será siempre, por definición, aproximada. Hay que tener en cuenta, además, que pueden aparecer problemas de inestabilidad numérica al invertir la matriz de impedancias, por ser el sistema de ecuaciones excesivamente grande (porque la estructura es de gran tamaño o complejidad), o debido a la resolución numérica de las ecuaciones de superficie (si se trabaja con superficies de gran tamaño). 
En caso de trabajar con antenas para las que se pueda plantear este problema, es necesario llevar a cabo una simplificación de la estructura y no pretender modelar cada detalle. Por ejemplo, se eliminarán elementos del terminal que tengan poca o nula influencia sobre el funcionamiento de la antena, y se considerarán los elementos dieléctricos (soportes, cubiertas) tan sólo en la fase de refinamiento de los resultados. De lo contrario, se requeriría una discretización de la estructura tan fina que los requerimientos de sistema y de tiempo de cálculo se incrementarían de forma exponencial. Para decidir qué elementos se pueden simplificar o eliminar, el diseñador cuenta sólo con su propia experiencia.

Otra fuente de error en la simulación puede ser un excesivo desequilibrio en el mallado de la estructura. Esto ocurre, por ejemplo, si se utilizan celdas muy pequeñas para caracterizar los detalles de la estructura, pero un mallado muy disperso para las grandes superficies. Se pueden producir entonces reflexiones "fantasma".

Por otra parte, el espacio de simulación nunca es infinito, sino lo que debe ser truncado a cierta distancia de la estructura. La alimentación se considera normalmente una fuente ideal, y a menudo se utilizan fuentes de corriente o de tensión para excitar la estructura. Las líneas de alimentación que existen en la realidad entre la fuente y la antena se suelen ignorar, despreciando así fuentes de error como la desadaptación del generador o las ondas de superficie inducidas en los cables.

Del mismo modo, para las propiedades de los materiales se suelen utilizar valores aproximados. Así, se los considera normalmente materiales sin pérdidas, o isotrópicos. Un error común en las simulaciones, debido a este problema, es un desplazamiento de la frecuencia de resonancia. Este error se podría compensar comparando las simulaciones y las medidas, y modificando los parámetros de entrada de la forma correspondiente [4.55]. Sin embargo esto no es completamente fiable, puesto que en las medidas también existe un margen de incertidumbre.

En la práctica, las antenas utilizadas en terminales de comunicaciones móviles están formadas por piezas metálicas y dieléctricas de forma arbitraria, que el simulador debe ser capaz de modelar. Se debe disponer de un programa capaz de manejar tanto hilos de corriente y superficies metálicas como materiales plásticos.

Para la simulación de estas antenas se requieren además ordenadores con una elevada capacidad de almacenamiento y de procesado, ya que a menudo no se pueden realizar demasiadas simplificaciones en la estructura radiante. El cálculo se puede aligerar, sin embargo, si no se tiene en cuenta la totalidad del terminal. Para obtener la impedancia de entrada, por ejemplo, basta a veces con considerar una parte del entorno. Al contrario, para obtener los diagramas de radiación es imprescindible tener en cuenta la estructura al completo.

En general, los simuladores electromagnéticos permiten alcanzar resultados de gran fiabilidad. Pero, incluso con los programas más sencillos, esto sería imposible sin un poco de conocimiento por parte de usuario acerca de los métodos de cálculo. Así, el software más sofisticado puede llevar 
a errores si no se combina con la experiencia del diseñador.

\subsection{Biblografía.}

[4.1] Bahl, I.J. y Bhartia, P., 'Microstrip Antennas', Artech House, Dedham, MA, 1980.

[4.2] Mosig, J.R. y Gardiol, F.E., 'The Near Field of an Open Microstrip Structure', IEEE Antennas and Propagat. Society. Symp., 1979, pp. 379-382.

[4.3] Agrawal, P.K. y Bailey, M.C., 'An Analysis Technique for Microstrip Antennas', IEEE Trans. on Antennas and Propagat., 1977, vol. 25, n 6, pp. 756-759.

[4.4] Lo, Y.T., Solomon, D. y Richards, W.F., 'Theory and Experiment on Microstrip Antennas', IEEE Trans. on Antennas and Propagat., 1979, vol. 27, n² 2, pp. 137-145.

[4.5] Wheeler, H.A., 'Transmission-Line Properties of Parallel Strips Separated by a Dielectric Sheet', IEEE Trans. on Microwave Theory and Tech., 1965, pp. 172-185.

[4.6] Bahl, I.J., 'Build Microstrip Antennas with Paper-Thin Dimensions', Microwaves, 1979, vol. 18 , pp. 50-63.

[4.7] Pénard, E. y Daniel, J.P., 'Electric and Magnetic Currents in Microstrip Antenna Theory', IEEE Antennas and Propagat. Society. Symp., 1982, pp. 164-167.

[4.8] Hammer,D., Van Bouchaute, D., Verschraeben, D. y Van de Capelle, A., 'Model for Calculating the Radiation Field of Microstrip Antennas', IEEE Trans. on Antennas and Propagat., 1979, vol. 27, n² 2, pp. 267-270.

[4.9] Hammerstad, E.O., 'Computer-Aided Design of Microstrip Couplers with Accurate Discontinuity Models', IEEE Microwave Theory \& Tech. Int. Symp., 1981, pp. 54-56.

[4.10] Kirschning, M., Jansen, R.H. y Koster, N.H.L., 'Accurate Model for Open end Effect of Microstrip Lines', Electronics Lett., 1981, vol. 17, pp. 123-125.

[4.11] Martin, N.M., 'Improved Cavity Model Parameters for Calculation of Resonant Frequency of Rectangular Microstrip Antennas', Electronics Lett., 1988, vol. 24, n 11, pp. 680-681.

[4.12] Getsinger, W., 'Microstrip Dispersion Model', IEEE Trans. on Microwave Theory and Tech., 1973, vol. 21, n 11, pp. 34-39.

[4.13] Khellaf, A., Thouroude, D. y Daniel, J.P., 'Simple Expression of Rectangular Patch's Resistance at Resonance', Electronics Lett., 1990, vol. 26, nº 15, pp. 1188-1190.

[4.14] Benalla, A. y Gupta, K.C., 'Multiport Network Model and Transmission Characteristics of Two-Port Rectangular Microstrip Patch Antennas', IEEE Trans. on Antennas and Propagat., 1988, vol. 36, n 10, pp. 1337-1342. 
[4.15] Gupta, K.C., 'Multiport Network Approach for Modelling and Analysis of Microstrip Patch Antennas and Arrays', en 'Handbook of Microstrip Antennas', editado por J.R. James y P.S. Hall, Peter Peregrinus Ltd, para IEE, vol. 1, Londres, 1989, pp. 455-525.

[4.16] Benalla, A. y Gupta, K.C., 'Multiport Network Model for Rectangular Microstrip Patches Covered with a Dielectric Layer', IEE Proceedings, Part H, 1990, vol. 137, n 6, pp. 377-383.

[4.17] Carver, R.J., 'Practical Analytical Techniques for the Microstrip Antenna', Proc. Workshop on Printed Circuit Antenna Tech., New Mexico State Univ., Las Cruces, Oct. 1979, pp. 7/1-20.

[4.18] Carver, K.R y Mink, J.W., 'Microstrip Antenna Technology', IEEE Trans. on Antennas and Propagat., 1981, vol. 29, $\mathrm{n}^{\circ}$ 1, pp. 2-23.

[4.19] Gupta, K.C., 'Two-Port Transmission Characteristics of Rectangular Microstrip Patch Radiators', IEEE Antennas and Propagat. Society. Symp.,1985, pp. 71-74.

[4.20] Benalla, A. y Gupta, K.C., 'Transmission Line Model for 2-Port Rectangular Microstrip Patches with Ports at the Non-Radiating Edges', Electronics Lett., 1987, vol. 23, pp. 882-884.

[4.21] Derneryd, A., 'Linearly Polarised Microstrip Antennas', IEEE Trans. on Antennas and Propagat., 1976, vol 24, pp. 846-851.

[4.22] Lier, E., 'Improved Formulas for Input Impedance of Coax-Fed Microstrip Patch Antennas', IEE Proc. H, $1982 \mathrm{~m} 129$, pp. 161-164.

[4.23] James, J.R., Hall, P.S., Wood, C. y Henderson, A., 'Some Recent Developments in Microstrip Antenna Design', IEEE Trans. on Antennas and Propagat., 1981, vol. 29, nº 1, pp. 124-128.

[4.24] Chang, D.C., 'Analytical Theory of an Unloaded Rectangular Microstrip Patch', IEEE Trans. on Antennas and Propagat., 1981, vol. 29, n 1, pp. 54-62.

[4.25] Pues, H. y Van de Capelle, A., 'Accurate Transmission-Line Model for the Rectangular Microstrip Antenna', IEE Proc., 1984, vol. 131, Part H, pp. 334-340.

[4.26] Mosig, J. R., 'Arbitrarily Shaped Microstrip Structures and their Analysis with a Mixed Potential Integral Equation', IEEE Transactions on Microwave Theory and Techniques, 1988, vol.36, nº 2, pp. 314-323.

[4.27] Mosig, J.R., Hall, R.C. y Gardiol, F.E., 'Numerical Analysis of Microstrip Patch Antennas', en 'Handbook of Microstrip Antennas', editado por James, J.R. y Hall, P.S., Peter Peregrinus Ltd, para IEE, Londres, 1989.

[4.28] Poggio, A.J. and Miller, E.K., en 'Computer Techniques for Electromagnetics', editado por Mittra, R., Pergamon Press, 1973.

[4.29] Mohamadian, A.H., 'Integral Equation Formulation for Printed Antennas', IEEE Antennas and Propagat. Society. Symp., 1987, pp. 606-609.

[4.30] Barlately, L., Mosig, J.R. y Sphicopoulos, T. , 'Analysis of Stacked Microstrip Patches with a Mixed Potential Integral Equation', IEEE Trans. on Antennas and Propagat., 1990, vol. 38, 
$\mathrm{n}^{\circ} 5$, pp. 608-615.

[4.31] Sorrentino, R., Alessandri, F., Mongiardo, M., Avitabile, G. y Roselli, L., 'Full-Wave Modelling of Via Hole Grounds in Microstrip by Three-Dimensional Mode Matching Technique', IEEE Trans. on Microwave Theory and Tech., 1992, vol. 40, n 12, pp. 22282234.

[4.32] Becks, T. y Wolff, I.,'Analysis of 3-D Metallization Structures by a Full-Wave Spectral Domain Technique', IEEE Trans. on Microwave Theory and Tech., 1992, vol. 40, n 12, pp. 2219-2227.

[4.33] Rautio, J.C. y Harrington, R.F., 'An Efficient Electromagnetic Analysis of Arbitrary Microstrip Circuits', IEEE Microwave Theory and Techniques International Symposium Digest, 1987, pp. 295-298.

[4.34] Harrington R.F., 'Field Computation by Moment Method', Malabar, Robert E. Krieger Publishing, 1982

[4.35] Wang, J.J.H., 'Generalized Moment Methods in Electromagnetics', John Wiley \& Sons, 1991.

[4.36] Nakano, H., Kerner, S.R. y Alexopoulos, N.G., 'The Moment Method Solution for Printed Antennas of Arbitrary Configuration', IEEE Antennas and Propagat. Society. Symp., 1987, pp. 1016-1019.

[4.37] Yee K.S., 'Numerical Solution of Initial Boundary Value Problems Involving Maxwell's Equations in Isotropic Media', IEEE Trans. on Antennas and Propagat., 1966, vol. 14, n 3 , pp. 302-307.

[4.38] Kunz K.S. y R. J. Luebbers, 'The Finite Difference Time Domain Method for Electromagnetics', CRC Press, Boca Raton, 1993.

[4.39] Mur G., 'Absorbing Boundary Conditions for Finite-Difference Approximation of the TimeDomain Electromagnetic-Field Equations', IEEE Trans. on Electromagnetic Compatibility, 1981, n 23, pp. 1073-1077

[4.40] Berenger J.P., 'A Perfectly Matched Layer for the Absorption of Electromagnetic Waves', Journal of Computational Physics, 1994, $\mathrm{n}^{\circ}$ 114, pp. 185-200

[4.41] Hoefer W.J.R., 'The Transmission-Line-Matrix Method - Theory and Applications', IEEE Trans. on Microwave Theory and Tech., 1985, vol. 35, $\mathrm{n}^{\circ}$ 4, pp. 882-893

[4.42] Johns P.B., 'A Symmetrical Condensed Node for the TLM-Method', IEEE Trans. on Microwave Theory and Tech., 1987, vol. 35, n 4, pp. 379-377

[4.43] Baker B.B. y Copson, E.T., 'The Mathematical Theory of Huygens' Principle', London, 1969.

[4.44] Huber, C., Krumpholz, M. y Russer, P., 'Dispersion in Anisotropic Media Modeled by 
Three-Dimensional TLM', IEEE Trans. on Microwave Theory and Tech., 1995, vol. 43, ${ }^{\circ}$ 6, pp. 1923-1934

[4.45] Jansen, M. A. y Rahmat-Samii, Y., 'Interaction of Handsets Antennas and a Human in P.C.', Proceedings of the IEEE, 1995, vol. 83, $\mathrm{n}^{\circ} 1$, pp. 7-17.

[4.46] Sánchez-Hernández, D., Robertson, I.D. y Sinclair, C., 'Electromagnetic Simulation', IEE Colloquium on MMICs, 1994, pp. 5/1-5/7.

[4.47] Zheng, J.X., 'Electromagnetic Simulation of Microstrip Patch Antennas', IEEE Antennas and Propagat. Society. Symp., 1994, pp. 318-321]

[4.48] Allen, R., Mallik, A. y Johns, P. 'Numerical Results for the Symmetrical Condensed TLM Node', IEEE Trans. on Microwave Theory and Tech., 1987, vol. 35, n 4, pp. 378-382.]

[4.49] 'User and Reference Manual for the 3D-EM Time-Domain Simulator EMPIRE', IMST $\mathrm{GmbH}, 2002$, www.empire.de.

[4.50] 'CONCEPT II', Universidad de Hamburgo-Harburg, 1997.

[4.51] http://www.ansoft.com.

[4.52] http://www.remcominc.com.

[4.53] http://www.emss.de.

[4.54] http://www.zeland.com.

[4.55] Martínez-Vázquez, M., Catalá, J.M. y Sánchez-Hernández, D., 'Quantification of Errors in the Prediction of Resonant Frequencies for Printed Antennas using Commercial Electromagnetic Simulators through the Utilisation of Measured Substrate Dielectric Properties', Millennium Conf. on Antennas and Propagation AP2000, 2000. 


\section{Capítulo 5: ANTENAS IMPRESAS DE DOBLE BANDA.}

La formulación de un problema, es más importante que su solución.

Albert Einstein

\subsection{INTRODUCCIÓN.}

Con la llegada de los sistemas móviles de tercera generación se hace necesario el desarrollo de terminales multibanda-multifunción. Como las frecuencias de los sistemas considerados pueden estar bastante alejadas unas de otras y es necesario que las antenas utilizadas operen en todas las bandas de forma eficiente, se suele recurrir a estructuras radiantes duales.

Surge entonces la necesidad de emplear nuevas técnicas que permitan conseguir la operación en banda dual sin incrementar el volumen total de la antena [5.1]-[5.2]. Una antena convencional se puede transformar en dual con la inclusión de un elemento (filtro, cortocircuito,...) que introduzca una segunda resonancia a la frecuencia deseada, sin necesidad de incrementar el tamaño total de la estructura o de duplicar el sistema. La extensión del sistema a una nueva banda de operación se puede hacer entonces de forma sencilla y económica.

Con el fin de desarrollar elementos radiantes para terminales móviles multibanda-multifunción, se estudia aquí la posibilidad de obtener antenas que operen en dos bandas de frecuencia mediante la inserción de filtros espolón en parches radiantes.

\subsection{EL FILTRO ESPOLÓN.}

\subsubsection{Estructuras multibanda.}

Como se ha mencionado anteriormente, los terminales móviles actuales operan, como mínimo, en dos bandas de frecuencias distintas. Para ello se prefiere utilizar una única antena, con un solo punto de alimentación. Para lograrlo, se puede recurrir a diferentes métodos: utilización de estructuras multicapa [5.3], introducción de parches parásitos acoplados al parche radiante 
principal [5.4], [5.5], utilización de geometrías logoperiódicas o quasi-logoperiódicas [5.6], [5.7], parches fractales [5.8], [5.9], etc. Sin embargo, la mayoría de estas técnicas conllevan un incremento del tamaño total de la antena.

Para evitar este aumento de tamaño, es posible lograr un modo de operación multifrecuencia cargando el parche. Para ello se recurre a elementos reactivos, como pines metálicos [5.10], diodos [5.11], [5.12], huecos de aire (air gap) en el dieléctrico [5.13], [5.14] y stubs [5.15]. Con ello, sin embargo, se complica y encarece el proceso de fabricación.

\subsubsection{Filtro de banda eliminada espolón.}

Una alternativa para lograr una doble resonancia con único parche sería insertar en su perímetro un filtro espolón. De hecho, se ha demostrado que esto es posible [5.16] para antenas rectangulares, si el filtro está ubicado en el borde radiante opuesto al punto de alimentación, tal y como se representa en la Figura 5.1. Con ello se logran dos resonancias separadas, sin por ello aumentar el tamaño total de la antena. Así, el tamaño del parche se determina para obtener una resonancia a la frecuencia de operación más elevada, mientras que las dimensiones del filtro definen la más baja.

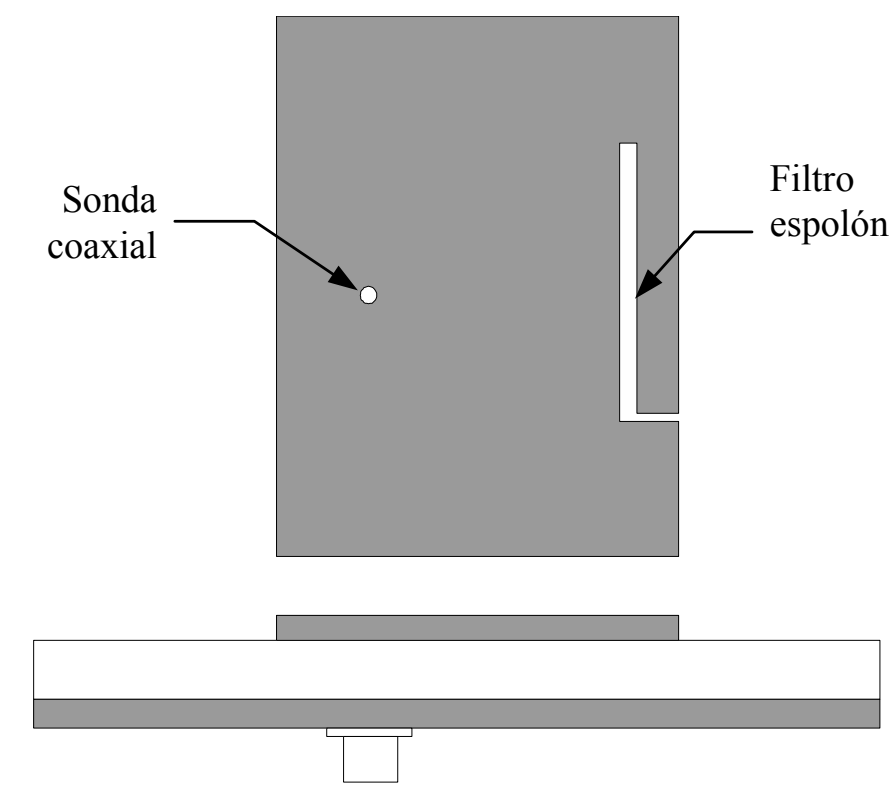

Figura 5.1: Antena impresa dual rectangular con filtro espolón embedido.

El filtro de banda eliminada espolón fue propuesto por Bates [5.17] para aplicaciones que requerían un ancho de banda de alrededor del 10\%. El filtro se puede integrar en estructuras de pequeño tamaño, como por ejemplo una línea microtira, lográndose así estructuras extremadamente compactas. La respuesta del filtro es prácticamente no dispersiva, puesto que la respuesta se reproduce en cada múltiplo impar de la frecuencia de diseño.

La estructura del filtro se muestra en la Figura 5.2. Está formado por dos líneas acopladas de 
longitud aproximadamente igual a un cuarto de la longitud de onda a la frecuencia de resonancia. Una de las dos líneas está en circuito abierto en uno de sus extremos, mientras que ambas están conectadas entre sí en el extremo opuesto. El filtro se puede considerar igualmente como un stub paralelo a una línea de transmisión, con el hueco entre ambos lo suficientemente pequeño como para que exista un acoplamiento entre ambos. Una de las ventajas de utilizar esta estructura es que se consigue evitar la aparición de ondas de superficie. La frecuencia de operación está determinada por la longitud a del stub y por el tamaño del hueco, $b$.

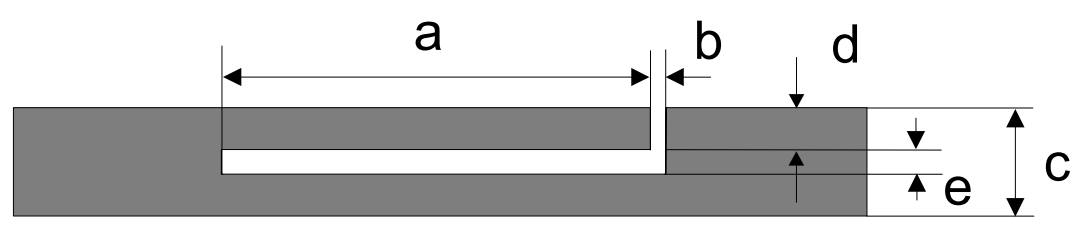

Figura 5.2: Estructura del filtro de banda eliminada espolón.

Este tipo de filtros ha sido utilizado también con éxito para el diseño de antenas impresas con polarización circular. En [5.18] se presenta una antena impresa equitriangular, con un filtro espolón embedido en cada uno de los lados. Si uno de los filtros tiene una longitud ligeramente diferente de los otros dos, se pueden excitar dos nuevos modos con polarización ortogonal, lográndose así una antena de polarización circular.

Con respecto a otros filtros para estructuras impresas, el filtro espolón presenta una serie de ventajas:

$\infty$ La estructura final es compacta.

- Su comportamiento es prácticamente no dispersivo, la respuesta se repite de forma casi exacta para los múltiplos impares de la frecuencia de diseño $\mathrm{f}_{0}$.

$\infty$ Las pérdidas de retorno en la banda eliminada son de alrededor de $1 \mathrm{~dB}$, mientras que con filtros de líneas acopladas convencionales se elevaban a unos 2,5 dB. Así, un filtro espolón refleja un $78 \%$ de la potencia incidente, mientras que los filtros de líneas acopladas no reflejan más que el $56 \%$.

\subsubsection{Análisis del filtro.}

El comportamiento de un par de líneas acopladas como las utilizadas para formar el filtro se puede partiendo de la matriz de impedancias propuesta por Jones y Bolljahn [5.19] para líneas impresas con propagación TEM. Sin embargo, en líneas microtira la propagación no es TEM, sino cuasi-TEM. La estructura es dispersiva y las velocidades de fase son distintas para los modos par e impar. A pesar de todo, para frecuencias inferiores a $2 \mathrm{GHz}$ se puede asumir que la propagación es TEM, con lo cual se pueden utilizar las expresiones propuestas por Bryant y Weiss [5.20] o Judd [5.21] para determinar las impedancias y las velocidades de fase. Por encima de los $2 \mathrm{GHz}$, la dispersión empieza a ser significativa. Para estos casos hay que recurrir al método de análisis desarrollado por Getsinger 
[5.22].

Un análisis detallado del comportamiento del filtro se puede hallar en [5.17]. En él se tiene en cuenta la capacidad que aparece entre la línea y el final del espolón. Es el modo impar el que determina la energía almacenada por la estructura, ya que los campos de este modo están confinados en la proximidad de los conductores, y concentrados en el hueco entre las dos tiras metálicas. Por contra, los campos relacionados con el modo par tienden a alejarse de los conductores, y por lo tanto se ven más afectados por la presencia de objetos en la proximidad de la estructura [5.23]. Por lo tanto, el filtro espolón es relativamente poco sensible a la presencia de elementos externos. El efecto de estos sólo se aprecia si están situado a muy poca distancia del filtro.

El cuadripolo equivalente del filtro espolón se presenta en la Figura 5.3. En ella se puede apreciar cómo el puerto 3 del cuadripolo equivalente a dos líneas acopladas está terminado por una capacidad, $\mathrm{C}_{\text {end }}$, que representa la capacitancia que aparece en el extremo del espolón.

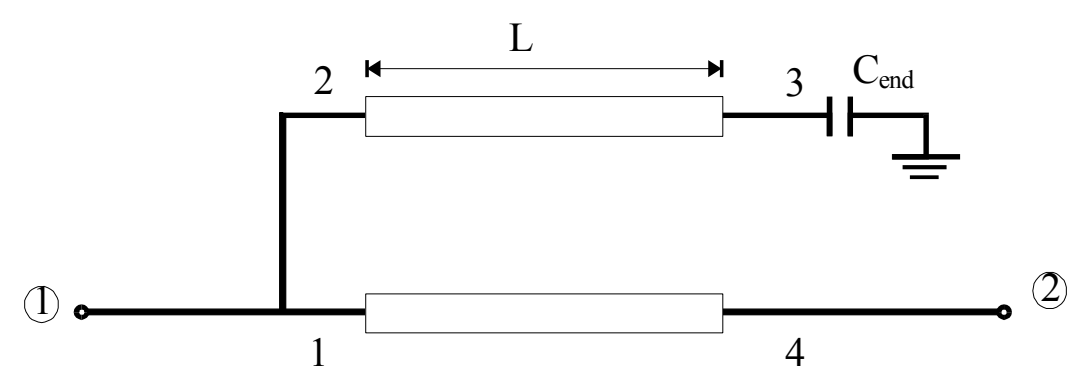

Figura 5.3: Modelo de cuadripolo.

Sin embargo, para simplificar el análisis, se ha desarrollado igualmente el modelo de líneas presentado en la Figura 5.4.

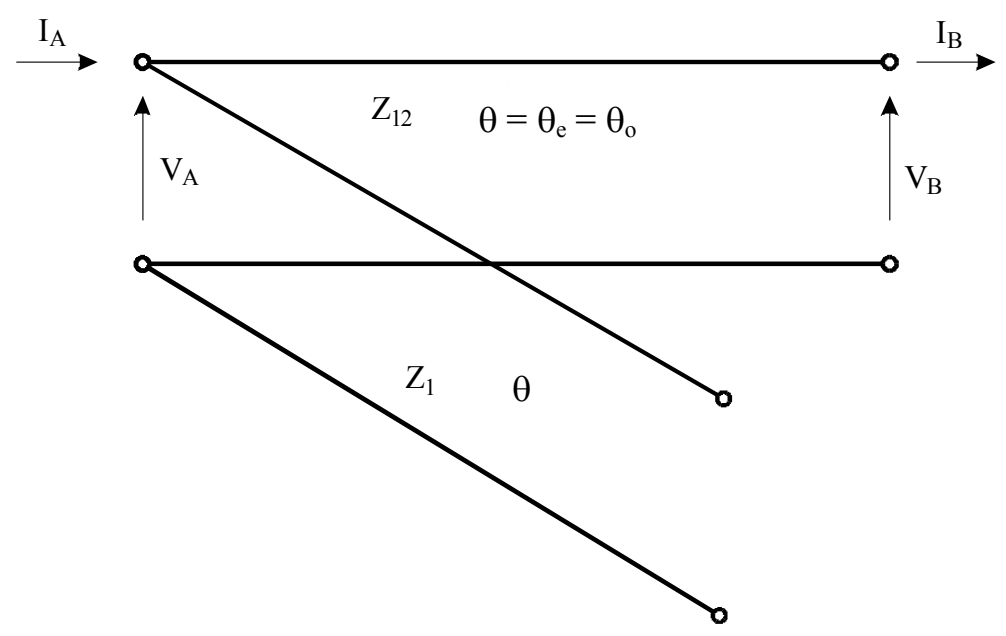

Figura 5.4: Circuito equivalente de una sección.

Considerando que la estructura es no dispersiva, con velocidades de fase iguales para los modos par e impar, se puede asimilar el filtro espolón a un tramo de línea de impedancia característica $Z_{12}$ en paralelo con un stub en circuito abierto de impedancia característica $Z_{1}$ [5.20]. Las impedancias 
características de estas líneas se calculan como:

$$
\begin{gathered}
Z_{12}=\frac{Z_{\mathrm{oo}}+Z_{\mathrm{oe}}}{2} \\
Z_{1}=\frac{Z_{\mathrm{oe}}}{Z_{\mathrm{oo}}}\left(\frac{Z_{\mathrm{oo}}+Z_{\mathrm{oe}}}{2}\right)
\end{gathered}
$$

Siendo $\mathrm{Z}_{\mathrm{oo}} \mathrm{y}_{\mathrm{oe}}$ las impedancias de los modos par e impar de las líneas acopladas.

La capacidad que aparece en el extremo del stub, representada por $\mathrm{C}_{\text {end }}$, tiene como efecto aumentar la longitud efectiva del filtro. Su valor se puede obtener modificando el modelo de capacidad desarrollado por Garg [5.24], y sustituyéndolo por los modelos propuestos por Benedek [5.25], tal y como se muestra en la Figura 5.5. Esta transformación es posible porque, a la frecuencia de resonancia, no fluye corriente a través del hueco.
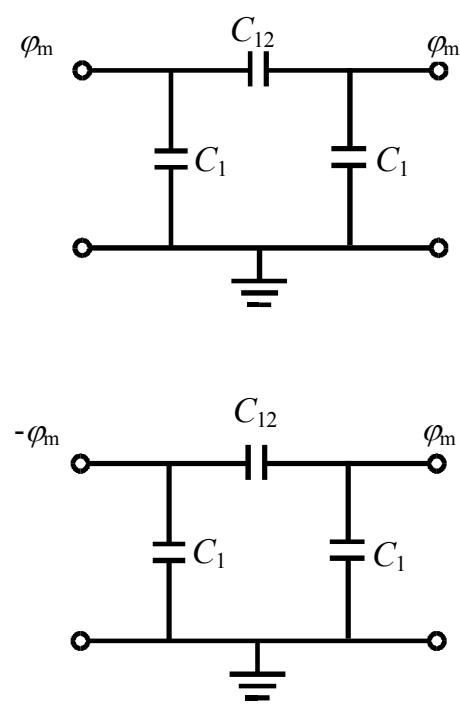

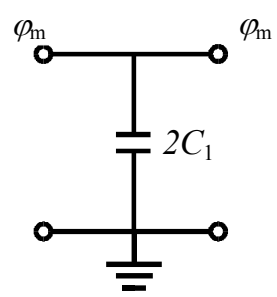

(a)

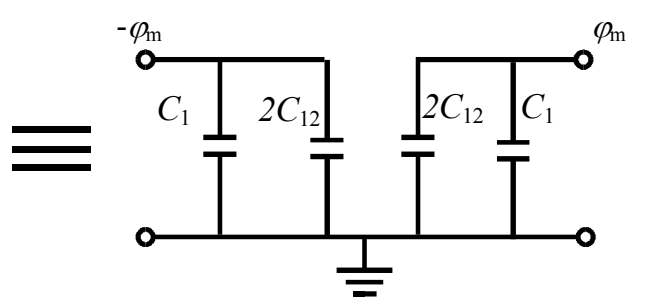

(b)

Figura 5.5: Circuito equivalente de la capacidad del hueco (gap capacity) de las líneas microtira con:

(a) excitación simétrica $\left(\mathrm{C}_{\text {even }}\right)$

(b) excitación asimétrica $\left(\mathrm{C}_{\text {odd }}\right)$

Los valores de $\mathrm{C}_{1}$ y $\mathrm{C}_{12}$ se calculan como [5.24]:

$$
\begin{gathered}
\mathrm{C}_{1}=\frac{\mathrm{C}_{\text {even }}}{2} \\
\mathrm{C}_{12}=\frac{\mathrm{C}_{\text {odd }}-\mathrm{C}_{1}}{2}
\end{gathered}
$$

Se obtiene por lo tanto:

$$
\begin{gathered}
\mathrm{C}_{\text {even }}=2 \mathrm{C}_{1} \\
\mathrm{C}_{\text {odd }}=\mathrm{C}_{1}+2 \mathrm{C}_{12} \\
\mathrm{C}_{\text {end }}=\mathrm{C}_{\text {odd }}
\end{gathered}
$$

Así, hay que tener en cuenta $\mathrm{C}_{\text {end }}$ al calcular la longitud total del espolón, a, definida como: 


$$
\mathrm{a}=\frac{\mathrm{c}}{4 \mathrm{f}_{0} \sqrt{\mathrm{K}_{\text {effo }}}}-\Delta \mathrm{l}
$$

Donde $\mathrm{K}_{\text {effo }} \mathrm{y} \Delta \mathrm{l}$ representan la constante dieléctrica efectiva del modo impar y la extensión de longitud efectiva debida al hueco b, respectivamente [5.20]. Sus expresiones exactas se describen más adelante, junto con el procedimiento de diseño del filtro.

Existen también estudios para los casos de estructuras dispersivas, que establecen otro modelo circuitales [5.26], presentado en la Figura 5.6. En este modelo se aproxima el filtro por una línea de transmisión de impedancia característica $Z_{1}$ y longitud eléctrica $\theta_{\mathrm{e}}$, en serie con un stub en cortocircuito, de impedancia característica $Z_{2}$ y longitud eléctrica $\theta_{0}$. $Z_{1}$ y $Z_{2}$ se definen como:

$$
\begin{aligned}
& Z_{1}=\frac{Z_{\mathrm{oe}}}{2} \\
& Z_{2}=\frac{Z_{\mathrm{oo}}}{2}
\end{aligned}
$$

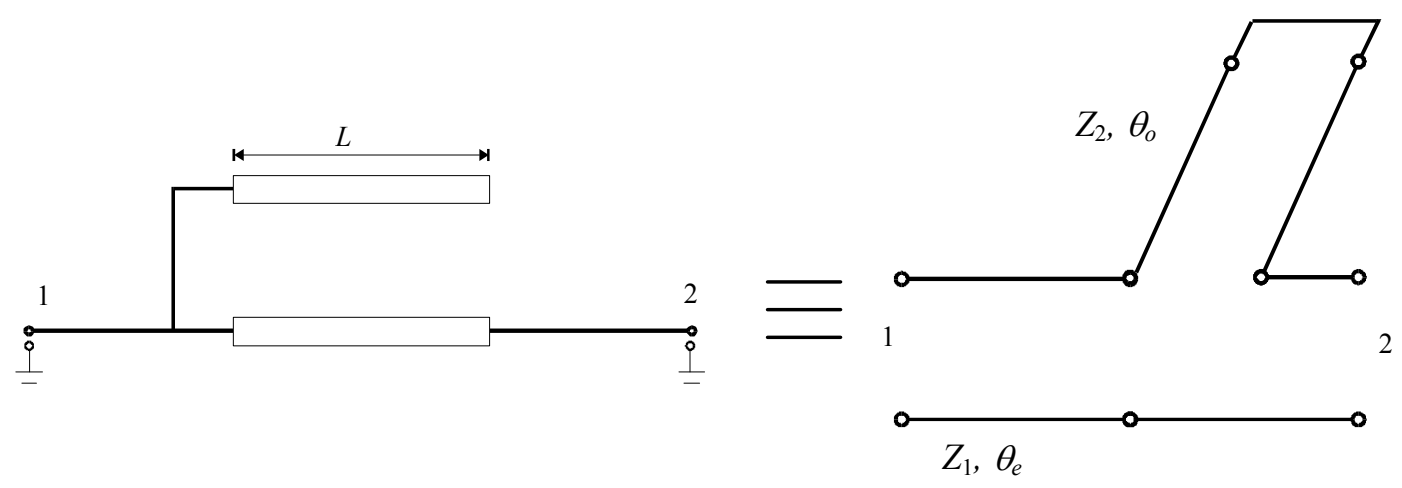

Figura 5.6: Circuito equivalente exacto de un filtro espolón.

Este modelo es, sin embargo, más difícil de aplicar, y por lo general no suele ser necesario recurrir a él, puesto que el modelo no dispersivo permite obtener resultados bastante exactos cuando el hueco es de menos de $100 \mu \mathrm{m}$, tal y como se demuestra en [5.17].

\subsection{ANTENA IMPRESA RECTANGULAR DUAL.}

\subsubsection{Parche rectangular.}

La forma más simple de una antena impresa es el parche rectangular presentado en la Figura 5.7. En el Capítulo 3 se presentaba un análisis detallado de esta estructura. En la práctica, para el primer modo resonante $\mathrm{TM}_{10}$, este parche se diseña fácilmente siguiendo los pasos descritos en [5.27]. 


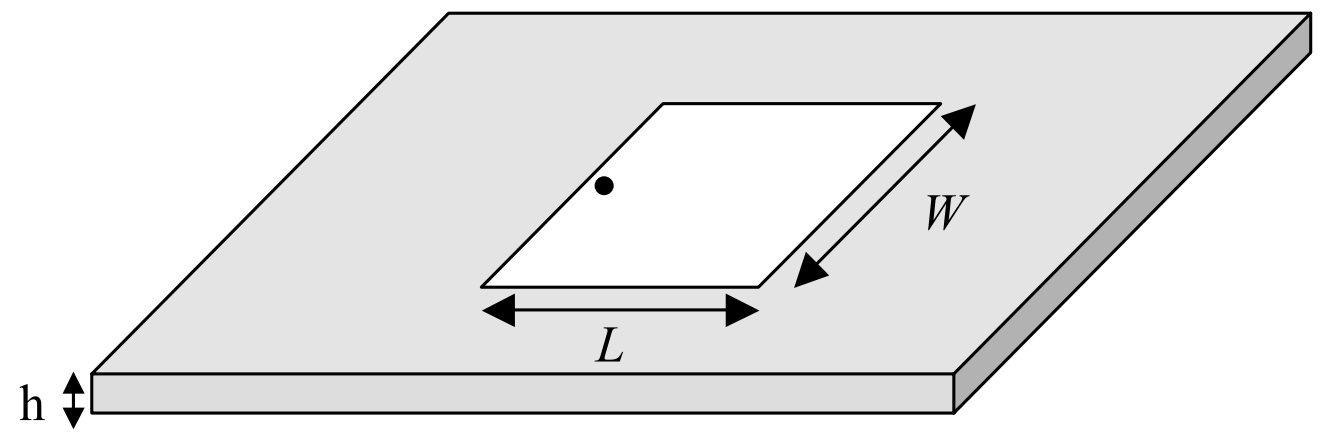

Figura 5.7: Parche impreso rectangular.

En primer lugar, se determina el ancho del parche para la frecuencia de operación a partir de la permitividad del dieléctrico como:

$$
\mathrm{W}=\frac{\mathrm{c}}{2 \mathrm{f}_{\mathrm{r}}}\left(\frac{\varepsilon_{\mathrm{r}}+1}{2}\right)^{-1 / 2}
$$

El parámetro $\mathrm{W}$ no influye en la frecuencia de resonancia, por lo que en principio se podría variar. Sin embargo, si se toma $\mathrm{W}$ menor, disminuye le eficiencia.

Por contra, con W mayor se obtiene mayor eficiencia, pero pueden aparecer modos de orden superior que modificarían las distribuciones de campos.

La longitud del parche para una determinada frecuencia de operación se calcula como:

$$
\mathrm{L}=\frac{\mathrm{c}}{2 \mathrm{f}_{\mathrm{r}} \sqrt{\varepsilon_{\mathrm{e}}}}-2 \Delta 1
$$

con:

$$
\begin{gathered}
\Delta \mathrm{l}=\frac{0,412 \mathrm{~h}\left(\varepsilon_{\mathrm{e}}+0,3\right)\left(\frac{\mathrm{W}}{\mathrm{h}}+0,264\right)}{\left(\varepsilon_{\mathrm{e}}-0,258\right)\left(\frac{\mathrm{W}}{\mathrm{h}}+0,8\right)} \\
\varepsilon_{\mathrm{e}}=\frac{\varepsilon_{\mathrm{r}}+1}{2}+\frac{\varepsilon_{\mathrm{r}}-1}{2}\left(1+\frac{12 \mathrm{~h}}{\mathrm{~W}}\right)^{-1 / 2}
\end{gathered}
$$

Para la implementación de la antena se utiliza un substrato dieléctrico bastante común: GML 1000 de GIL Technologies, cuyas características se detallan en la Tabla 5.1 [5.28].

Tabla 5.1: Características del substrato para $\mathrm{f}=1 \mathrm{GHz}$.

\begin{tabular}{|c|c|}
\hline \multicolumn{2}{|c|}{ GML 1000 } \\
\hline $\mathrm{h}$ & $1,524 \mathrm{~mm}$ \\
\hline$\varepsilon_{\mathrm{r}}$ & 3,05 \\
\hline $\operatorname{tg} \delta$ & 0,003 \\
\hline
\end{tabular}


Las dimensiones que se obtienen aplicando este procedimiento para el diseño de un parche rectangular resonante a $1,8 \mathrm{GHz}$ se muestran en la Tabla 5.2.

Tabla 5.2: Dimensiones del parche rectangular.

\begin{tabular}{|c|r|}
\hline \multicolumn{2}{|c|}{ Parche rectangular } \\
\hline $\mathrm{W}(\mathrm{mm})$ & 58,6 \\
\hline $\mathrm{L}(\mathrm{mm})$ & 47,1 \\
\hline
\end{tabular}

\subsubsection{Diseño del filtro.}

Se pretende originar una segunda resonancia utilizando un filtro espolón paralelo al lado de la antena. El filtro espolón se diseña para la frecuencia de operación definida por el estándar GSM. El material que se utiliza es el mismo que en el apartado anterior. Los requisitos que debe cumplir el filtro se detallan en la Tabla 5.3.

Tabla 5.3: Especificaciones de diseño del filtro espolón.

\begin{tabular}{|c|c|}
\hline Tipo de filtro & Chebycheff \\
\hline Frecuencia central $\left(\mathrm{f}_{0}\right)$ & $0.925 \mathrm{GHz}$ \\
\hline Ancho de banda $\left(\mathrm{f}_{2}-\mathrm{f}_{1}\right)$ & $1 \mathrm{GHz}$ \\
\hline Atenuación a $\mathrm{f}_{0}-0,25 \mathrm{GHz}$ & $-30 \mathrm{~dB}$ \\
\hline Rizado en la banda de paso & $0.01 \mathrm{~dB}$ \\
\hline
\end{tabular}

Una explicación detallada del procedimiento de diseño se puede hallar en [5.26]. Siguiendo este procedimiento, y con las especificaciones de la Tabla 5.3, las dimensiones para un filtro espolón de frecuencia central $925 \mathrm{MHz}$ se muestran en la Tabla 5.4.

Tabla 5.4: Dimensiones del filtro espolón.

\begin{tabular}{|c|c|}
\hline \multicolumn{2}{|c|}{ Parámetro } \\
\hline $\mathrm{a}$ & $50,63 \mathrm{~mm}$ \\
\hline $\mathrm{b}$ & $0,1 \mathrm{~mm}$ \\
\hline $\mathrm{d}$ & $1,68 \mathrm{~mm}$ \\
\hline $\mathrm{e}$ & $0,15 \mathrm{~mm}$ \\
\hline
\end{tabular}

\subsubsection{Parche dual.}

Para lograr una segunda frecuencia de resonancia en el parche rectangular, sin por ello modificar sus dimensiones totales, se inserta en el mismo el parche espolón diseñado en el apartado 5.3.2. Al 
ser el filtro paralelo a los bordes del parche, su influencia sobre el modo fundamental es insignificante, mientras que el nuevo modo generado tendrá características similares a las del modo principal [5.29]-[5.30].

La estructura de la antena se puede apreciar en la Figura 5.8, mientras que en la Figura 5.9 se muestra su implementación sobre el substrato GML 1000 de GIL Technologies.

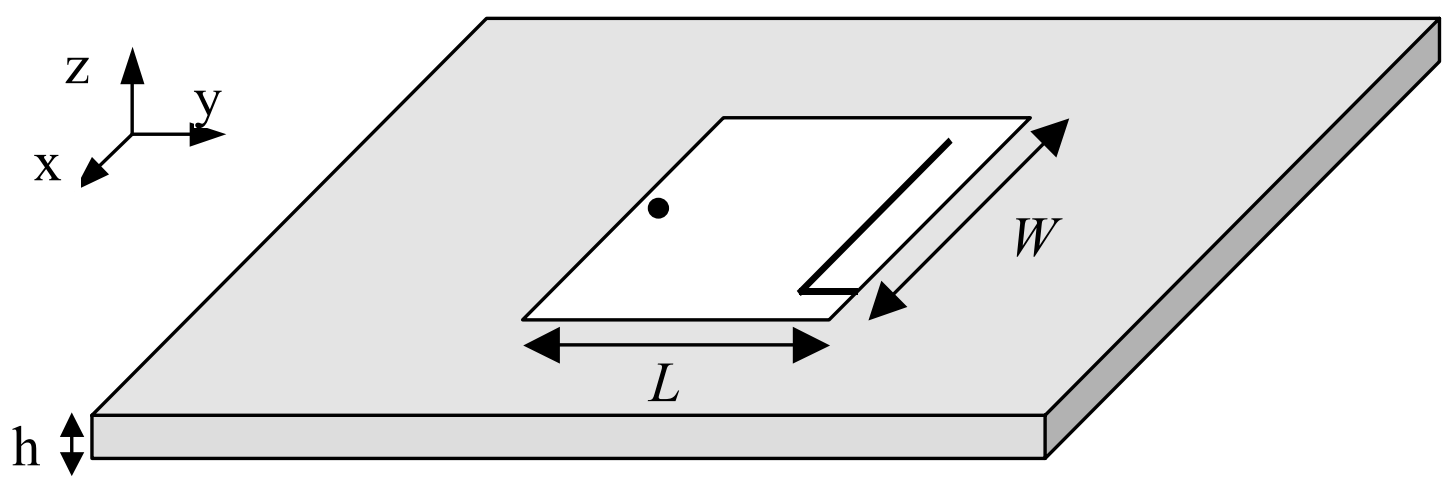

Figura 5.8: Estructura del parche dual con filtro espolón.

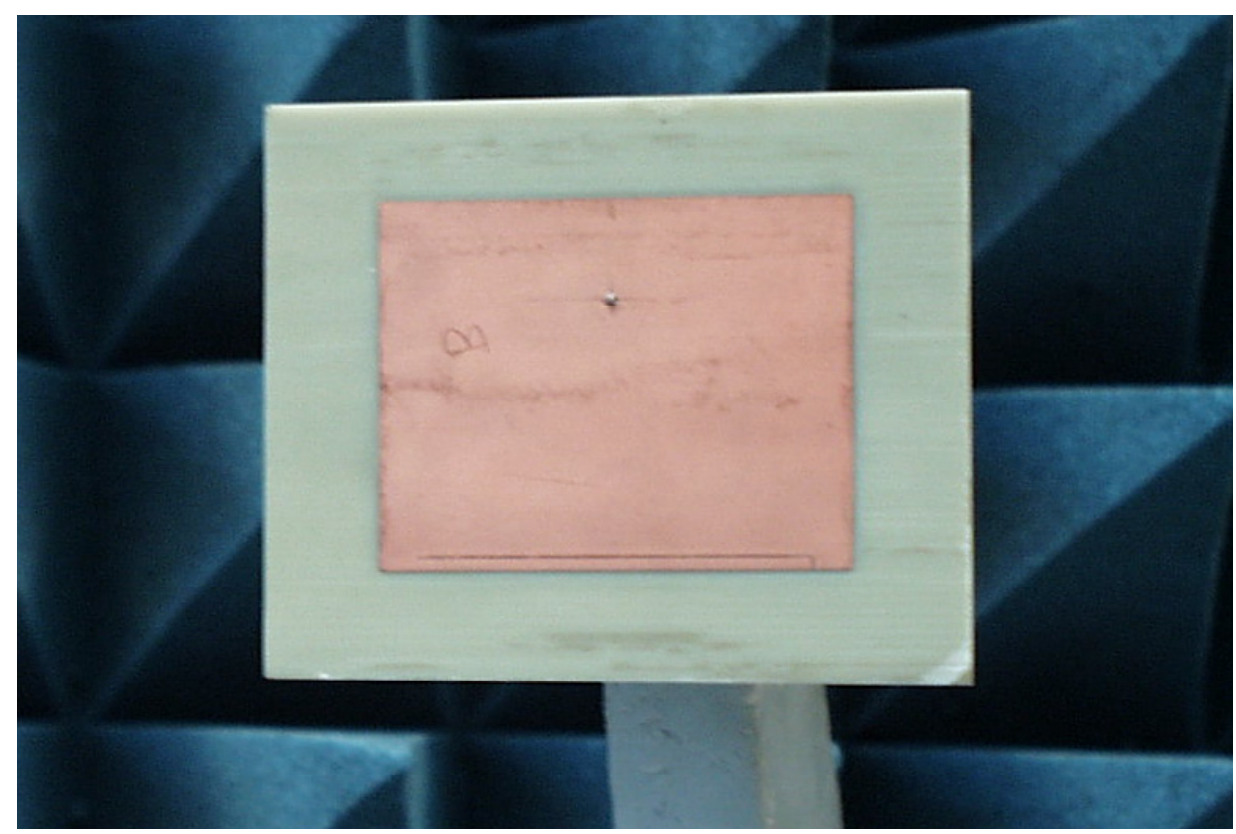

Figura 5.9: Implementación del parche con filtro espolón.

Los resultados obtenidos, tanto en la simulación como en las medidas, se muestran en la Figura 5.10. Se puede apreciar cómo, aunque las frecuencias de resonancia se ajustan a las deseadas, las pérdidas de retorno y los anchos de banda no son los necesarios para cumplir con los estándares de comunicaciones móviles. Por otra parte, la antena es demasiado grande para poder ser integrada en un terminal bde comunicaciones móviles como los existentes en la actualidad, con lo que habrá que recurrir a soluciones alternativas, tal y como se verá en los siguientes apartados. 


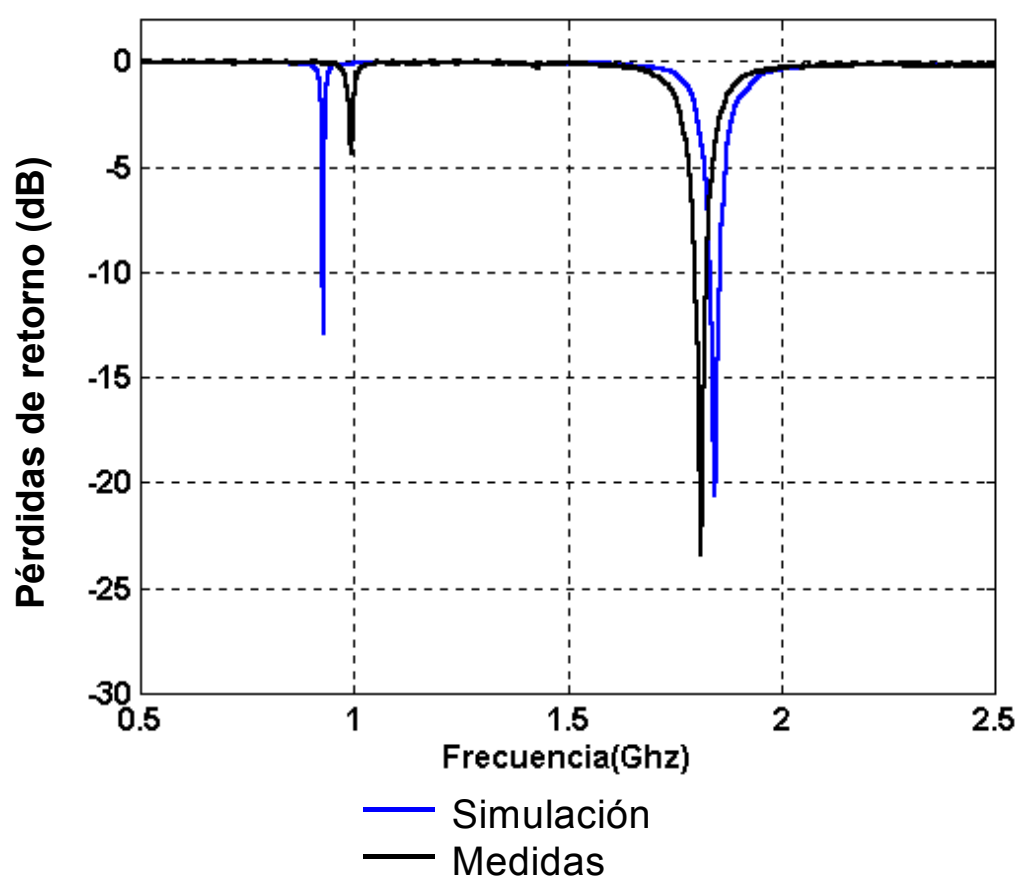

Figura 5.10: Pérdidas de retorno del parche dual.

En la Figura 5.11 se observa cómo tanto la distribución de campo eléctrico como el diagrama de radiación del parche en la banda de DCS no se han visto alterados significativamente por la introducción del filtro espolón. Para la banda de GSM, sin embargo, se aprecia que la nueva resonancia corresponde al filtro, mientras que el diagrama de radiación es más irregular.
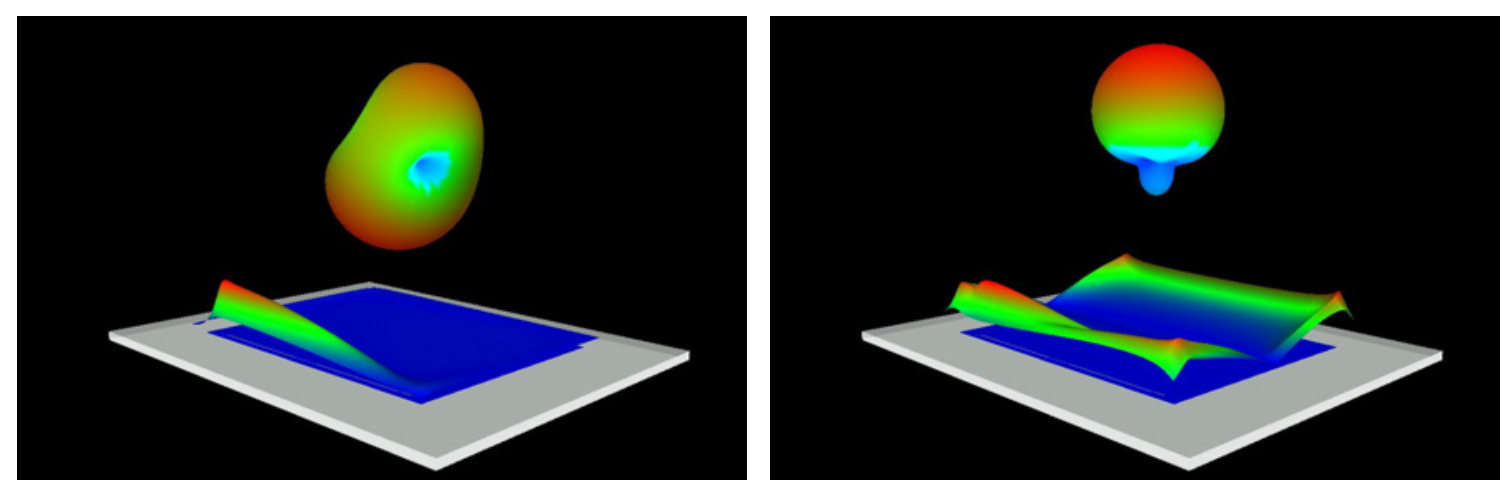

Figura 5.11: Distribuciones de campo eléctrico y diagramas de radiación del parche rectangular dual con filtro espolón en las bandas de GSM (izquierda) y DCS (derecha).

Estas características de la antena se observan también en los diagramas de radiación medidos, recogidos en la Tabla 5.5. Las medidas se llevaron a cabo teniendo en cuenta el sistema de coordenadas de la Figura 5.8.

Resultados similares se pueden obtener aplicando esta técnica a parches de diferentes geometrías [5.31], como parches equitriangulares, circulares o con forma de pajarita (bow-tie). 


\subsection{La PIFA (PLANAR INVERTED-F ANTENNA).}

En el apartado anterior se presentaba una posible forma de fabricación de antenas de doble banda para comunicaciones móviles. Sin embargo no se lograba alcanzar el ancho de banda necesario para estas aplicaciones. Una solución viable es aumentar la altura del parche radiante respecto del plano de masa, utilizando substratos más espesos. El inconveniente entonces sería la posible aparición de ondas de superficie que deteriorarían el funcionamiento de la antena.

La solución más extendida consiste en eliminar el substrato dieléctrico, y trabajar únicamente con chapa metálica. Así, se tiene una gran flexibilidad en el diseño, ya que se pueden desarrollar estructuras planas o tridimensionales. Con ello se pueden idear estructuras radiantes que se adapten al escaso volumen disponible dentro del terminal, y que constituye una de las limitaciones fundamentales para el diseño de las antenas integradas en terminales de comunicaciones móviles. Accesoriamente se puede utilizar un soporte de plástico para asegurar la estabilidad de la estructura, aunque también existen casos en los que la antena se fija directamente a la cubierta posterior del móvil.

Tabla 5.5: Diagramas de radiación del parche rectangular con filtro espolón.

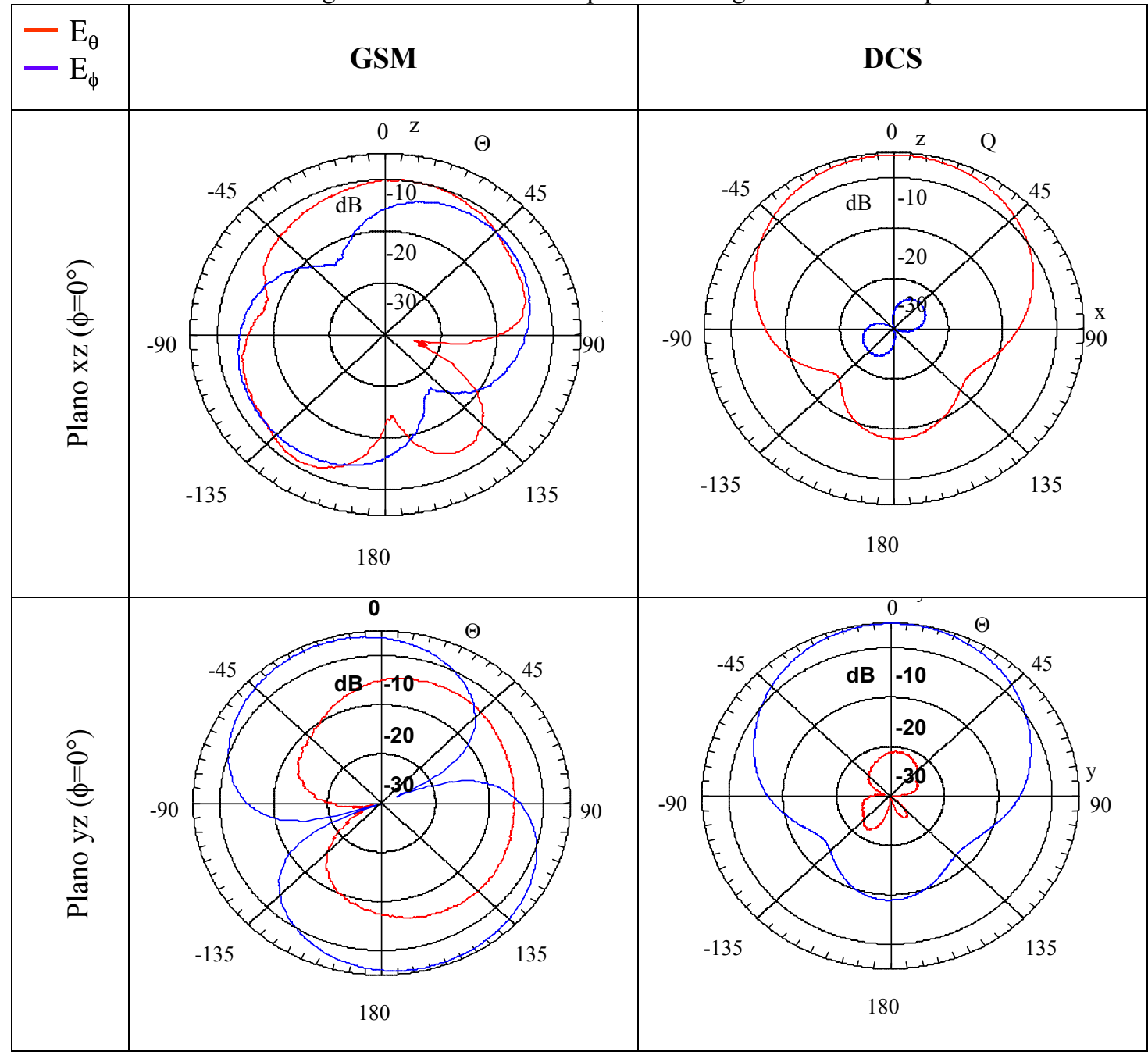


Otra ventaja de trabajar con antenas sin substrato dieléctrico reside en su facilidad de fabricación, y en la posibilidad de llevar a cabo ajustes hasta lograr las características de funcionamiento deseado. Ciertos elementos, como cortocircuitos, capacidades o diodos, cuya utilización conjuntamente con antenas impresas resulta complicada y costosa, pueden ser ahora introducidos con relativa facilidad.

Los diseños que se presentan en este capítulo están basados en las antenas planas en $\mathrm{F}$ invertida (Planar Inverted-F Antenna, PIFA). Las PIFAs son muy corrientes hoy en día en los terminales comerciales, ya que permiten operar en las bandas de frecuencias de los sistemas celulares, manteniendo un volumen reducido, puesto que su tamaño es aproximadamente un cuarto de la longitud de onda a la frecuencia de trabajo [5.32].

Uno de los problemas que se plantean al diseñar antenas para terminales móviles reside en cómo optimizar el uso de la potencia disponible [5.33], teniendo en cuenta que el espacio destinado a la antena es muy reducido. Esta limitación, junto con la distribución de los diferentes componentes del terminal, disminuye los grados de libertad en el diseño de una antena integrada. Hay que tener en cuenta además que el espacio disponible no se ajusta normalmente a una geometría rectangular, sino que suele estar adaptado a la forma de la cubierta externa, por lo que la antena adopta una forma tridimensional totalmente arbitraria. Desgraciadamente, estas configuraciones son muy difíciles de simular, puesto que sería necesario realizar una discretización muy fina de la estructura, lo que resulta costoso en tiempo de simulación. Así pues se suele recurrir a modelos simplificados en una primera aproximación.

\subsubsection{Estructura.}

La PIFA (Planar Inverted-F Antenna), cuya estructura se muestra en la Figura 5.12, puede estudiarse desde dos puntos de vista [5.32]: partiendo de una antena de hilo o de una antena impresa.

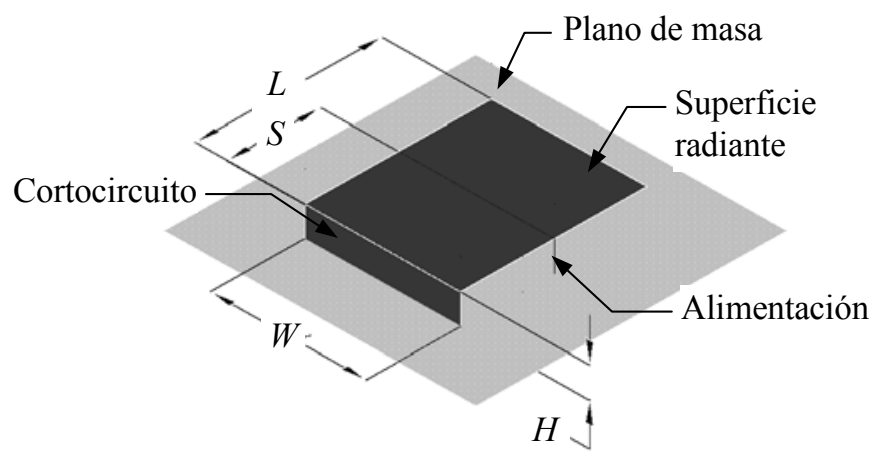

Figura 5.12: Estructura genérica de una PIFA.

En primer lugar, se puede considerar como una extensión del las antenas en F invertida (Inverted-F Antenna, IFA), en las que el hilo radiante se ha substituido por un plano metálico [5.32]. Con ello se consigue aumentar el ancho de banda de la estructura. 
Por otra parte, la PIFA se puede considerar como un caso particular de antena microtira cortocircuitada (Short-circuited Microstrip Antena, MSA). Ésta consiste en una antena impresa rectangular, cuyo modo resonante fundamental es el $\mathrm{TM}_{10}$. El tamaño del parche se puede reducir a la mitad situando un plano de cortocircuito entre el elemento radiante y el plano de masa siguiendo la línea en la que el campo eléctrico del modo fundamental es nulo, tal y como se muestra en la Figura 5.13. Con ello se reduce la longitud del parche de $\lambda / 2$ a $\lambda / 4$ sin que as características de funcionamiento se vean significativamente afectadas.

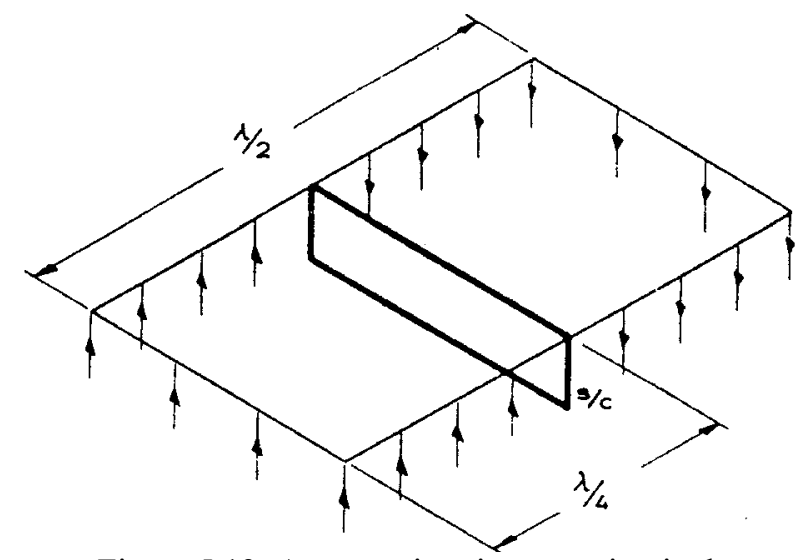

Figura 5.13: Antena microtira cortocircuitada.

Si el tamaño del cortocircuito es menor que el ancho del parche radiante, como en la Figura 5.14, la inductancia efectiva de la antena aumenta, y la frecuencia de resonancia es menor que la de una MSA cortocircuitada con las mismas dimensiones.

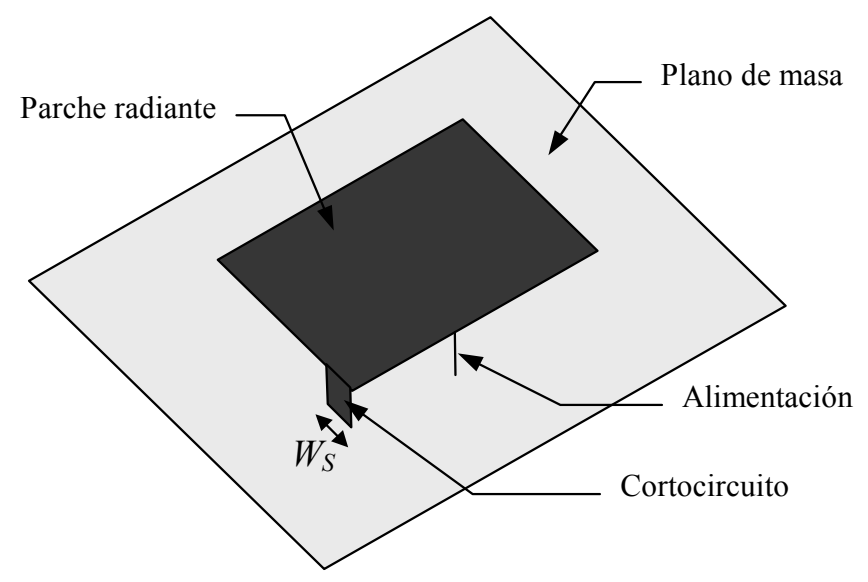

Figura 5.14: Estructura de una PIFA con cortocircuito menor que el ancho del parche.

\subsubsection{Distribución de campo y de corriente.}

Tanto el tamaño del cortocircuito como la relación de aspecto entre los lados del parche son importantes para determinar las características de funcionamiento de la antena: frecuencia de resonancia, ancho de banda, distribución de corriente en las superficies metálicas (parche y plano de masa) y campos radiados. 
En la Figura 5.15 [5.32] se ilustra la distribución de las componentes del campo eléctrico para una PIFA cuadrada $(\mathrm{L}=\mathrm{W})$ de altura $\mathrm{H}=\mathrm{L} / 4$ para diferentes tamaños del cortocircuito. Se puede observar cómo la componente en z se anula en la posición del cortocircuito. Por otra parte, cuando el cortocircuito se extiende a todo el lado del parche $\left(\mathrm{W}_{\mathrm{s}}=\mathrm{W}\right)$, la distribución de campos corresponde a la del modo fundamental $\mathrm{TM}_{10}$ de un parche rectangular convencional. Si se disminuye el tamaño del cortocircuito, aparecen componentes en x e y en los bordes en circuito abierto del parche, que contribuyen a la radiación de la PIFA.

En la Figura 5.16 [5.32] se muestra cómo afectan el tamaño del cortocircuito y la relación de aspecto del parche a la distribución de corrientes en la cara inferior del parche a la frecuencia de resonancia. Se puede apreciar cómo, para $\mathrm{W}-\mathrm{W}_{\mathrm{S}}<\mathrm{L}$, la corriente fluye hacia el lado en circuito abierto, el lado más largo del parche.

Por el contrario, para $\mathrm{W}-\mathrm{W}_{\mathrm{S}}>\mathrm{L}$, la corriente fluye hacia el circuito abierto en el lado estrecho del parche. Al disminuir el tamaño del cortocircuito, la longitud efectiva que debe recorrer la corriente es mayor, con lo que se logra disminuir la frecuencia de resonancia de la antena, manteniendo el tamaño constante.

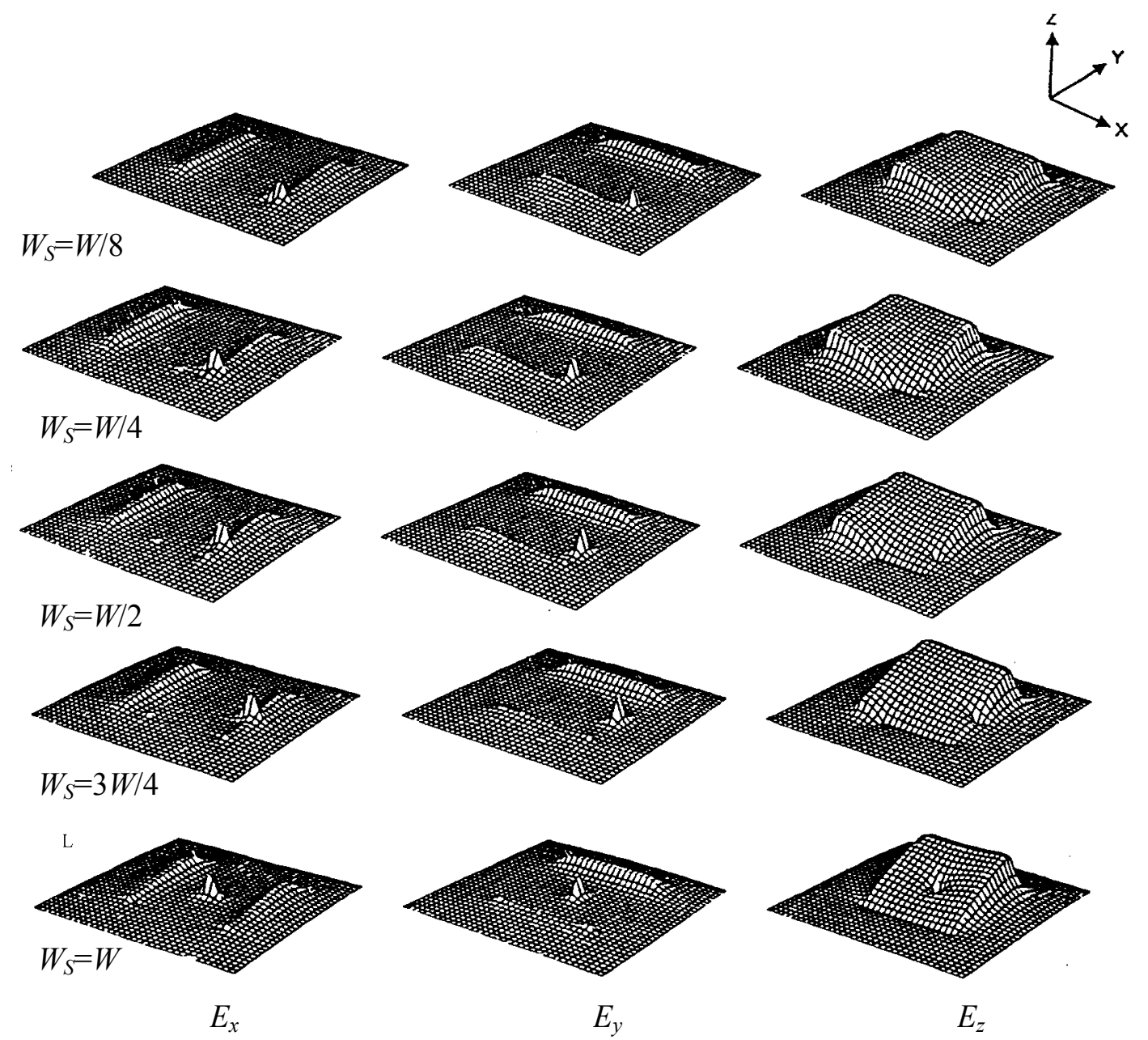

Figura 5.15: Distribución de las componentes de campo eléctrico para diferentes anchos del cortocircuito, con $\mathrm{W}=\mathrm{L}$. 


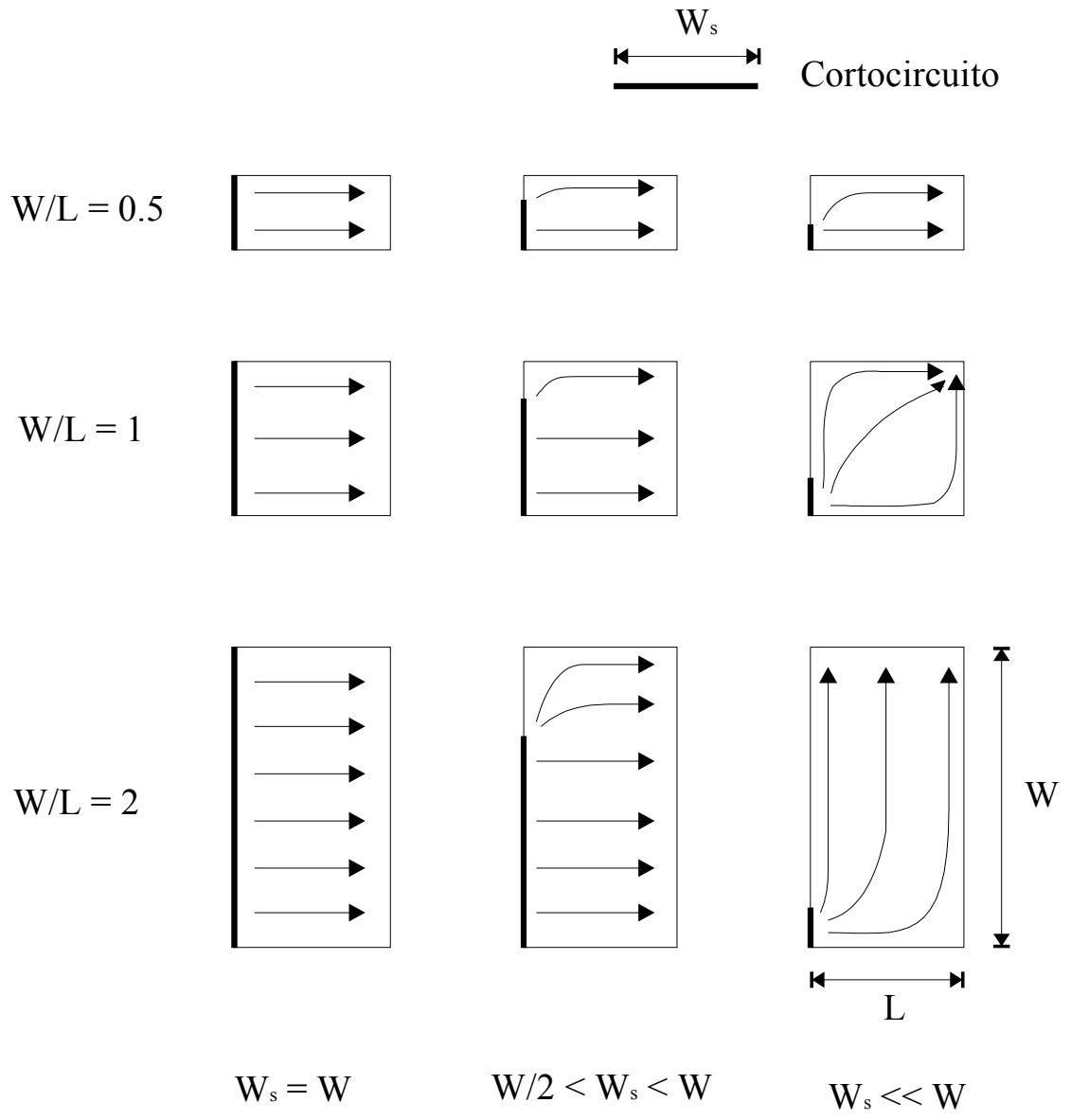

Figura 5.16: Distribución superficial de corriente en una PIFA para diferentes ratios $\mathrm{W} / \mathrm{L}$ y diferentes anchos del cortocircuito.

\subsubsection{Frecuencia de resonancia.}

Tal y como se ha mencionado en el apartado anterior, la frecuencia de resonancia de una PIFA disminuye al hacer más pequeño el cortocircuito. Una descripción detallada de cómo evoluciona la frecuencia de resonancia se puede encontrar en [5.32], incluyendo la gráfica de la Figura 5.17.

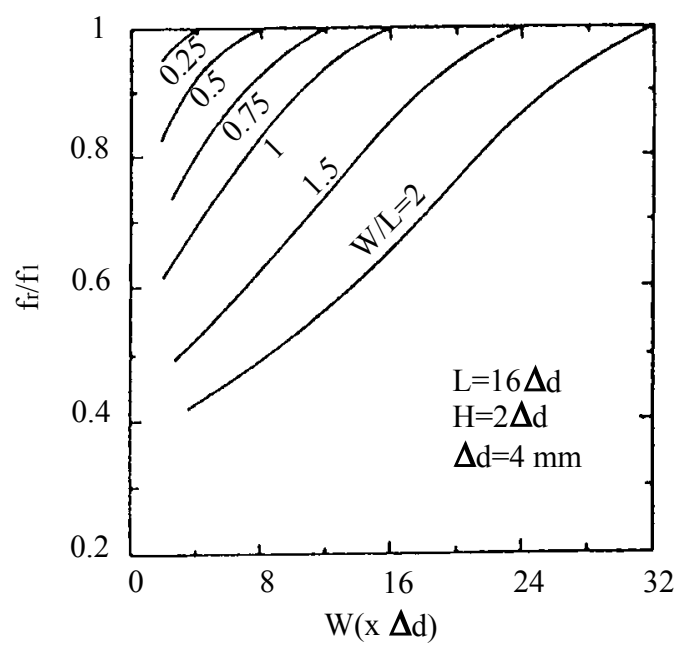

Figura 5.17: Frecuencia de resonancia de una PIFA en función del tamaño del cortocircuito, $\mathrm{W}_{\mathrm{S}}$. 
En una primera aproximación, se puede obtener la frecuencia de resonancia de una PIFA a partir de la longitud de sus lados como:

$$
\mathrm{W}+\mathrm{L}=\frac{\lambda}{4}
$$

En esta expresión no se tiene en cuenta el tamaño del cortocircuito. Por otra parte, a la vista de la distribución de corriente en la superficie del parche, se puede deducir que la longitud efectiva de la antena, a la frecuencia de resonancia, está determinada por el flujo de corriente sobre el parche y el cortocircuito. Así, cuando éste ocupa todo el lado del parche $\left(\mathrm{W}_{\mathrm{s}}=\mathrm{W}\right)$, se puede aplicar la fórmula de diseño de la IFA:

$$
\mathrm{W}+\mathrm{H}=\frac{\lambda}{4}
$$

Donde $\mathrm{H}$ representa la altura de la antena sobre el plano de masa.

El otro caso extremo se da cuando el anche del cortocircuito es prácticamente nulo $\left(\mathrm{W}_{\mathrm{S}} \approx 0\right)$. La corriente fluye entonces a lo largo de los bordes del parche, y la frecuencia de resonancia viene determinada por:

$$
\mathrm{W}+\mathrm{L}+\mathrm{H}=\frac{\lambda}{4}
$$

Si la altura del parche sobre el plano de masa es muy pequeña en términos de la longitud de onda, se puede despreciar el efecto de los campos evanescentes en el borde en circuito abierto, y los resultados obtenidos aplicando las ecuaciones (5.16) y (5.17) se corresponden con las medidas. Para los casos intermedios, con $0<\mathrm{W}_{\mathrm{S}} / \mathrm{W}<1$, la frecuencia de resonancia se puede obtener como:

$$
\begin{cases}\mathrm{f}_{\mathrm{r}}=\mathrm{r} \cdot \mathrm{f}_{1}+(1-\mathrm{r}) \cdot \mathrm{f}_{2}, & \frac{\mathrm{W}}{\mathrm{L}} \leq 1 \\ \mathrm{f}_{\mathrm{r}}=\mathrm{r}^{\mathrm{k}} \cdot \mathrm{f}_{1}+\left(1-\mathrm{r}^{\mathrm{k}}\right) \cdot \mathrm{f}_{2}, & \frac{\mathrm{W}}{\mathrm{L}} \geq 1\end{cases}
$$

Con:

$$
\begin{aligned}
& \mathrm{r}=\frac{\mathrm{W}_{\mathrm{S}}}{\mathrm{W}} \\
& \mathrm{k}=\frac{\mathrm{W}}{\mathrm{L}}
\end{aligned}
$$

Siendo $\mathrm{f}_{1}$ la frecuencia obtenida aplicando la expresión (5.16), $\mathrm{y}_{2}$ la deducida de:

$$
\mathrm{W}+\mathrm{L}+\mathrm{H}-\mathrm{W}_{\mathrm{S}}=\frac{\lambda}{4}
$$




\subsubsection{Ancho de banda.}

El ancho de banda de una PIFA depende principalmente de tres factores: la altura del parche sobre el plano de masa, la relación de aspecto W/L, y el tamaño del cortocircuito. Tal y como se puede observar en la Figura 5.18 [5.32], para $\mathrm{W}_{\mathrm{S}}=\mathrm{W}$, el ancho de banda relativo de la antena aumenta al incrementarse $\mathrm{W} / \mathrm{L}$.

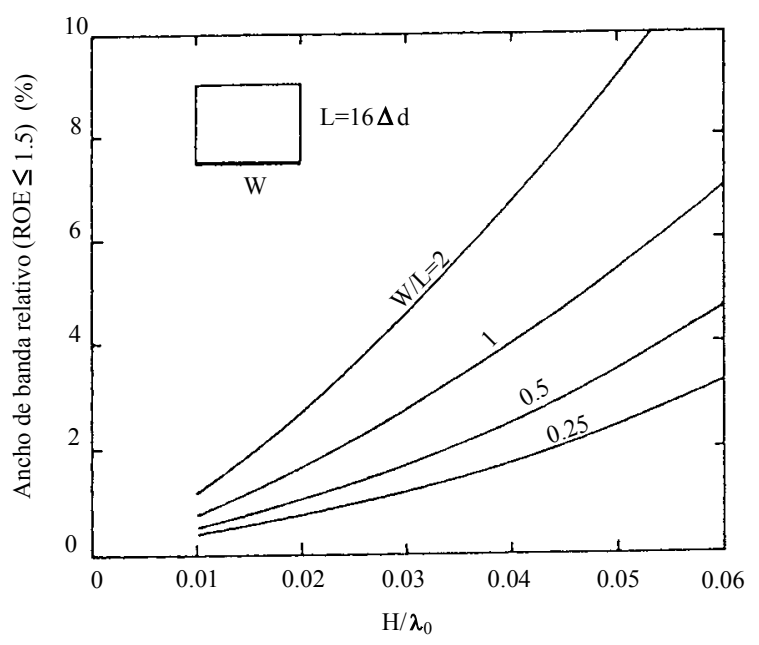

Figura 5.18: Ancho de banda de la PIFA en función de la altura del parche, para $\mathrm{W}_{\mathrm{S}}=\mathrm{W}$ y diferentes relaciones de aspecto $\mathrm{W} / \mathrm{L}$.

Del mismo modo, para una relación de aspecto fija, el ancho de banda aumenta con la altura y con el tamaño del cortocircuito, tal y como se muestra en la Figura 5.19 [5.32].

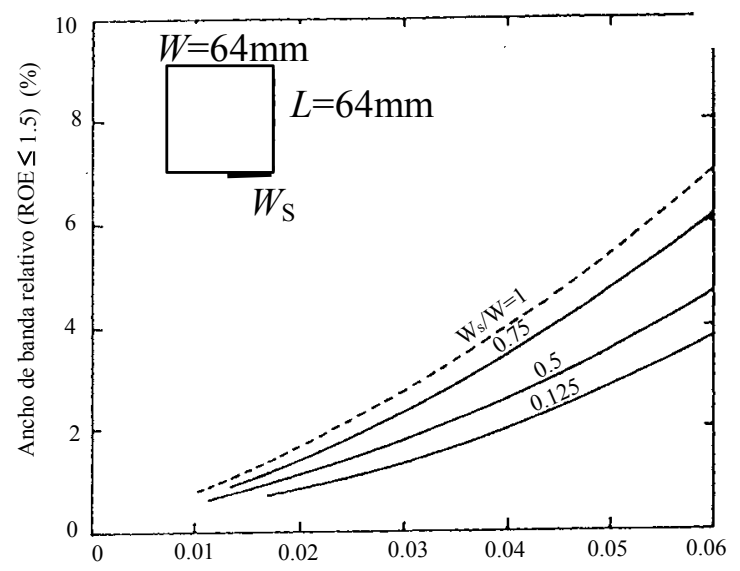

Figura 5.19: Ancho de banda de una PIFA para diferentes valores de $\mathrm{W}_{\mathrm{S}} / \mathrm{L}$, con $\mathrm{W}=\mathrm{L}$.

Hasta ahora se ha considerado que la PIFA estaba situada sobre un plano de masa de dimensiones infinitas. En los casos prácticos el plano de masa tiene un tamaño limitado. En el caso de antenas integradas en terminales móviles, éste es muy reducido. Resulta entonces complicado determinar a priori las características de radiación o el ancho de banda máximo que se puede alcanzar. Sin embargo, en general el ancho de banda de una PIFA sobre terminal es mayor que en el caso de la antena sobre un plano de masa infinito, lo que favorece su utilización en aplicaciones móviles. 


\subsection{PIFA CON FILTRO ESPOLÓN.}

\subsubsection{Descripción del problema.}

El objetivo perseguido es diseñar una antena de doble banda para GSM y DCS, con un tamaño máximo de $40 \mathrm{~mm}$ x $26 \mathrm{~mm}$ x $8 \mathrm{~mm}$. Para ello se define un terminal tipo como el mostrado en la Figura 5.20.

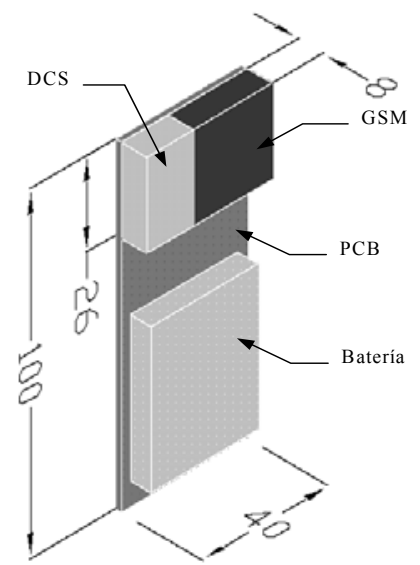

Figura 5.20: Descripción del problema típico.

En el Capítulo 3 se comentaba que una de las limitaciones en el diseño de antenas reside en su tamaño, estableciendo la relación entre el volumen de una antena inscrita en la esfera de radio $\mathrm{a}=1 / \mathrm{k}=1 / 2 \pi$ descrita en la Figura 5.21 (a) y su factor de calidad mínimo y su ancho de banda máximo [5.34]. Sin embargo, para ello se asume que la antena radia en el espacio libre, lo que no es exacto en el caso de las antenas utilizadas en terminales de comunicaciones móviles [5.35]. En estos casos, el radio a es lo suficientemente grande como para llegar a incluir todo el terminal, tal y como se muestra en la Figura 5.21 (b) y (c).

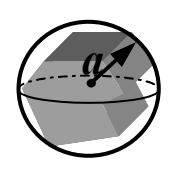

(a)

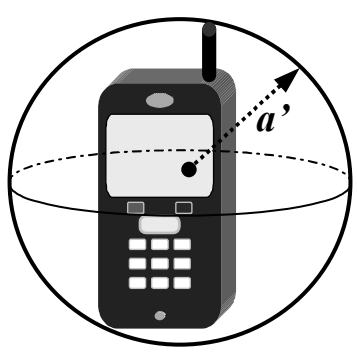

(b)

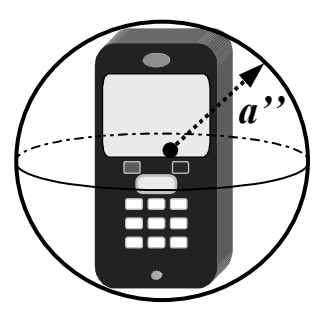

(c)

Figura 5.21: Representación gráfica de la esfera definida por McLean.

(a) Antena en el espacio libre.

(b) Terminal con antena externa.

(c) Terminal con antena interna.

El efecto de la pletina debe pues ser tenido en cuenta al analizar el funcionamiento de la antena [5.32], ya que la radiación electromagnética no es debida únicamente a la distribución de corrientes en el parche, sino que las corrientes inducidas el plano de masa contribuyen también a la 
distribución de campos. Así pues el tamaño del plano de masa juega un papel importante en lo relativo al ancho de banda, las frecuencias de resonancia y los diagramas de radiación [5.36].

\subsubsection{Antena dual con filtro espolón.}

En el apartado 5.3 se describía cómo la inserción de un filtro espolón permitía obtener una operación dual para antenas impresas. Sin embargo, el ancho de banda que se alcanzaba era insuficiente para cumplir con los estándares de comunicaciones móviles. Por otra parte, se ha descrito en el apartado anterior cómo la utilización de PIFAs permite obtener frecuencias de resonancia bajas en un volumen de espacio reducido. Combinando ambas técnicas, se ha desarrollado una antena dual de pequeño tamaño, capaz de operar simultáneamente en dos bandas de frecuencias distintas [5.37]-[5.38].

Esta antena, representada en la Figura 5.22, consiste en un parche rectangular situado a $h=8 \mathrm{~mm}$ del plano de masa. Para lograr una estructura similar a una PIFA se cortocircuita la estructura mediante un pin metálico en una de sus esquinas. La alimentación, por sonda coaxial, se conecta al borde superior del parche. Las dimensiones de la antena se calculan para obtener una resonancia a la frecuencia de 1,8 GHz. En el perímetro del parche conductor se introduce el filtro espolón, cuya longitud se ajusta para introducir una nueva resonancia a $925 \mathrm{MHz}$.

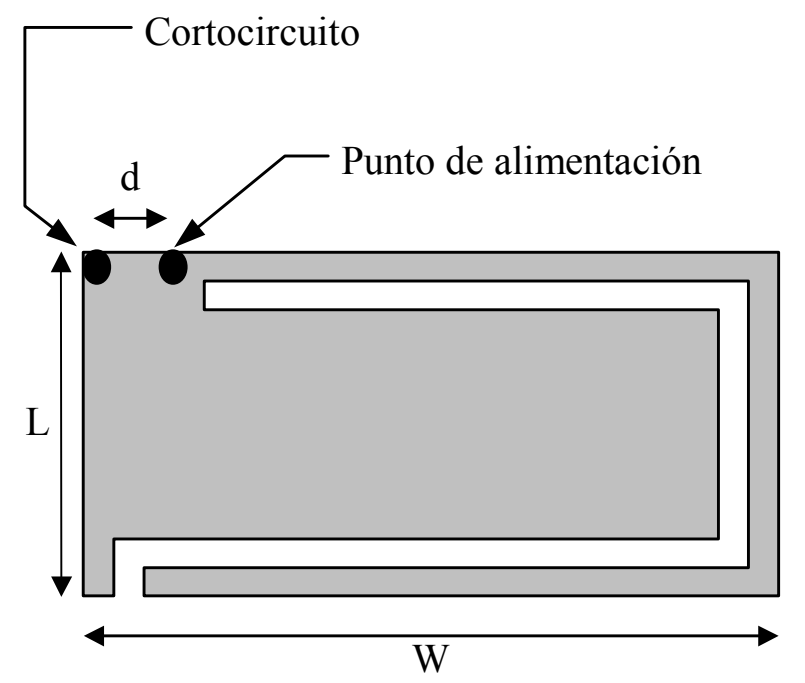

Figura 5.22: PIFA rectangular con filtro espolón, $W=38 \mathrm{~mm}$, $\mathrm{L}=13 \mathrm{~mm}, \mathrm{~h}=8 \mathrm{~mm}, \mathrm{~d}=5 \mathrm{~mm}$.

Las dimensiones totales de la antena para cubrir las bandas de GSM y DCS son $\mathrm{L}=38 \mathrm{~mm}$ y $\mathrm{W}=13 \mathrm{~mm}$. La antena es por lo tanto lo suficientemente pequeña como para poder ser integrada en la mayoría de los terminales móviles. El punto de alimentación se coloca en el borde superior del parche para obtener el máximo de ancho de banda [5.39]. Accesoriamente, se pueden ajustar las frecuencias de resonancia variando la distancia d entre el punto de alimentación y el cortocircuito.

Para analizar el comportamiento de la antena, tanto en las simulaciones como para las medidas, se 
consideró que estaba situada sobre un plano de masa de 40mm x 100mm. También la influencia de la batería del terminal se deberá tener en cuenta, tal y como se mostrará en apartados posteriores. La estructura se muestra en la Figura 5.23, y su implementación en la Figura 5.24..

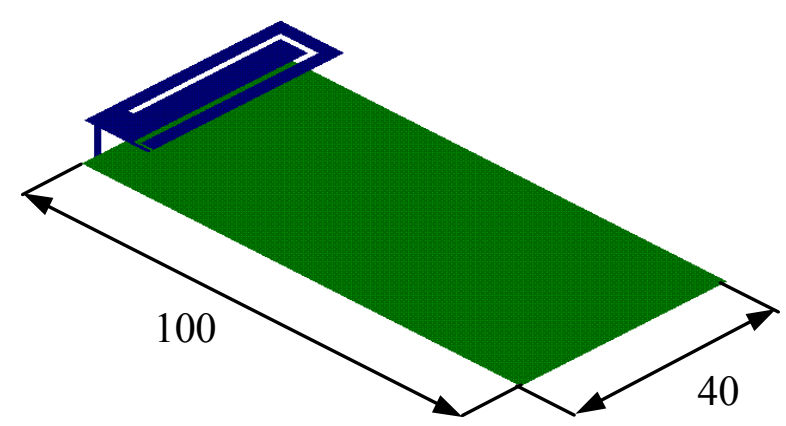

Figura 5.23: Antena sobre el soporte metálico que simula el terminal.

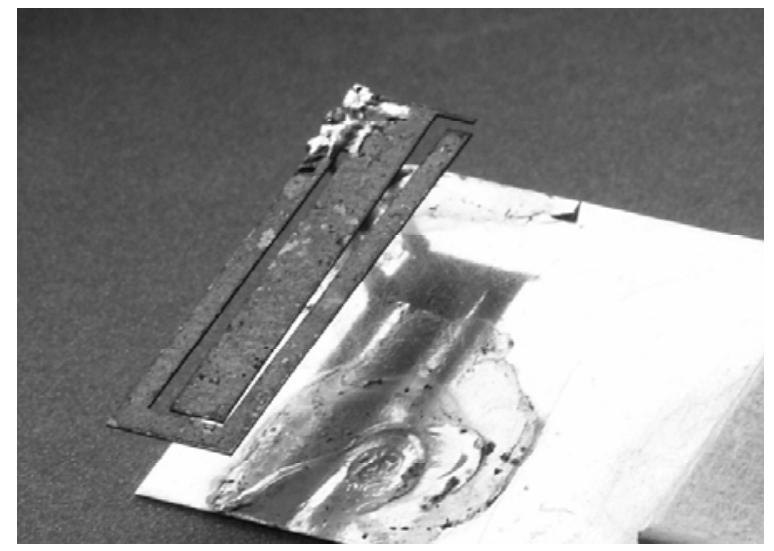

Figura 5.24: PIFA con filtro espolón.

En la Figura 5.25 se muestra una comparación entre los resultados obtenidos por simulación y las medidas efectuadas sobre un prototipo. Para estudiar el comportamiento teórico de la estructura se utilizó el simulador electromagnético CONCEPT, descrito en el Capítulo 4. Las medidas se llevaron a cabo utilizando un analizador de redes HP8719D. Los resultados obtenidos en ambos casos son similares.

El ancho de banda para frecuencias de GSM, medido para unas pérdidas de retorno $\mathrm{S}_{11}$ inferiores a $-10 \mathrm{~dB}(\mathrm{ROE}<2)$, es de $26 \mathrm{MHz}$ para una frecuencia central de $970 \mathrm{MHz}$, lo que corresponde a un ancho de banda relativo del 2,68\%. Para DCS se alcanza un ancho de banda relativo del 8,58\%, correspondiente a $152 \mathrm{MHz}$ para una frecuencia central de 1,77 GHz. Sin embargo, en la actualidad los criterios utilizados son menos restrictivos, y a menudo se define el ancho de banda para una ROE inferior a 2,5 $\left(\mathrm{S}_{11}<-7,5 \mathrm{~dB}\right)$ o incluso $3\left(\mathrm{~S}_{11}<-6 \mathrm{~dB}\right)$, por lo que la antena podría cumplir las especificaciones dadas. 


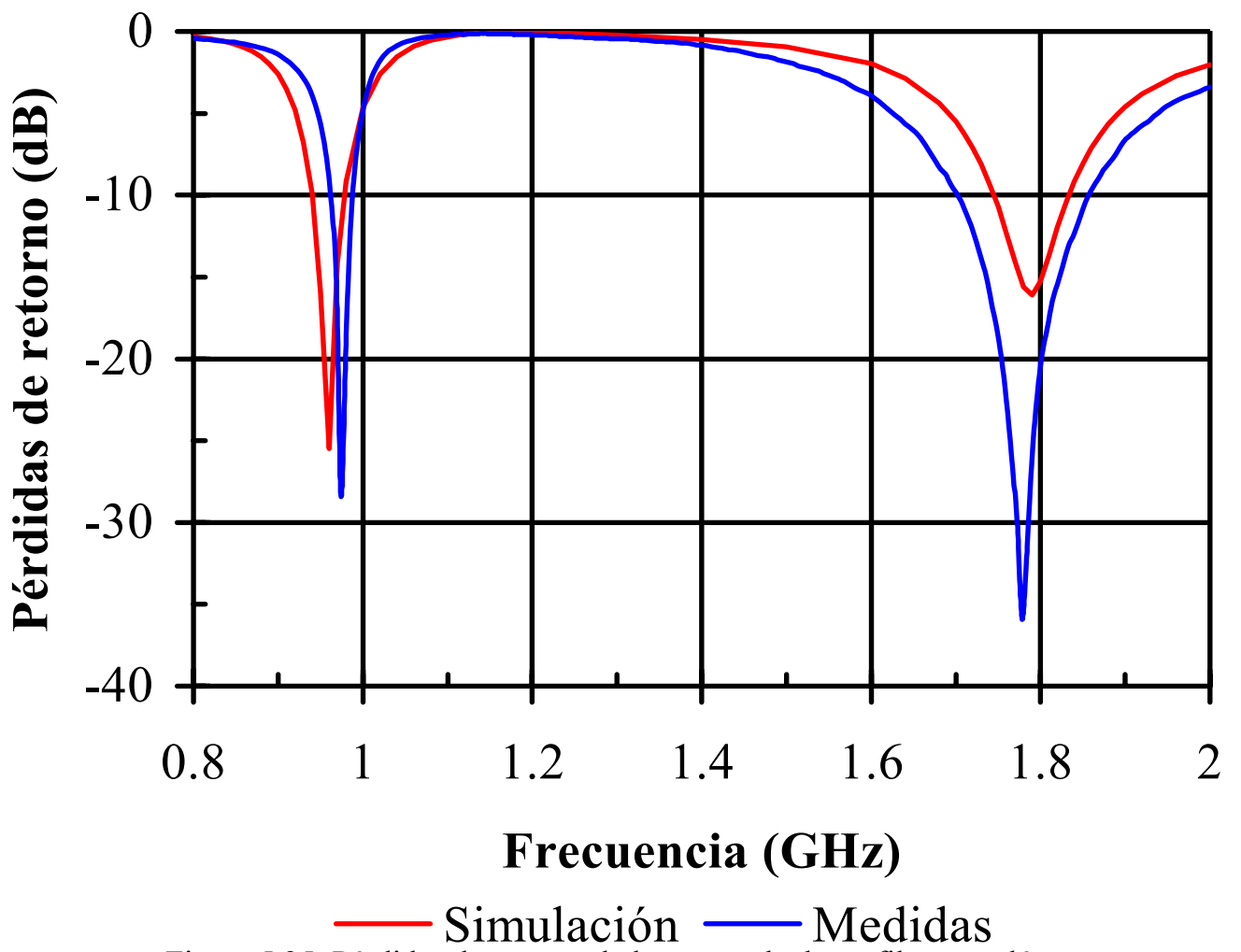

Figura 5.25: Pérdidas de retorno de la antena dual con filtro espolón.

En ambos casos, los resultados obtenidos estarían dentro de las especificaciones, si se tiene en cuenta que tanto las simulaciones como las medidas se llevaron a cabo para un terminal ficticio, sin cubierta de plástico. La presencia de ésta se traduciría en una disminución de las frecuencias de resonancia.

En la Figura 5.26 y la Figura 5.27 se muestran las distribuciones de campo eléctrico y magnético en un plano cercano a la superficie de la antena, para las bandas de frecuencias de GSM y DCS, respectivamente. En ellas se puede apreciar cómo la parte interior del parche es la zona activa cuando se opera en DCS, mientras que el filtro espolón, y la rama exterior del parche se activan a frecuencias de GSM.
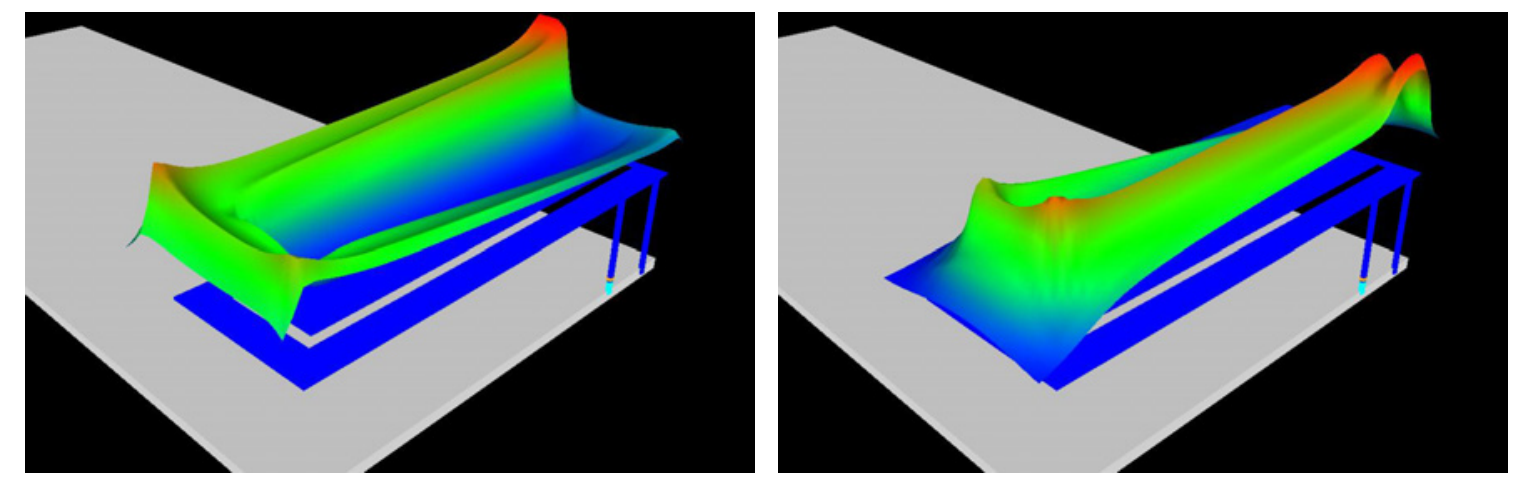

Figura 5.26: Distribución de campo eléctrico (izquierda) y magnético (derecha) en la banda de GSM. 

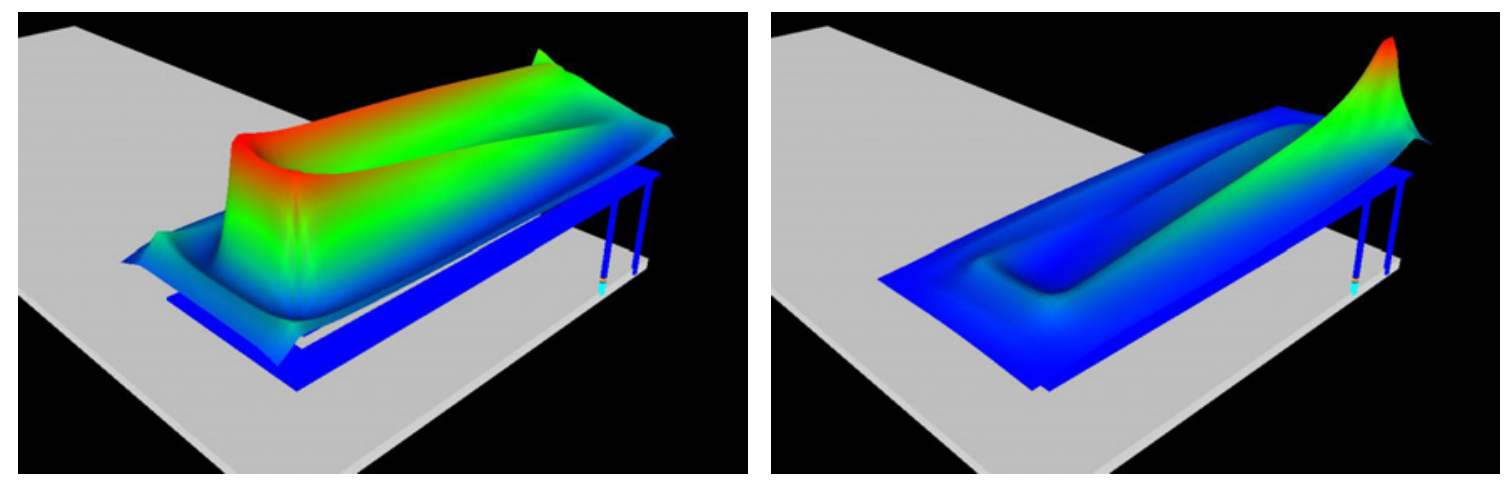

Figura 5.27: Distribución de campo eléctrico (izquierda) y magnético (derecha) en la banda de DCS.

La combinación del parche radiante con el filtro espolón da lugar a los diagramas de radiación de la Figura 5.28, en los que se observa el carácter casi omnidireccional de la antena en el plano horizontal, similar al comportamiento de un dipolo en $\lambda / 2$. Este efecto es sobre todo apreciable en la banda de GSM.
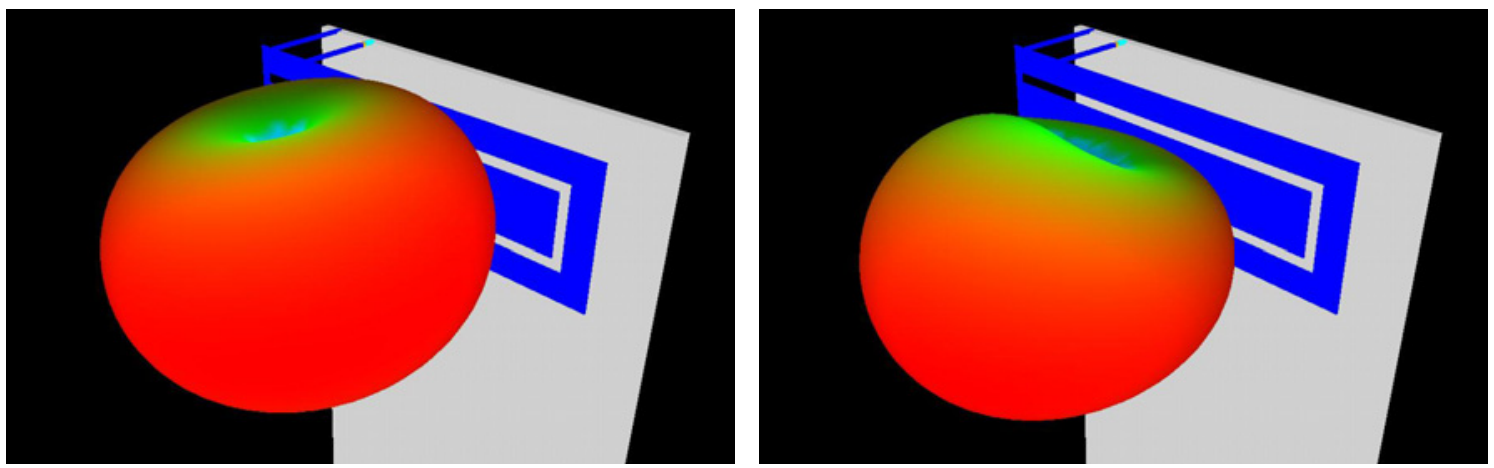

Figura 5.28: Diagrama de radiación en las bandas de GSM (izquierda) y DCS (derecha).

Se midieron igualmente los diagramas de radiación a cada una de las frecuencias de resonancia. La ganancia alcanzada es de -1,3 dBi en la banda de GSM, y 1,6 dBi en la de DCS. En la Tabla 5.6 se muestran los resultados obtenidos para los cortes en dos de los planos de principal interés, definidos según el sistema de coordenadas de la Figura 5.29.

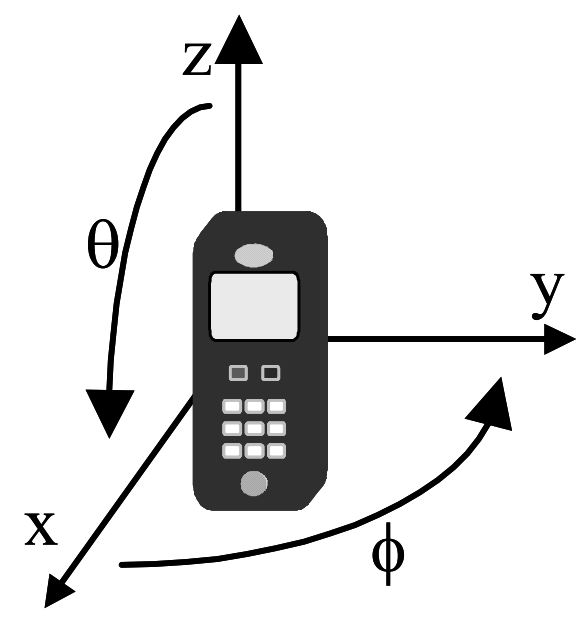

Figura 5.29: Sistema de coordenadas utilizado en las medidas de los diagramas de radiación. 
Tabla 5.6: Diagramas de radiación de la antena dual con filtro espolón.

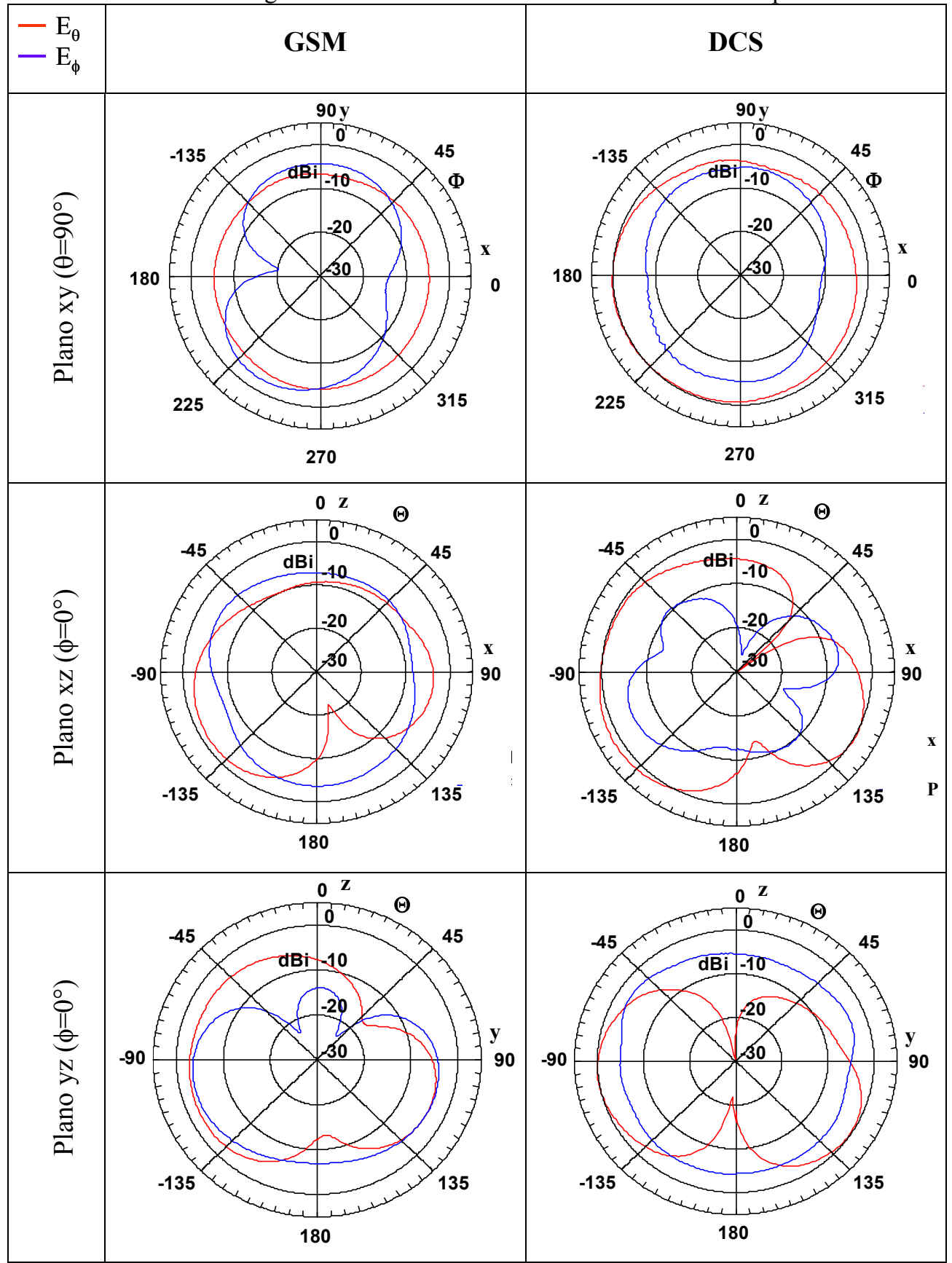

Se puede apreciar cómo se produce una inclinación del haz debido al efecto del plano de masa. Sin embargo, esto no supone ningún inconveniente para un terminal de comunicaciones móviles, puesto que su orientación espacial, en condiciones normales de uso, es totalmente aleatoria.

\subsubsection{Efecto del tamaño del terminal.}

Cuando se excita el parche para que funcione como antena, aparecerán corrientes inducidas en la superficie del plano de masa, también denominado pletina p PCB (Printed Circuit Board). Teniendo en cuenta que la antena es un elemento resonante, y que la longitud del terminal suele ser del orden de $\lambda / 2$, se puede aproximar el funcionamiento de la estructura por una combinación de 
resonadores en serie y en paralelo como la presentada en la Figura 5.30 [5.40].

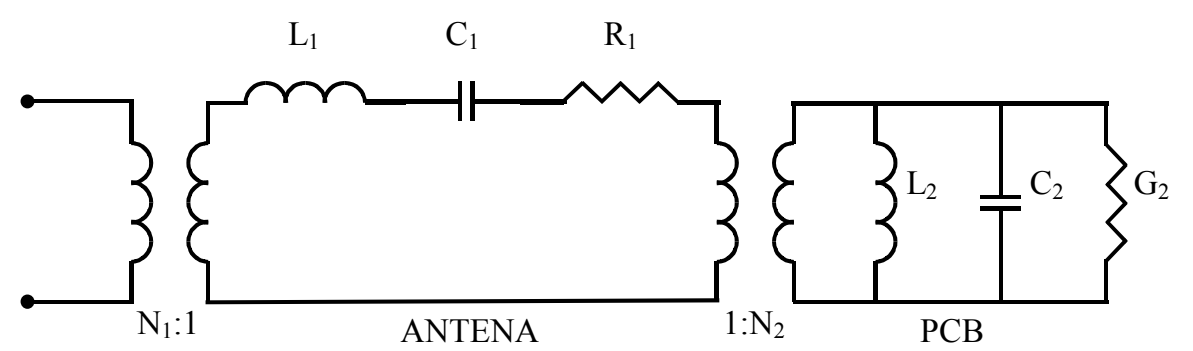

Figura 5.30: Circuito equivalente serie de una antena monobanda sobre un plano de masa.

Teniendo en cuenta que el comportamiento de una antena PIFA se asemeja más al de un circuito resonante paralelo, una situación más realista podría ser la descrita en la Figura 5.31.

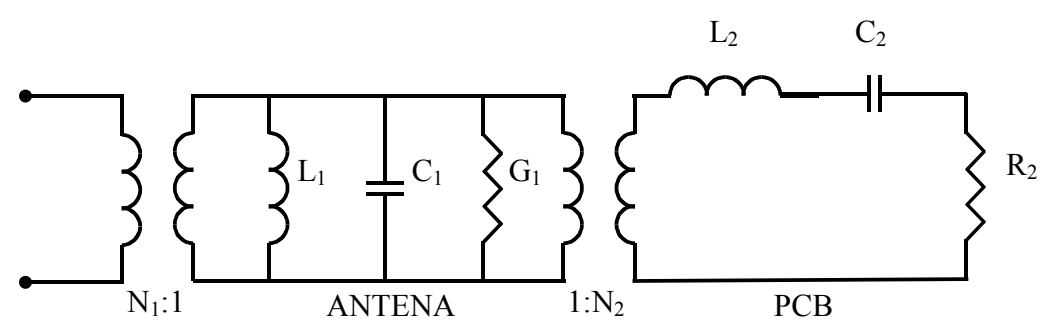

Figura 5.31: Circuito equivalente paralelo de una antena monobanda sobre un plano de masa.

En estos circuitos equivalentes, la frecuencia de resonancia del plano de masa estará determinada por su longitud física. Así, para analizar cómo la longitud del terminal afecta a las características de la antena, se estudió el caso de la antena de doble banda sobre un plano de masa de $40 \mathrm{~mm}$ de ancho y de longitud L, representada en la Figura 5.32.

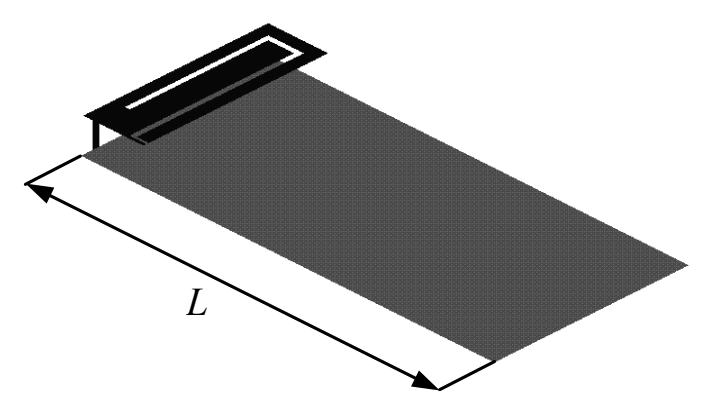

Figura 5.32: Estructura de doble banda sobre plano de masa.

En primer lugar, se pretende determinar el efecto de las variaciones de la longitud del PCB sobre la frecuencia de resonancia y el ancho de banda de la antena. Así pues, se llevaron a cabo simulaciones para diferentes valores de la longitud $L$ del plano de masa. En la Figura 5.33 se muestra cómo, para cada una de las bandas de frecuencias, la evolución es diferente. Estos cambios en la frecuencia de resonancia se deben a que las dimensiones del plano de masa son equiparables a un cuarto de la longitud de onda en frecuencias de GSM, y a media longitud de onda en DCS. El PCB contribuye pues de forma importante a la radiación del terminal, al actuar como un 'contrapolo' [5.41], [5.42]. 


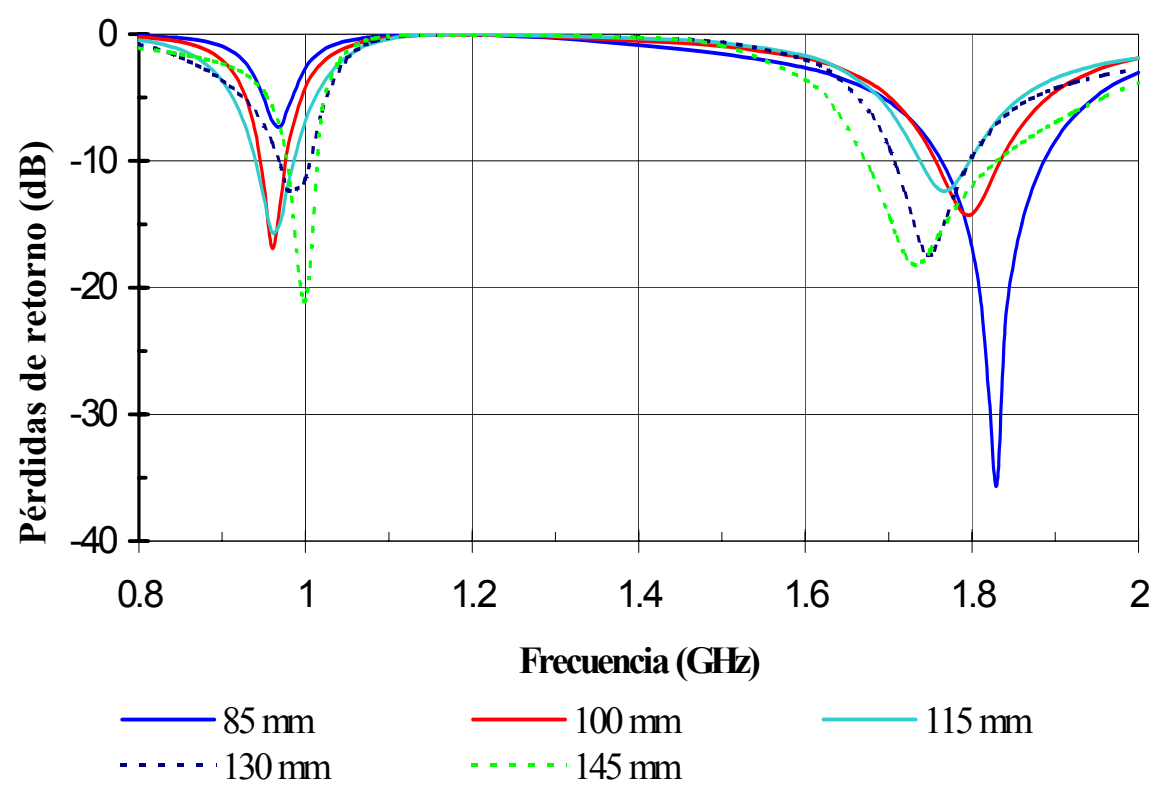

Figura 5.33: Pérdidas de retorno para diferentes longitudes del terminal (del PCB).

Otro aspecto importante que hay que tener en cuenta es la variación del ancho de banda de la antena debido al tamaño del PCB. En algunos casos se han llegado a obtener variaciones de hasta el $300 \%$ en el ancho de banda de un parche resonante en la banda de UMTS, ajustando la longitud del terminal [5.43]. Por otra parte, los resultados presentados en [5.44] y [5.45] indican que, para obtener resultados óptimos en lo referente al ancho de banda, la longitud del PCB debería ser igual a $0,38 \lambda_{0}$, siendo $\lambda_{0}$ la longitud de onda a la frecuencia central de la banda considerada.

En la Figura 5.34 se puede apreciar la variación del ancho de banda, definido para unas pérdidas de retorno inferiores a $-6 \mathrm{~dB}$, en función de la longitud del plano de masa. De nuevo, el efecto es diferente para ambas bandas de frecuencias. El caso ideal sería un terminal de longitud tal que se obtuviera un buen compromiso para GSM y DCS. Sin embargo el tamaño del terminal está determinado por criterios alejados de las necesidades de diseño de la antena.

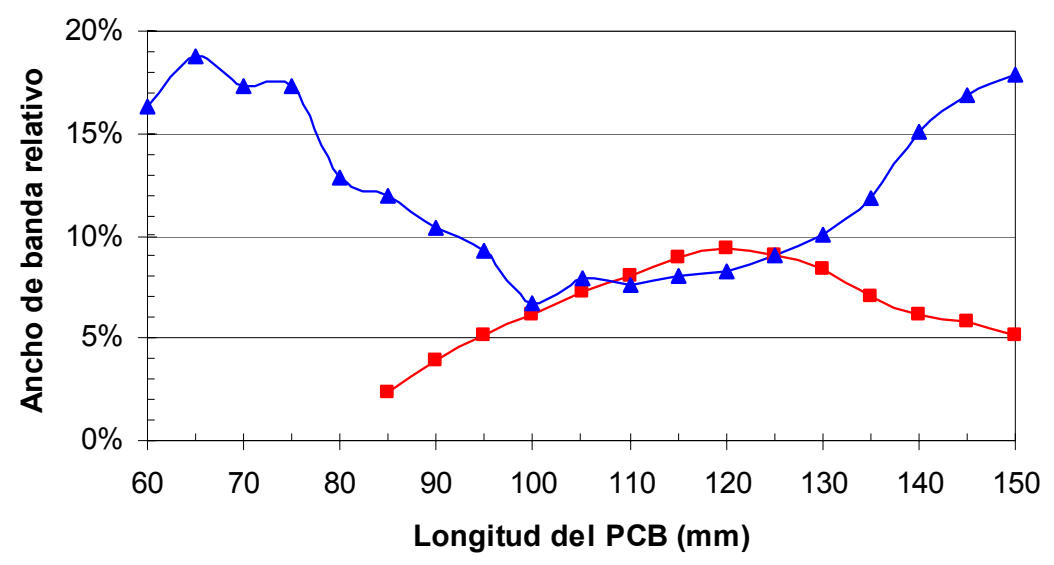

$\rightarrow$ GSM $\rightarrow$ DCS

Figura 5.34: Ancho de banda en función del tamaño del terminal para las bandas de frecuencias de GSM y DCS. 
Tal y como se puede apreciar en la Figura 5.34, resulta más fácil a priori cumplir las especificaciones de ancho de banda para DCS. Sin embargo, el tamaño típico de los teléfonos móviles se sitúa entre 95 y $110 \mathrm{~mm}$ de longitud. Para pletinas de este tamaño se obtienen los peores resultados en cuanto a ancho de banda para frecuencias de DCS. Por lo tanto es necesario encontrar soluciones que permitan optimizar dicho ancho de banda.

Este efecto es evidente cuando se consideran las distribuciones de campo eléctrico en un corte perpendicular al PCB del terminal. Así, en la Figura 5.35 se observa cómo, en el caso de GSM, al aumentar la longitud del terminal se altera la distribución de campos sobre el mismo, apareciendo una zona "fría" en la parte central del PCB.

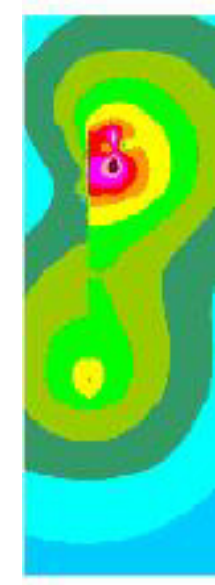

$\mathrm{L}=85 \mathrm{~mm}$

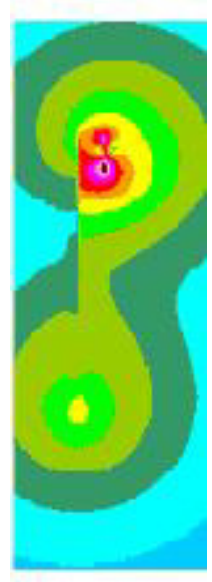

$\mathrm{L}=100 \mathrm{~mm}$

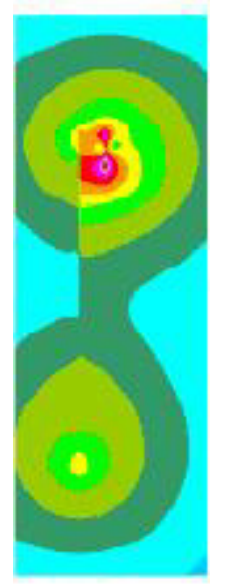

$\mathrm{L}=115 \mathrm{~mm}$

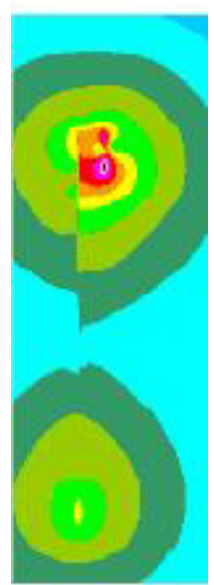

$\mathrm{L}=130 \mathrm{~mm}$

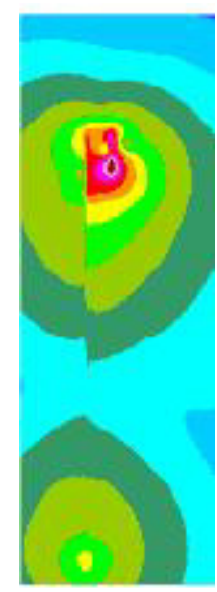

$\mathrm{L}=145 \mathrm{~mm}$
$\mathrm{dbV} / \mathrm{m}$

85-90

80-85

75-80

-70-75

65-70

60-65

55-60

50-55

45-50

40-45

35-40

30-35

- 25-30

Figura 5.35: Distribución de campo eléctrico para diferentes longitudes del PCB en la banda de GSM.

En la Figura 5.36, por el contrario, se ilustra el efecto de alargar el terminal para frecuencias de DCS. En este caso, la frecuencia de trabajo es más elevada, el doble que en GSM. Así, el alargar el PCB puede provocar la aparición de un nuevo máximo en la zona central del terminal. Esto resulta perjudicial para su funcionamiento, puesto que provocaría un aumento de las pérdidas por absorción en la mano del usuario.

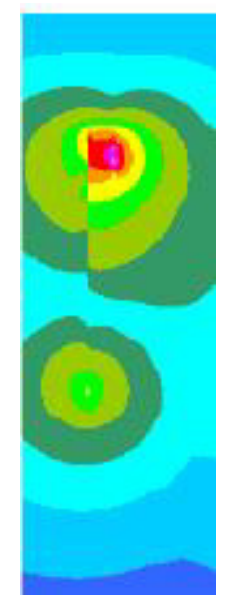

$\mathrm{L}=85 \mathrm{~mm}$

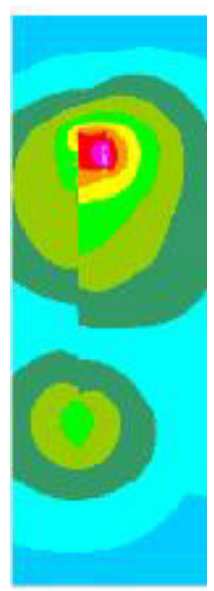

$\mathrm{L}=100 \mathrm{~mm}$

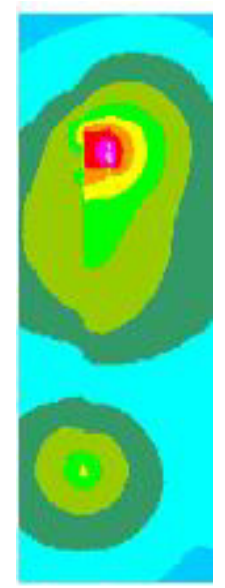

$\mathrm{L}=115 \mathrm{~mm}$

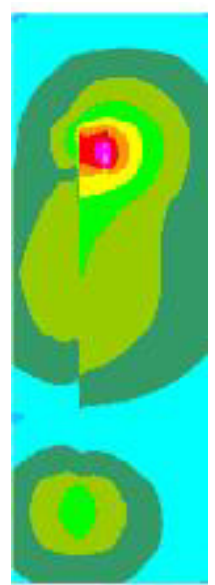

$\mathrm{L}=130 \mathrm{~mm}$

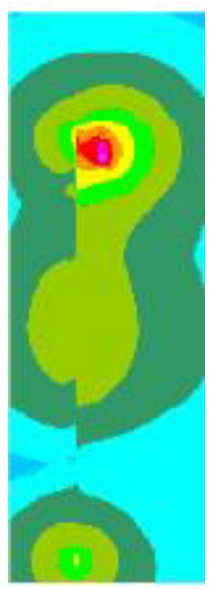

$\mathrm{L}=145 \mathrm{~mm}$
$\mathrm{dbV} / \mathbf{m}$

85-90

$\square$ 80-85

$75-80$

$70-75$

$60-65$

$55-60$

50-55

$45-50$

40-45

$\square-45$

30-35

- 25-30

Figura 5.36: Distribución de campo eléctrico para diferentes longitudes del PCB en la banda de DCS. 


\subsubsection{Influencia de la batería.}

Además del tamaño total del terminal, existen otros aspectos que condicionan las características de adaptación y radiación de una antena integrada. Los diferentes componentes que forman el móvil afectarán al comportamiento global del sistema. Así pues es necesario tener en cuenta en el diseño elementos como por ejemplo las cubiertas de apantallamiento de los circuitos de RF ( $R F$ shields), el motor del vibrador o la batería. El efecto de esta última se estudia en este apartado.

Tanto la presencia de la batería en sí, como su posición en el terminal influyen en la distribución superficial de corrientes, y por lo tanto en las características de adaptación y de radiación de la antena. Para representarla se recurrió a una caja metálica de $50 \mathrm{~mm}$ x $36 \mathrm{~mm} \times 7 \mathrm{~mm}$, soldada al plano de masa. El nuevo modelo del terminal se muestra en la Figura 5.37. El parámetro $d$ representa la distancia de separación entre la antena y la batería.

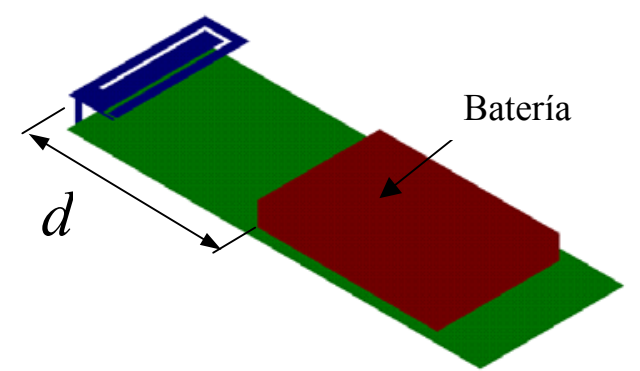

Figura 5.37: Modelo del terminal con batería.

Al situar la batería sobre el terminal aparece un acoplamiento entre ésta y el parche radiante, que se refleja en cambios en las características de radiación y de adaptación del sistema. Así, en la Figura 5.38 se muestra cómo varían las pérdidas de retorno en función de la posición de la batería con respecto a la antena.

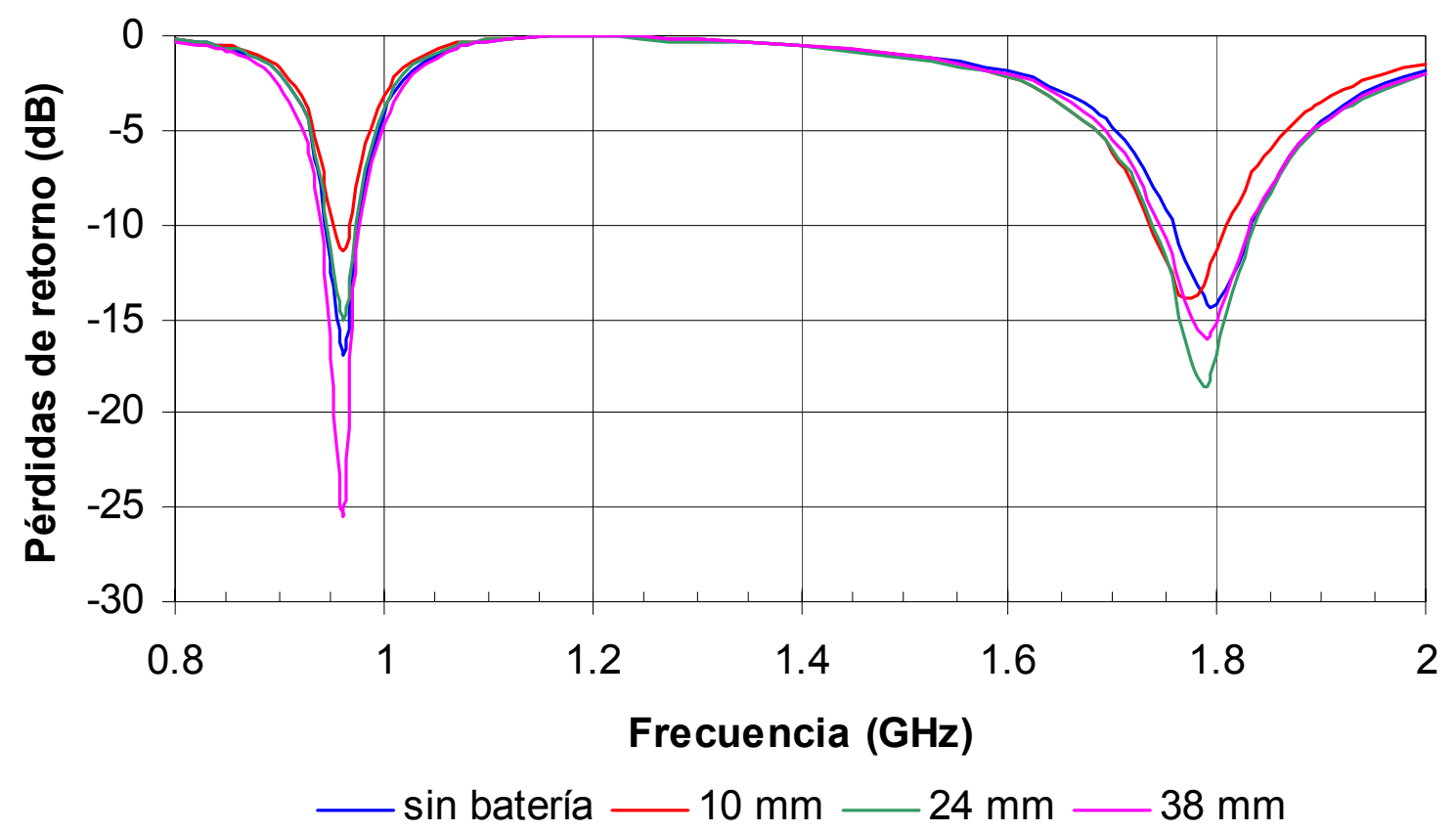

Figura 5.38: Pérdidas de retorno simuladas en función de la posición de la batería. 
Se puede observar cómo el efecto de la posición de la batería es distinto para las bandas de GSM y DCS. En GSM la presencia de la batería se traduce en la aparición de un efecto capacitivo, que no afecta a la frecuencia de resonancia pero sí al valor de las pérdidas de retorno. En DCS sin embargo se puede producir una desintonización de la frecuencia de resonancia de la antena, que habrá que compensar modificando las dimensiones del parche y del filtro.

La presencia de la batería influye igualmente sobre el ancho de banda, tal y como se muestra en la Figura 5.39. Sin embargo esta influencia no es tan significativa si se compara con el efecto del tamaño del terminal.

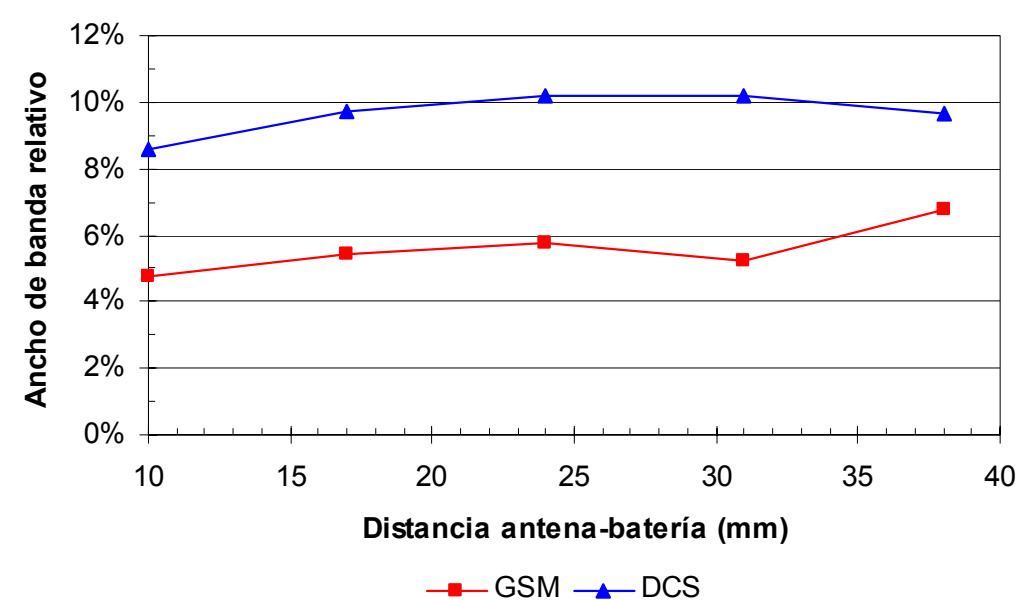

Figura 5.39: Ancho de banda relativo en función de la distancia de la antena a la batería.

La presencia de la batería repercute asimismo en la distribución de los campos en las inmediaciones del terminal. En la Figura 5.40 se muestra la distribución del campo eléctrico para la banda de GSM en un plano perpendicular a la pletina del terminal. Se puede apreciar cómo este efecto no es especialmente significativo en la banda de GSM.

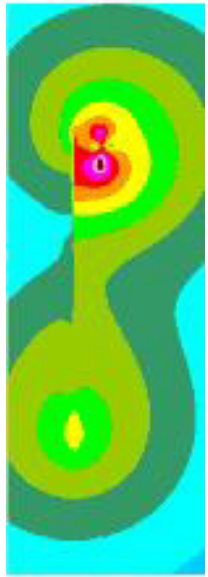

Sin batería

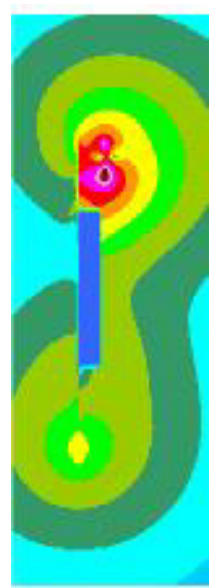

$\mathrm{d}=10 \mathrm{~mm}$

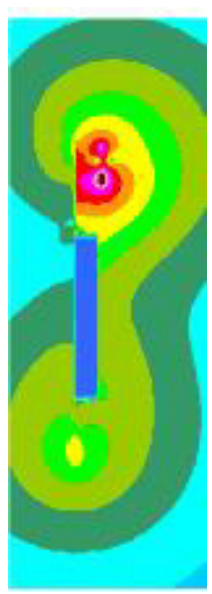

$\mathrm{d}=17 \mathrm{~mm}$

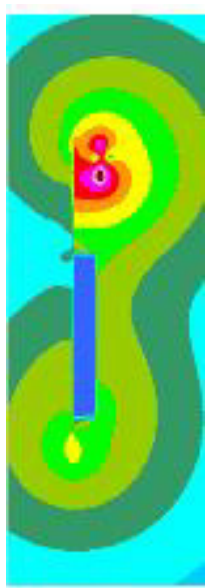

$\mathrm{d}=24 \mathrm{~mm}$

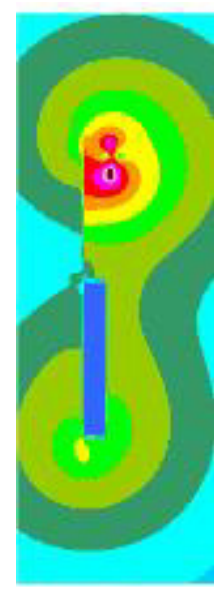

$\mathrm{d}=31 \mathrm{~mm}$

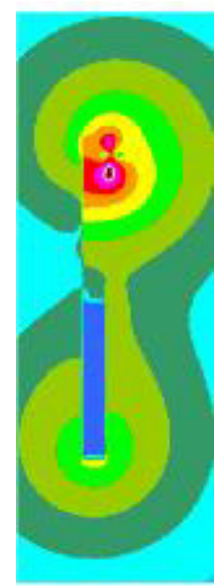

$\mathrm{d}=38 \mathrm{~mm}$
$\mathrm{dbV} / \mathrm{m}$

$\square 85-90$ $75-80$ 70-75

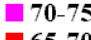
- 65-70 60-65

55-60

-50-55

45-50

घ 40-45

- 35-40

$-35-35$
-30

- 25-30 posiciones de la batería. Banda de GSM.

Los efectos de la presencia de la batería sobre la distribución de campos en DCS se ilustran en la Figura 5.41. En este caso, al ser la longitud de onda menor que en el caso de GSM, la posición de 
la batería tiene mayor influencia. Se puede observar que los campos en la parte posterior del PCB son de mayor intensidad cuando no hay batería, o cuando ésta se encuentra muy cerca del parche. En este caso el acoplamiento capacitivo entre la batería y la antena es muy elevado. Por otra parte, al desplazar la batería hacia la parte inferior del terminal, se reduce el campo en la cara del plano de masa más próxima al usuario.

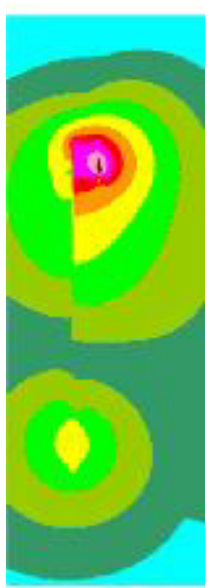

Sin batería

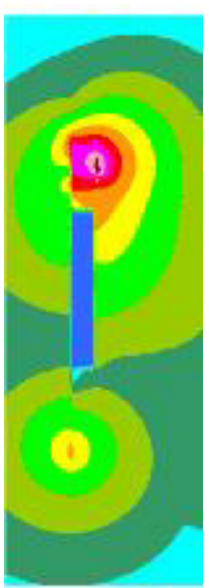

$\mathrm{d}=10 \mathrm{~mm}$

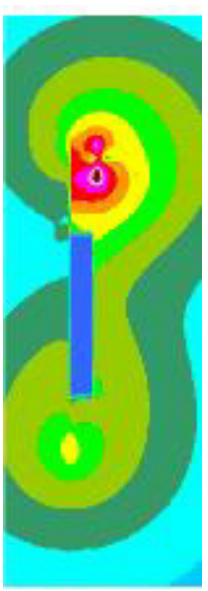

$\mathrm{d}=17 \mathrm{~mm}$

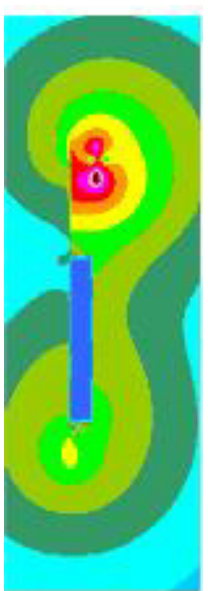

$\mathrm{d}=24 \mathrm{~mm}$

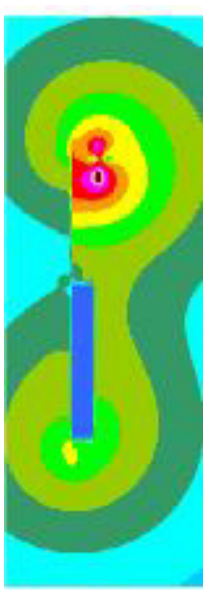

$\mathrm{d}=31 \mathrm{~mm}$

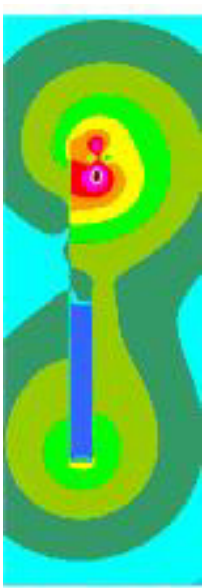

$\mathrm{d}=38 \mathrm{~mm}$
$\mathrm{dbV} / \mathrm{m}$

-75-80

$70-75$

65-70

60-65

55-60

$50-55$

$45-50$

40-45

- 35-40

30-35

25-30

20-25 posiciones de la batería. Banda de DCS.

\subsubsection{Efecto de la inclinación.}

Como se ha mencionado anteriormente, el diseño de los terminales está determinado siguiendo criterios estéticos y de marketing. Por lo tanto, es raro encontrar líneas rectas y formas regulares. Para optimizar la utilización del volumen disponible para la antena, puede resultar necesario adaptar la superficie de ésta al contorno de la tapa de plástico de la parte posterior del terminal, tal y como se ilustra en la Figura 5.42.

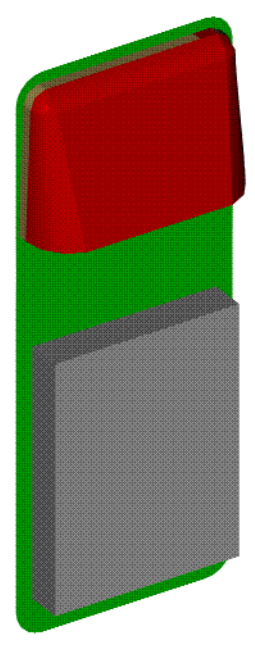

Figura 5.42: Volumen destinado a la antena (en rojo) en un terminal típico. 
En este caso se consideró una antena de doble banda contenida en un volumen de $26 \mathrm{~mm}$ x $40 \mathrm{~mm} \mathrm{x}$ $8 \mathrm{~mm}$, con una altura constante de $8 \mathrm{~mm}$ sobre la pletina [5.45]. Una variación de esta configuración consiste en inclinar la antena, disminuyendo su altura $\mathrm{h}_{1}$ en la parte superior del terminal, tal y como se muestra en la Figura 5.43. En esta zona está situado el punto de alimentación y la impedancia es menor. La altura de la antena se incrementa linealmente hasta alcanzar $\mathrm{h}_{2}=8 \mathrm{~mm}$ el extremo capacitivo, en la parte inferior de la antena.

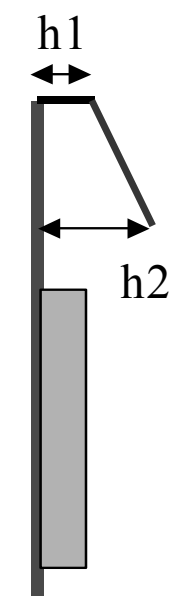

Figura 5.43: Posición de la antena respecto al PCB del terminal.

Las pérdidas de retorno simuladas para diferentes valores de $\mathrm{h}_{1}$ se ilustran en la Figura 5.44. Se puede observar que la variación de la $\mathrm{h}_{1}$, y por tanto de la inclinación de la antena, se traduce en dos efectos: un cambio en las frecuencias de resonancia, y una modificación del ancho de banda.

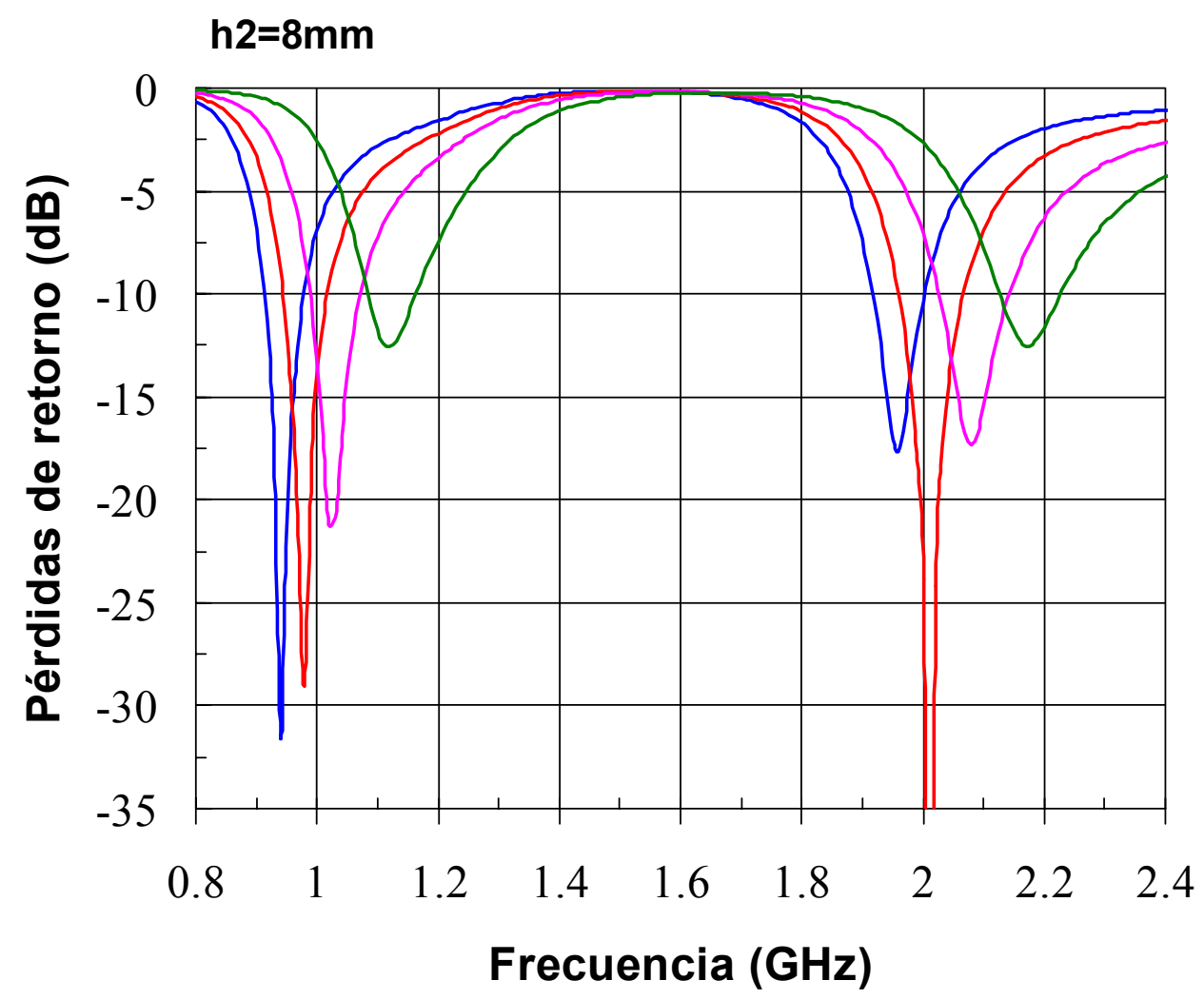

$-\mathrm{h} 1=8 \mathrm{~mm}-\mathrm{h} 1=6 \mathrm{~mm}-\mathrm{h} 1=4 \mathrm{~mm}-\mathrm{h} 1=2 \mathrm{~mm}$

Figura 5.44: Pérdidas de retorno diferentes valores de $h_{1}$. 
En la Figura 5.45 se muestra es primero de los efectos. Al aumentar el valor de $\mathrm{h}_{1}$ se incrementa también la longitud eléctrica del parche para cada uno de los dos modos excitados, así como el volumen total de la antena. Por lo tanto la frecuencia de resonancia se reduce.

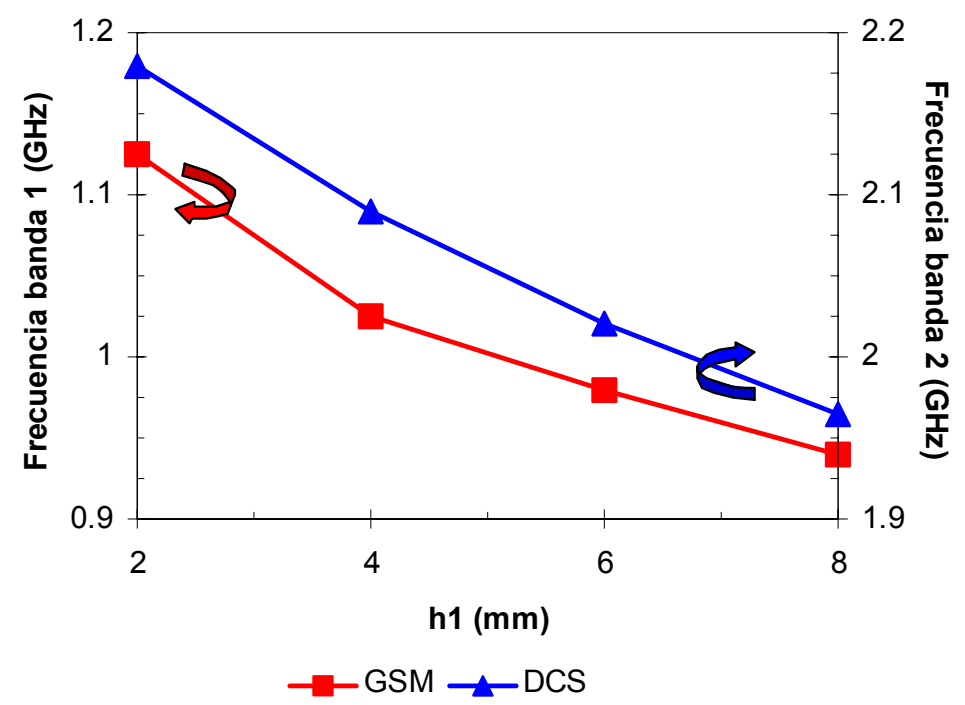

Figura 5.45: Frecuencia de resonancia en función de $h_{1}$, la altura de la antena en la parte superior del terminal $\left(\mathrm{h}_{2}=8 \mathrm{~mm}\right)$

Por otra parte, al incrementar $h_{1}$ se observa una disminución del ancho de banda. Dicha disminución se muestra en la Figura 5.46, para la cual se ha tenido en cuenta el ancho de banda relativo definido para $S_{11}<-7,5 \mathrm{~dB}$. El hecho de que el ancho de banda sea mayor para valores pequeños de $h_{1}$ puede deberse al efecto inductivo introducido al acortarse tanto el pin de alimentación como el cortocircuito. Esto compensaría el aumento de capacidad entre el parche y el plano de masa.

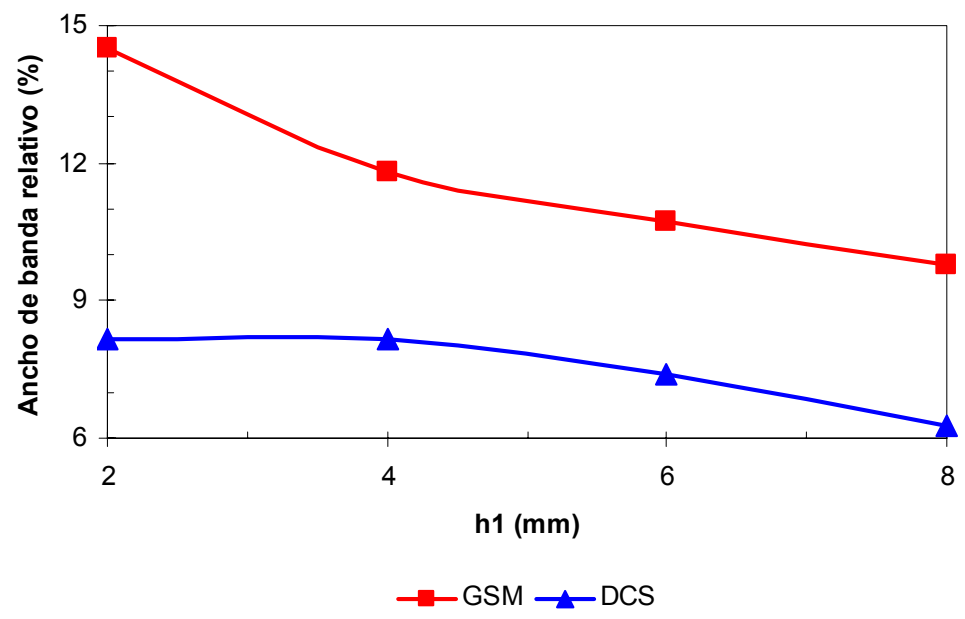

Figura 5.46: Ancho de banda relativo en función de $\mathrm{h}_{1}\left(\mathrm{~h}_{2}=8 \mathrm{~mm}\right)$.

Los efectos de la inclinación de la antena sobre la distribución del campo eléctrico para los valores extremos de $h_{1}$ en un plano perpendicular al terminal se muestran en la Figura 5.47 y la Figura 5.48 para las bandas de bandas de GSM y DCS, respectivamente. Se puede observar en ambos casos cómo, al reducir la altura de la antena en su parte superior se obtienen mayores densidades de campo eléctrico, al aumentar la capacidad entre el parche y la pletina. Paralelamente, al inclinar la 
antena acortando $h_{1}$ se reduce el valor del campo eléctrico en la parte inferior del terminal, por lo que disminuirían las pérdidas en la mano del usuario.

Además, en el caso de la banda de DCS, representada en la Figura 5.48, se observa que en el caso de la antena inclinada se reduce también el campo en la parte posterior del terminal, limitando así las posibles pérdidas en la cabeza del usuario.
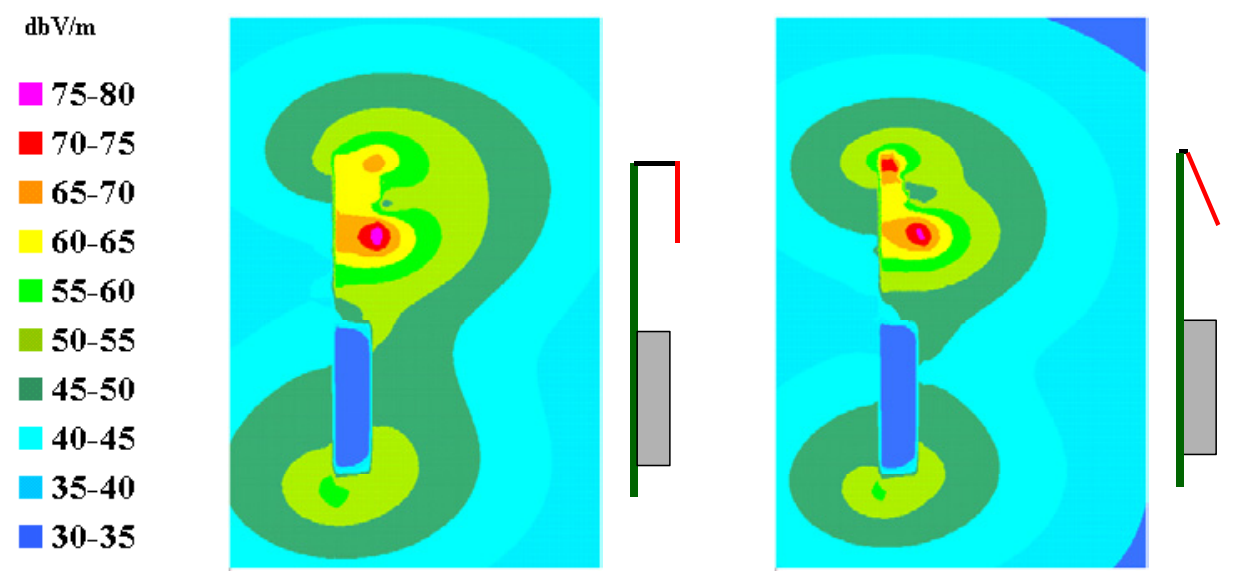

Figura 5.47: Distribución del campo eléctrico en un corte perpendicular al plano de masa para la banda de GSM. Izquierda: $\mathrm{h}_{1}=\mathrm{h}_{2}=8 \mathrm{~mm}$, derecha: $\mathrm{h}_{1}=2 \mathrm{~mm}, \mathrm{~h}_{2}=8 \mathrm{~mm}$.
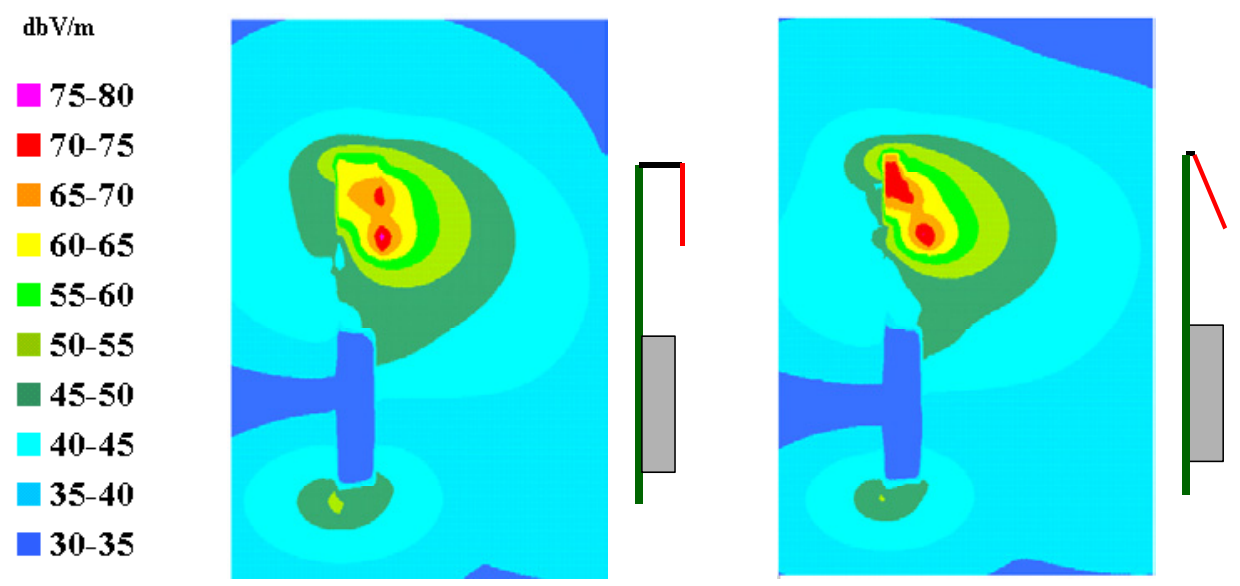

Figura 5.48: Distribución del campo eléctrico en un corte perpendicular al plano de masa para la banda de DCS. Izquierda: $\mathrm{h}_{1}=\mathrm{h}_{2}=8 \mathrm{~mm}$, derecha: $\mathrm{h}_{1}=2 \mathrm{~mm}, \mathrm{~h}_{2}=8 \mathrm{~mm}$.

\subsection{CONCLUSIÓN.}

En este capítulo se ha comprobado cómo es posible obtener una antena dual para terminales móviles mediante la inserción de un filtro espolón a lo largo del perímetro de un parche radiante. Al mismo tiempo, hemos descartado el uso de una antena impresa convencional, cuyas características no se adaptan a las exigencias de los terminales de comunicaciones móviles, en términos de tamaño y ancho de banda. También se estudiarás los efectos de diferentes elementos 
del terminal sobre las características del nuevo elemento radiante integrado.

A partir de aquí, los conceptos que se analizarán estarán más o menos basados en la PIFA dual que se ha estudiado en este capítulo, con algunas modificaciones adicionales.

\subsection{Biblografía.}

[5.1] Maci, S. y Biffi Gentilli, G., 'Dual-Frequency Patch Antennas', IEEE Antennas and Propagat. Magazine, 1997, vol. 39, nº 6, pp. 13-20.

[5.2] Wong, K.L. y Chen, W.S., 'Compact Microstrip Antenna with Dual-Frequency Operation', Electronics Lett., 1997, vol. 33, nº 8, pp. 646-647.

[5.3] Schaubert, D.H., 'Multilayer and Parasitic Configurations', en 'Handbook of Microstrip Antennas', editado por J.R. James y P.S. Hall, Peter Peregrinus Ltd, para IEE, Londres, 1989.

[5.4] Kumar, G. y Gupta, K.C., 'Broadband Microstrip Antennas using Additional Resonators GapCoupled to Radiating Edges', IEEE Trans. on Antennas and Propag., 1984, vol. 32, pp. 13751379.

[5.5] Kumar, G. y Gupta, K.C., 'Non-Radiating Edges and Four-Edges Gap-Coupled Multiple Resonators, Broadband Microstrip Antennas', IEEE Trans. on Antennas and Propag., 1985, vol. 33, n 2, pp. 173-178.

[5.6] Pues, H. et al., 'Wideband Quasi-Log-Periodic Microstrip Antenna', IEE Proceedings, Part H, 1981, vol. 128, n $^{\circ}$, pp. 159-163.

[5.7] Hall, P.S., 'New Wideband Microstrip Antenna using Log-Periodic Technique', Electronics Lett., 1980, vol. 16, n 4, pp. 127-128.

[5.8] Puente, C., Romeu, J., Pous, R., Garcia, X. y Benitez, F., 'Fractal Multiband Antenna Based on the Sierpinski Gasket', Electronics Lett., 1996, vol. 32, n 1, pp. 1-2.

[5.9] Puente-Baliarda, C., Romeu, J., Pous, R. y Cardama, A., 'On the Behavior of the Sierpinski Multiband Fractal Antenna', IEEE Trans. on Antennas and Propag., 1998, vol. 46, $\mathrm{n}^{\circ} 4$, pp. 517-524.

[5.10] Schaubert, D.H., Farrar, F.G., Sindoris, A. y Hayes, S.T., Microstrip Antennas with Frequency Agility and Polarization Diversity', IEEE Trans. on Antennas and Propagat., 1981, vol. 29, nº 1 pp. 118-123.

[5.11] Waterhouse, R.B. and Shuley, N.V.,'Dual Frequency Microstrip Rectangular Patches', Electronics Lett., 1992, vol. 28, n 7, pp. 606-607.

[5.12] Bhartia, P. y Bahl, I., 'A Frequency Agile Microstrip Antenna', IEEE Antennas and Propagat. 
Society. Symp., 1982, pp. 304-307.

[5.13] Hall, R.C., 'Full-Wave Aperture Coupled Patch Antenna', Electronics Lett., 1993, vol. 9, n 24, pp. 2073-2074.

[5.14] El Yazidi, M., Himdi, M. y Daniel, J.P., 'Aperture Coupled Microstrip Antenna for Dual Frequency Operation', Electronics Lett., 1993, vol. 29, n 17, pp. 1506-1508.

[5.15] Pozar, D.M., 'Trimming Stubs for Microstrip Feed Networks and Patch Antennas', IEEE Antennas and Propagat. Soc. Newsletter, Dec. 1987, pp. 26-28.

[5.16] Sánchez-Hernández, D. y Robertson, I.D., 'Analysis and Design of a Dual Band Circularly Polarised Microstrip Patch Antenna', IEEE Transactions on Antennas and Propagat., 1995, vol. 43, n 2, pp. 201-205.

[5.17] Bates, R. N., 'Design of Microstrip Spur-Line Band-Stop Filters', IEE Int. Journal of Microwaves, Optics and Acoustics, 1997, vol. 1, nº 6, pp. 209-214.

[5.18] Lu, J.H., Yu, H.C. y Wong, K.L., 'Compact Circular Polarisation Design for EquilateralTriangular Microstrip Antenna with Spur Lines’, Electronics Lett., 1998, vol. 34, n²1, pp. 1989-1990, Oct.

[5.19] Jones, E.M.T. y Bolljahn, J.T., 'Coupled-Strip Transmission-Lines Filters and Directional Couplers', IRE Trans. Microwave Theory and Tech., 1956,vol. 5, pp. 75-81.

[5.20] Bryant, T.G. y Weiss, J.A., 'Parameters of Microstrip Transmission Lines and of Coupled Pairs of Microstrip Lines', IEEE Trans. on Microwave Theory and Tech., 1968, vol. 16, $\mathrm{n}^{\circ}$ 12, pp. 1023-1027.

[5.21] Judd, S.V., Whiteley, I., Clowes, R.J. y Rickard, D.C., 'An Analytical Method for Calculating Microstrip Transmission Line Parameters', IEEE Trans. on Microwave Theory and Tech., 1970, vol. 18, $\mathrm{n}^{\circ} 2$, pp. 78-87.

[5.22] Getsinger, W.J, 'Microstrip Dispersión Model', IEEE Trans. on Microwave Theory and Tech., 1973, vol. 21, $\mathrm{n}^{\circ} 1$, pp. 34-39.

[5.23] De Ronde, F.C. y Shammas, S., 'MIC Bandfilters using Open Ring Resonators', 4th European Microwave Conf. Proceedings, 1974, pp. 531-535.

[5.24] Garg, R. y Bahl, I.J., 'Microstrip Discontinuities', Int. Journal of Electronics, 1978, vol. 45, $\mathrm{n}^{\circ} 1$, pp. 81-87.

[5.25] Benedek, P. y Silvester, P., 'Equivalent Capacitances for Microstrip Gaps and Steps', IEEE Trans. on Microwave Theory and Tech., 1972, vol. 20, $\mathrm{n}^{\circ}$ 11, pp. 729-733.

[5.26] Nguyen, C., Hsieh, C. y Ball, D.W., 'Millimeter Wave Printed Circuit Spurline Filters', IEEE Microwave Theory and Tech. Int. Symp. Digest, 1983, pp. 98-100.

[5.27] Bahl, I.J. y Bhartia, P., 'Microstrip Antennas', Artech House, Dedham, MA, 1980.

[5.28] 1998 GIL Technologies Product Catalogue. 
[5.29] Serrano-Vaello, Á., y Sánchez-Hernández, D., 'Printed Antennas for Dual-Band GSM/DCS1800 Mobile Handsets', Electronics Lett., 1998, vol. 34, n² 2, pp. 140-141.

[5.30] Martínez-Vázquez, M., Martínez-González, A., Ávila-Navarro, E. y Sánchez-Hernández, D., 'Dual-Band Dual-Mode Printed Bow-Tie Antennas Integrated in Handheld Terminals for Wireless Communications', $28^{\text {th }}$ European Microwave Conf. Proceedings, 1998, pp. 16.

[5.31] Martínez-González, A., Sánchez-Aguilar, M.A., Martínez-Vázquez M. y SánchezHernández, D., 'A Comparison of Dual-Band Spur-Line Printed Antennas for Hand-Held Handsets', Microwave and Optical Technology Lett., 2001, vol. 30, n 3, pp. 205-207.

[5.32] Taga, T., 'Analysis of Planar Inverted-F Antennas and Antenna Design for Portable Radio Equipment', en 'Analysis, Design, and Measurement of Small and Low Profile Antennas', editado por K. Hirasawa y M. Haneishi, Artech House, Norwood, MA, 1992.

[5.33] Pedersen, G.F. y Bach Andersen, J., 'Integrated Antennas for Hand-Held Telephones with Low Absorption', IEEE Vehicular Technology Conf., 1994, pp. 1537-1540.

[5.34] McLean, S., 'A Re-Examination of the Fundamental Limits on the Radiation Q of Electrically Small Antennas', IEEE Trans. on Antennas and Propagat., 1996, vol. 44, n 5 , pp. 672-676.

[5.35] Staub, O., Zürcher, J.F., Skrivervik, A. y Mosig, J.R., 'Miniaturizing Antennas for Personal Communications Systems', $30^{\text {th }}$ European Microwave Conf. Proceedings, 2000, p. 348353.

[5.36] Luebbers, R., Chen, L., Uno, T. y Adachi, S., 'FDTD Calculation of Radiation Patterns, Impedance, and Gain for a Monopole Antenna on a Conducting Box', IEEE Trans. on Antennas and Propagat., 1992, vol. 40, n 12, pp. 1577-1583.

[5.37] Sánchez Hernández D. y Martínez Vázquez, M., 'Antena dual para terminales móviles', ${ }^{\circ}$ de solicitud: P-200002704, Oficina Española de patentes y Marcas.

[5.38] Martínez-Vázquez, M., Geissler, M., Heberling D. y Sánchez-Hernández, D., 'Compact Dual-Band Antenna for Mobile Handsets', Microwave and Optical Tech. Lett., 2002, vol. $32, \mathrm{n}^{\circ} 2$, pp. $87-88$.

[5.39] Geissler, M. Heberling, D. y Wolff, I., 'Properties of Integrated Handset Antennas', Millenium Conf. on Antennas and Propag. AP-2000, 2000.

[5.40] Vainikainen, P., Ollikainen, J., Kivekäs, O. y Kelander, I., 'Performance Analysis of Small Antennas Mounted on Mobile Handsets', COST 259 Final Workshop - Mobile and Human Body Interaction, Bergen, 2000.

[5.41] Manteuffel, D., Bahr, A. y Wolff, I., 'Investigation on Integrated Antennas for GSM Mobile Phones', Millenium Conf. on Antennas and Propag. AP-2000, 2000.

[5.42] Manteuffel, D., Bahr, A., Heberling, D. y Wolff, I., 'Design Considerations for Integrated 
Mobile Phone Antennas', IEE Int. Conf. on Antennas and Propag., 2001, vol. 1, pp. 252255 .

[5.43] Wu, T.Y. y Wong, K.L., 'On the Impedance Bandwidth of a Planar Inverted-F Antenna for Mobile Handsets, Microwave and Optical Technology Lett., 2002, vol. 32, n 4, pp. 249251.

[5.44] Kivekäs, O., Ollikainen, J., Lehtiniemi, T. y Vainikainen, P., 'Effect of the Chassis Length on the Bandwidth, SAR, and Efficiency of Internal Mobile Phone Antennas, Microwave and Optical Technology Lett., 2003, vol. 36, $\mathrm{n}^{\circ}$ 6, pp. 457-462.

[5.45] Arkko, A., 'Effect of Ground Plane Size on the Free-Space Performance of a Mobile handsel PIFA Antenna', IEE Int. Conf. on Antennas and Propag., 2003, vol. 1, pp. 316319.

[5.46] Martínez-Vázquez, M., Geissler, M. y Heberling, D., 'Volume Considerations in the Design of Dual-Band Handset Antennas', IEEE Antennas and Propagat. Society. Symp., 2001. 


\section{Capítulo 6: ANTENAS INTEGRADAS MULTIBANDA.}

Haríamos muchas más cosas si creyéramos que son muchas menos las imposibles.

Malesherbes

\subsection{INTRODUCCIÓN.}

En la actualidad, el campo de la integración de elementos radiantes multibanda en el interior de terminales móviles constituye uno de los más activos tanto en lo referente a antenas como a telefonía móvil..

En el capítulo anterior se describía cómo era posible implementar una antena integrada en un terminal de comunicaciones móviles dual mediante la combinación de un parche radiante con un filtro espolón. En este capítulo se pretende profundizar en este sentido, a partir de los resultados obtenidos, y analizando nuevas geometrías, que incluyen la utilización de parches acoplados y parásitos o la introducción de ranuras en la estructura. Esta estrategia coincide con la utilizada habitualmente para lograr una operación multibanda sin aumentar el tamaño total de la antena, doblando el parche o insertando en él ranuras, abiertas o cerradas [6.1]-[6.3].

Algunas de las soluciones presentadas aquí han sido concebidas para plataformas específicas, por lo que habrá que modificar la geometría del parche para que pueda ser integrado en el terminal. Por otra parte, se tiende a extender el modo de operación a un número mayor de estándares. Por lo tanto se presentan también resultados referentes a antenas de tres o más bandas.

\subsection{ANTENAS DUALES PLANAS.}

\subsubsection{Antena triangular con filtro espolón.}

Tal y como se ha mencionado anteriormente, es necesario adaptar la forma del parche radiante al diseño del terminal móvil. En este sentido resulta particularmente interesante la antena presentada 
en la Figura 6.1. Consiste en un parche triangular truncado de $40 \mathrm{~mm}$ de base y $26 \mathrm{~mm}$ de altura, cortocircuitado en su parte superior. A lo largo del perímetro se inserta un filtro espolón para lograr una segunda frecuencia de resonancia. El parche tiene una altura de $10 \mathrm{~mm}$ sobre el plano de masa [6.1].

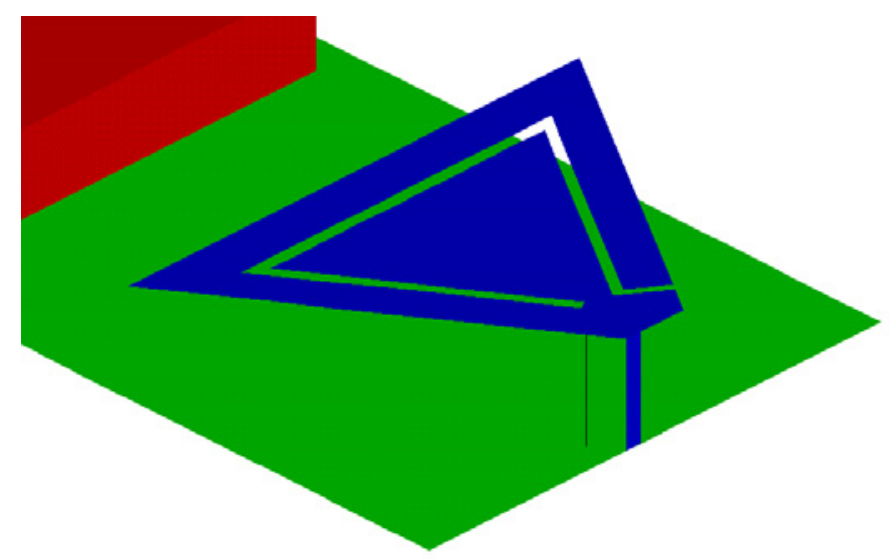

Figura 6.1: Estructura de la PIFA triangular con filtro espolón.

La implementación de la antena se muestra en la fotografía de la Figura 6.2.

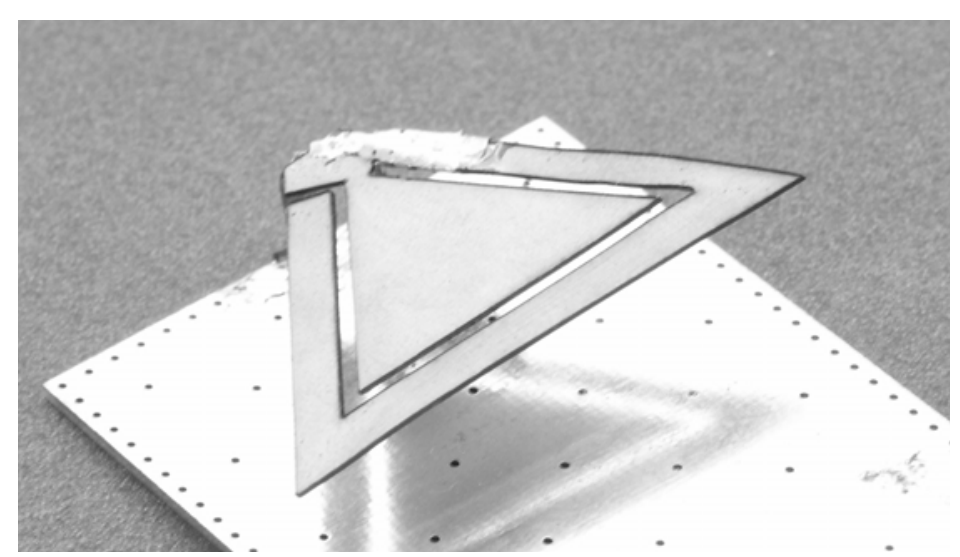

Figura 6.2: PIFA triangular con filtro espolón.

Los resultados de las medidas de las pérdidas de retorno de la PIFA triangular con filtro espolón se recogen en la Figura 6.3. En ellos se aprecia claramente las dos frecuencias de resonancia de la antena. Se puede observar cómo el ancho de banda para GSM, que corresponde al modo introducido por el filtro espolón, es menor que en el caso de DCS, modo asociado al parche radiante.

La distribución de campos eléctrico y magnético a frecuencias de GSM se representa en la Figura 6.4. Al igual que en el caso de la PIFA del capítulo anterior, es el filtro espolón el que genera la resonancia para esta banda de frecuencia. La rama exterior del parche se comporta como un elemento resonante de longitud aproximadamente igual a un cuarto de la longitud de onda de trabajo. 


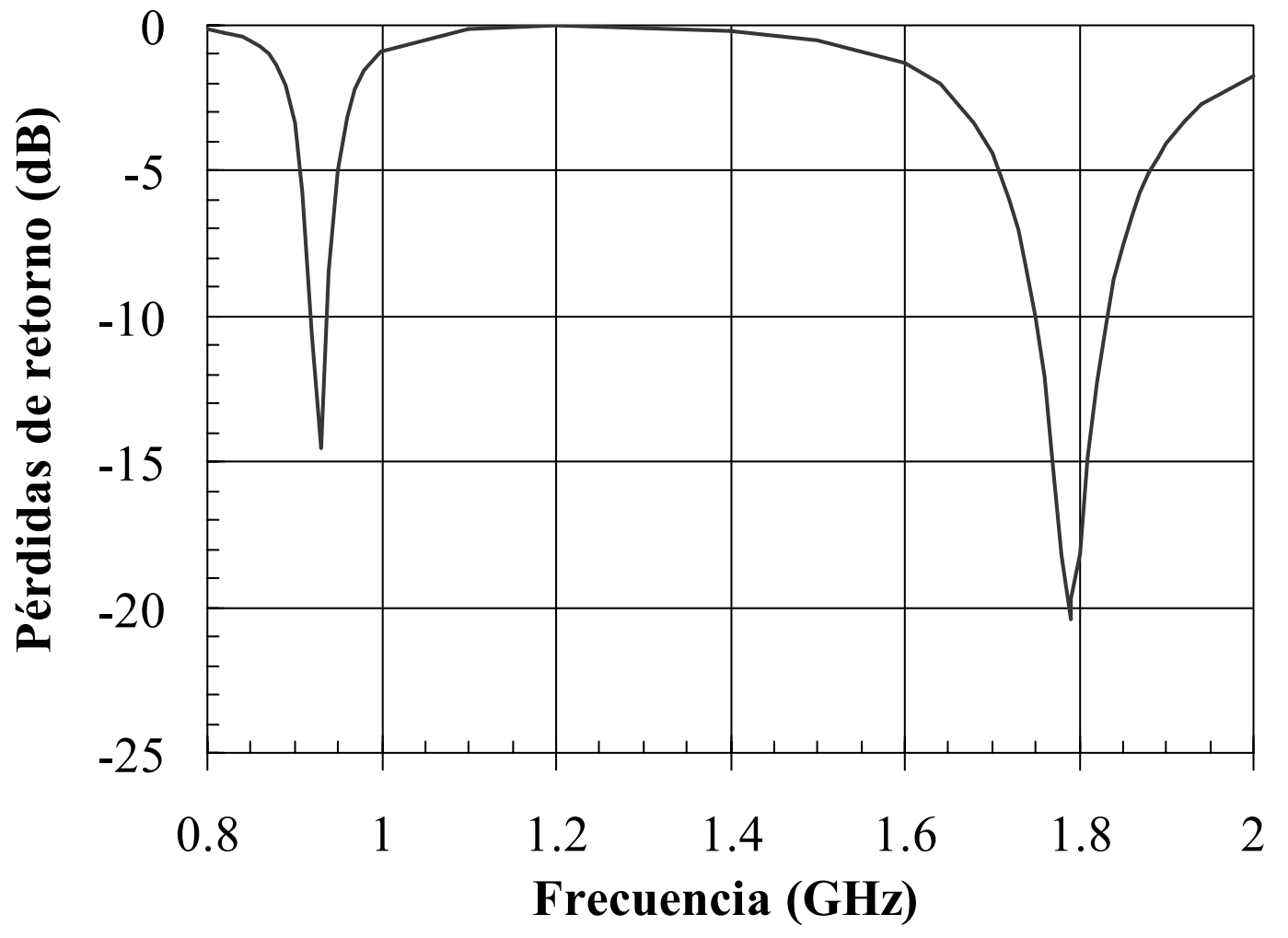

Figura 6.3: Pérdidas de retorno de la PIFA triangular con filtro espolón.

En el caso de las frecuencias de DCS, la zona activa se concentra de nuevo en la parte interior del parche, tal y como se aprecia en las distribuciones de campos de la Figura 6.5.
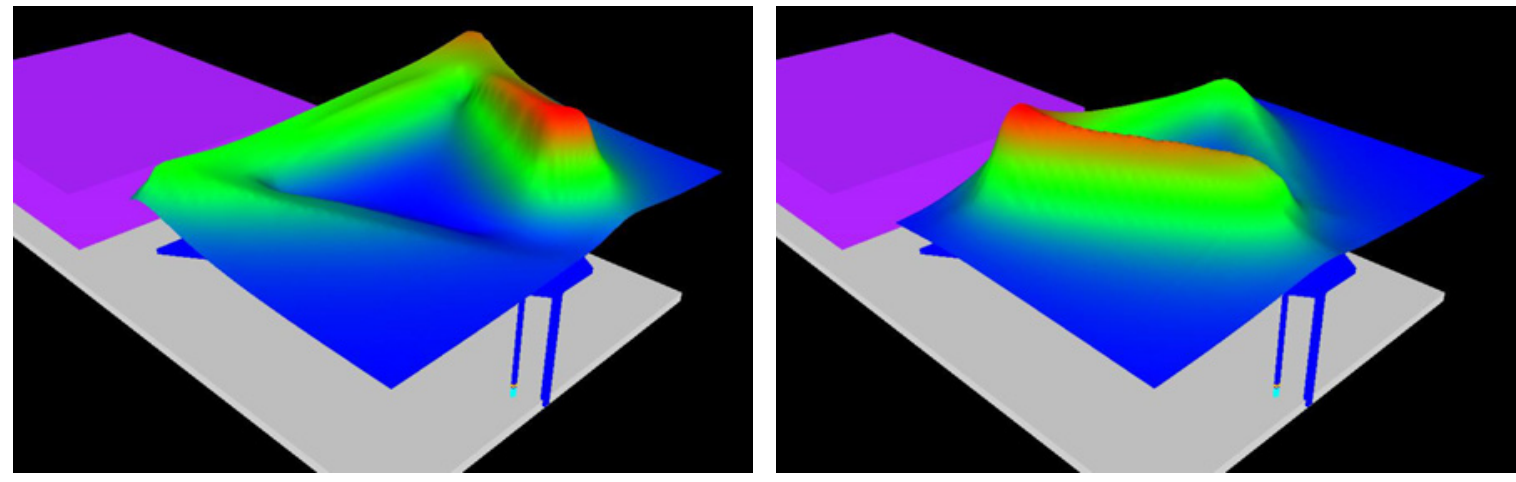

Figura 6.4: Distribución de campo eléctrico (izquierda) y magnético (derecha) de la antena triangular en la banda de GSM.
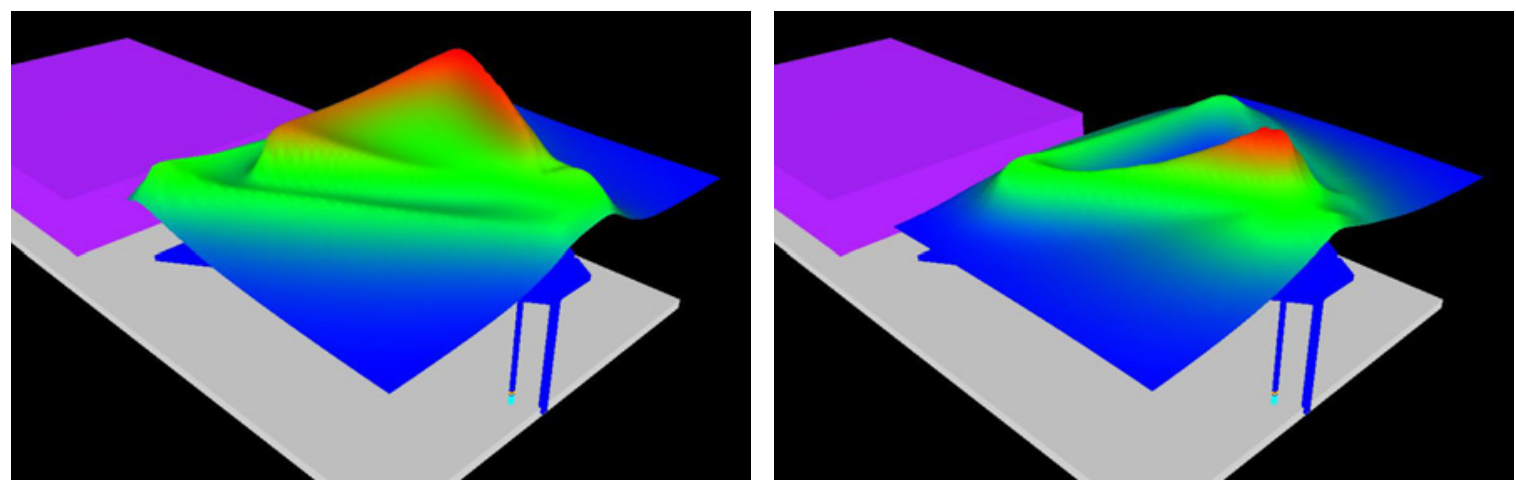

Figura 6.5: Distribución de campo eléctrico (izquierda) y magnético (derecha) de la antena triangular en la banda de DCS. 
También en este caso los diagramas de radiación resultantes, representados en la Figura 6.6, son prácticamente omnidireccionales en el plano horizontal, y con un comportamiento similar al del dipolo en $\lambda / 2$.
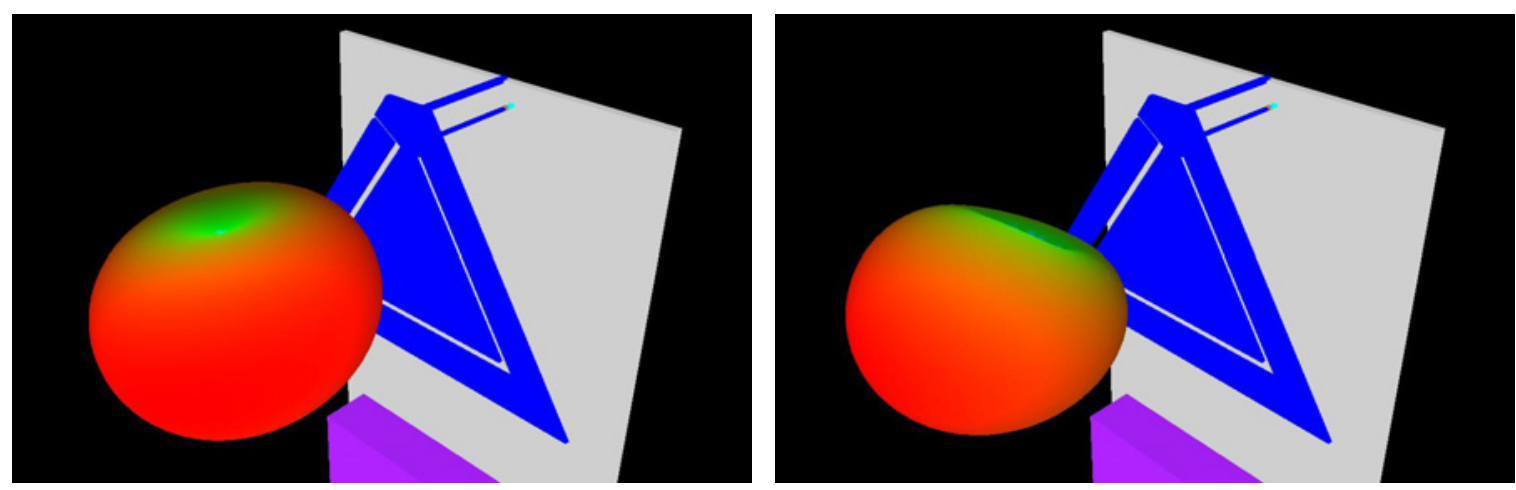

Figura 6.6: Diagrama de radiación de la antena triangular en las bandas de GSM (izquierda) y DCS (derecha).

\subsubsection{Antena $M$.}

Para mejorar las prestaciones de la antena con filtro espolón presentada en el capítulo anterior, se modificó la geometría del parche hasta llegar a la forma que se muestra en la Figura 6.7 [6.5]. Para ello se agrandó el ancho del filtro hasta el punto en que el principio de funcionamiento de la antena se ve modificado. Ahora se puede considerar que el parche consta de dos elementos radiantes acoplados por sus extremos, excitándose así un modo para cada banda de frecuencias. El tamaño total de la estructura es $39 \mathrm{~mm} \times 26 \mathrm{~mm} \times 8 \mathrm{~mm}$.

La estructura considerada incluye un soporte de plástico que garantiza la estabilidad mecánica de la misma. El efecto de este soporte dieléctrico se estudiará con más detenimiento en el capítulo siguiente.

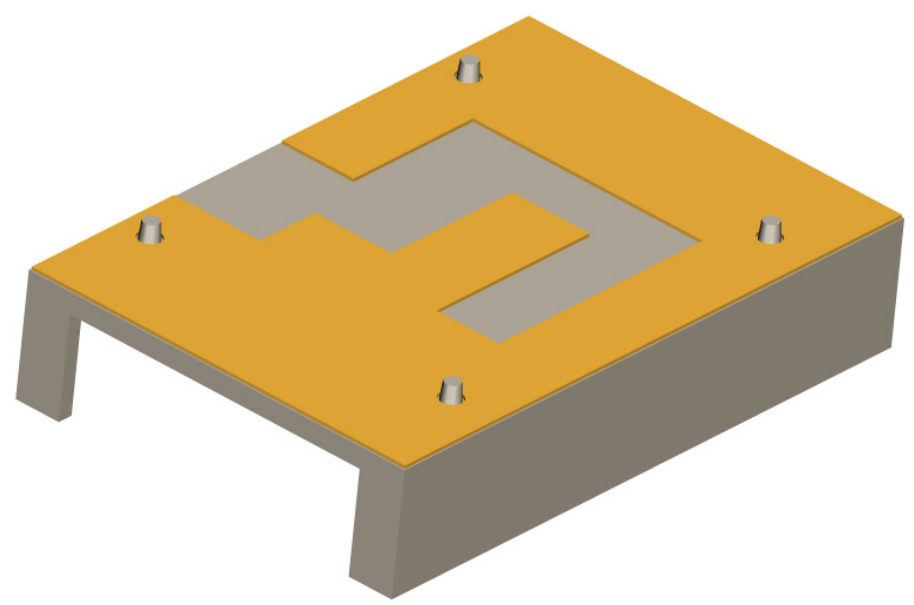

Figura 6.7: Geometría de la antena M.

Las pérdidas de retorno medidas para las bandas de GSM y DCS se pueden observar en la Figura 
6.8. Se aprecia que el ancho de banda para GSM es claramente mayor que en el caso de la antena con filtro espolón presentada en el capítulo anterior. Los valores de las pérdidas de retorno se recogen en la Tabla 6.1 .

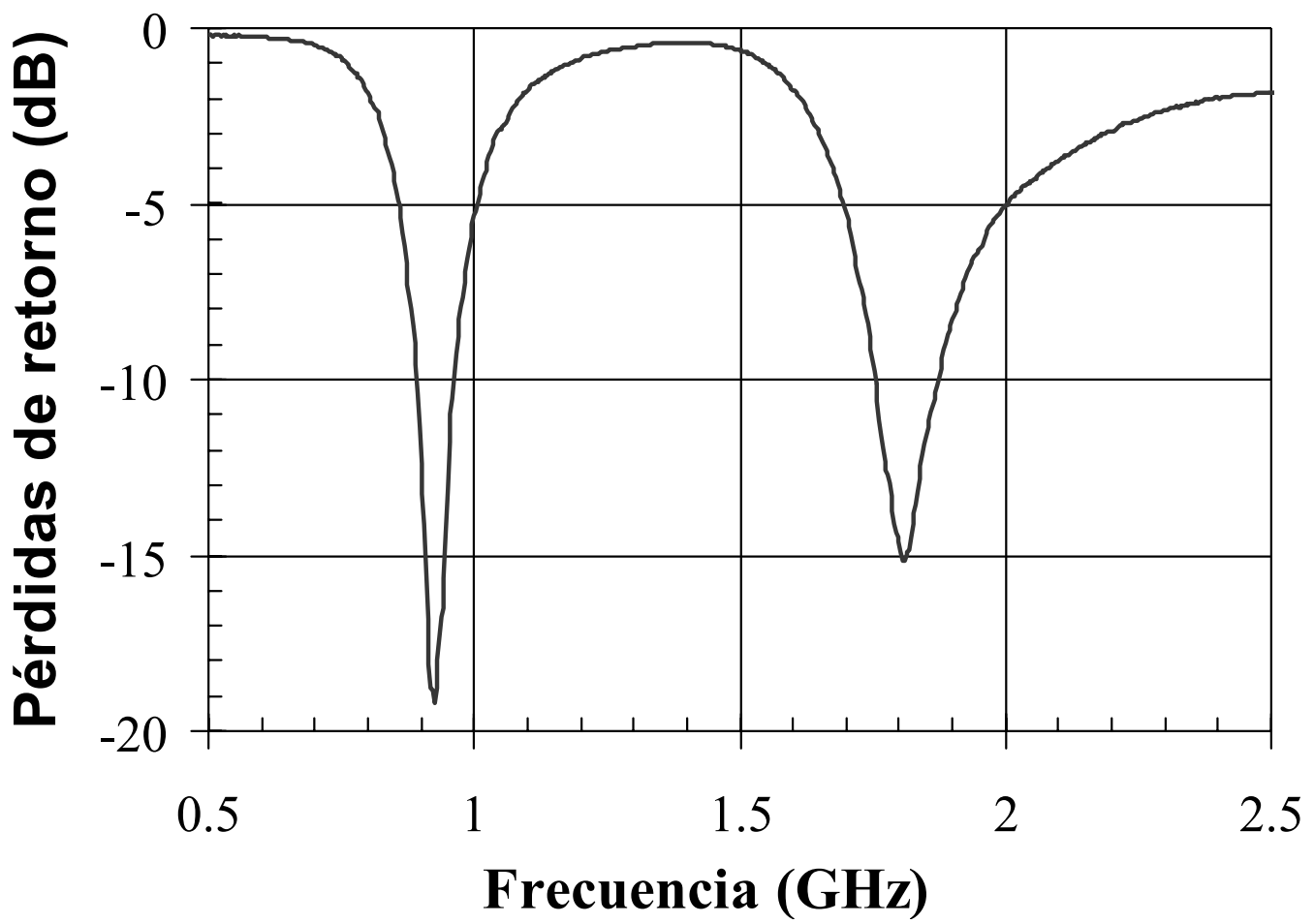

Figura 6.8: Pérdidas de retorno medidas de la antena $\mathrm{M}$.

Tabla 6.1: Características de adaptación de la antena M.

\begin{tabular}{|c|c|c|}
\hline Estándar & $\begin{array}{c}\text { Frecuencia central de } \\
\text { resonancia }\end{array}$ & $\begin{array}{c}\text { Ancho de banda } \\
\left.\mathbf{( S}_{\mathbf{1 1}}<-\mathbf{7 , 5} \mathbf{~ d B}\right)\end{array}$ \\
\hline $\mathrm{GSM}$ & $909 \mathrm{MHz}$ & $93 \mathrm{MHz}$ \\
$\mathrm{DCS}$ & $1796 \mathrm{MHz}$ & $161 \mathrm{MHz}$ \\
\hline
\end{tabular}

En la banda de GSM el mecanismo de radiación depende principalmente de la rama del parche que se extiende a lo largo de 3/4 partes del perímetro de la antena, tal y como se aprecia en la Figura 6.9. Debido al acoplamiento capacitivo entre las dos partes del parche, la longitud de este elemento es ligeramente inferior a $\lambda / 4$.
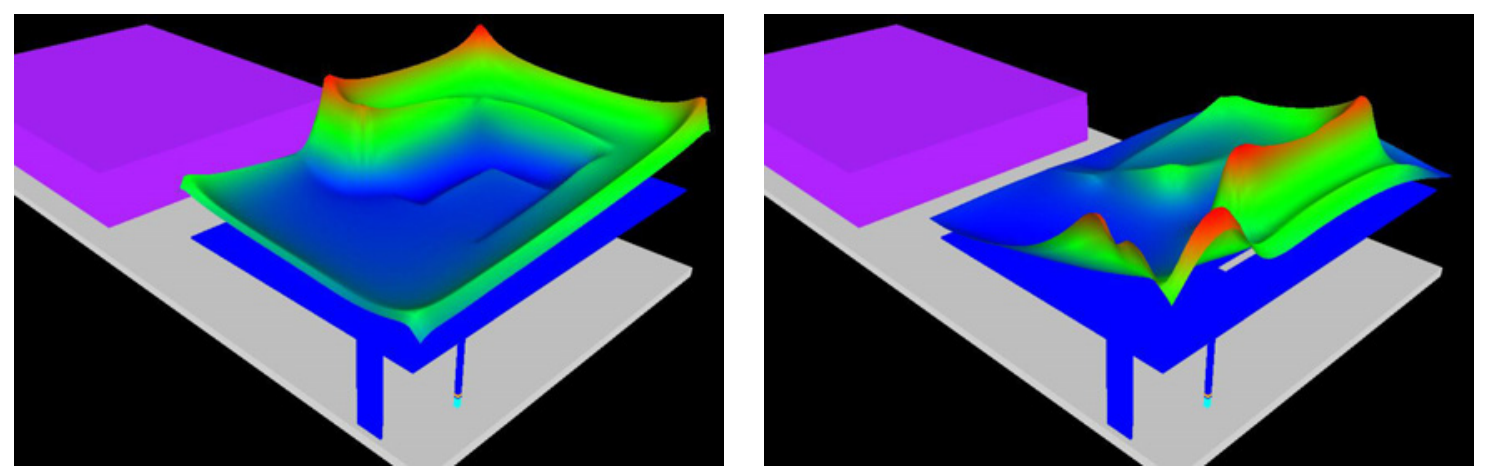

Figura 6.9: Distribución de campo eléctrico (izquierda) y magnético (derecha) de la antena M en la banda de GSM. 
En DCS, en cambio, la zona activa del parche se encuentra en el interior. En la Figura 6.10 se observa cómo aparecen máximos de campo eléctrico en las zonas en las que se produce el acoplamiento capacitivo entre ambas ramas del parche.
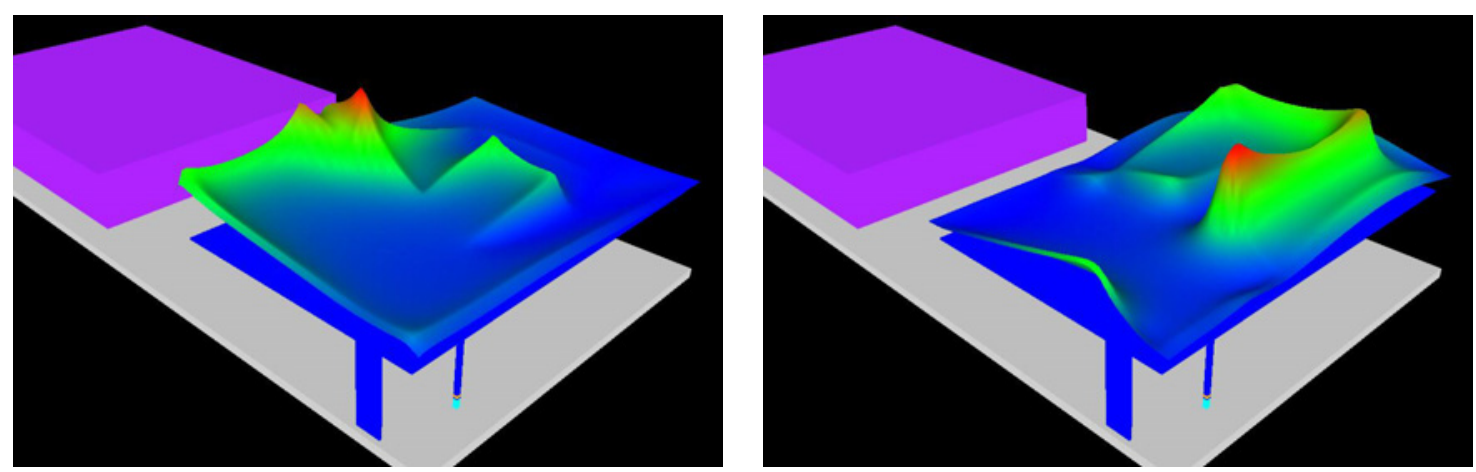

Figura 6.10: Distribución de campo eléctrico (izquierda) y magnético (derecha) de la antena M en la banda de DCS.

Estas distribuciones de campo próximo dan lugar a los diagramas de radiación de las Figura 6.11. En ellos se observa que, para las frecuencias de GSM, el comportamiento sigue siendo similar al del dipolo, mientras que en DCS la forma del diagrama de variación varía, con un máximo en la dirección de la parte trasera del terminal, donde se encuentra la antena.
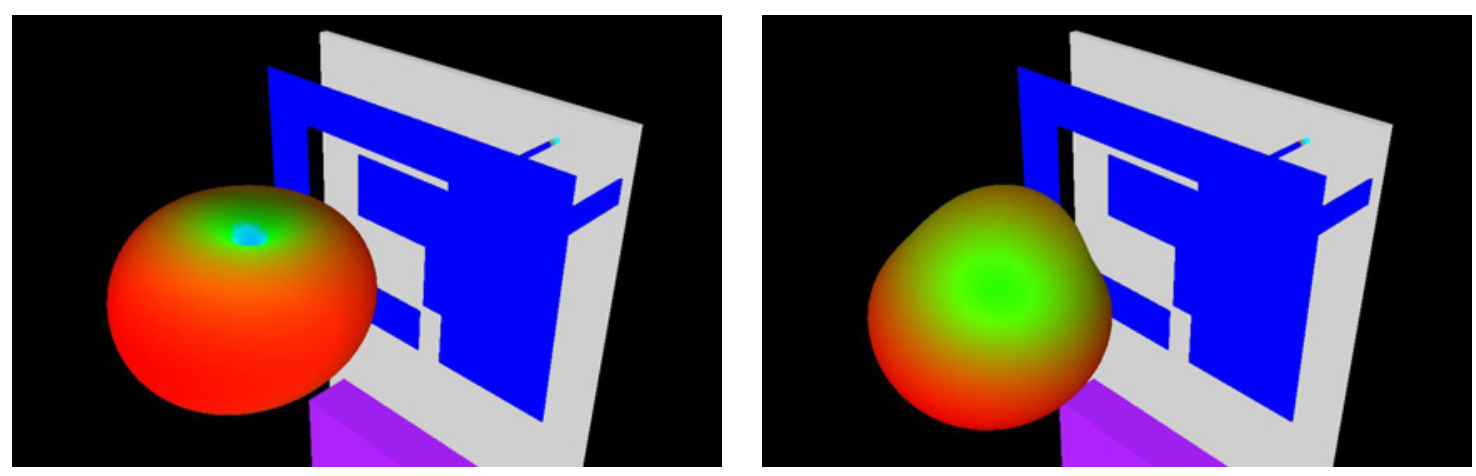

Figura 6.11: Diagrama de radiación de la antena M en las bandas de GSM (izquierda) y DCS (derecha).

En la Tabla 6.2 se representan los diagramas de radiación medidos de la antena para las frecuencias centrales de las bandas de GSM y DCS en los planos de corte principales. El sistema de coordenadas utilizado se muestra en la Figura 6.12.

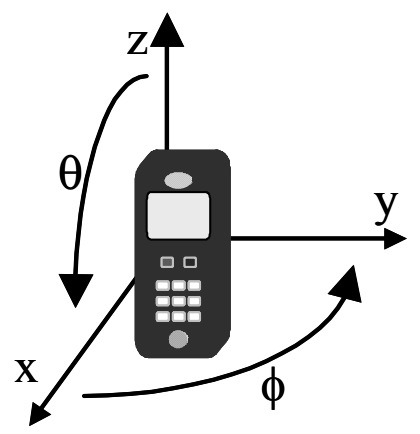

Figura 6.12: Sistema de coordenadas utilizado en las medidas de los diagramas de radiación. 


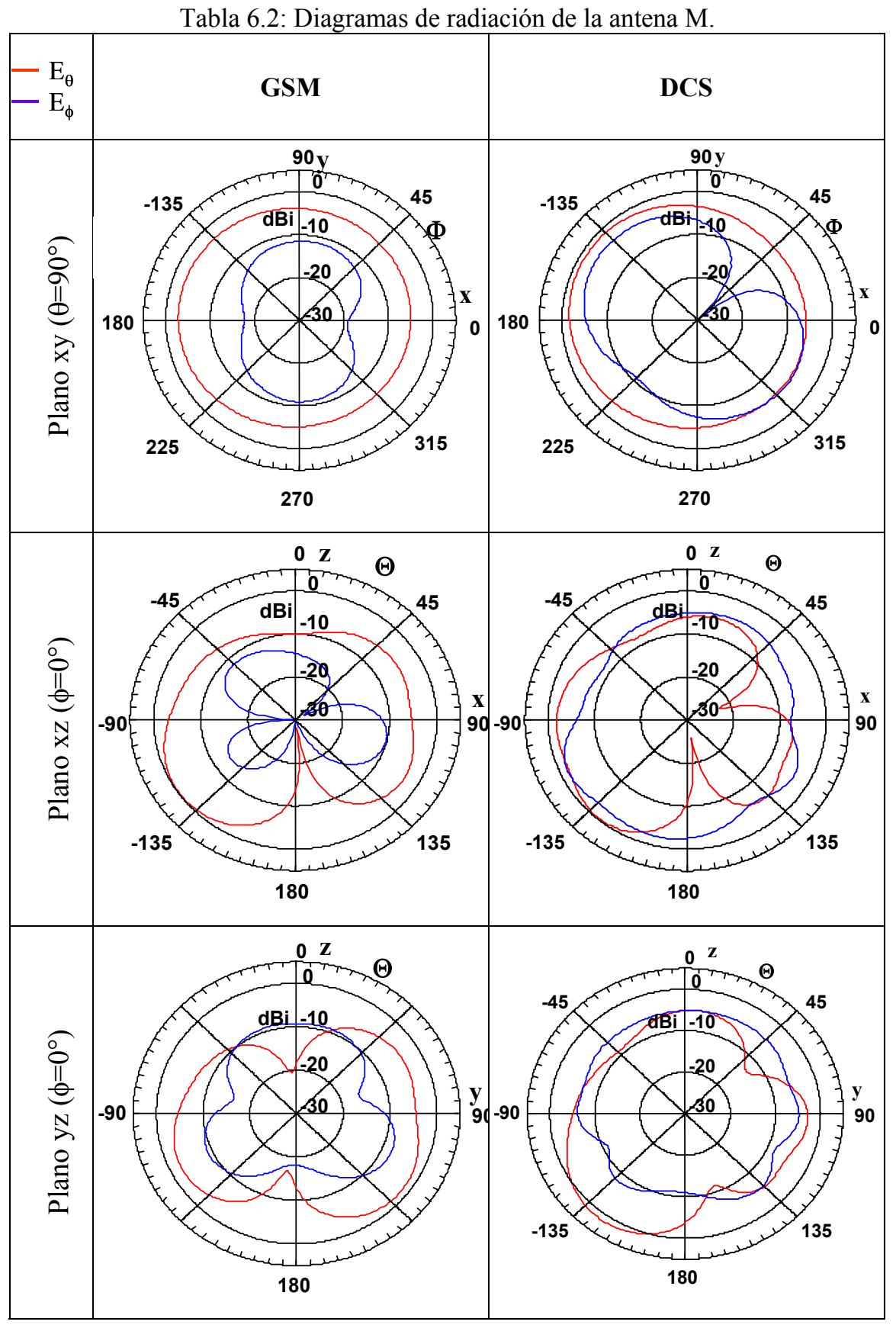

La ganancia de la antena es de 0,2 dBi para la frecuencia central de la banda de GSM, y de 1,6 dBi en la de DCS. Las formas de los diagramas coinciden con las típicas de antenas parche sobre planos de masa de dimensiones finitas utilizados en comunicaciones móviles. Se puede ver cómo los niveles de polarización cruzada son relativamente elevados. Esto no es en absoluto preocupante, dada la orientación aleatoria del terminal en condiciones normales de utilización.

\subsubsection{Antena M con conector integrado.}

Una variante de la antena anterior consiste en una combinación antena-conector para antena externa. Así, se podría conmutar automáticamente entre la antena interna y la externa, por ejemplo, 
al colocar el terminal en el dispositivo "manos libres" del coche. La estructura de la antena se muestra en la Figura 6.13.

De nuevo, para poder fabricar esta antena, es preciso estamparla sobre un soporte plástico que lo proporcione robustez mecánica. Con ello se modifica la permitividad efectiva bajo el parche. En el próximo capítulo se estudiará más en detalle este efecto.

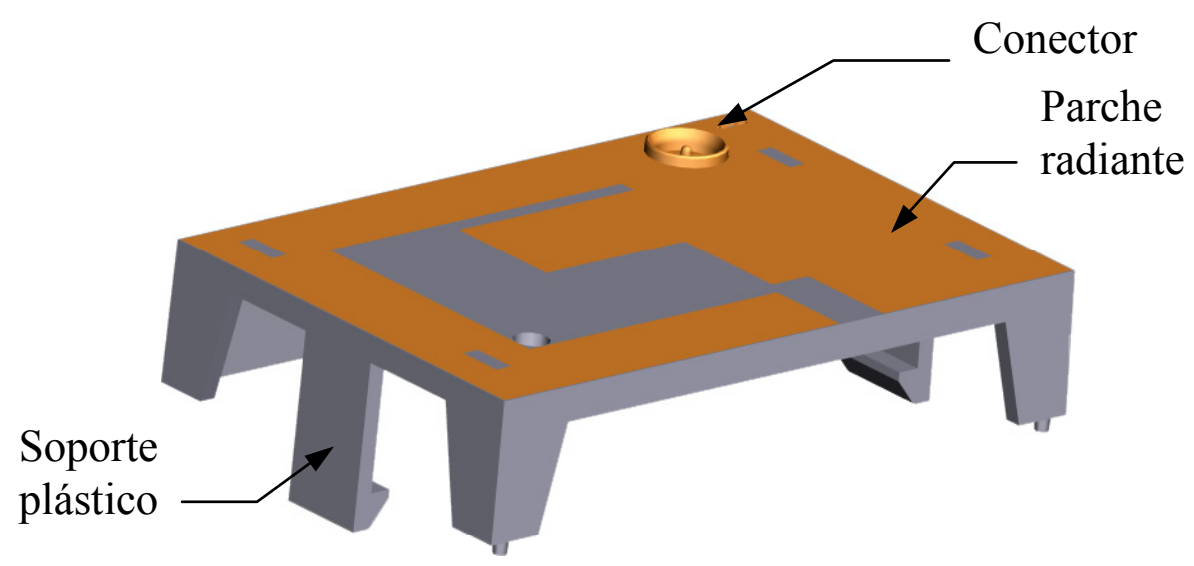

Figura 6.13: Estructura de la antena M con conector integrado.

El uso del conector supone un desplazamiento del punto de alimentación, cuyo efecto hay que compensar modificando ligeramente la forma de la antena. También hay que tener en cuenta que aumentarán en aproximadamente 0,5 a $1 \mathrm{~dB}$ las pérdidas debido a la transición entre el conector y el parche.

En la fotografía de la Figura 6.14 se muestra la antena montada en el PCB. La sujeción a este último se hace por presión, por lo que no es necesario soldadura en los puntos de contacto.

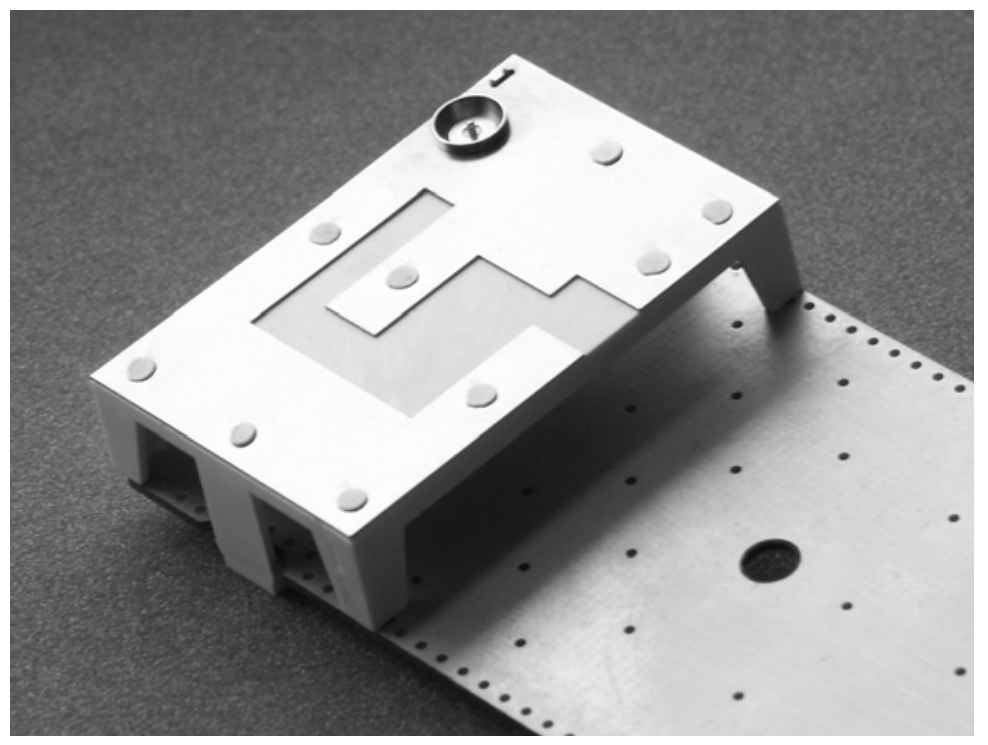

Figura 6.14: Antena $\mathrm{M}$ con conector integrado.

Las pérdidas de retorno medidas se muestran en la Figura 6.15, mientras que las características de adaptación de la antena aquí descrita se resumen en la Tabla 6.3. Para ambas bandas de frecuencia, el ancho de banda alcanzado es superior al establecido por los estándares. La desviación en 
frecuencia respecto a las frecuencia centrales de las bandas puede ser compensada a posteriori mediante pequeños retoques en la geometría del parche.

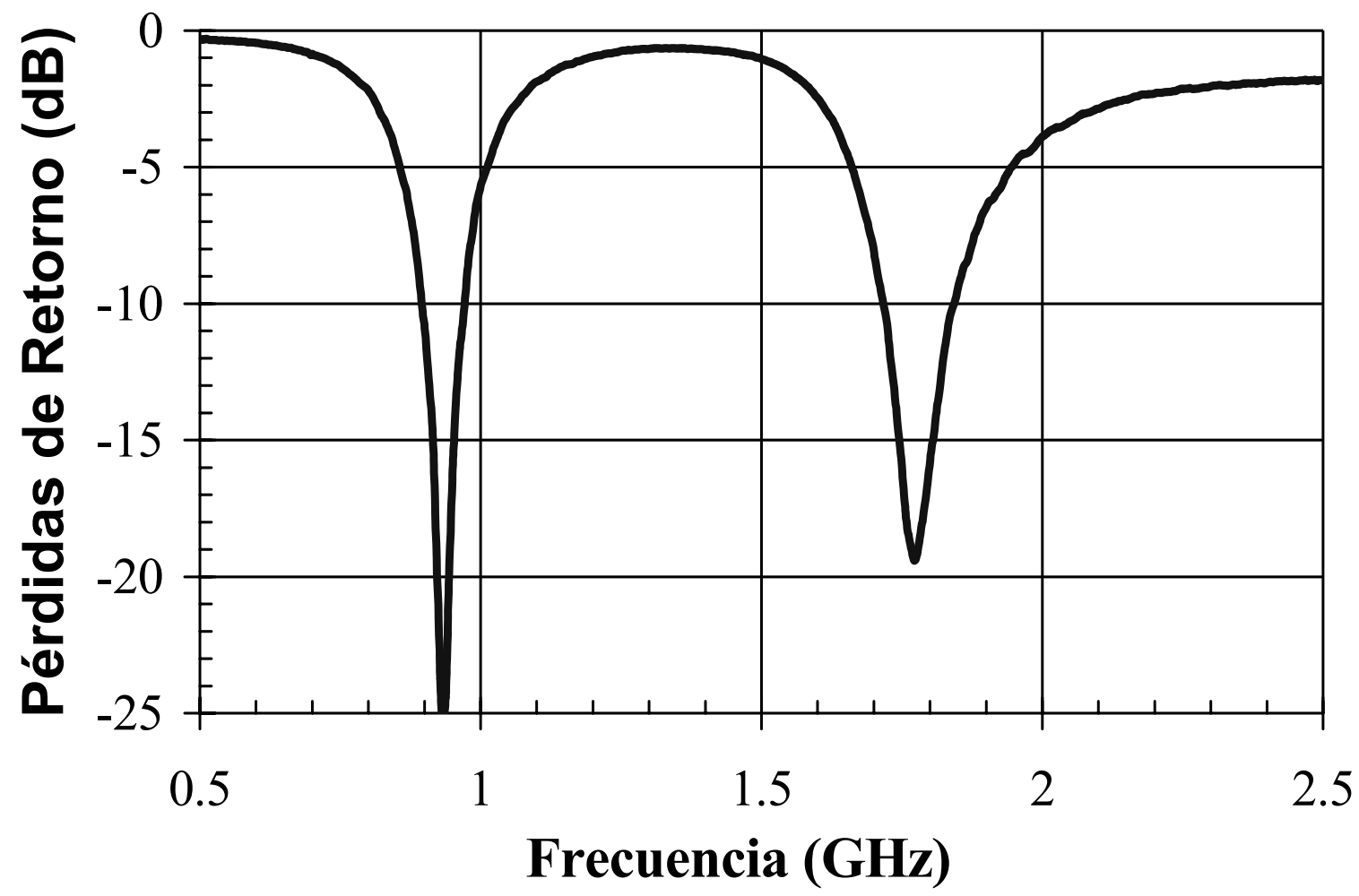

Figura 6.15: Pérdidas de retorno medidas, con y sin usuario, de la antena $\mathrm{M}$ con conector integrado.

Tabla 6.3: Características de adaptación de la antena M con conector integrado.

\begin{tabular}{|c|c|c|}
\hline Estándar & $\begin{array}{c}\text { Frecuencia central de } \\
\text { resonancia }\end{array}$ & $\begin{array}{c}\text { Ancho de banda } \\
\text { (S11<-7,5 dB) }\end{array}$ \\
\hline $\mathrm{GSM}$ & $932 \mathrm{MHz}$ & $104 \mathrm{MHz}$ \\
$\mathrm{DCS}$ & $1787 \mathrm{MHz}$ & $185 \mathrm{MHz}$ \\
\hline
\end{tabular}

En la Tabla 6.4 se incluyen los diagramas de radiación medidos de la antena en las bandas de GSM y DCS para los planos de corte principales. El sistema de coordenadas utilizado es el representado en la Figura 6.12. La antena presenta una ganancia de $-1,6 \mathrm{dBi}$ para la frecuencia central de la banda de GSM, y de - $0,7 \mathrm{dBi}$ en la de DCS. La forma de los diagramas apenas difiere ligeramente de la de la antena $\mathrm{M}$ sin conector.

\subsubsection{Antena "ring-patch".}

La antena considerada está formada por dos parches radiantes de diferentes longitudes, acoplados capacitivamente por sus extremos [6.5]. Ambos parches comparten un mismo punto de alimentación y el pin de masa. La superficie ocupada por la estructura es $37 \mathrm{~mm}$ x $21 \mathrm{~mm}$ x $8 \mathrm{~mm}$, mientras que el plano de masa mide $37 \mathrm{~mm}$ x $100 \mathrm{~mm}$. Su estructura se muestra en la Figura $6.16 \mathrm{y}$ en la fotografía de la Figura 6.17. 
Tabla 6.4: Diagramas de radiación de la antena $\mathrm{M}$ con conector integrado.

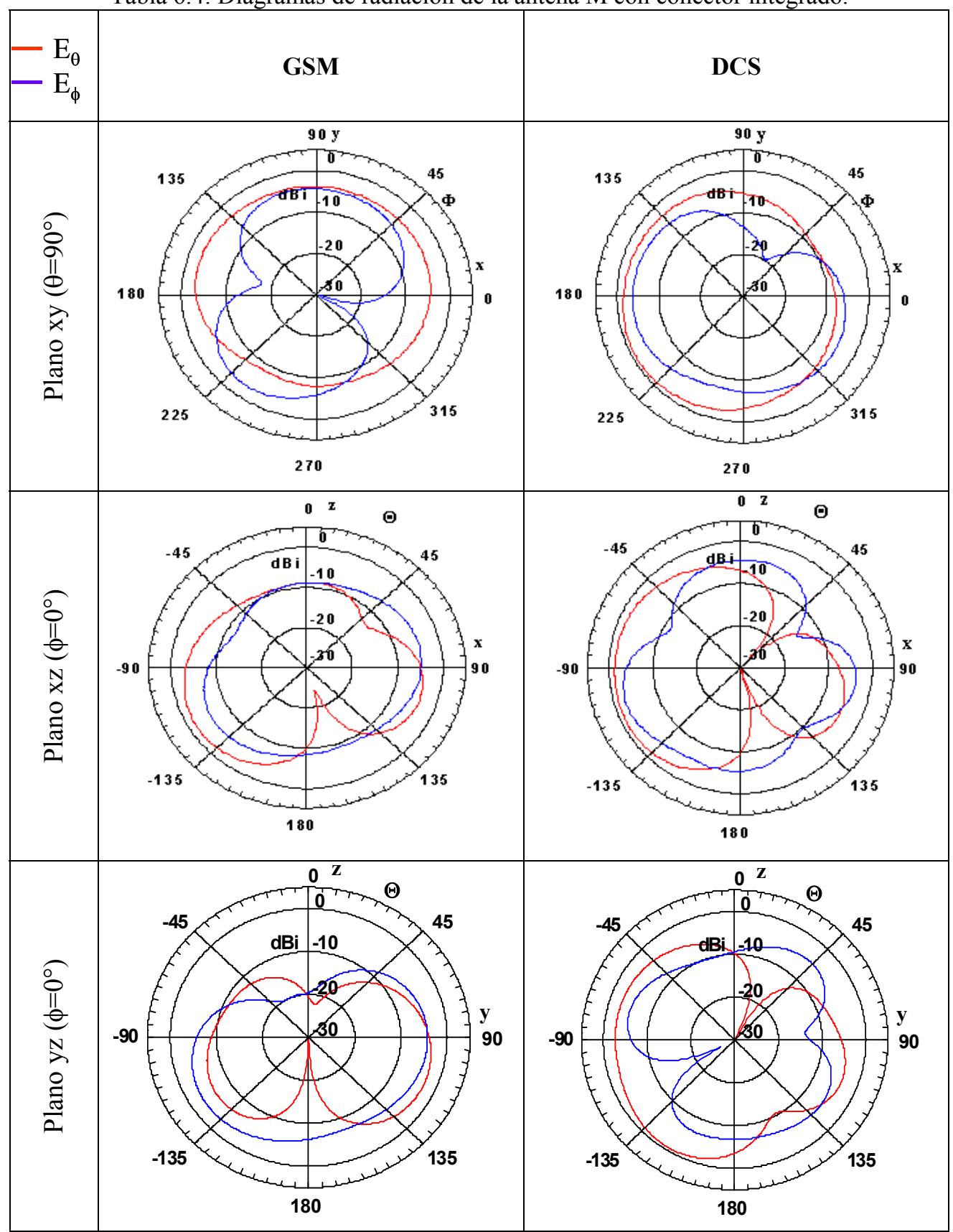

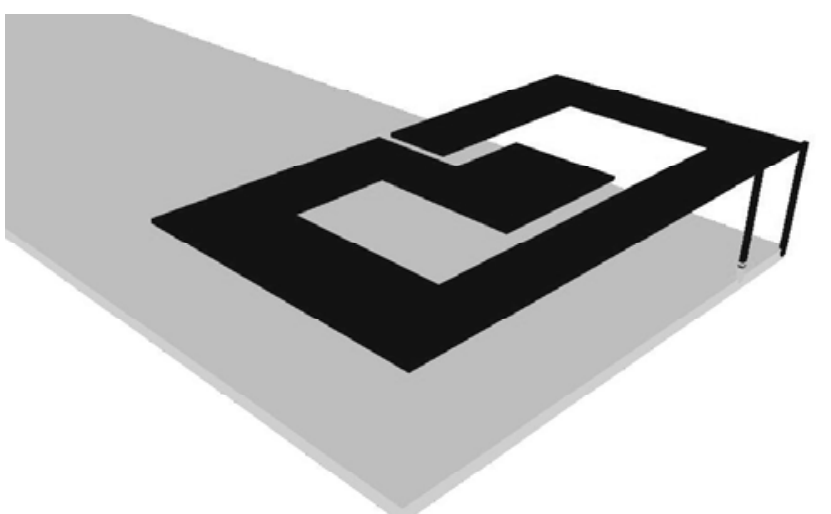

Figura 6.16: Estructura de la antena "ring-patch". 


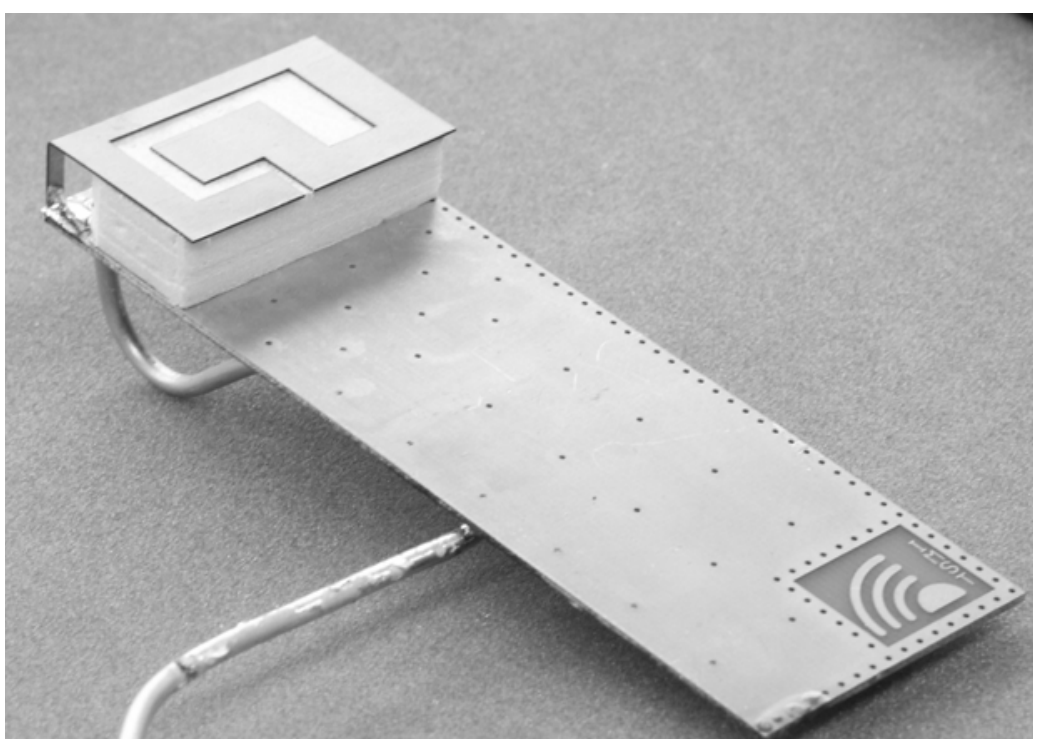

Figura 6.17: Antena "ring-patch".

En la Figura 6.18 se muestran las pérdidas retornos medidas, mientras que la Tabla 6.5 se detallan sus principales características. En este caso, debido al reducido volumen ocupado por la antena, se ha tomado el límite de $\mathrm{S}_{11}<-6 \mathrm{~dB}$ para definir el ancho de banda.

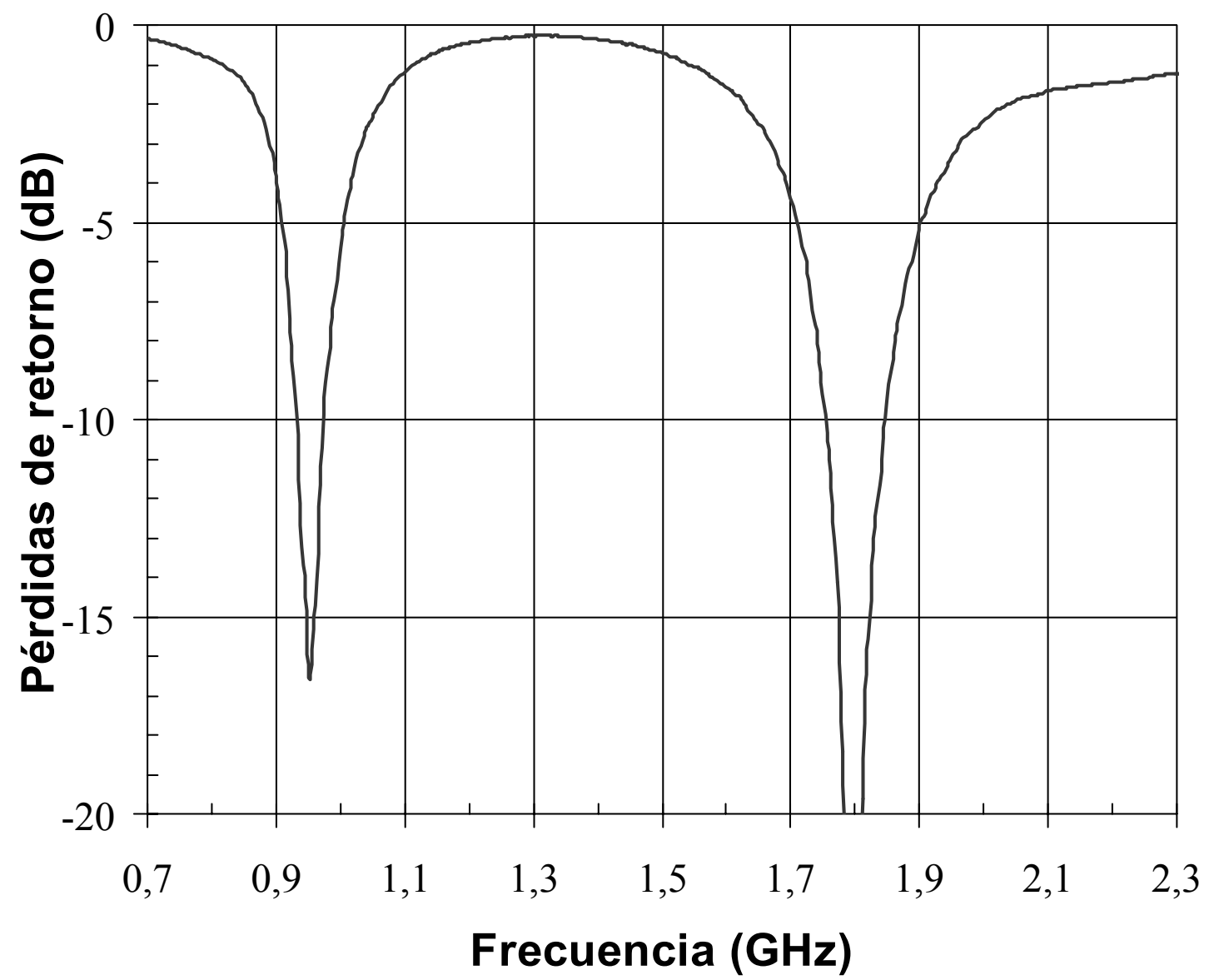

Figura 6.18: Pérdidas de retorno de la antena "ring-patch".

En la gráfica se pueden apreciar claramente las dos resonancias que aparecen en el parche, y que permiten cubrir las bandas de GSM y DCS, aunque el ancho de este último aún no es suficiente para cumplir con las normas. 
Tabla 6.5: Características de adaptación de la antena "ring-patch".

\begin{tabular}{|c|c|c|}
\hline Estándar & $\begin{array}{c}\text { Frecuencia central de } \\
\text { resonancia }\end{array}$ & $\begin{array}{c}\text { Ancho de banda } \\
\text { (S11<-6 dB) }\end{array}$ \\
\hline GSM & $953 \mathrm{MHz}$ & $89 \mathrm{MHz}$ \\
DCS & $1797 \mathrm{MHz}$ & $160 \mathrm{MHz}$ \\
\hline
\end{tabular}

En la Figura 6.19 y la Figura 6.20 se recogen las distribuciones de campo eléctrico y magnético en las bandas de GSM y DCS, respectivamente. Se aprecia claramente cómo, en cada caso, es una u otra rama del parche la que se activa, dando lugar a un modo resonante distinto.

El acoplamiento de entre los extremos permite reducir ligeramente la longitud total del parche, sin perder prestaciones en cuanto a ancho de banda y características de radiación.
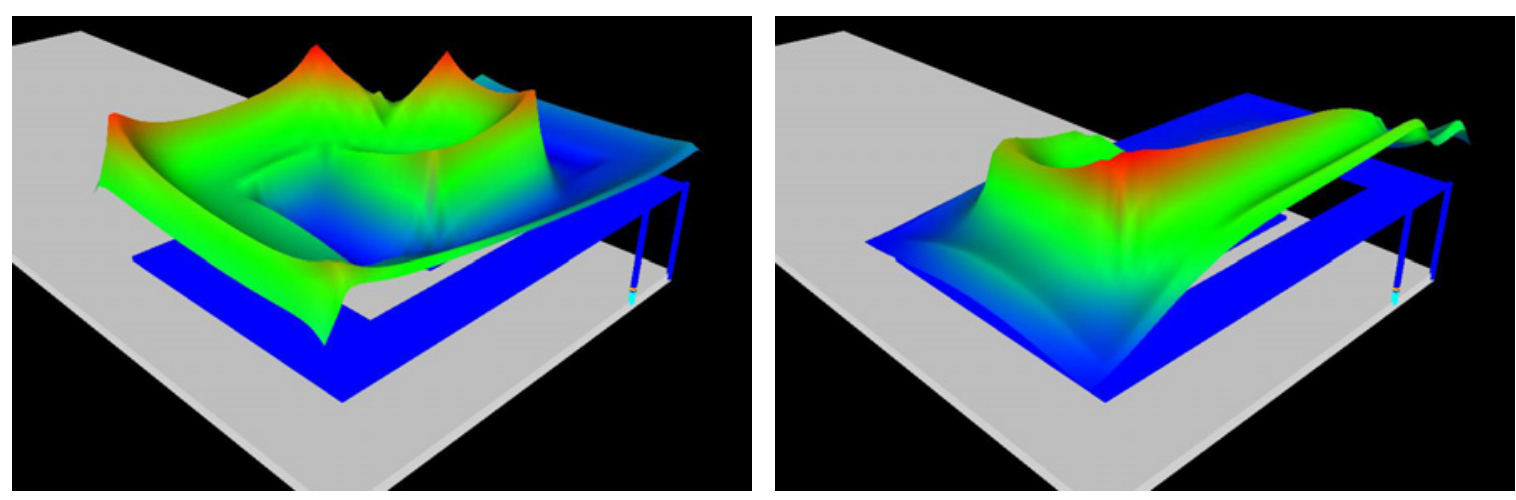

Figura 6.19: Distribución de campo eléctrico (izquierda) y magnético (derecha) de la antena "ring-patch" en la banda de GSM.
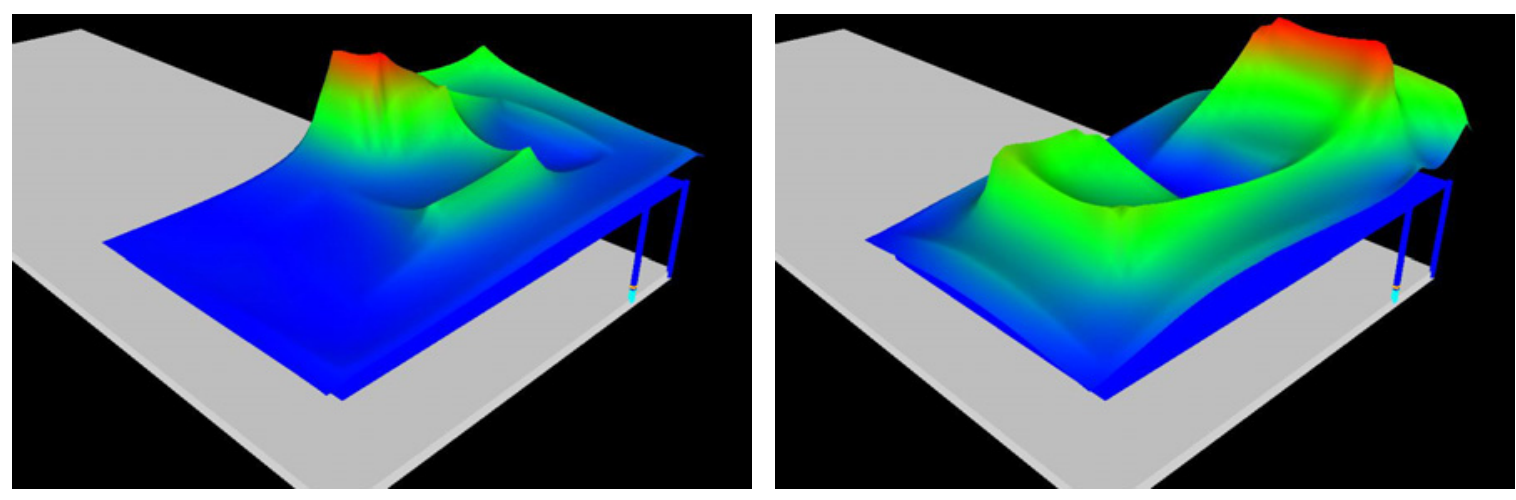

Figura 6.20: Distribución de campo eléctrico (izquierda) y magnético (derecha) de la antena "ring-patch" en la banda de DCS.

Los diagramas de radiación de la antena se muestran el la Figura 6.21. Para GSM, el comportamiento es muy similar al del dipolo, mientras que en DCS se aprecia un nulo de radiación desviado respecto de los ejes de coordenadas. 

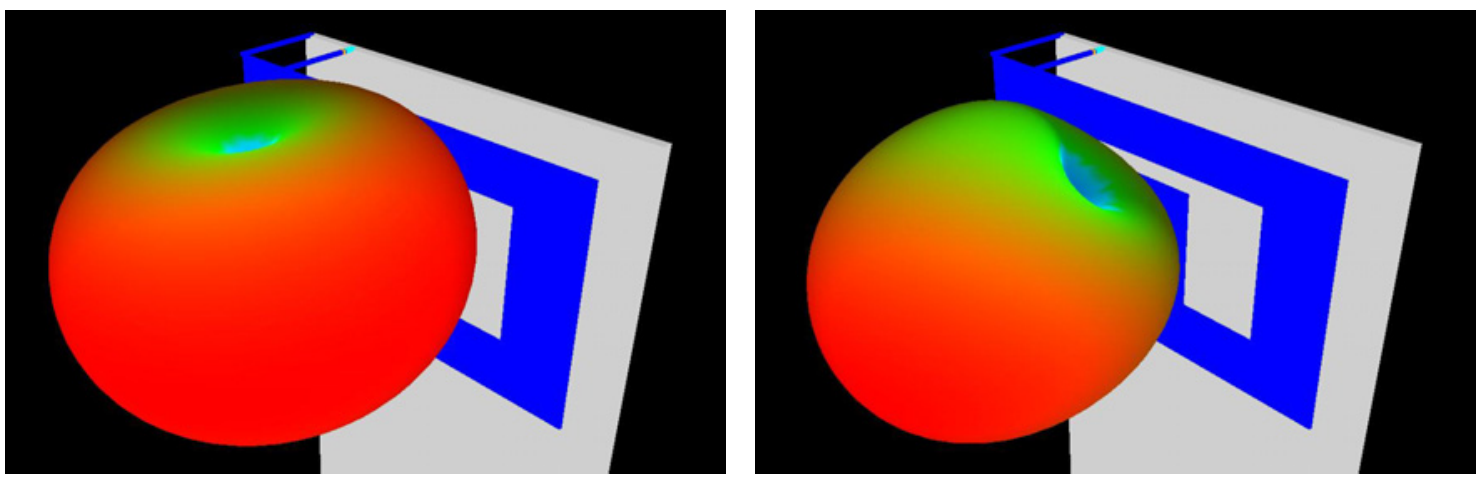

Figura 6.21: Diagrama de radiación de la antena "ring-patch" en las bandas de GSM (izquierda) y DCS (derecha).

\subsection{ANTENAS ADAPTADAS A LA FORMA DEL TERMINAL.}

Los parches presentados en los apartados anteriores ocupaban todos una superficie plana rectangular. En la mayoría de los casos reales las condiciones son distintas, puesto que hay que ajustar la forma de la antena al diseño del terminal. En este apartado se presentan ejemplos de antenas cuya forma ha sido alterada para cumplir con los requisitos geométricos del diseño.

\subsubsection{Antena "ring-patch" inclinada.}

Como ya se ha mencionado anteriormente, la forma de una antena integrada debe adaptarse a la forma exterior del terminal. Así, ésta puede tenerse en cuenta en la fase de simulación, como en el caso del modelo de la Figura 6.22. La antena considerada es una variación de la presentada en el apartado 6.2.4.

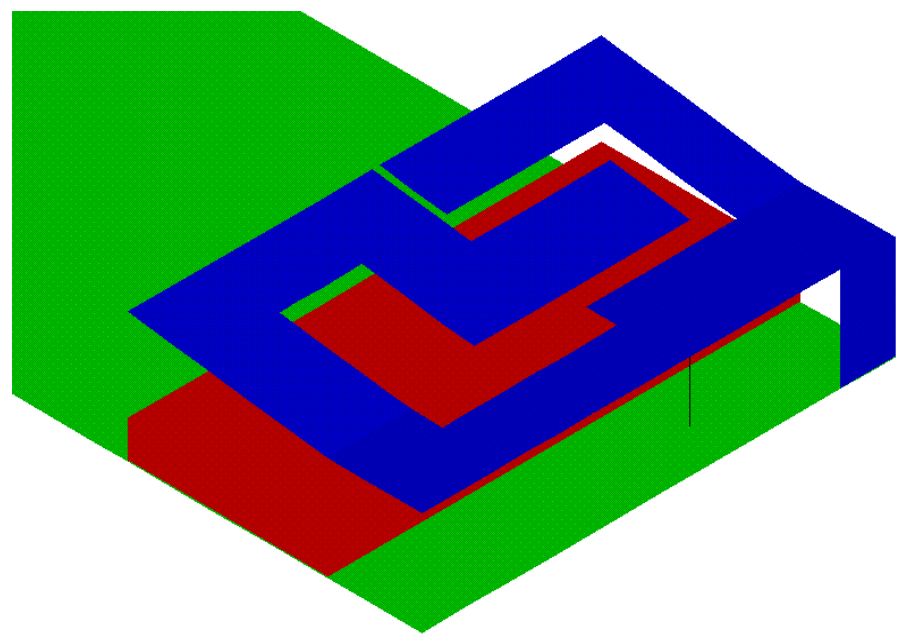

Figura 6.22: Estructura de la antena "ring-patch" adaptada al terminal. 
La superficie ocupada por la estructura es $34 \mathrm{~mm} \times 21 \mathrm{~mm}$, con una altura que varía entre $7,6 \mathrm{~mm}$ en su parte superior, donde se encuentra el punto de alimentación, y 9,5 mm en su parte más elevada. Además, hay que tener en cuenta la presencia bajo el parche de unos circuitos apantallados de $2 \mathrm{~mm}$ de espesor, con lo cual altura efectiva es aun menor.

En la foto de la Figura 6.23 se puede observar la estructura de la antena sobre un plano de masa de $34 \mathrm{~mm} \times 100 \mathrm{~mm}$. Para dar estabilidad a la estructura se colocó un bloque de Styropor bajo el parche, que no alteraba de forma significativa la permitividad efectiva, de modo que se puede considerar que ésta sigue siendo igual a la del aire.

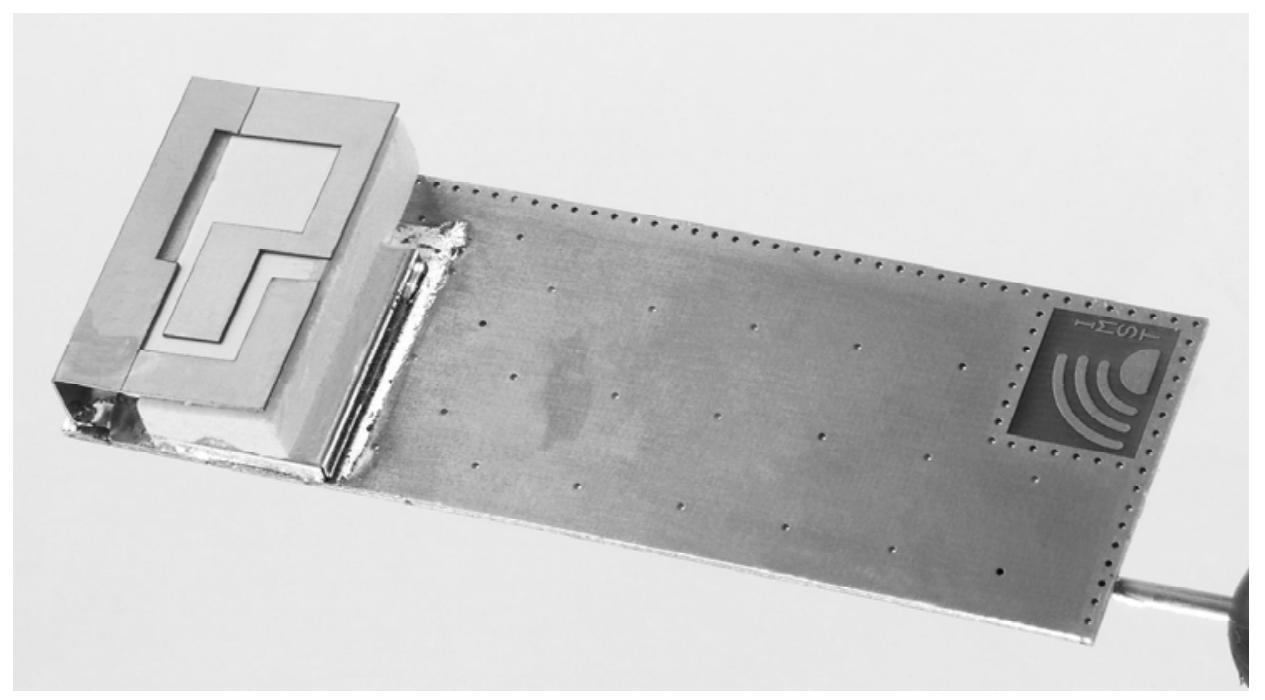

Figura 6.23: Maqueta del terminal con la antena integrada.

En la Figura 6.24 se recogen las pérdidas de retorno medidas correspondientes a esta antena. Se puede apreciar cómo, aunque se alcanza una mejor adaptación en GSM, el ancho de banda es mayor para DCS. Esto es debido en gran medida al efecto del plano de masa, comentado en el Capítulo 5. Las frecuencias de resonancia son ligeramente superiores a las establecidas por los estándares, para compensar la desviación que se producirá cuando se considere el efecto de la cubierta.

Las características de adaptación de esta antena se resumen en la Tabla 6.6.

Tabla 6.6: Características de adaptación de la antena de la antena "ring-patch" adaptada al terminal.

\begin{tabular}{|c|c|c|}
\hline Estándar & $\begin{array}{c}\text { Frecuencia central de } \\
\text { resonancia }\end{array}$ & $\begin{array}{c}\text { Ancho de banda } \\
\left.\mathbf{( S}_{\mathbf{1 1}}<-\mathbf{6} \mathbf{~ d B}\right)\end{array}$ \\
\hline $\mathrm{GSM}$ & $936 \mathrm{MHz}$ & $81 \mathrm{MHz}$ \\
$\mathrm{DCS}$ & $1822 \mathrm{MHz}$ & $182 \mathrm{MHz}$ \\
\hline
\end{tabular}




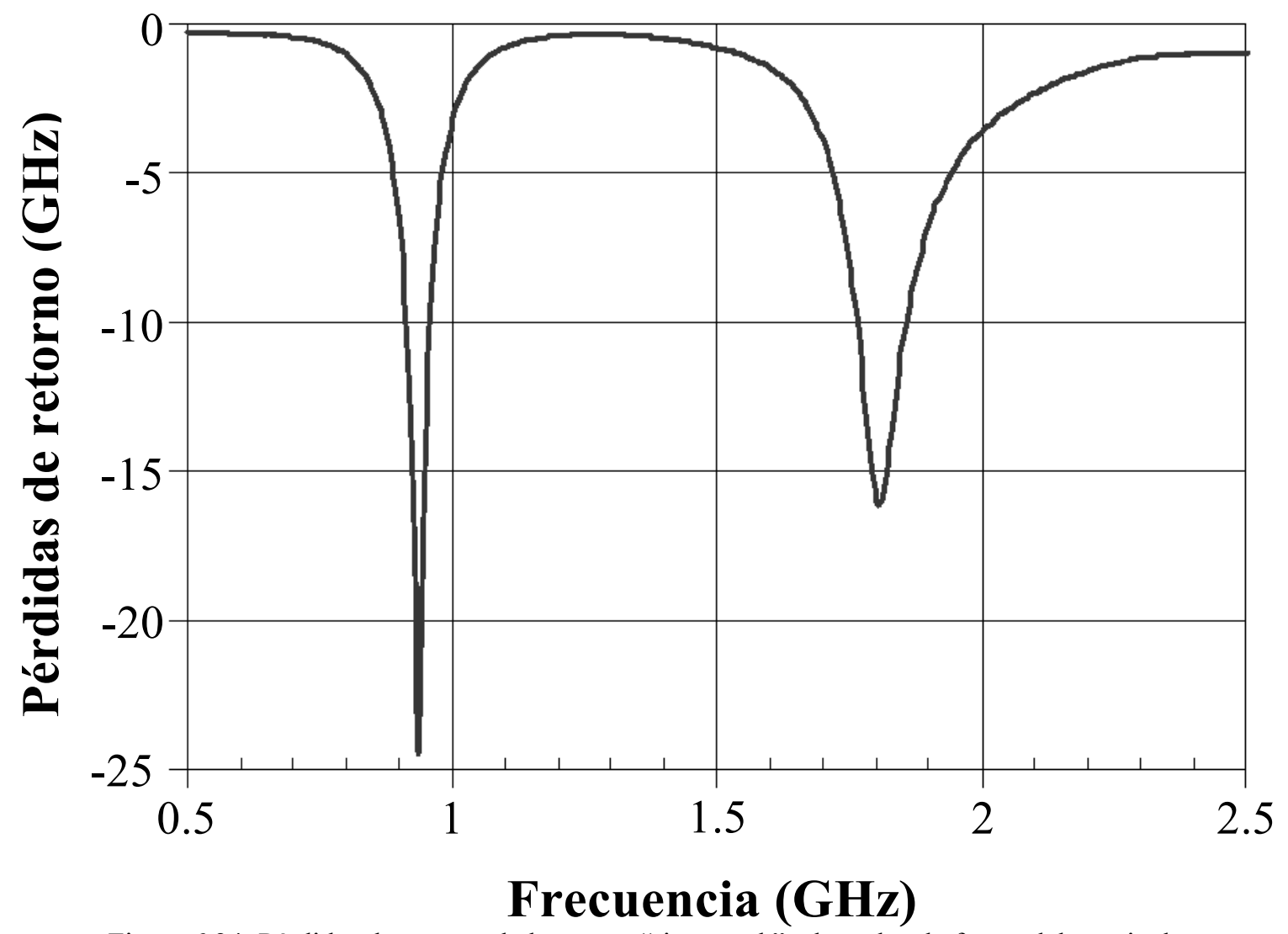

Figura 6.24: Pérdidas de retorno de la antena "ring-patch" adaptada a la forma del terminal.

El comportamiento de esta antena, en lo referente a campos próximos y diagramas de radiación, es prácticamente idéntico al de la antena "ring-patch" original presentada en el apartado 6.2.4.

\subsubsection{Antena conformada.}

Otro diseño de antena adaptada a la forma del terminal se muestra en la Figura 6.25. En este caso el PCB tenía una forma no rectangular, mientras que el espacio reservado a la antena era claramente irregular. Como en el caso anterior, también aquí hay estudiar, durante la fase de diseño, el efecto de determinados elementos situados en las proximidades del parche.

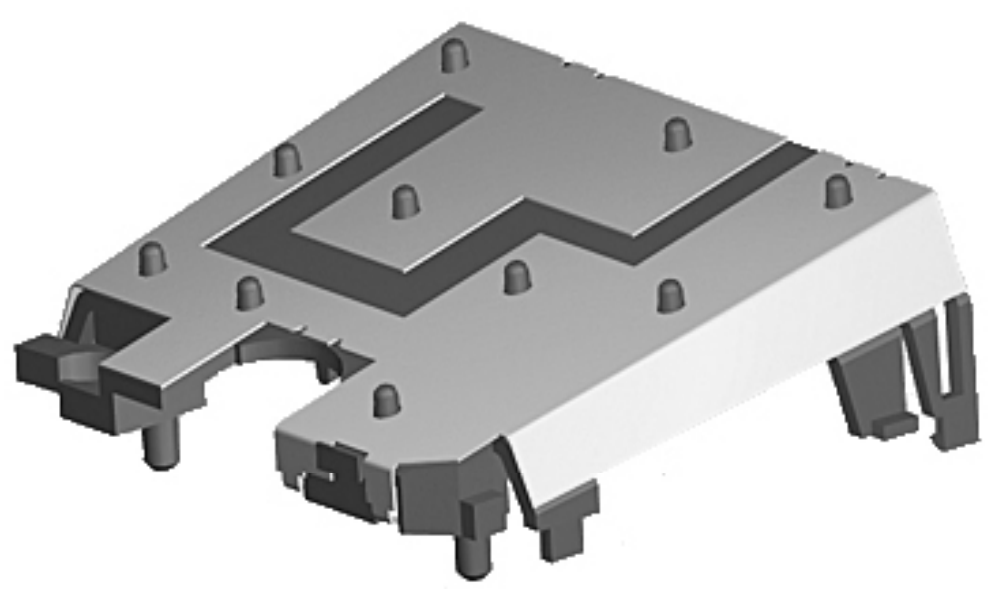

Figura 6.25: Esquema de construcción de la antena conformada. 
Para la fase de simulación hubo que recurrir al modelo de la Figura 6.26. Para mantenerse lo más cercanos posible del diseño definitivo se tuvo en cuenta la presencia de ciertos elementos, como circuitos apantallados bajo el parche, que reducían la altura efectiva de la antena sobre el plano de masa, y la batería.

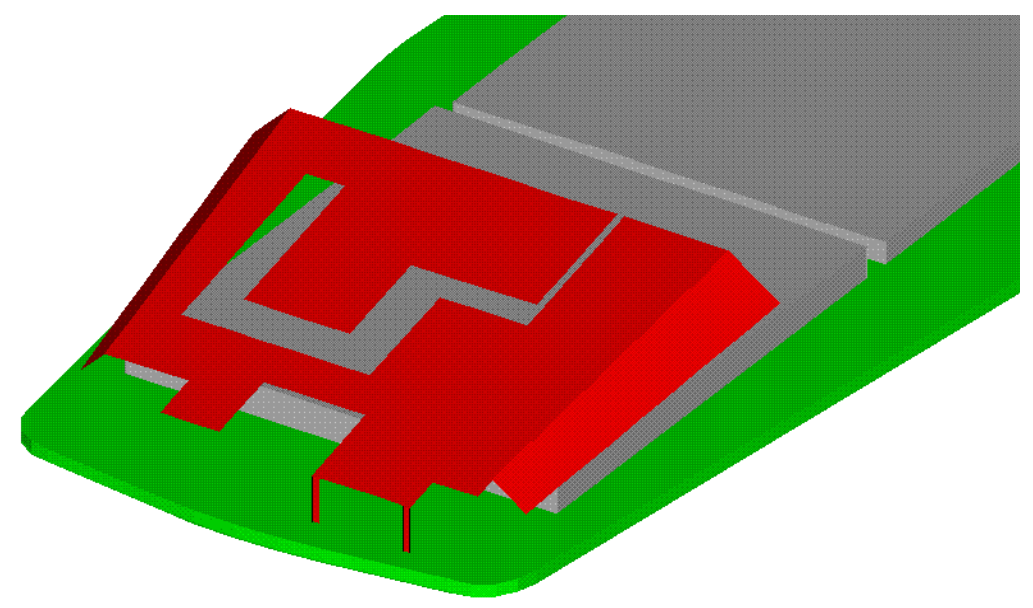

Figura 6.26: Modelo de simulación de una antena de doble banda adaptada a la cubierta del terminal.

La estructura definitiva de la antena, con su soporte mecánico, se muestra en la fotografía de la Figura 6.27.

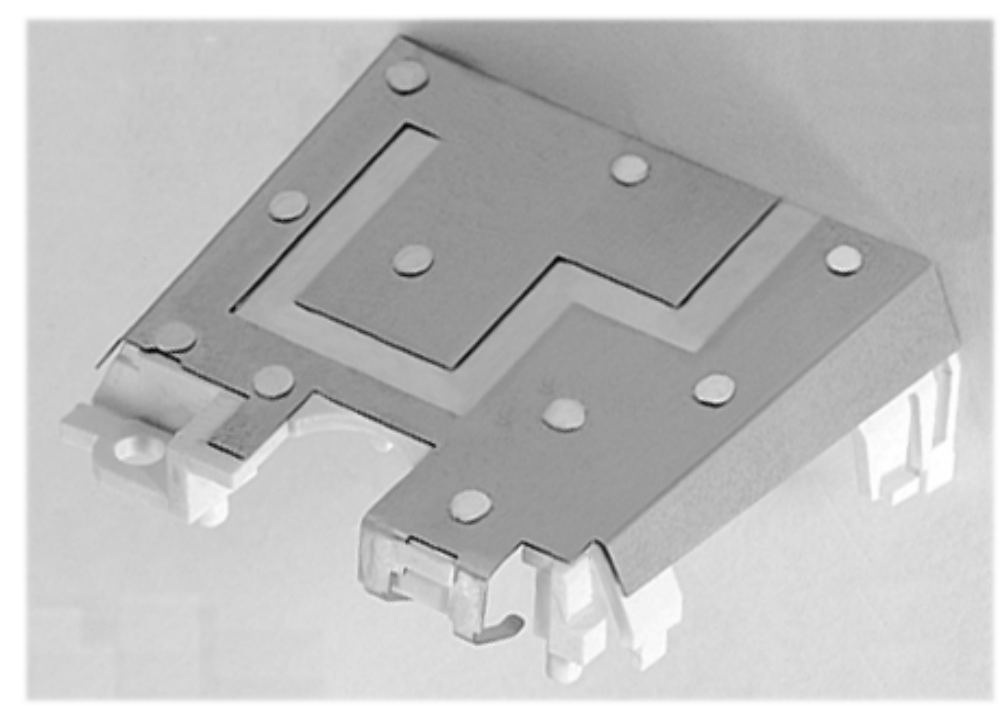

Figura 6.27: Antena sobre soporte de plástico.

En las curvas de la Figura 6.28 se aprecia cómo se consigue adaptar la antena a la forma del terminal sin degradar significativamente las características de adaptación. Las medidas se realizaron teniendo en cuenta tanto los componentes presentes en la pletina como la cubierta de plástico del terminal, cuyo efecto se discutirá con más detalle en el siguiente capítulo. 


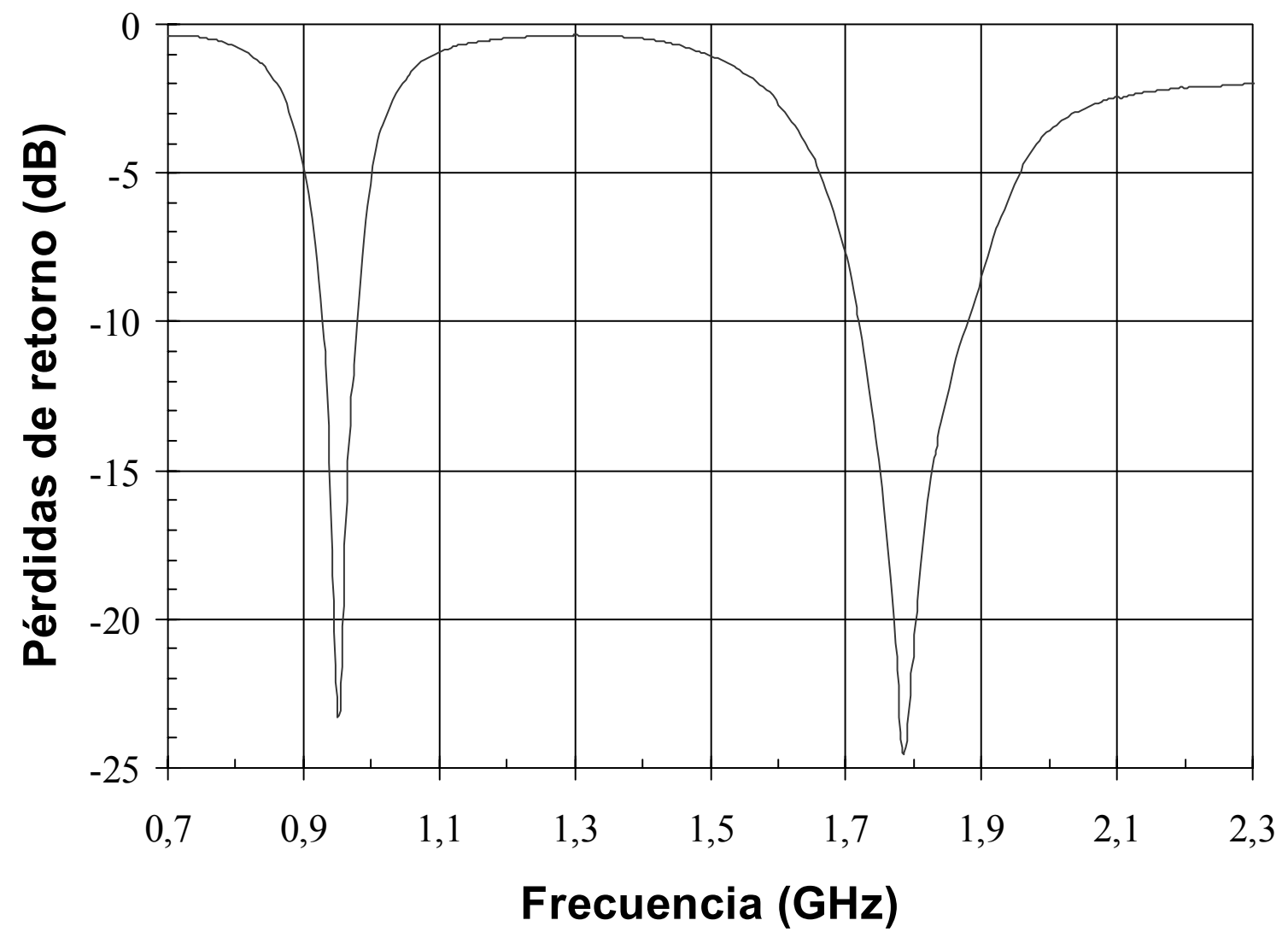

Figura 6.28: Pérdidas de retorno medidas de la antena de doble banda adaptada a la cubierta del terminal.

Las frecuencias de resonancia y los anchos de banda obtenidos, para unas pérdidas de retorno inferiores a $-6 \mathrm{~dB}$, se muestran en la Tabla 6.7. Para la banda de DCS, el ancho de banda es muy superior al mínimo exigido.

Tabla 6.7: Características de adaptación de la antena.

\begin{tabular}{|c|c|c|}
\hline Estándar & $\begin{array}{c}\text { Frecuencia central de } \\
\text { resonancia }\end{array}$ & $\begin{array}{c}\text { Ancho de banda } \\
\left.\mathbf{( S}_{\mathbf{1 1}}<-\mathbf{6} \mathbf{d B}\right)\end{array}$ \\
\hline $\mathrm{GSM}$ & $955 \mathrm{MHz}$ & $73 \mathrm{MHz}$ \\
$\mathrm{DCS}$ & $1787 \mathrm{MHz}$ & $216 \mathrm{MHz}$ \\
\hline
\end{tabular}

En la Tabla 6.8 se muestran los diagramas de radiación de la antena conformada, en los tres planos de corte principales y para las bandas de GSM y DCS, medidos según el sistema de coordenadas de la Figura 6.12.

La antena presenta una ganancia de $1,3 \mathrm{dBi}$ para la frecuencia central de la banda de GSM, y-0,7 dBi en la de DCS. Se puede apreciar que en el caso de DCS los diagramas son más irregulares, con una componente contrapolar más elevada, debido a la mayor influencia del PCB. 


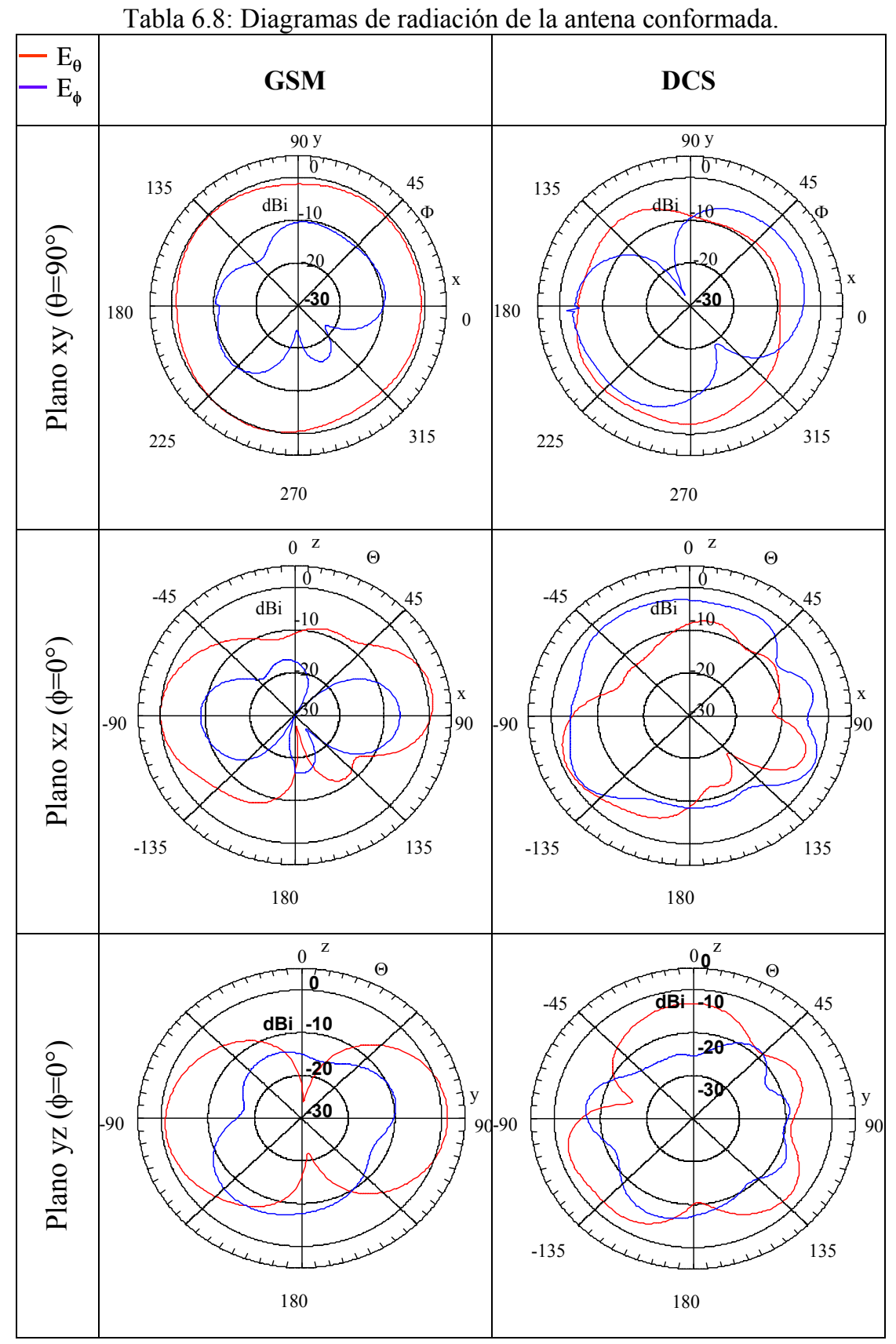

\subsection{ANTENAS DE TRES Y CUATRO BANDAS.}

Con el desarrollo de nuevos terminales y nuevos estándares, resulta necesario extrapolar los resultados del apartado anterior para poder cubrir bandas de frecuencias adicionales. Así, puede ser necesario el desarrollo de antenas para terminales que implementen diferentes combinaciones de estándares, como por ejemplo:

$\infty$ GSM/DCS/PCS

$\infty$ GSM/DCS/Bluetooth 


\section{\& GSM/DCS/UMTS \\ $\infty$ AMPS/PCS/GPS \\ $\infty$ AMPS/GSM/DCS/PCS \\ $\infty$ GSM/DCS/PCS/WLAN...}

En la mayoría de los casos se requiere la utilización de un único punto de alimentación. Así, es necesario excitar diferentes modos en el parche, que resonantes a la frecuencia central de las bandas de interés. Sin embargo, las bandas de frecuencias de algunos estándares se solapan, tal y como se puede apreciar en la Tabla 6.9 y la Figura 6.29.

Tabla 6.9: Bandas de frecuencias asignadas a los principales estándares (MHz)

\begin{tabular}{|c|c|c|}
\hline Estándar & Tx (Uplink) & Rx (Downlink) \\
\hline AMPS & $824-849$ & $869-894$ \\
\hline E-GSM & $880-915$ & $925-960$ \\
\hline DCS & $1710-1785$ & $1805-1880$ \\
\hline PCS1 & $1850-1910$ & $1930-1990$ \\
\hline UMTS FDD & $1920-1980$ & $2110-2170$ \\
\hline UMTS TDD2 & $1900-1920 / 2010-2025$ \\
\hline DECT & \multicolumn{2}{|c|}{$1880-1900$} \\
\hline ISM & \multicolumn{2}{|c|}{$2400-2500$} \\
\hline Bluetooth & \multicolumn{2}{|c|}{$2400-2500$} \\
\hline WLAN & $1570-1580$ & 1575,2 \\
\hline GPS & \multicolumn{2}{|c|}{} \\
\hline
\end{tabular}

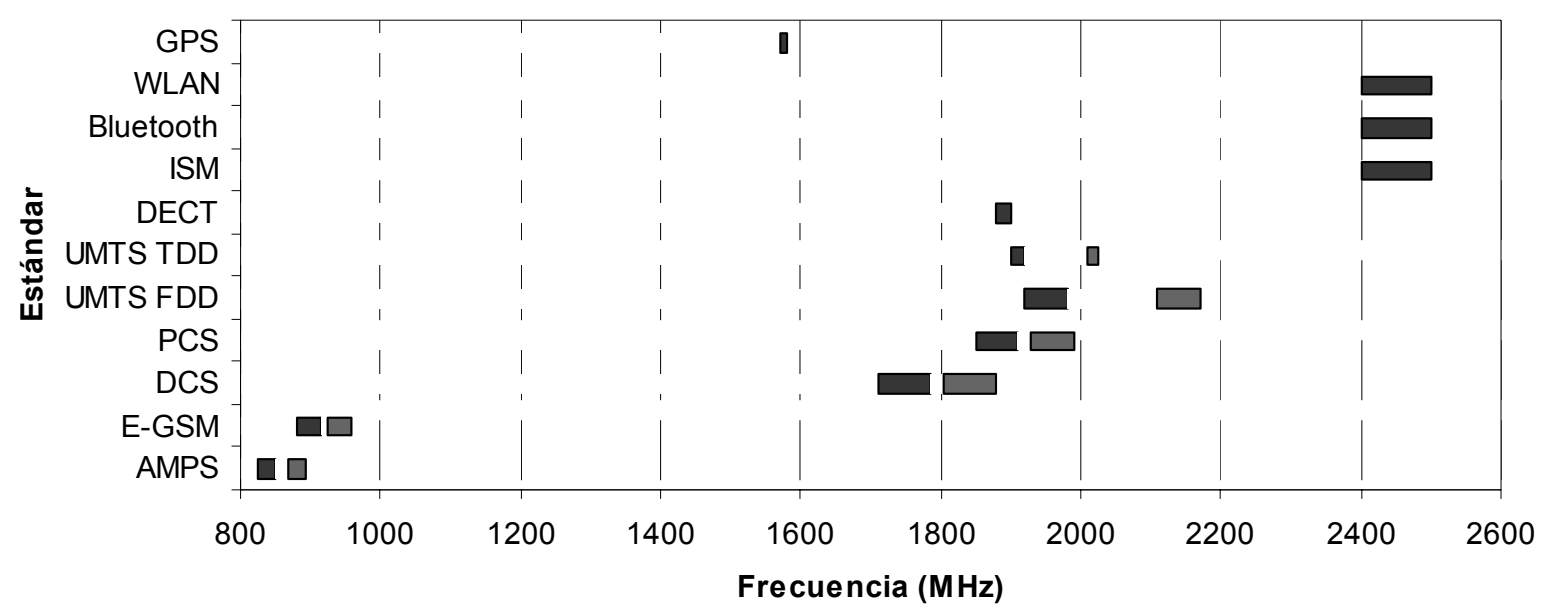

Figura 6.29: Distribución de las bandas de frecuencia (en MHz) de los principales estándares.

\footnotetext{
${ }^{1}$ En los EE.UU. la banda de PCS está destinada también a UMTS.

${ }^{2}$ En UMTS TDD las dos bandas asignadas se utilizan tanto para emisión como para recepción
} 
Para poder cubrir dos estándares con bandas que se solapan existen dos alternativas: tratar de excitar un único modo con un ancho de banda suficiente, o dos modos acoplados entre sí. En este último caso resulta importante controlar perfectamente el mecanismo de acoplamiento, puesto que cualquier modificación de la geometría del parche puede significar una pérdida importante de ancho de banda.

Para implementar estas antenas integradas multibanda se suele recurrir a estructuras que incluyen elementos parásitos, y a combinaciones de parches y ranuras radiantes. En cualquier caso, el principal problema reside en cómo realizar ajustes en una de las bandas sin por ello interferir en el funcionamiento de las demás, puesto que debido a los acoplamientos mutuos la antena es muy sensible a los cambios.

Por otra parte, es importante tener en cuenta el efecto del dieléctrico utilizado en la fabricación del soporte de la antena. Este efecto se discutirá en el capítulo siguiente.

\subsubsection{Antena de triple banda con parche parásito M3.}

Para poder cumplir los estándares de segunda generación GSM, DCS y PCS se necesitan antenas resonantes a tres frecuencias diferentes. Dado que las bandas de DCS y PCS se solapan, también es posible recurrir a antenas duales, y procurar ensanchar la banda superior mediante el uso, por ejemplo, de parches parásitos cortocircuitados coplanares [6.6], [6.7] o a diferente altura [6.8] con respecto al parche principal.. Sin embargo, si su utilización implica normalmente un aumento del tamaño total de la estructura, lo que puede acarrear problemas de espacio dentro del terminal.

En primer lugar se analizará una antena de triple banda derivada de la antena dual presentada en el apartado 6.2.2. Se trata de la combinación entre una PIFA de doble banda con un elemento parásito en $\lambda / 4$ cortocircuitado. Con la presencia del parche parásito, aparece un nuevo modo resonante, debido al acoplamiento entre los diferentes elementos, que permite cubrir la banda de PCS [6.9]. La forma de la antena se puede apreciar en la Figura 6.30.

La superficie de la estructura es de $39 \mathrm{~mm}$ x $26 \mathrm{~mm}$, con una altura de $8 \mathrm{~mm}$ sobre el plano de masa. En este caso, se modificó la estructura del parche original, para mantener la misma longitud eléctrica y por lo tanto las frecuencias de resonancia iniciales. El parche parásito se puede incluir así sin por ello incrementar el tamaño total de la estructura con respecto al de la antena de banda dual.

Para garantizar la estabilidad mecánica a la estructura de cara a las medidas se utilizó un bloque de Styropor situado bajo los parches, tal y como se muestra en la Figura 6.31. En la práctica, éste deberá ser sustituido por un soporte de material plástico, cuyo efecto se estudiará más adelante. 


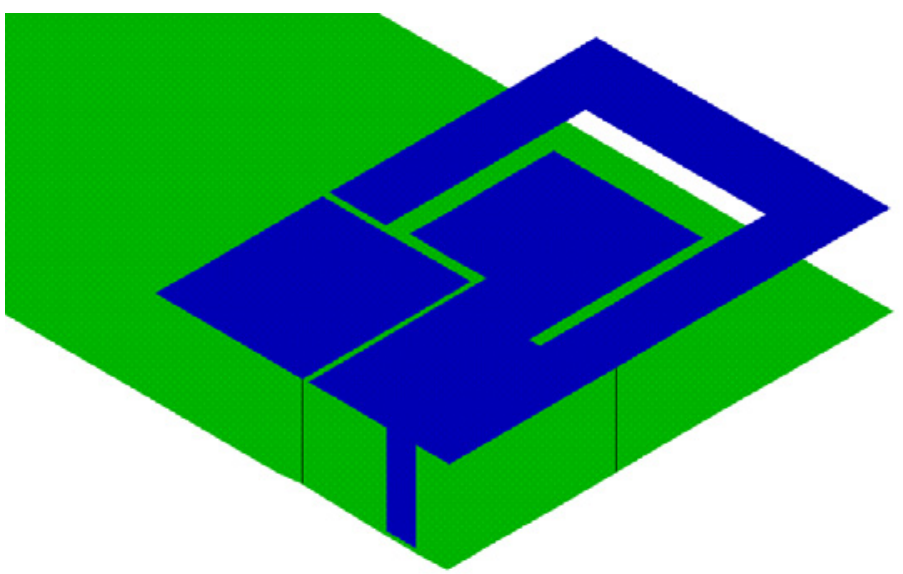

Figura 6.30: Estructura de la antena de triple banda M3 con parche parásito cortocircuitado.

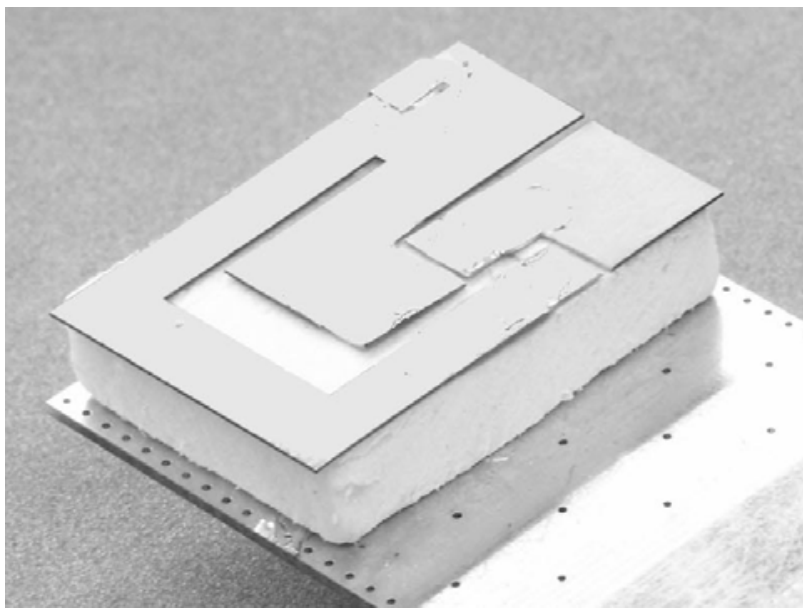

Figura 6.31: Antena M3.

En la Figura 6.32 se recogen los resultados de las pérdidas de retorno medidas. Se puede observar claramente una doble resonancia para una frecuencia de unos $2 \mathrm{GHz}$, que permitiría cubrir las bandas de DCS y PCS, además de la banda de GSM. Las medidas se llevaron a cabo teniendo en cuenta la cubierta de plástico del terminal, que introduce una cierta desviación en las frecuencias de resonancia que se debe considerar al realizar el diseño.

En la Tabla 6.10 se recogen las características de adaptación de la antena. En este caso se toma como referencia para el ancho de banda unas pérdidas de retorno de $-7,5 \mathrm{~dB}$. Se puede apreciar cómo los anchos de banda son suficientes para cubrir los estándares considerados. Se observa sin embargo una desintonización de las frecuencias de resonancia, que se podría compensar con relativa facilidad realizando pequeños ajustes en el tamaño del parche.

Tabla 6.10: Características de adaptación de la antena de triple banda M3.

\begin{tabular}{|c|c|c|}
\hline Estándar & $\begin{array}{c}\text { Frecuencia central de } \\
\text { resonancia }\end{array}$ & $\begin{array}{c}\text { Ancho de banda } \\
\mathbf{( S}_{\mathbf{1 1}}<-7,5 \\
\mathbf{~ d B})\end{array}$ \\
\hline $\mathrm{GSM}$ & $1004 \mathrm{MHz}$ & $133 \mathrm{MHz}$ \\
$\mathrm{DCS} / \mathrm{PCS}$ & $1863 / 2056 \mathrm{MHz}$ & $216 \mathrm{MHz}$ \\
\hline
\end{tabular}




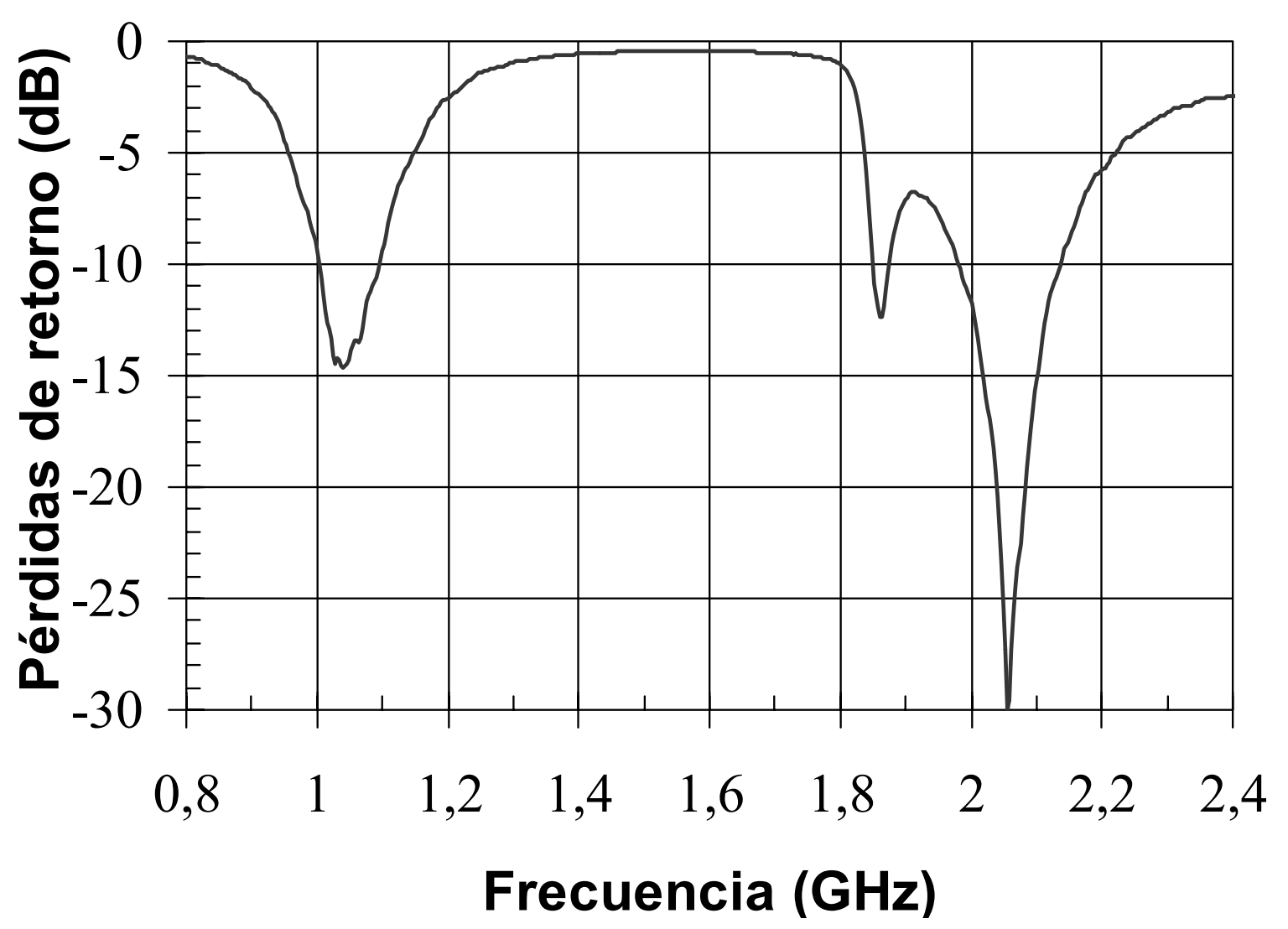

Figura 6.32: Pérdidas de retorno medidas de la antena de triple banda M3.

Las distribuciones de campos para las diferentes bandas de frecuencia se muestran en la Figura 6.33, la Figura 6.34 y la Figura 6.35. En la primera se observa cómo el modo correspondiente a GSM se debe a la resonancia a lo largo de la rama externa del parche, como en el caso de la antena M de doble banda.

La banda de DCS se cubre utilizando la resonancia de la parte central del parche principal, mientras que para la de PCS se recurre al efecto del parche parásito, y su acoplamiento con el resto de la estructura.
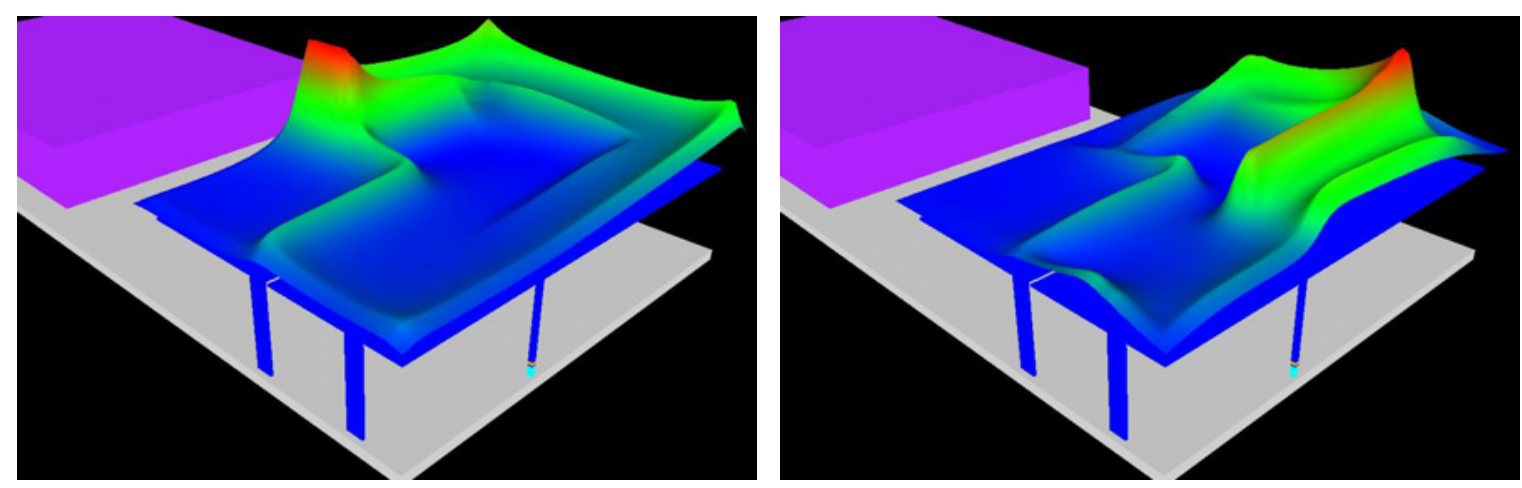

Figura 6.33: Distribución de campo eléctrico (izquierda) y magnético (derecha) de la antena M3 en la banda de GSM. 

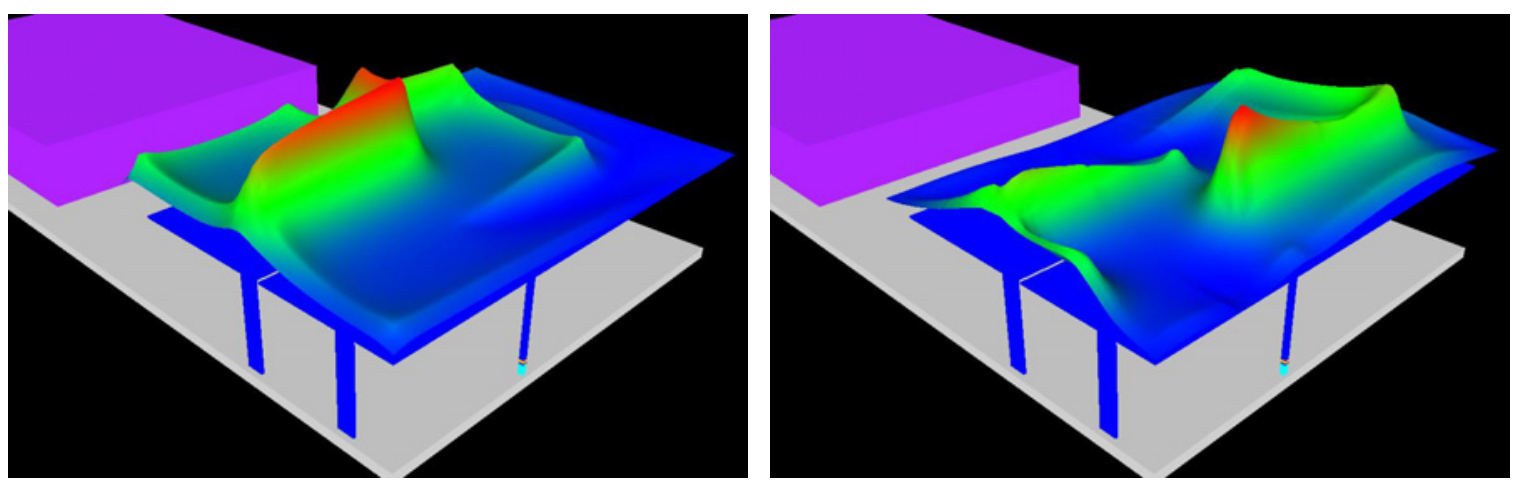

Figura 6.34: Distribución de campo eléctrico (izquierda) y magnético (derecha) de la antena M3 en la banda
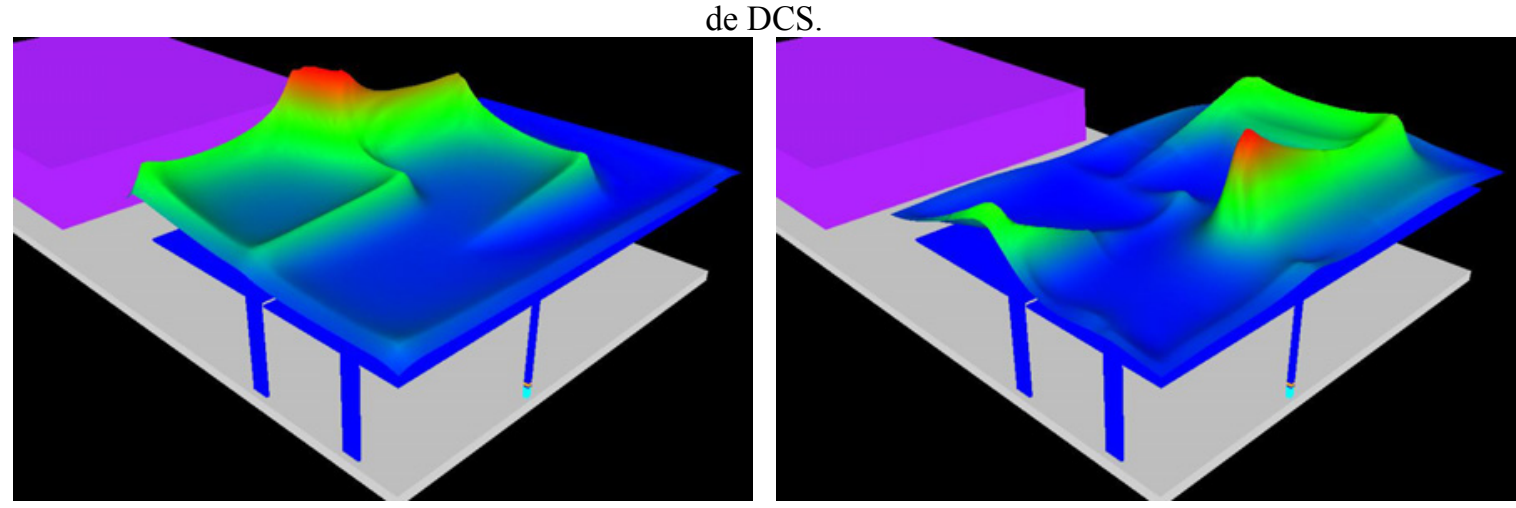

Figura 6.35: Distribución de campo eléctrico (izquierda) y magnético (derecha) de la antena M3 en la banda de PCS.

Los diagramas de radiación de la antena, presentados en la Figura 6.36, son análogos a los de la antena M para las frecuencias de GSM y PCS. El de la banda de DCS presenta, sin embargo, una deformación debida al efecto del parche parásito.
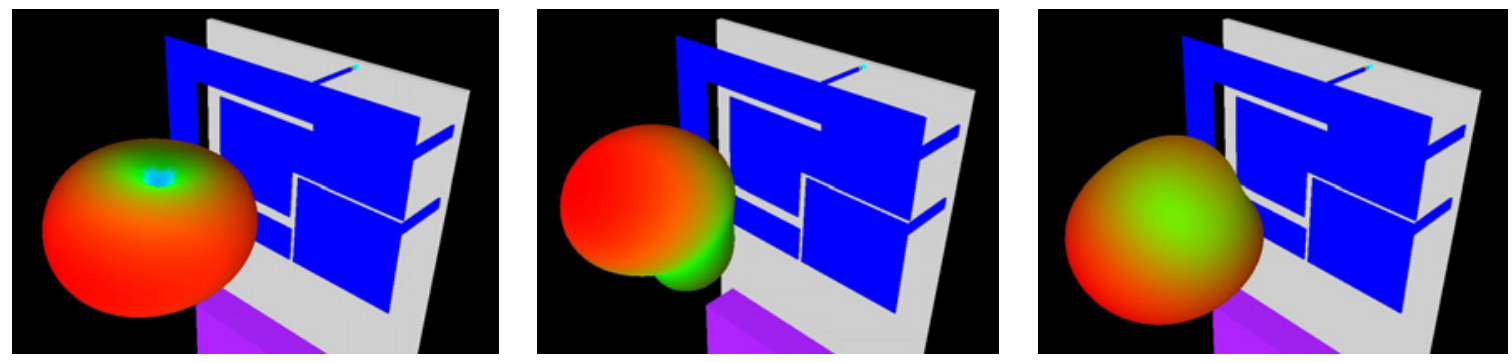

Figura 6.36: Diagrama de radiación de la antena M3 en las bandas de GSM (izquierda), y DCS (centro) y PCS (derecha).

\subsubsection{Antena de triple banda MT.}

Tal y como se ha mencionado anteriormente, el espacio destinado a la antena en el interior de un terminal es cada vez menor. Se desarrolló por lo tanto una versión de la antena presentada en el apartado 6.4.1, con un tamaño menor que la original. Para ello hubo que reducir el ancho de los elementos radiantes y del parche parásito, así como la distancia entre los diferentes elementos. Con ello, aumenta el acoplamiento capacitivo entre las diferentes partes del parche, y se consigue pues reducir el tamaño total de la estructura. Sin embargo al hacerlo se pierde ancho de banda, aunque 
éste sigue siendo suficiente para cumplir las especificaciones establecidas hoy en día por los fabricantes.

La antena fue diseñada con una superficie de $16 \mathrm{~mm} \times 36 \mathrm{~mm}$, con una altura de $8 \mathrm{~mm}$ sobre un plano de masa de $36 \mathrm{~mm} \times$ 95mm, tal y como se muestra en la Figura 6.37 [6.10].

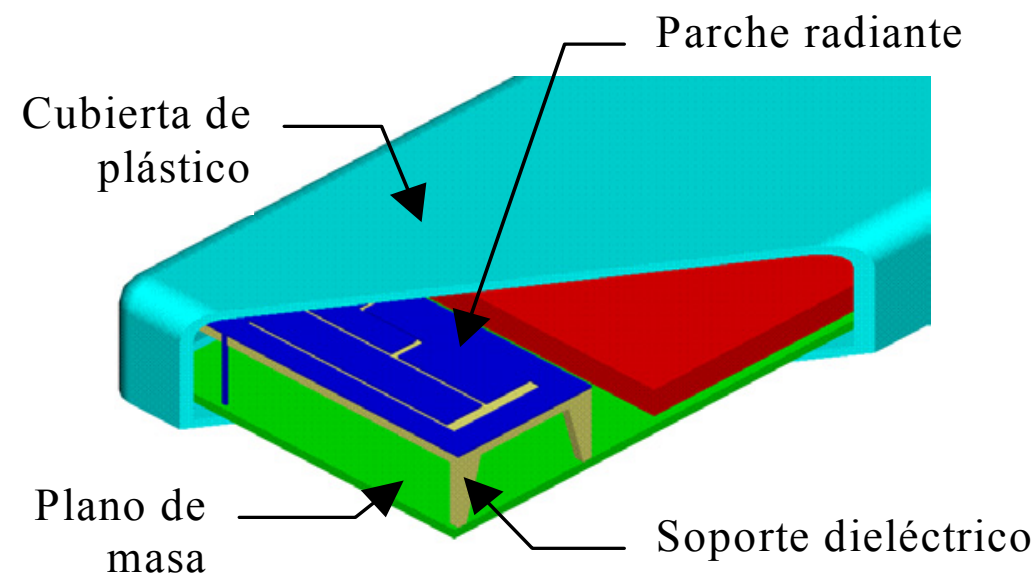

Figura 6.37: Modelo de terminal con antena integrada de triple banda MT.

La geometría de la antena se puede observar con más detalle en la fotografía de la Figura 6.38. Para garantizar la estabilidad de la estructura, sin alterar significativamente la permitividad efectiva, se colocó un bloque de Styropor bajo el parche.

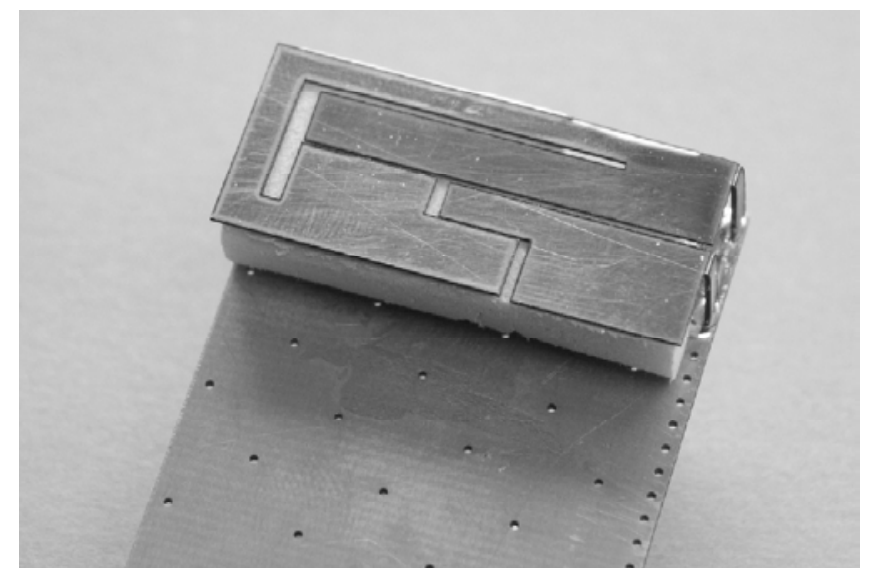

Figura 6.38: Antena de triple banda MT.

En la Figura 6.39 se puede apreciar cómo los resultados obtenidos por simulación utilizando el MoM se ajustan a los obtenidos en la realidad. En ambos casos se aprecia claramente la doble resonancia que permite cubrir las bandas de GSM y de PCS. Las frecuencias de resonancia se encuentran por encima de las establecidas en los estándares, para compensar la desviación que introducirá la cubierta de plástico en el diseño final. Los resultados obtenidos en las medidas se presentan en la Tabla 6.11 . 
Tabla 6.11: Características de adaptación de la antena de triple banda MT.

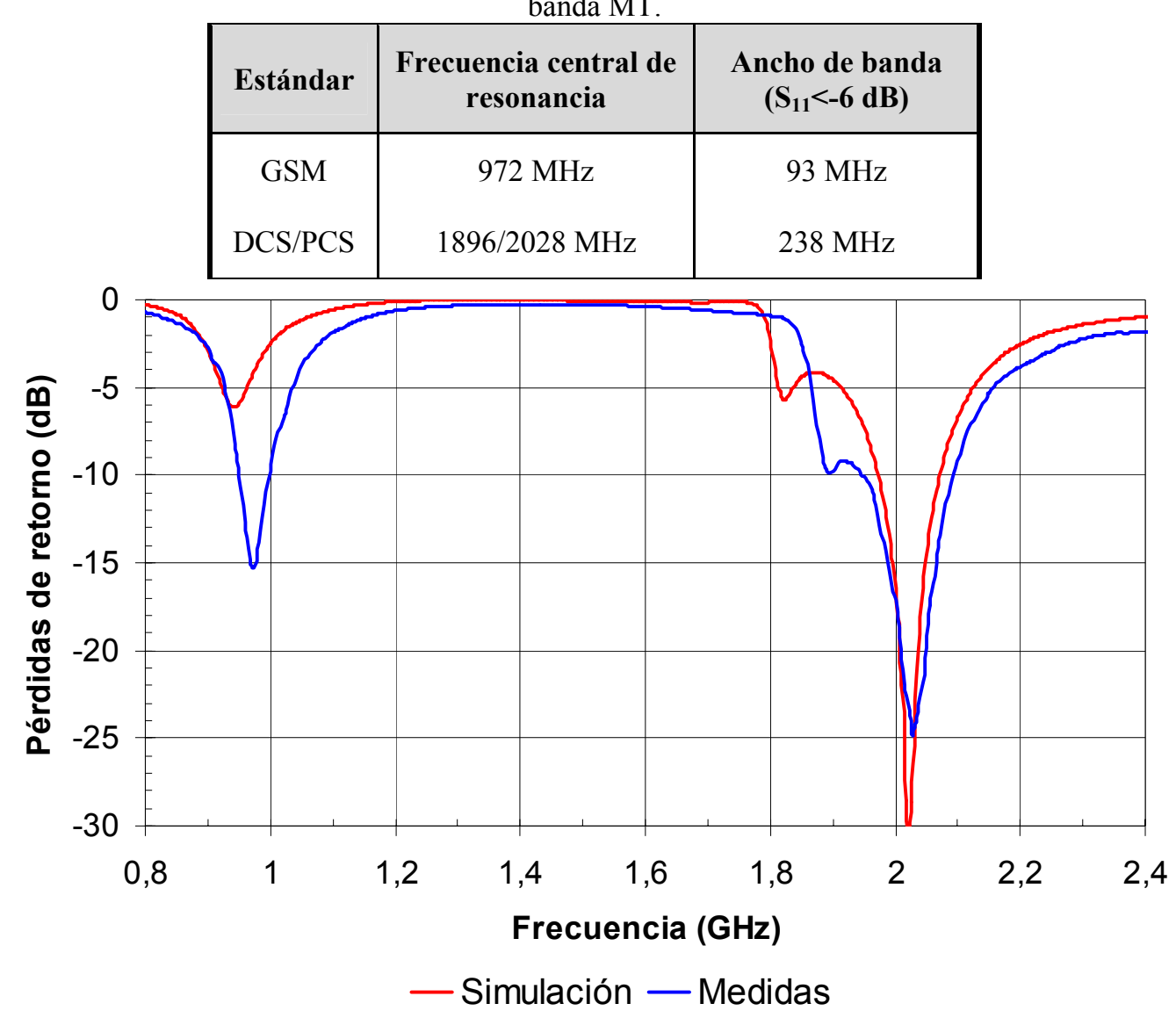

Figura 6.39: Simulación y medidas de las pérdidas de retorno de la antena de triple banda MT.

De la Figura 6.40 a la Figura 6.42 se representan las distribuciones de campo en un plano próximo a la superficie de la antena. Se observa cómo la rama exterior del parche sigue siendo la responsable del modo de GSM. El acoplamiento entre esta rama, la parte central del parche principal y el parche parásito permite generar una doble resonancia para cubrir DCS y PCS. El pin de alimentación se ensancha en su parte superior para mejorar la impedancia de entrada de la antena en todas las bandas de frecuencias.
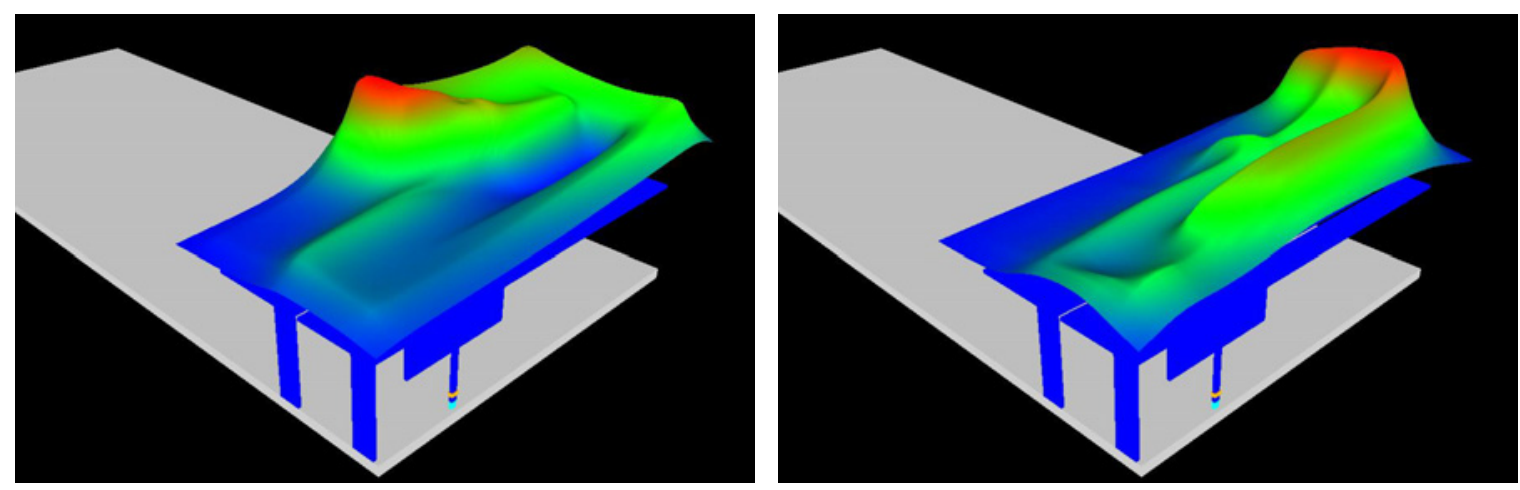

Figura 6.40: Distribución de campo eléctrico (izquierda) y magnético (derecha) de la antena MT en la banda de GSM. 

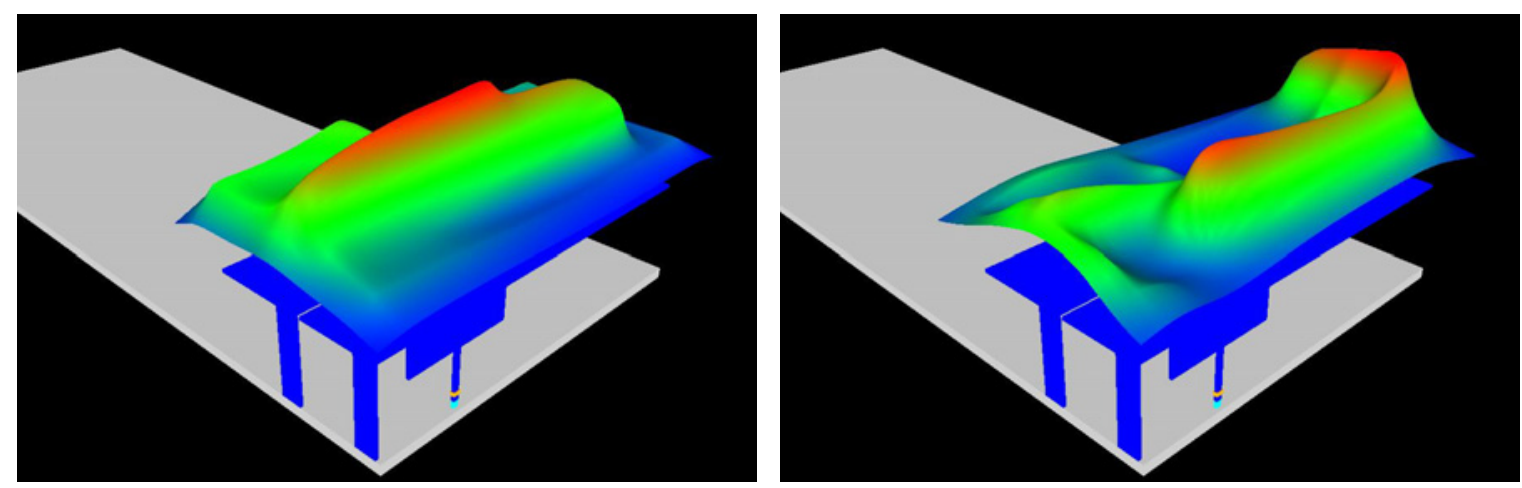

Figura 6.41: Distribución de campo eléctrico (izquierda) y magnético (derecha) de la antena MT en la banda

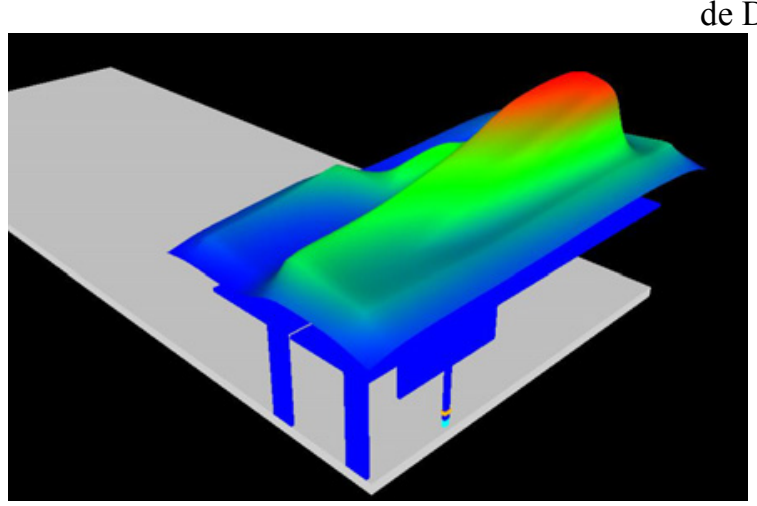
de DCS.

Figura 6.42: Distribución de campo eléctrico (izquierda) y magnético (derecha) de la antena MT en la banda de PCS.

En este caso, como el tamaño total de la estructura es mucho menor que en el caso de la antena M3, los acoplamientos son más fuertes, y el comportamiento de la antena mucho más sensible a las tolerancias. Para evitar problemas en cuanto a tolerancias en el proceso de fabricación, puede resultar conveniente imprimir la antena directamente sobre una lámina de material dieléctrico, como por ejemplo Teflón.

Los diagramas de radiación de la antena se muestran en la Figura 6.43. Debido a la reducción del tamaño del parche, la forma de dichos diagramas se modifica ligeramente con respecto a la obtenida con la antena M3 en las bandas de PCS y DCS. En GSM, sin embargo, el comportamiento sigue siendo análogo al del dipolo, debido a la influencia de la pletina.
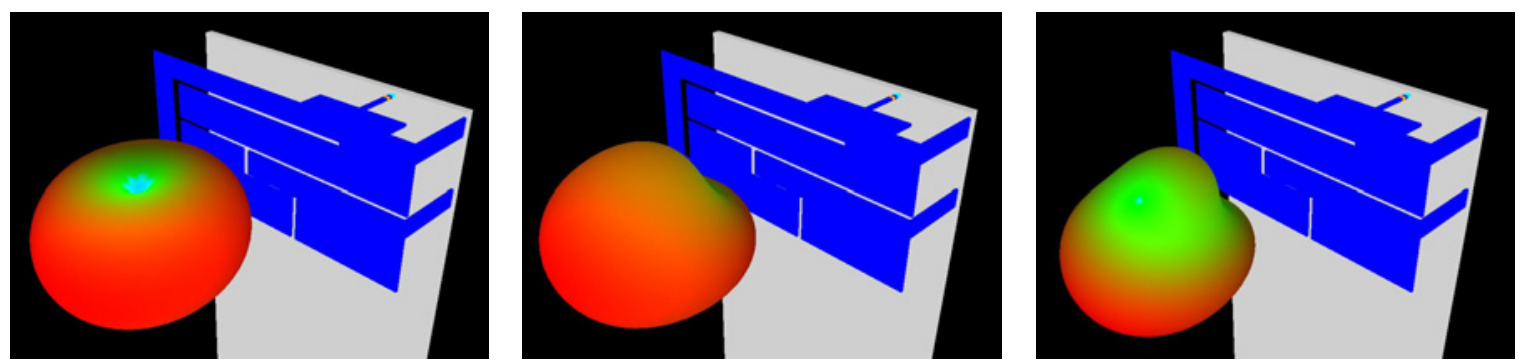

Figura 6.43: Diagrama de radiación de la antena MT en las bandas de GSM (izquierda), y DCS (centro) y PCS (derecha).

Los diagramas de radiación medidos se recogen en la Tabla 6.12. En este caso se ha considerado únicamente el campo eléctrico total en los planos de corte principales para la frecuencia central de 
cada una de las bandas consideradas. Se puede observar cómo todos los diagramas presentan unas buenas características en el plano acimutal (plano xy), siendo casi omnidireccional en el caso de GSM. En los otros planos de corte, se puede ver cómo en GSM se reproduce el comportamiento del dipolo, mientras que para DCS y PCS las características son similares a las de las antenas impresas sobre un plano de masa finito, con lóbulos debidos al efecto de la pletina. Las ganancias medidas son 0,5 dBi en la banda de GSM, 0,1 dBi en la de DCS y 2,7 dBi para PCS.

Tabla 6.12: Diagramas de radiación medidos de la antena MT.

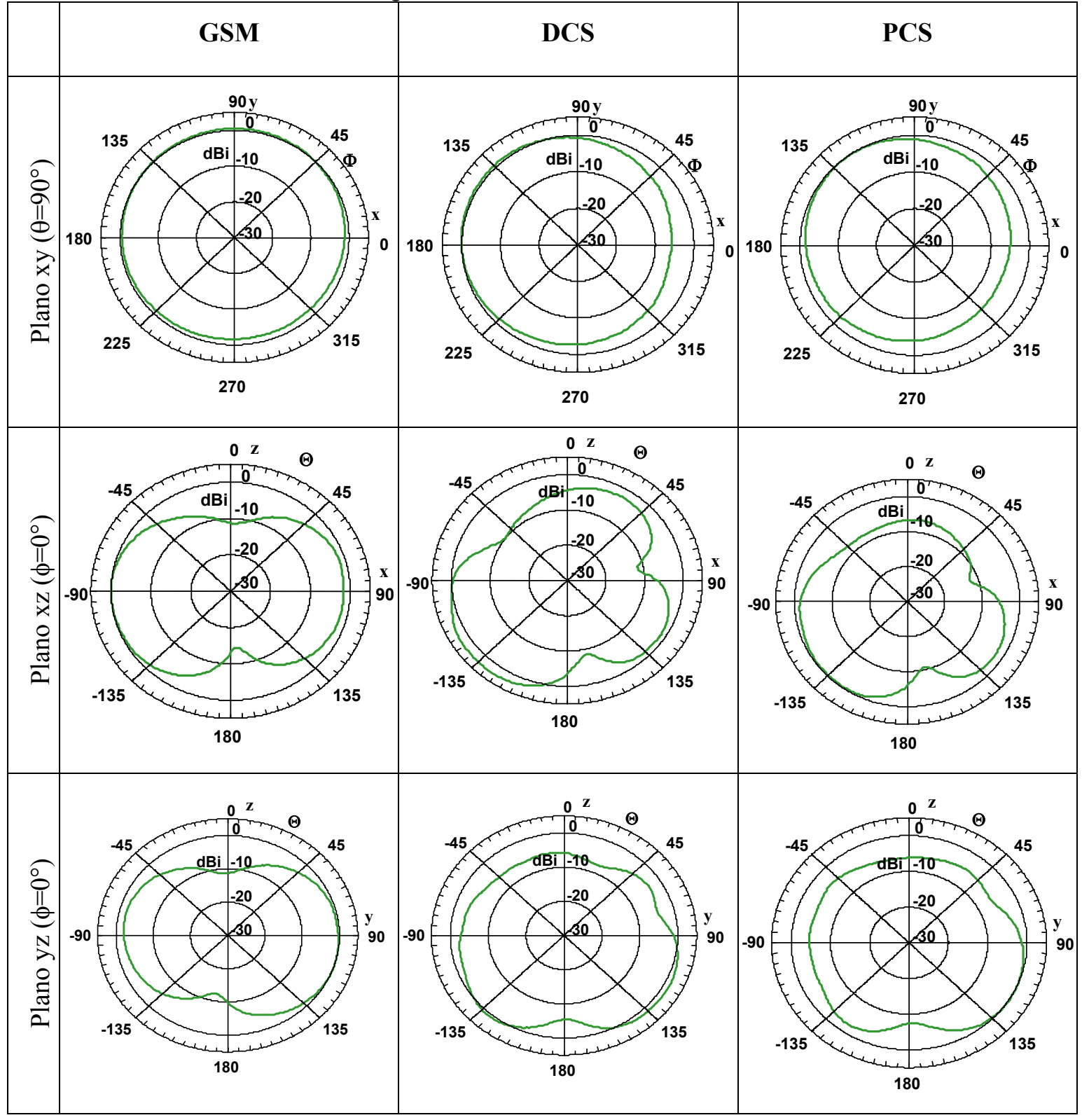

\subsubsection{Antena de cuatro bandas $M Q$.}

Si además de las bandas de GSM, DCS y PCS se pretende cubrir la banda de UMTS, hay que buscar una alternativa para añadir un nuevo modo resonante sin incrementar las dimensiones totales de la estructura. La mejor opción consiste en recortar una ranura o slot en el parche radiante, 
como por ejemplo los presentados en [6.3] para obtener una operación dual.

Así, se puede considerar la opción de añadir una ranura radiante a la antena del apartado 6.4.2. Dicha ranura se inserta en el perímetro de la antena, y tiene una longitud aproximada de $\lambda / 2$ a la frecuencia de UMTS. De esta forma se obtiene una antena de cuatro bandas sin aumentar el tamaño de la estructura, tal y como se muestra en la Figura 6.44 [6.11]. El ancho de la ranura está limitado, por una parte, por el ancho del parche en el que se inserta, en este caso $3 \mathrm{~mm}$, y por otra, por criterios tecnológicos, ya que no se podían recortar ranuras de menos de $0,2 \mathrm{~mm}$. Así, con un ancho de $0,5 \mathrm{~mm}$ se logra que la ranura entre en resonancia, sin por ello perturbar el modo de GSM excitado en esa rama del parche.

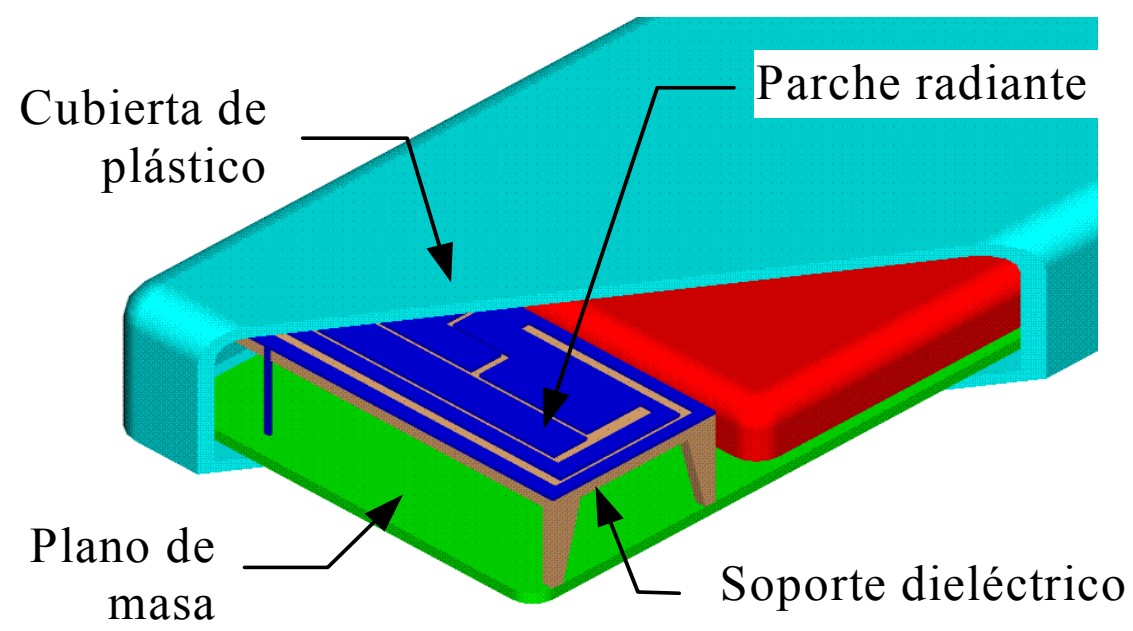

Figura 6.44: Modelo de la antena de cuatro bandas MQ integrada en el terminal.

Debido a la inestabilidad de la estructura metálica, se utilizó de nuevo un bloque de Styropor para asegurar la estabilidad de la estructura. Una foto de la antena puede verse en la Figura 6.45.

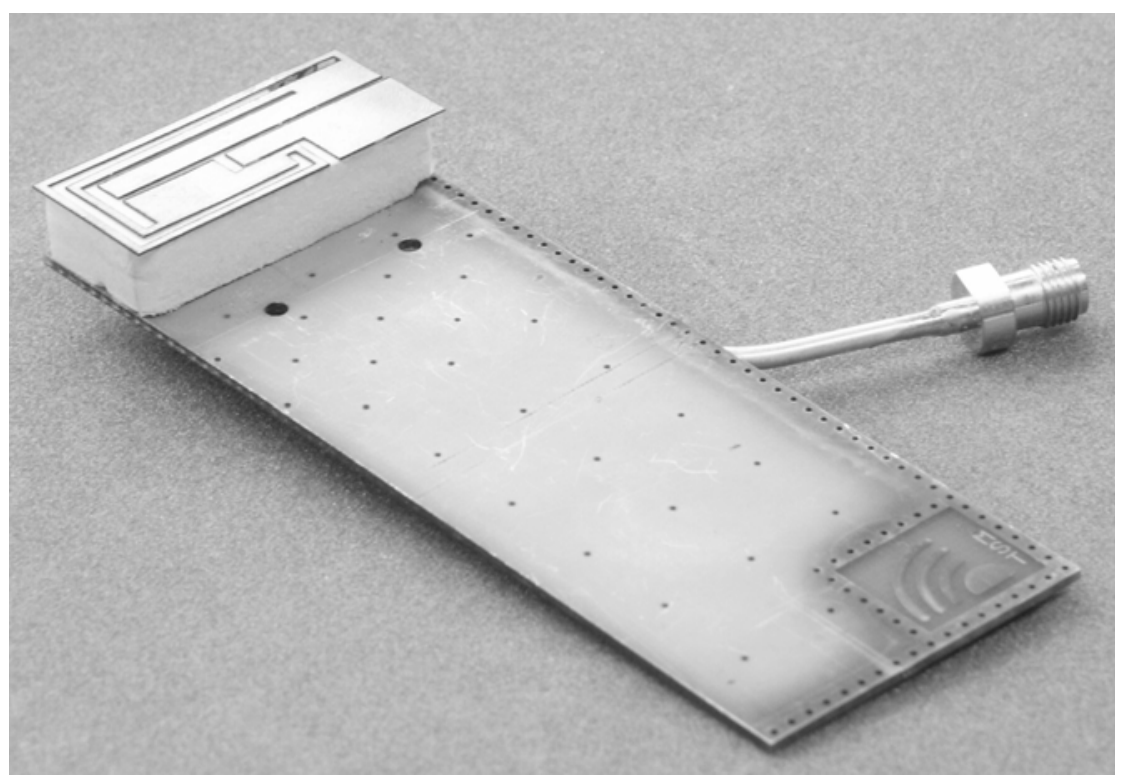

Figura 6.45: Antena MQ.

En la Figura 6.46 se muestra una comparación entre las pérdidas de retorno simuladas utilizando diferentes programas, y las obtenidas en la práctica. Utilizando simuladores 3D basados en el MoM 
y en la FDTD, presentados en el Capítulo 4, se obtuvo una buena predicción de la frecuencia de resonancia de GSM, DCS y PCS. Sin embargo se observa un desplazamiento en frecuencia para la banda de UMTS, correspondiente al modo de la ranura [6.12].

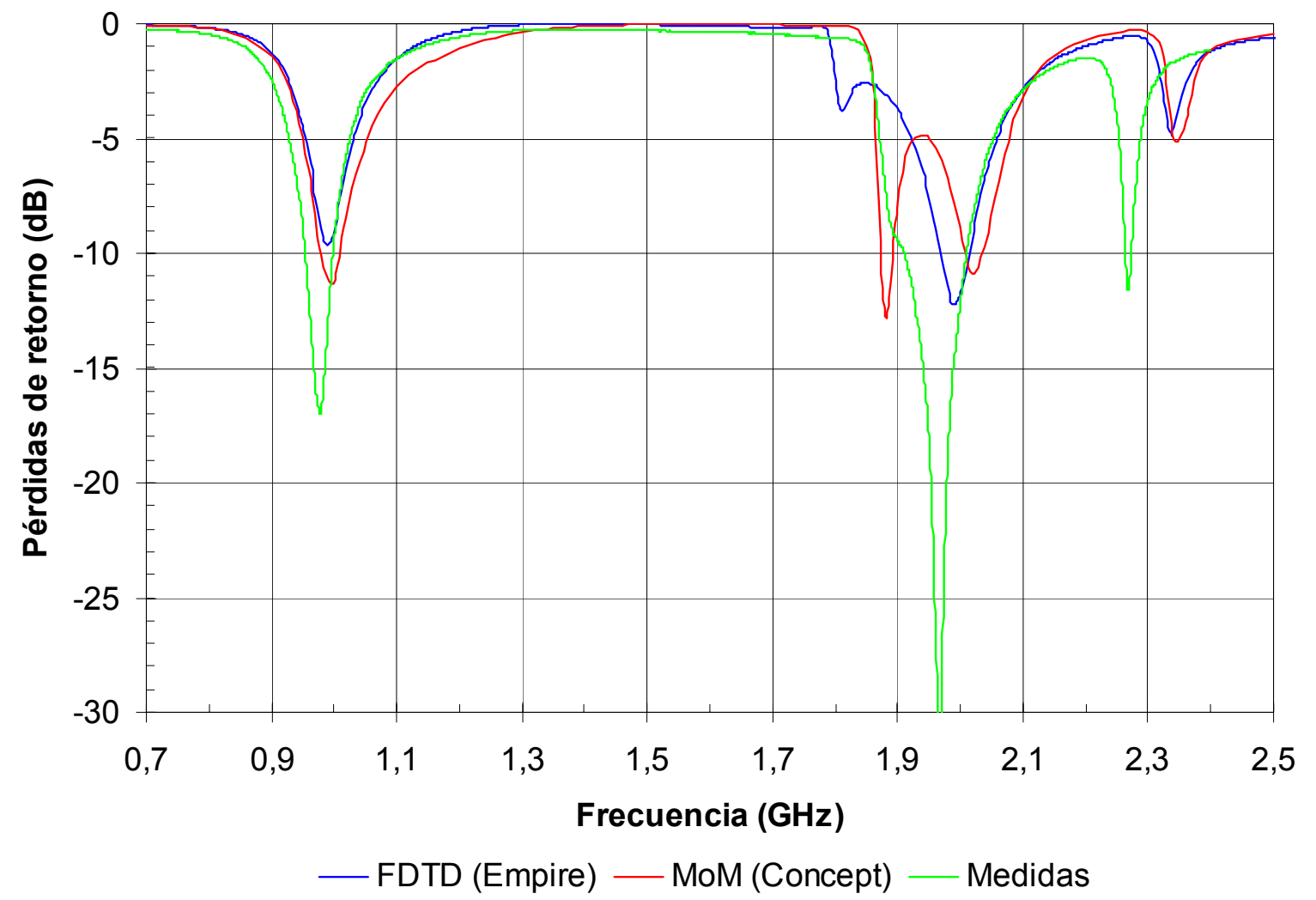

Figura 6.46: Simulación y medidas de la antena de cuatro bandas MQ.

De la Figura 6.47 a la Figura 6.50 se muestran las distribuciones de campo eléctrico para los diferentes modos excitados en la antena.

Los tres primeros, correspondientes a las bandas de GSM, DCS y PCS, no varían significativamente con respecto a la antena MT del apartado 6.4.2. Se aprecia únicamente el efecto de la ranura en el brazo que genera la resonancia de GSM, en la Figura 6.47. Sin embargo el acoplamiento capacitivo con el resto del parche no sufre apenas modificaciones, por lo que el comportamiento de la antena será similar al de la antena MT.
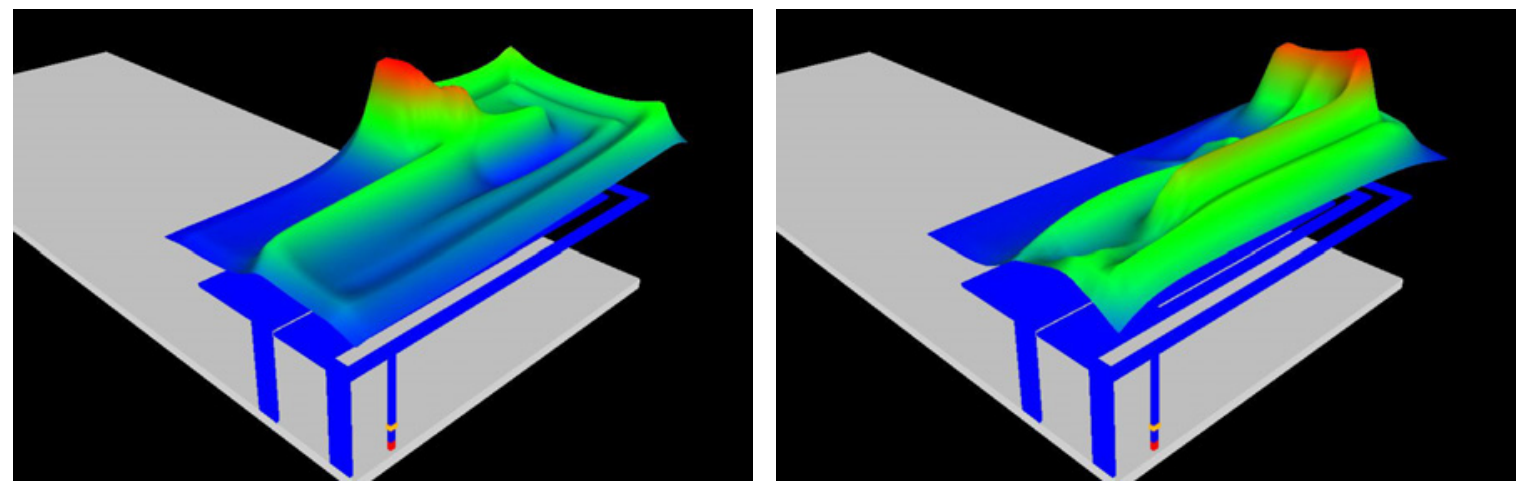

Figura 6.47: Distribución de campo eléctrico (izquierda) y magnético (derecha) de la antena MQ en la banda de GSM. 

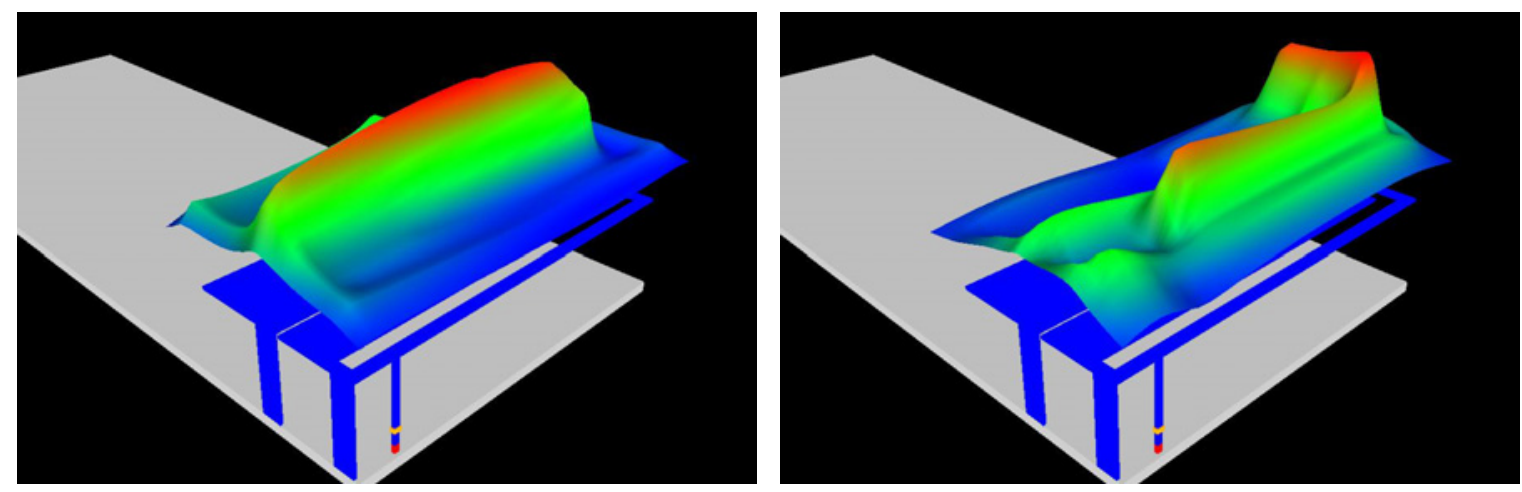

Figura 6.48: Distribución de campo eléctrico (izquierda) y magnético (derecha) de la antena MQ en la banda de DCS.
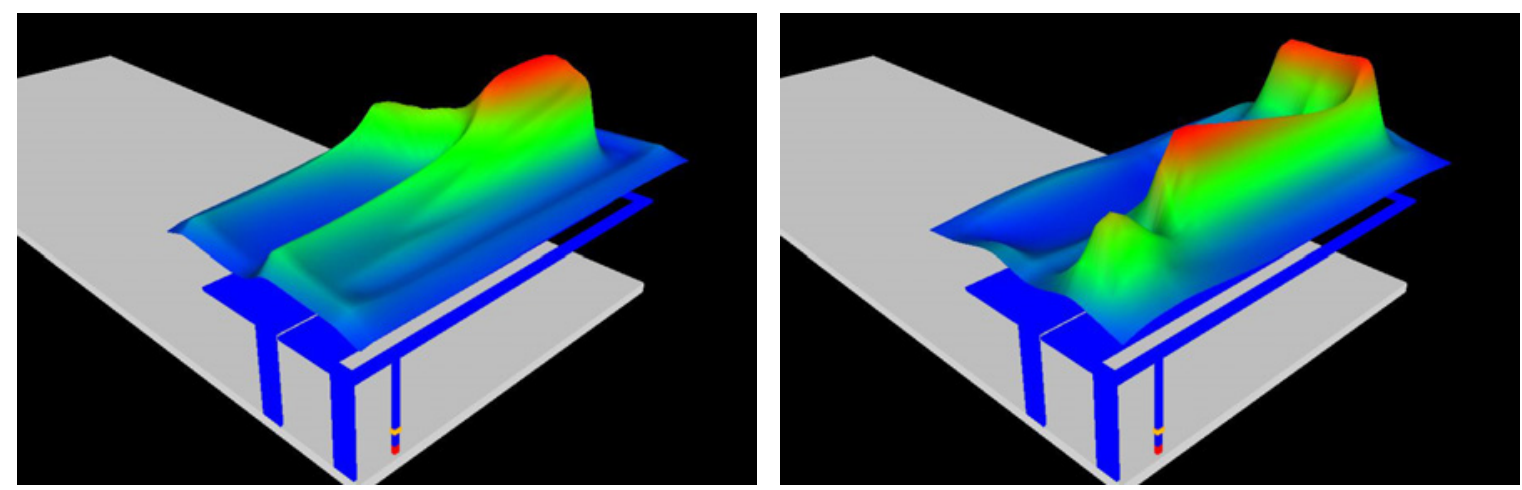

Figura 6.49: Distribución de campo eléctrico (izquierda) y magnético (derecha) de la antena MQ en la banda de PCS.

Sin embargo, en la Figura 6.50 se aprecia el nuevo modo creado al introducir la ranura, para cubrir la banda de UMTS. Se puede observar claramente cómo la ranura entra en resonancia para estas frecuencias, en las que la longitud de onda es el doble que la longitud de la ranura.
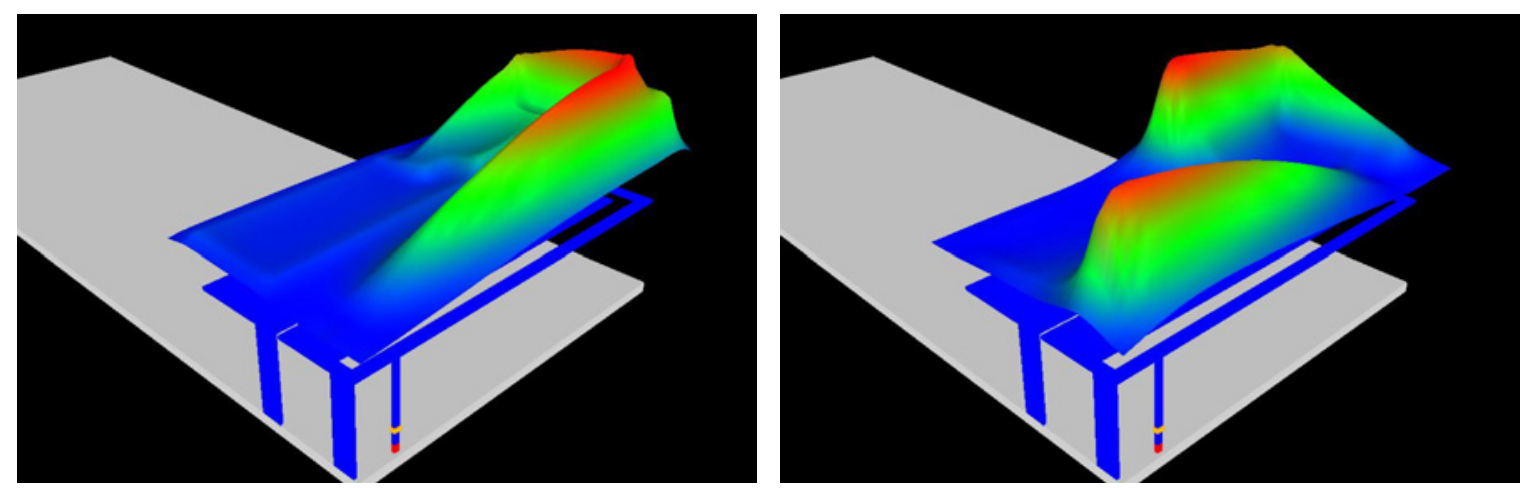

Figura 6.50: Distribución de campo eléctrico (izquierda) y magnético (derecha) de la antena MQ en la banda de UMTS.

Los diagramas de radiación de la antena en las bandas de GSM, DCS y PCS se recogen en la Figura 6.51. Sus características son similares a las de la antena MT descrita en el apartado 6.4.2. Como la introducción de la ranura a lo largo de la rama de GSM no altera significativamente la distribución de campos, tampoco se modifica el diagrama de radiación. 

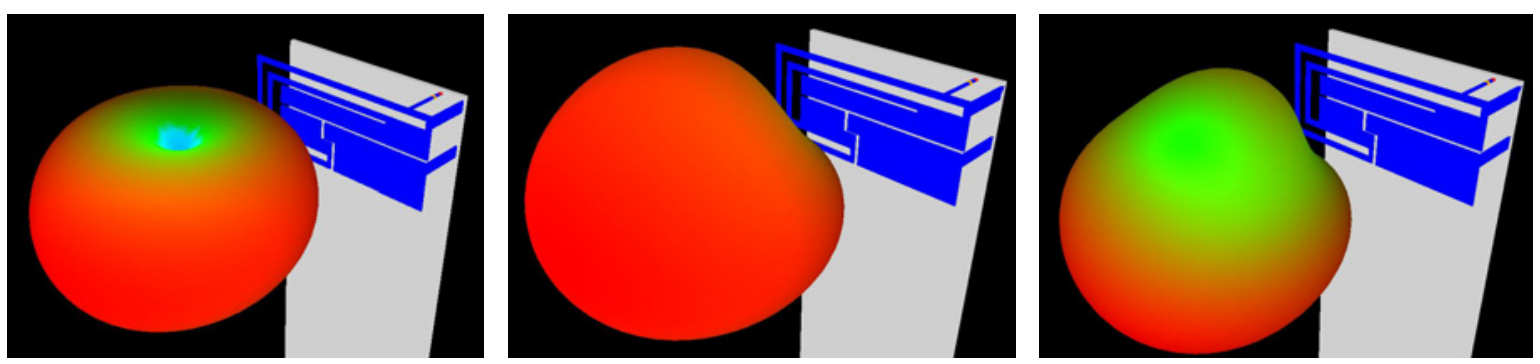

Figura 6.51: Diagrama de radiación de la antena MQ en las bandas de GSM (izquierda), y DCS (centro) y PCS (derecha).

En el caso de la banda de UMTS, el diagrama tiene una forma más irregular, tal y como se aprecia en la Figura 6.52. Esto es debido, por una parte, al hecho de que en este caso el elemento radiante es una ranura, cuyo comportamiento se ve fuertemente afectado por la presencia del plano de masa, y por otra parte la forma en "C" de ésta. Sin embargo esto no es ningún inconveniente importante para su utilización en terminales móviles, puesto que la orientación de estos es aleatoria.

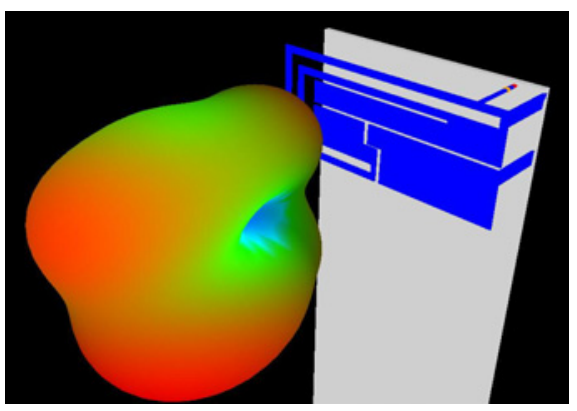

Figura 6.52: Diagrama de radiación de la antena MQ en la banda de UMTS.

\subsection{ConClusión.}

En este capítulo se han descrito antenas de dos, tres o más bandas, que permiten cubrir los principales estándares de comunicaciones móviles. En todos los casos se ha utilizado una combinación de parches activos y parásitos, e incluso una ranura. La principal ventaja de estas estructuras es que los diferentes modos resonantes se obtienen excitando la estructura por un único puerto. Sin embargo, su reducido tamaño y su complejidad pueden requerir la utilización de algún tipo de soporte, cuyo efecto se estudiará en detalle en el siguiente capítulo.

En lo referente a antenas de cuatro bandas, que para incluir por ejemplo los estándares de UMTS o WLAN, se ha visto que se logran resultados interesantes. Sin embargo, en la práctica se preferirá utilizar parches con dos puertos distintos, debido al alto costo de los duplexores necesarios para cubrir un rango de frecuencias tan elevado. Esto originará otros problemas, ligados al aíslamiento entre los diferentes puertos. 


\subsection{Biblografía.}

[6.1] Dou, W.P. y Chia, Y.W.M., 'Novel Meandered Planar Inverted-F Antena for TripleFrequency Operatione', Microwave and Optical Tech. Lett., 2000, vol. 27, n 1, pp. 58-60.

[6.2] Hsiao, F.R., Chen, H.T., Chiou, T.W., Lee, G.Y. y Wong, K.L., 'A Dual-Band Planar Inverted-F Patch Antenna with a Branch-Line Slit', Microwave and Optical Tech. Lett., 2002, vol. 32, n 4, pp. 310-312.

[6.3] Salonen, P., Keskilammi, M. y Kivikoski, M., 'New Slot Configurations for Dual-Band Planar Inverted-F Antenna', Microwave and Optical Tech. Lett., 2001, vol. 28, n 5, pp. 293-298.

[6.4] Sánchez Hernández D. y Martínez Vázquez, M., ‘Antena Dual para Terminales Móviles', n’ de solicitud: P-200002704, Oficina Española de patentes y Marcas.

[6.5] Martínez-Vázquez, M. y Geissler, M., 'Planare Mobilfunkantenne', Patente nacional DE 10054 192, Deutschen Patent- und Markenamt, Alemania, 2002.

[6.6] Virga, K.L. y Rahmat-Samii, Y., 'Low Profile Enhanced Bandwidth PIFA Antennas for Wireless Communications Packaging', IEEE Trans. on Antennas and Propagat., 1997, vol. $45, \mathrm{n}^{\circ} 10$, pp. 1879-1888.

[6.7] Ollikainen, J., Kivekäs, O., Toropainen, A. y Vainikainen, P., 'Internal Dual-Band Patch Antenna for Mobile Phones', Millenium Conf. on Antennas and Propag. AP-2000, 2000.

[6.8] Manteuffel, D., Bahr, A., Heberling, D. y Wolff, I., 'Design Considerations for Integrated Mobile Phone Antennas', IEE Int. Conf. on Antennas and Propag., 2001.

[6.9] Geissler, M. y Martínez-Vázquez, M., 'Mehrbandantenne mit parasitären Strahlern', n de solicitud 10204 079.6, Deutschen Patent- und Markenamt, Alemania, 2002 (concesión prevista en Agosto 2003).

[6.10] Martínez-Vázquez M. y Litschke O., 'Novel Triple-Band Antennas for Personal Communicatons Handsets', IEEE Antennas and Propagat. Society. Symp., 2002.

[6.11] Martínez-Vázquez M. y Litschke O., 'Design Considerations for Quad-Band Antennas Integrated in Personal Communications Devices', Journées Internationales de Nice sur les Antennes, 2002.

[6.12] Wien, A., 'Simulation Based Design of Integrated Multi-Band Antennas for Mobile Communication', Antenna Systems \& Technology, 2002, vol. 5, $\mathrm{n}^{\circ} 6$. 


\section{Capítulo 7: INFLUENCIA DE LOS MATERIALES.}

Nunca existe error tan grande como el de no proseguir.

Jex Blake

\subsection{INTRODUCCIÓN.}

En el capítulo anterior se describían diferentes antenas multibanda, y sus principales características de funcionamiento. En este capítulo se pretende determinar el efecto de su integración en un terminal, desde el punto de vista de los materiales utilizados en la fabricación del aparato.

Así, en la Figura 7.1 se recoge la configuración típica del problema para un parche genérico integrado en un terminal de comunicaciones móviles. Para garantizar la estabilidad y robustez de la estructura, el parche radiante suele ir sujeto, o incluso impreso, sobre un soporte de material dieléctrico, normalmente un material plástico de bajo coste y características dieléctricas desconocidas.

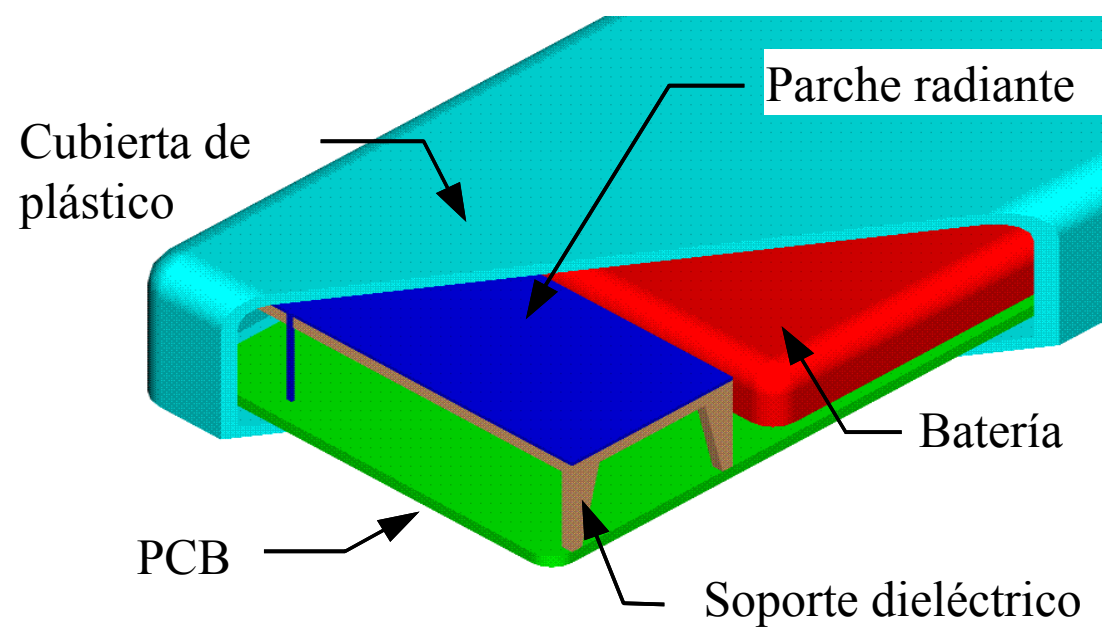

Figura 7.1: Antena parche genérica integrada en un terminal.

Del mismo modo, sobre el parche se coloca la carcasa del terminal, también de plástico normalmente. Los efectos de estos materiales se valorarán en función de los cambios en el comportamiento de la antena, en lo que atañe a las pérdidas de retorno y a su eficiencia. 


\subsection{MEDIDA DE LA EFICIENCIA DE LA ANTENA.}

\subsubsection{Eficiencia.}

La eficiencia es una magnitud que suscita cada vez más interés a la hora de analizar el comportamiento de una antena. En efecto, en el caso de terminales de comunicaciones móviles, cuya orientación espacial es totalmente aleatoria y es importante optimizar el uso de la potencia entregada a la antena, los diagramas de radiación no bastan para dar una idea de la calidad del sistema.

Tal y como se indicaba en el Capítulo 3, la eficiencia permite determinar la relación entre la potencia entregada a la antena y la que es realmente radiada por ésta. La eficiencia total se utiliza pues para incluir las pérdidas que se producen en los puertos de entrada y en la estructura misma de la antena [7.1]. Estas pérdidas son debidas a dos factores:

- la desadaptación entre la línea de transmisión y la antena,

๑ las pérdidas óhmicas en los conductores y los dieléctricos.

Así, la eficiencia total $\eta_{0}$ se puede escribir como el producto de la eficiencia de adaptación $\eta_{\mathrm{a}}$, la eficiencia de conducción $\eta_{c}$ y la eficiencia dieléctrica $\eta_{d}$ :

$$
\eta_{0}=\eta_{\mathrm{a}} \eta_{\mathrm{c}} \eta_{\mathrm{d}}
$$

La eficiencia de adaptación se puede determinar analíticamente a partir del coeficiente de reflexión $\Gamma$ a la entrada de la antena como:

$$
\begin{gathered}
\eta_{\mathrm{a}}=1-|\Gamma|^{2} \\
\Gamma=\frac{Z_{\text {in }}-Z_{0}}{Z_{\text {in }}+Z_{0}}
\end{gathered}
$$

Donde $Z_{\text {in }}$ y $Z_{0}$ denotan respectivamente la impedancia de entrada de la antena y la impedancia característica de la línea de transmisión.

La eficiencia de conducción $\eta_{\mathrm{c}} \mathrm{y}$ la eficiencia dieléctrica $\eta_{\mathrm{d}}$, por el contrario, son difíciles de calcular, pero pueden ser determinadas experimentalmente. Sin embargo, como no pueden medirse por separado se las suele agrupar bajo el nombre de eficiencia de radiación, $\eta_{\mathrm{r}}$, cuya expresión se reduce a:

$$
\eta_{\mathrm{r}}=\eta_{\mathrm{c}} \eta_{\mathrm{d}}
$$

Con lo cual la ecuación (7.1) se puede rescribir como:

$$
\eta_{0}=\eta_{\mathrm{a}} \eta_{\mathrm{r}}
$$


Si se considera el circuito equivalente serie de la antena (equivalente de Thévenin), mostrado en la Figura 7.2, la impedancia de entrada de la antena, $Z_{i n}$, se puede escribir:

$$
\begin{gathered}
\mathrm{Z}_{\text {in }}=\mathrm{R}_{\mathrm{A}}+\mathrm{j} \mathrm{X}_{\mathrm{A}} \\
\mathrm{R}_{\mathrm{A}}=\mathrm{R}_{\mathrm{r}}+\mathrm{R}_{\mathrm{L}}
\end{gathered}
$$

Donde $R_{A}$ denota la resistencia de la antena, $X_{A}$ la reactancia, $R_{r}$ la resistencia de radiación y $R_{L}$ la resistencia de pérdidas.

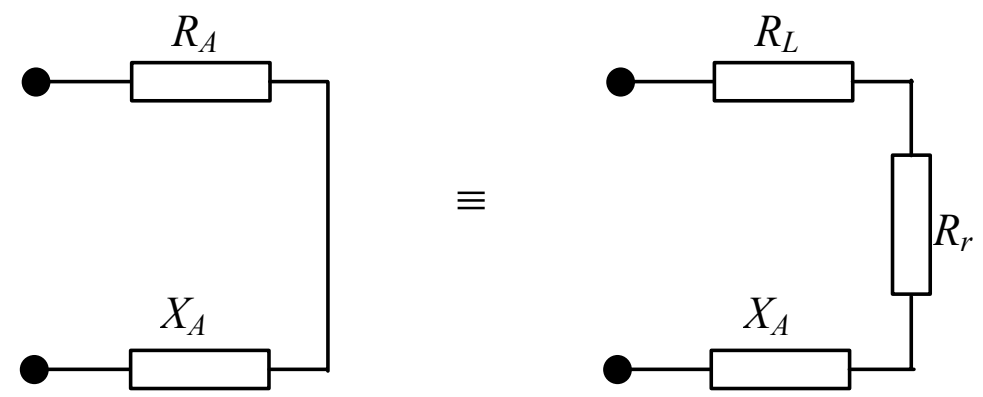

Figura 7.2: Circuito equivalente serie (Thévenin) de una antena.

La eficiencia de radiación, definida como el cociente entre la potencia entregada a la resistencia de radiación $\mathrm{R}_{\mathrm{r}} \mathrm{y}$ la entregada a la resistencia de la antena $\mathrm{R}_{\mathrm{A}}$ se reduce entonces a [7.1]:

$$
\eta_{\mathrm{r}}=\frac{\mathrm{R}_{\mathrm{r}}}{\mathrm{R}_{\mathrm{A}}}=\frac{\mathrm{R}_{\mathrm{r}}}{\mathrm{R}_{\mathrm{L}}+\mathrm{R}_{\mathrm{r}}}
$$

\subsubsection{Procedimiento de medida.}

Para la medida de la eficiencia de las antenas se recurrió a una versión mejorada del método de Wheeler-cap [7.2]-[7.3]. En este caso se utiliza un método de evaluación adaptado a la medida de antenas eléctricamente pequeñas, integradas en terminales de comunicaciones móviles [7.4]. Con este método se puede obtener la eficiencia de la antena a partir del coeficiente de reflexión a la entrada de la antena, $\Gamma$, también denotado $\mathrm{s}_{11}$.

Para ello se procede a medir las pérdidas de la antena en espacio libre, y dentro de una cavidad (Wheeler-cap). Si se considera que la antena sólo tiene pérdidas en serie, como en el equivalente de Thévenin, los circuitos equivalentes son entonces los presentados en la Figura 7.3 y la Figura 7.4.

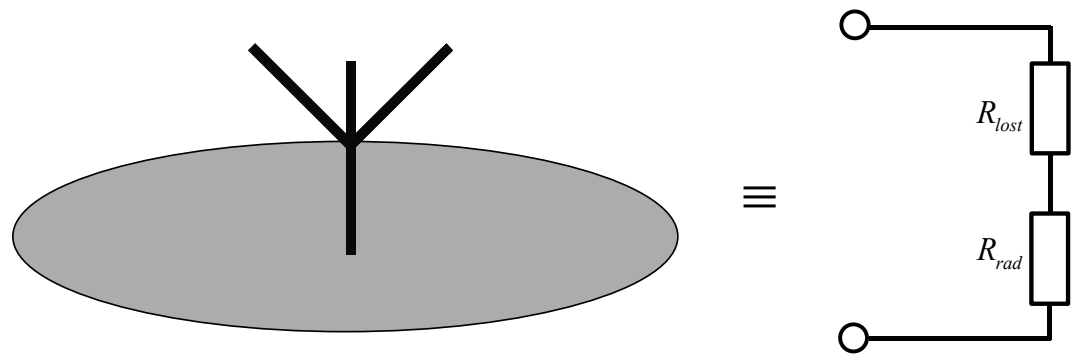

Figura 7.3: Circuito equivalente de las pérdidas de la antena. 


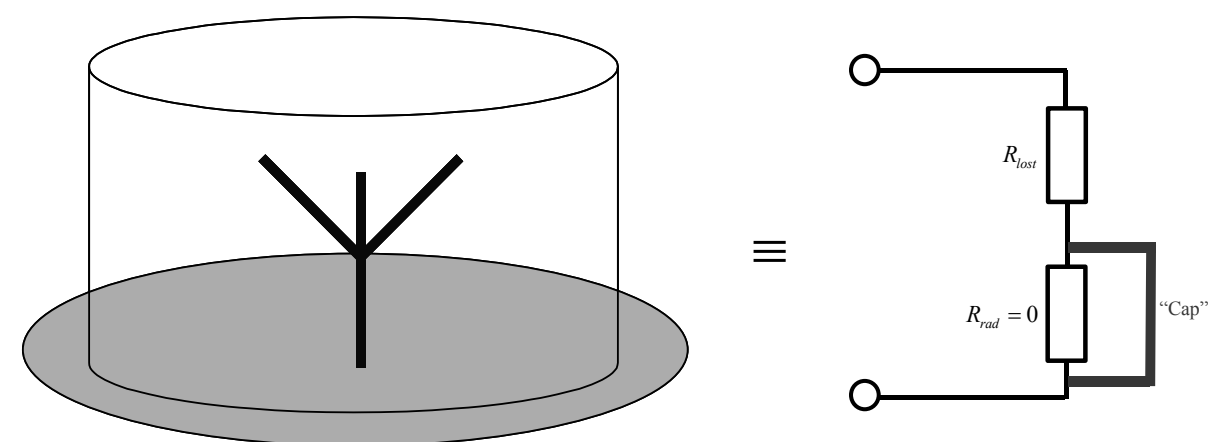

Figura 7.4: Circuito equivalente de las pérdidas de la antena en la cavidad.

La eficiencia de radiación se obtiene como:

$$
\eta_{\mathrm{r}}=\frac{\left|\mathbf{s}_{21}\right|^{2}}{1-\left|\mathbf{s}_{11}\right|^{2}}
$$

El coeficiente de transmisión $\mathrm{s}_{21}$ se determina indirectamente a partir del de reflexión $\mathrm{s}_{11}$ medido para la antena en la cavidad, tal y como se muestra en la Figura 7.5. Se obtienen así las siguientes expresiones:

$$
\begin{gathered}
\left|\mathrm{s}_{21}\right|^{2}=\frac{2}{\frac{1}{\Delta \mathrm{s}_{\max }}+\frac{1}{\Delta \mathrm{s}_{\min }}} \\
\eta_{\mathrm{r}}=\frac{2}{\frac{1}{\Delta \mathrm{s}_{\max }}+\frac{1}{\Delta \mathrm{s}_{\min }}} \cdot \frac{1}{1-\left|\mathrm{s}_{11}\right|^{2}}
\end{gathered}
$$

Los valores de Dsmax y Dsmin se obtienen de la representación gráfica de las medidas en espacio libre y en la cavidad, tal y como se muestra en la carta de Smith de la Figura 7.5.

La relación entre la eficiencia total y la eficiencia de radiación, descrita en la ecuación (7.5), se puede reformular como:

$$
\eta_{0}=\eta_{\mathrm{r}}\left(1-\left|\mathrm{s}_{11}\right|^{2}\right)
$$

Para realizar estas medidas se construyó la cavidad de la Figura 7.6. Se trata de una cavidad cilíndrica cuyo interior está revestido de plata, para optimizar la calidad de las medidas. La cavidad dispone de un cortocircuito deslizante que permite ajustar su tamaño para lograr resultados fiables para frecuencias entre $0,7 \mathrm{GHz}$ y 2,5 GHz. Este rango de frecuencias permite cubrir las principales bandas definidas por los estándares de comunicaciones móviles. 


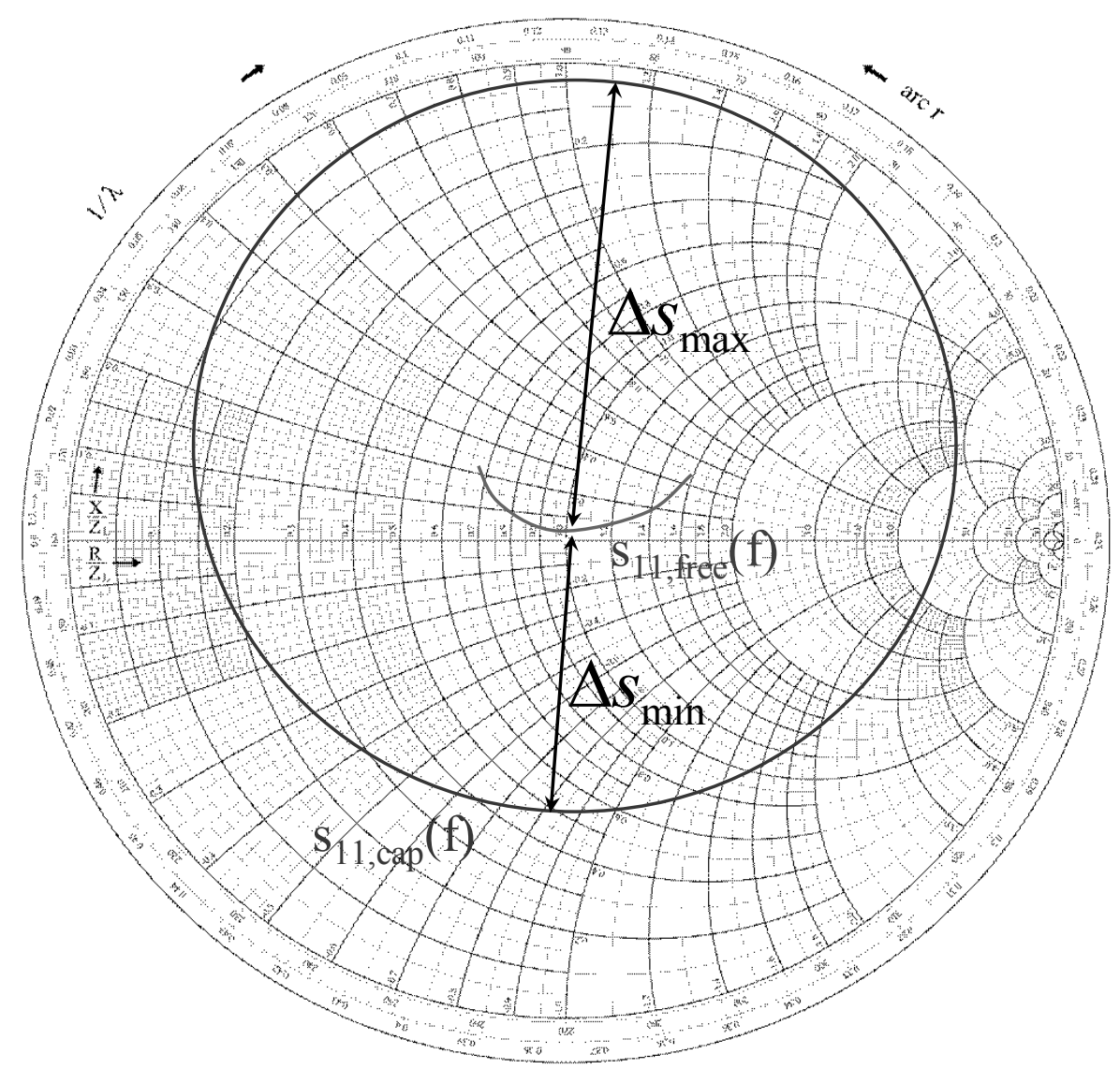

Figura 7.5: Determinación de los parámetros $\Delta \mathrm{s}_{\max } \mathrm{y} \Delta \mathrm{s}_{\min }$ a partir de las medidas.
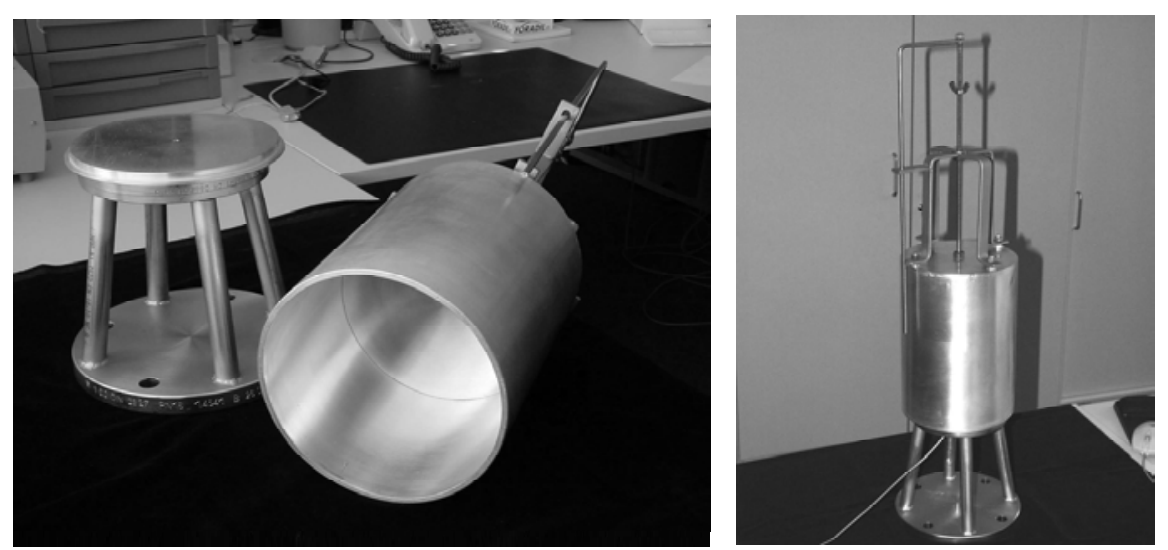

Figura 7.6: Cavidad con cortocircuito deslizante utilizada en las medidas.

Este método permite obtener resultados fiables en cuanto a la eficiencia de la antena en un estado muy temprano del desarrollo, ya que las medidas se realizan de forma pasiva, con la estructura conectada a un analizador de redes mediante un cable. Sin embargo, no es apropiado para las medidas activas. En ese caso habría que medir las ganancias a partir de los diagramas de radiación tridimensionales, o utilizar otros métodos de medida de eficiencia. Entre estos últimos caben destacar el uso de pequeñas cámaras de reverberación, propuesto por Kildal [7.5], y el método de rotación aleatoria desarrollado en la École Polytechnique Fedérale de Lausane [7.6]. 


\subsection{EFECTO DE LA CUBIERTA DE PLÁSTICO.}

En condiciones normales de funcionamiento, las antenas integradas en terminales de comunicaciones móviles están contenidas, como todo el resto de la circuitería, dentro de una cubierta de plástico. Aunque ésta se diseña teniendo en cuenta sobre todo criterios estéticos, su influencia sobre el comportamiento de la antena es importante, pues introduce ciertas variables que hay que tener en cuenta durante la fase de diseño. La cubierta de la antena actuará, por una parte, como un radomo, protegiendo la antena frente a golpes o agresiones externas. Por otra parte, la presencia de este dieléctrico cerca del parche radiante introducirá cambios en las características de adaptación y de radiación de la antena.

Para simular el efecto de la cubierta de plástico del terminal, y así estudiar su efecto, se consideró una lámina de plástico $1 \mathrm{~mm}$ de espesor, y de permitividad $\varepsilon_{\mathrm{r}}=2,9$, situada a $0,5 \mathrm{~mm}$ de la superficie de la antena, tal y como se muestra en la Figura 7.7.

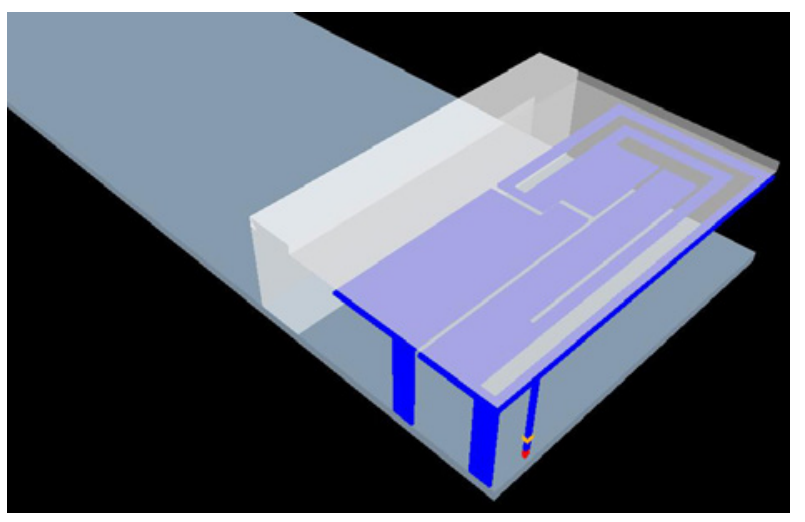

Figura 7.7: Modelo de la antena MQ con cubierta de plástico.

\subsubsection{Pérdidas de retorno.}

En primer lugar se pretende determinar el efecto de la presencia de la antena sobre las características de adaptación de la antena. Las medidas de las pérdidas de retorno de la antena, recogidas en la Figura 7.8., nos muestran tres efectos:

\$ Desviación de las frecuencias de resonancia.

$\infty$ Cambio en el acoplo entre modos.

$\infty$ Disminución de la adaptación.

Al situar la cubierta de plástico sobre la antena cambia la permitividad relativa del espacio situado sobre el parche. Las frecuencias de resonancia que se obtienen son, por lo tanto, menores que cuando no se considera la cubierta. 


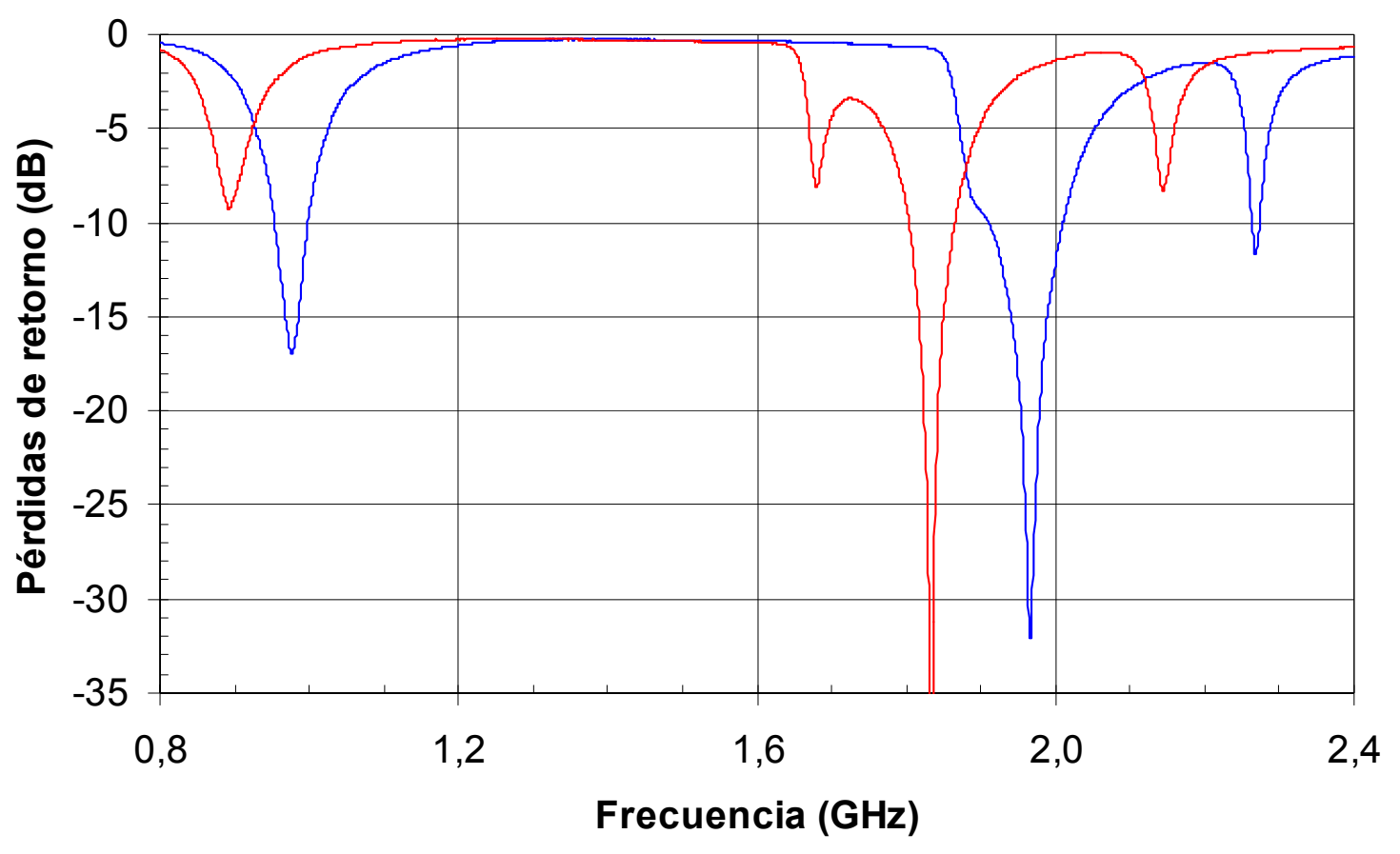

— Antena sólo — Antena con cubierta

Figura 7.8: Pérdidas de retorno medidas de la antena sólo y con cubierta de plástico.

Este efecto se aprecia en todas las bandas de frecuencias consideradas, y se debe tener en cuenta durante la fase de diseño. Así, se diseña normalmente el parche para unas frecuencias ligeramente superiores a las definidas por los estándares, contando con el desplazamiento de las frecuencias de resonancia una vez integrada la antena en el terminal. Este escalado de la antena se hace normalmente de forma empírica, y la exactitud de los resultadoes dependerá en gran medida de la experiencia del diseñador.

La presencia de la cubierta de plástico provoca también cambios en la distribución de campos en las proximidades del parche. Los diferentes acoplamientos se verán, pues, afectados. En este caso, las gráficas de la Figura 7.8 muestran cómo al colocar la cubierta aumenta la separación entre los modos generados para cubrir las bandas de DCS y PCS.

Finalmente, se aprecia también que en el caso de la antena con cubierta de plástico se degradan los niveles de adaptación. Esto es debido a las pérdidas dieléctricas. Este efecto se traduce igualmente en una pérdida de eficiencia, que se discutirá en el apartado siguiente.

\subsubsection{Eficiencia.}

El uso de la cubierta de plástico afectará también a las propiedades de radiación de la antena, al introducir pérdidas dieléctricas. En la Figura 7.9 y la Figura 7.10 se ilustra la diferencia entre los resultados obtenidos en cuanto a eficiencia y pérdidas de retorno con y sin cubierta dieléctrica. También aquí se ha hecho la distinción entre eficiencia de radiación, determinada únicamente por las propiedades del parche, y eficiencia total, que incluye el efecto de la desadaptación. Como se 
puede ver, en los puntos en los que hay una buena adaptación, ambas curvas llegan a coincidir. En el caso ideal de una antena perfectamente adaptada en toda la banda, las dos curvas de eficiencia serían idénticas.

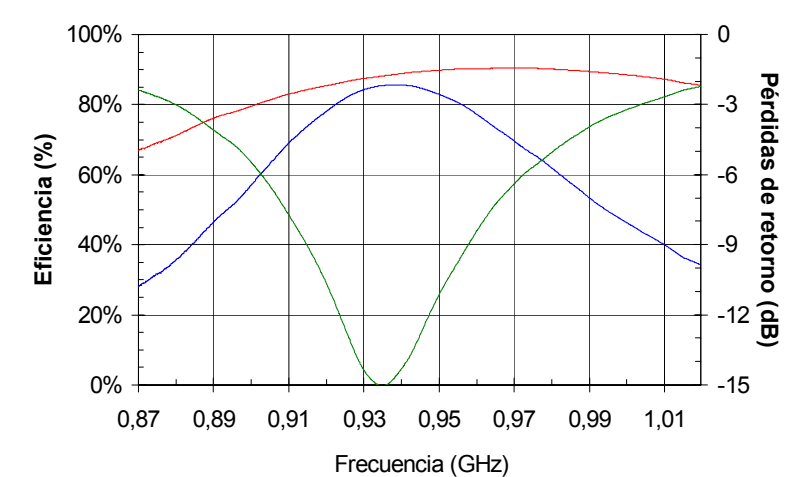

- Eficiencia Total - Eficiencia de radiation

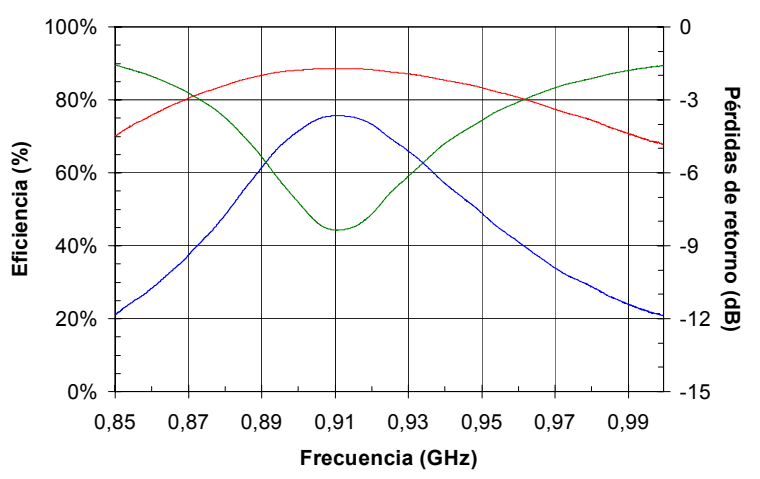

— Pérdidas de retorno

Figura 7.9: Eficiencia de radiación, eficiencia total y pérdidas de retorno de la antena sin cubierta de plástico (izquierda) y con cubierta (derecha) para la banda de GSM.
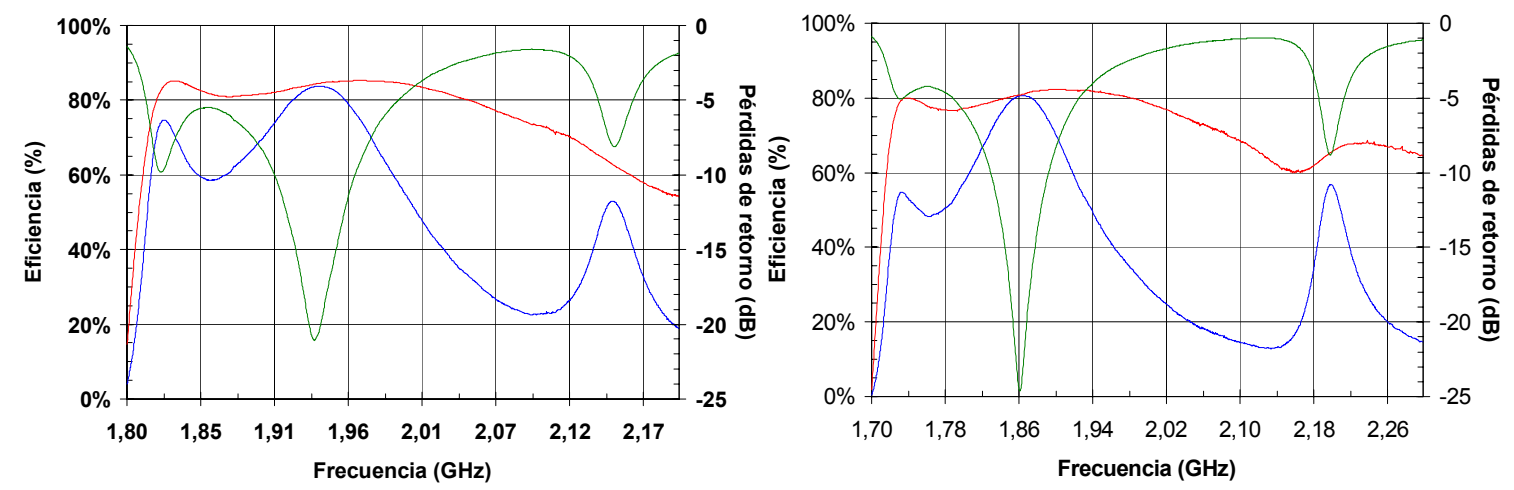

- Eficiencia Total _ Eficiencia de radiation

- Pérdidas de retorno

Figura 7.10: Eficiencia de radiación, eficiencia total y pérdidas de retorno de la antena sin cubierta de plástico (izquierda) y con cubierta (derecha) para las bandas de DCS, PCS y UMTS.

De las curvas de la Figura 7.9 y la Figura 7.10 se deduce que las restricciones de ancho de banda, en lo referente a la eficiencia no están determinadas por la antena es sí. En efecto, la eficiencia de radiación es alta, y su comportamiento se mantiene relativamente constante en las bandas de frecuencias de interés, tanto si se incluye el efecto de la cubierta como si no. Como esta pérdida de eficiencia se debe a la desadaptación, sería posible compensarla mediante el uso de una red de adaptación pasiva compuesta de elementos concentrados. Una técnica similar ha sido ya utilizada, por ejemplo, para mejorar el el funcionamiento a frecuencias de GSM de una PIFA de doble banda [7.7]. Una red de adaptación con elementos distribuidos permitía doblar el acho de banda de GSM y su eficiencia, aunque se perdía ancho de banda en las frecuencias de DCS.

En el caso de la antena con cubierta de plástico, se observa una pérdida de eficiencia total de alrededor del 5\% con respecto a la antena sin cubierta. Esta disminución es prácticamente constante con la frecuencia, y se debe atribuir a las pérdidas en los dieléctricos. No puede ser compensada pues con el uso de una red de adaptación.

En todo caso, los valores de eficiencia total obtenidos sin utilizar ningún circuito de adaptación, 
con una eficiencia total de más del $50 \%$ en todas las bandas, son lo suficientemente buenos como para avalar el uso de esta antena en terminales móviles.

\subsection{EFECTO DEL SOPORTE DIELÉCTRICO Y DE LA METALIZACIÓN.}

Otro aspecto que se debe tener en cuenta es el uso de un soporte dieléctrico que proporciona estabilidad mecánica a la estructura. En efecto, al aumentar el número de bandas de frecuencias que se quiere cubrir, y reducirse el tamaño de la antena, se tienen a menudo estructuras muy delicadas e inestables. Los elementos radiantes son cada vez más finos, o contienen ranuras, se pueden usar elementos parásitos y acoplamientos, etc. La exactitud en el posicionamiento de las piezas tendrá por lo tanto una gran influencia sobre el funcionamiento de la antena.

El uso de un soporte dieléctrico tendrá un efecto importante sobre los resultados. Por una parte, modificará la permitividad relativa bajo el parche, lo que se traduce en un desplazamiento de las frecuencias de resonancia. También cambiarán las distribuciones de los campos, y acoplamientos entre las diferentes partes de los parches.

Finalmente, la eficiencia también se verá afectada por la utilización de un soporte dieléctrico, puesto que su presencia supone la introducción de nuevas pérdidas que reducirán la eficiencia total.

\subsubsection{Adaptación y frecuencias de resonancia.}

Para estudiar el efecto de un soporte de plástico bajo la antena se consideró el parche $\mathrm{M}$ dual presentado en el apartado 6.2.2. Esta antena tenía unas dimensiones de $39 \mathrm{~mm}$ x $26 \mathrm{~mm}$ x $8 \mathrm{~mm}$, y permitía cubrir los estándares de GSM y DCS. Para proporcionar resistencia a la estructura se utiliza un soporte dieléctrico de $1 \mathrm{~mm}$ de espesor. La estructura utilizada en las simulaciones se muestra en la Figura 7.11.

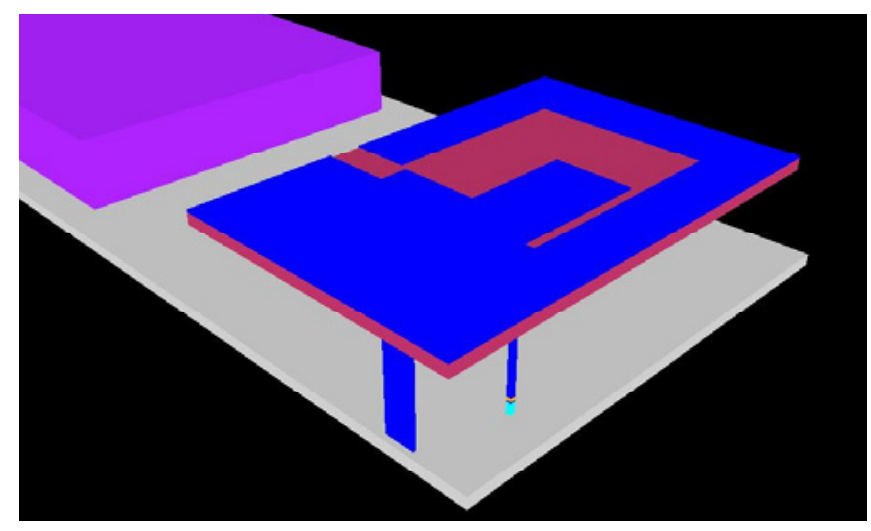

Figura 7.11: Modelo de simulación de la antena M con soporte de plástico.

La elección del material para su realización condicionará los resultados obtenidos. En la Tabla 7.1 
se recogen las características proporcionadas por el fabricante para algunos de los materiales considerados.

Tabla 7.1: Características de los plásticos considerados para la fabricación del soporte.

\begin{tabular}{|c|c|c|}
\hline Material & Tipo & $\boldsymbol{\varepsilon}_{\mathbf{r}}(\mathbf{1 G H z})$ \\
\hline Topas $\AA$ & COC (Cyclic Olefin Copolymer) & $2,25(100 \mathrm{MHz})$ \\
\hline Vectra ${ }^{\circledR}$ A430 & LCP (Liquid Cristal Polymer) & 3,1 \\
\hline Fortron ${ }^{\circledR} 1140$ L4 & PPS (Polyphenylensulphide) & 4,5 \\
\hline
\end{tabular}

Los materiales dieléctricos se han considerado ideales, es decir, sin pérdidas, durante la simulación. Esto es debido a que, al tratarse de materiales no específicos para aplicaciones de alta frecuencia, las pérdidas, dadas por $\tan (\delta)$, no suelen venir especificadas por el fabricante, al menos en las frecuencias consideradas. Para deducirlas es necesario llevar a cabo medidas experimentales de los parámetros dieléctricos [7.8].

En la Figura 7.12 se presentan las pérdidas de retorno simuladas para los materiales descritos en la Tabla 7.1, comparadas con el caso de la antena sin soporte. Se puede observar cómo, al aumentar la permitividad del dieléctrico, la frecuencia de resonancia de cada banda disminuye. Esto es debido al aumento de la permitividad efectiva bajo el parche. Así, al aumentar la permitividad, la antena es más grande, en términos de longitud de onda, lo que se traduce por una disminución de las frecuencias de resonancia.

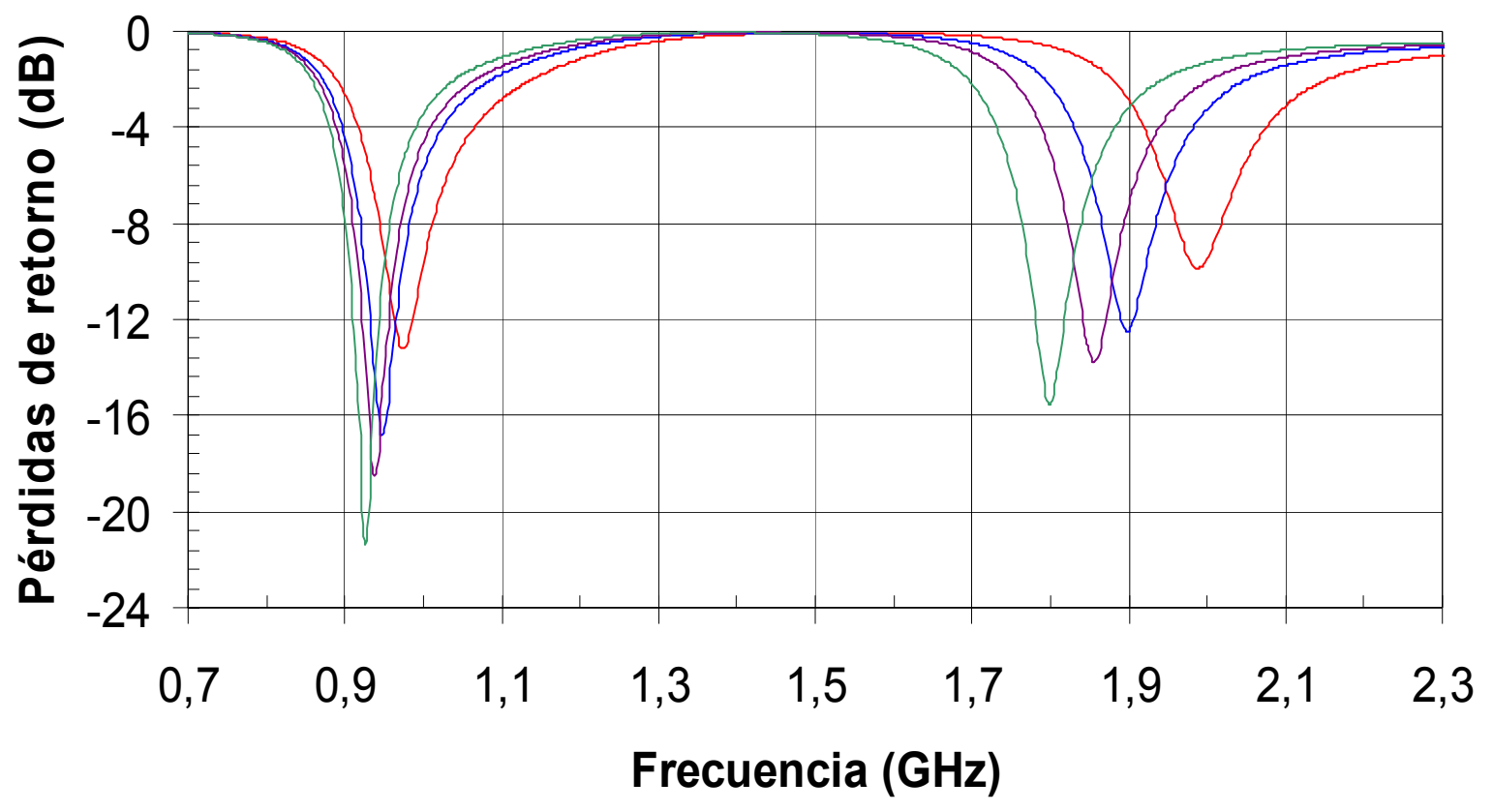

- Aire - Topas (er=2,25) - Vectra $(e r=3,1)$ - Fortron $(e r=4,5)$

Figura 7.12: Pérdidas de retorno simuladas, para soportes de con diferentes materiales plásticos comerciales.

El uso de un material dieléctrico para la fabricación del soporte altera igualmente la distribución de campos en las proximidades de la antena. El efecto capacitivo entre las distintas partes del parche 
aumentará, ya que el campo eléctrico se concentrará más en el material dieléctrico.

En la Figura 7.13 y la Figura 7.14 se puede comparar la distribución de campo eléctrico para el caso de una antena sin soporte y un parche con soporte de Fortron ${ }^{\circledR}$, cuya permitividad relativa es $\varepsilon_{r}=4,5$.

En este caso las diferencias observadas en las distribuciones de campos no son excwsivamente importantes, ya que el espesor del plástico es pequeño en comparación con la altura total del parche y las dimensiones de la antena.
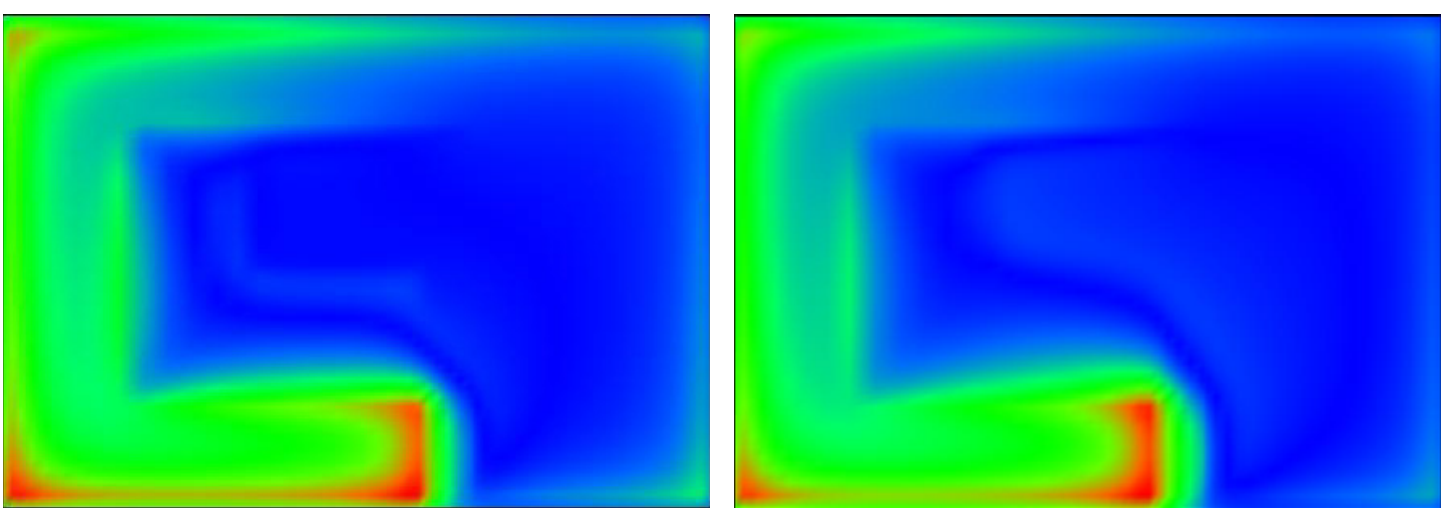

Figura 7.13: Distribución de campo eléctrico en la banda de GSM para la antena sin soporte dieléctrico (izquierda) y con soporte de Fortron ${ }^{\circledR}$ (derecha).
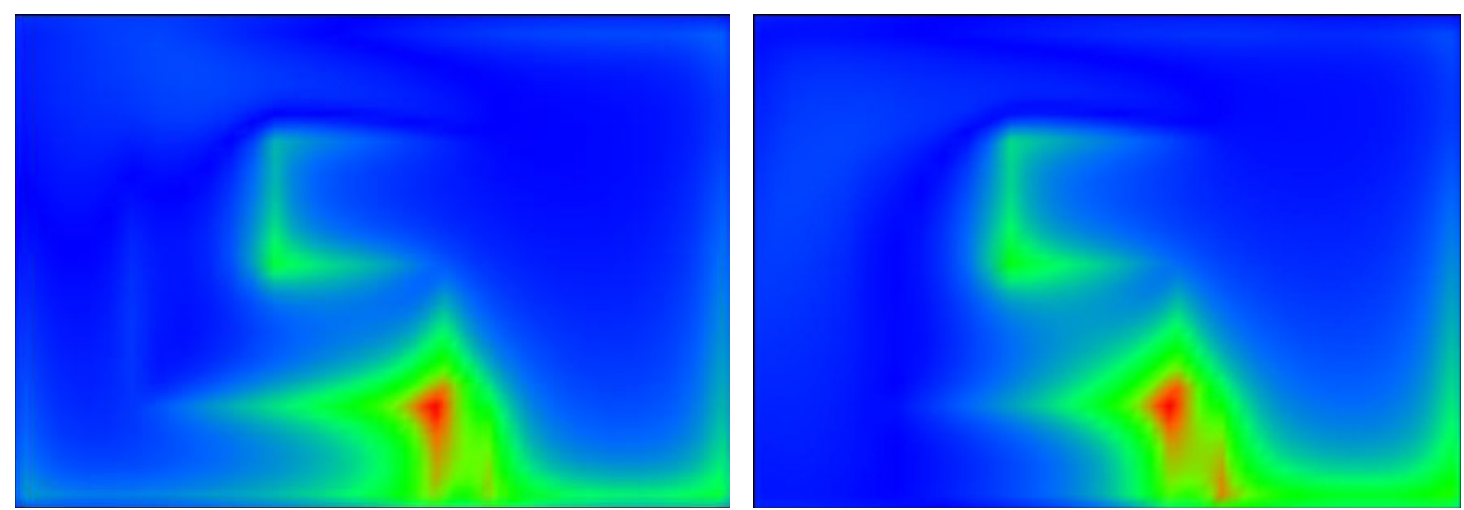

Figura 7.14: Distribución de campo eléctrico en la banda de DCS para la antena sin soporte dieléctrico (izquierda) y con soporte de Fortron ${ }^{\circledR}$ (derecha).

El efecto sería mucho mayor si consideráramos la antena de cuatro bandas MQ descrita en el Capítulo 6, ya que en ella los acoplamientos tienen más influencia sobre el comportamiento de la antena. En este caso se contemplaron dos alternativas: la antena sólo sobre el plano de masa, y una antena impresa sobre un substrato de Duroid de $1 \mathrm{~mm}$ de espesor, y con una constante dieléctrica $\varepsilon_{\mathrm{r}}=2,2[7.9]$.

En la gráfica de la Figura 7.15 se puede observar cómo, para la antena sobre Duroid, la frecuencia de resonancia disminuye, al tiempo que empeora la adaptación. 


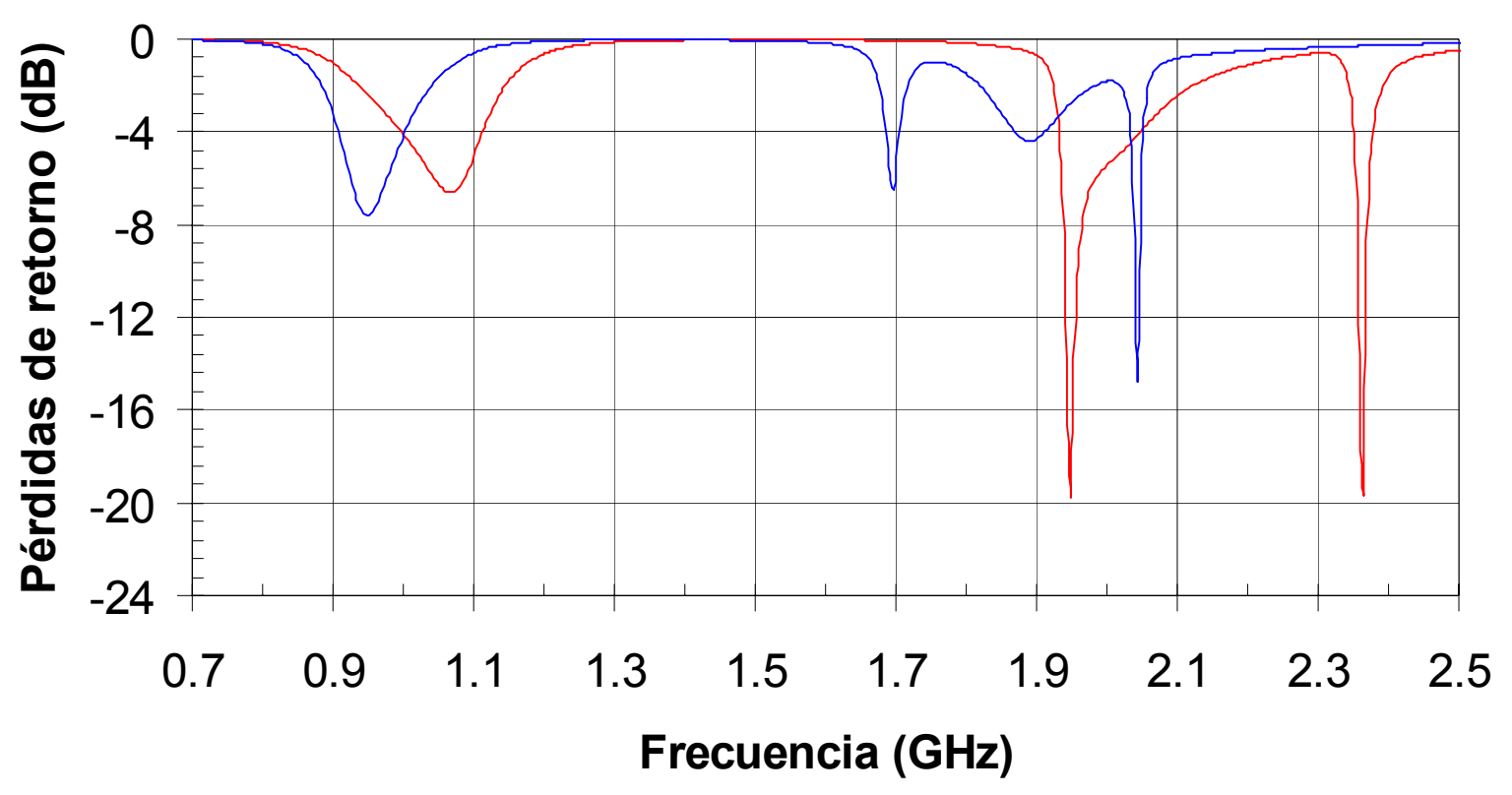

\section{- Aire - Duroid (er=2.2)}

Figura 7.15: Pérdidas de retorno de la antena sin soporte dieléctrico y con soporte de Teflón.

De la Figura 7.16 a la Figura 7.19 se representan las distribucianes de campo eléctrico de la antena MQ para los dos casos considerados. Se aprecia claramente cómo el hecho de utilizar un substrato dieléctrico altera considerablemente las distribuciones de campo y lo acoplamientos entre las diferentes partes de la estructura. El efecto es más evidente conforme se aumenta la frecuencia de trabajo. Así, en la banda de UMTS se puede ver cómo, al usar el dieléctrico, entra en resonancia la ranura, que se mantenía pasiva a esa frecuencia si para la antena sin substrato.
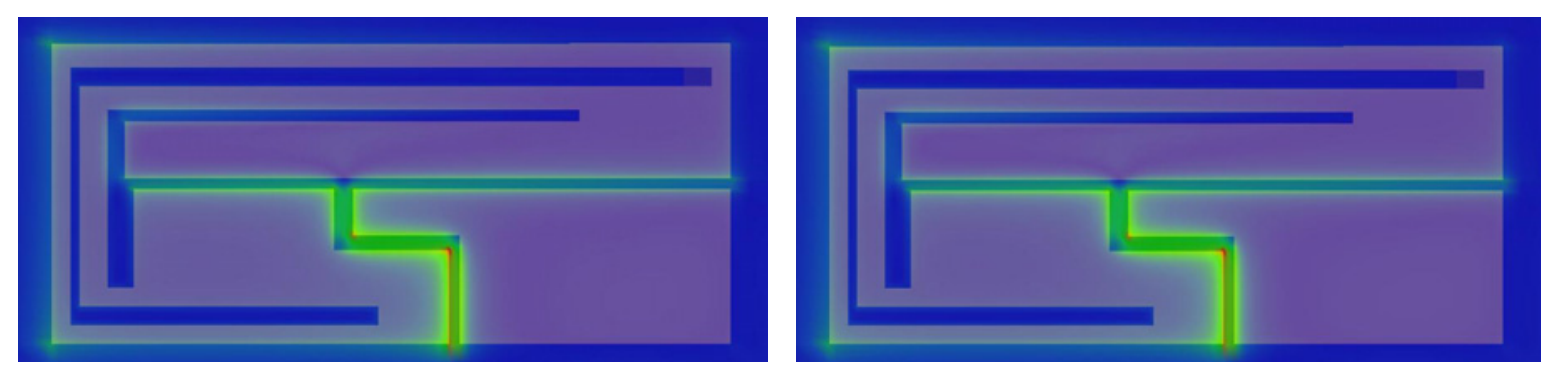

Figura 7.16: Distribución de campo eléctrico en la banda de GSM para la antena sin soporte dieléctrico (izquierda) y con soporte de Teflón (derecha).
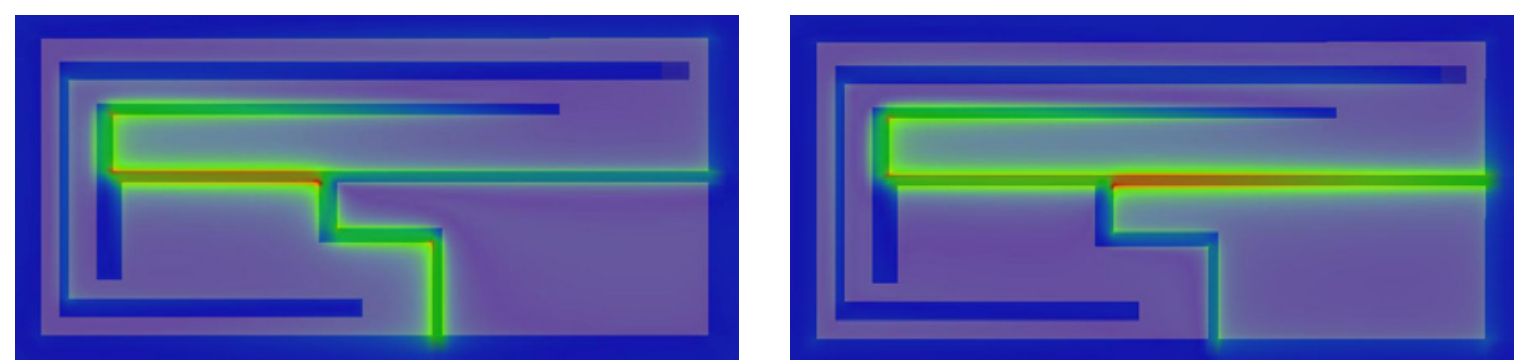

Figura 7.17: Distribución de campo eléctrico en la banda de DCS para la antena sin soporte dieléctrico (izquierda) y con soporte de Teflón (derecha). 

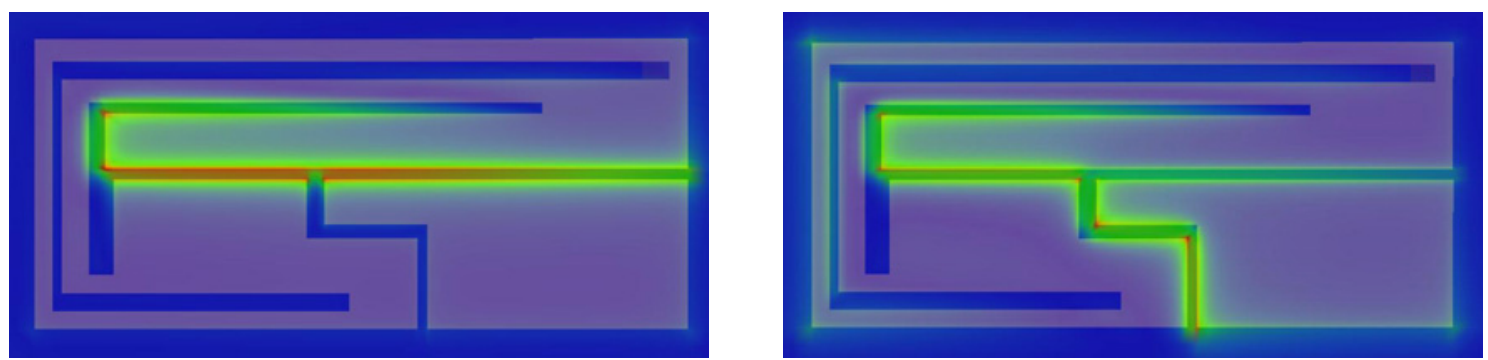

Figura 7.18: Distribución de campo eléctrico en la banda de PCS para la antena sin soporte dieléctrico (izquierda) y con soporte de Teflón (derecha).
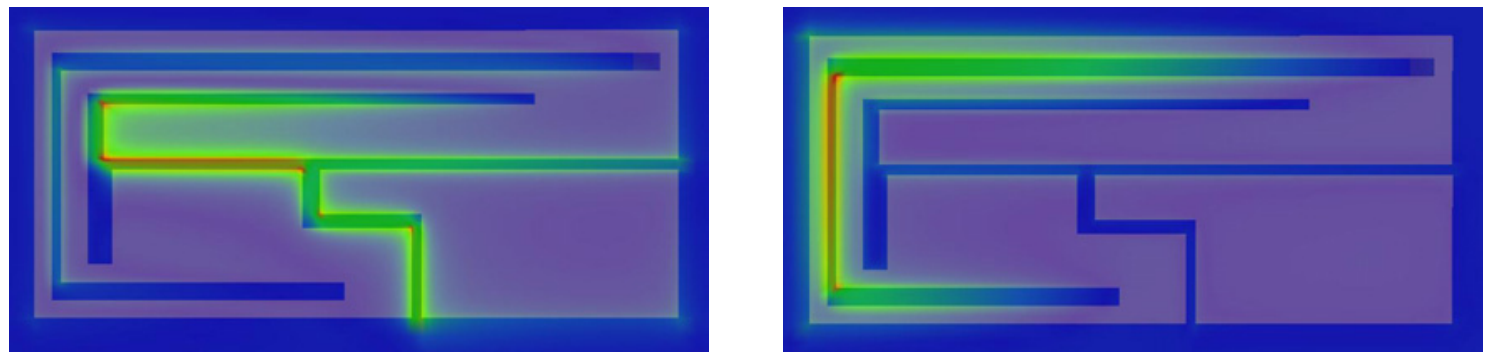

Figura 7.19: Distribución de campo eléctrico en la banda de UMTS para la antena sin soporte dieléctrico (izquierda) y con soporte de Teflón (derecha).

\subsubsection{Eficiencia.}

El uso de un soporte dieléctrico repercutirá también sobre la eficiencia de la antena, al introducir nuevas pérdidas en los dieléctricos. Es por lo tanto importante determinar cuál será el efecto de un determinado material utilizado para la fabricación del soporte. Desgraciadamente, los datos disponibles no son siempre exactos, así que hay que llevar a cabo medidas para determinar la influencia del soporte. En este caso, consideraremos la antena $\mathrm{M}$ de doble banda con conector integrado presentada en el apartado 0 , y veremos cómo se altera su comportamiento, en términos de eficiencia, debido al uso de diferentes materiales. La estructura de la antena se muestra en la Figura 7.20 .

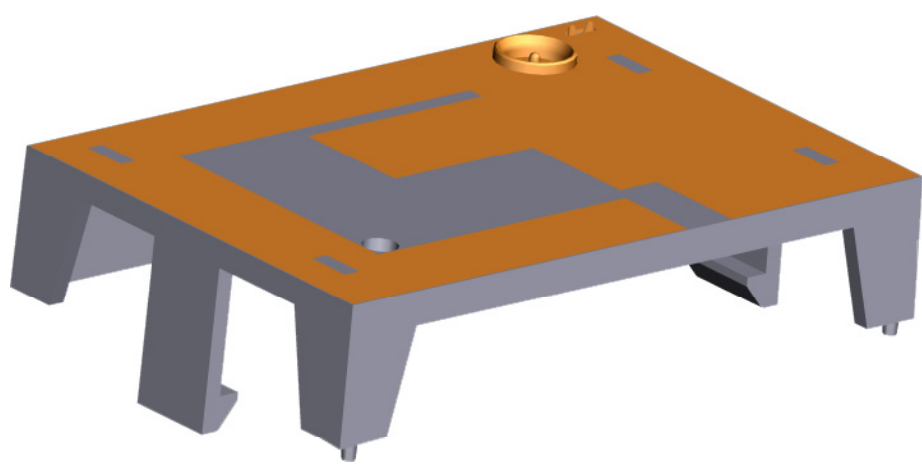

Figura 7.20: Antena M de doble banda con conector integrado.

Consiste en un cuerpo de plástico de $39 \mathrm{~mm}$ x $26 \mathrm{~mm}$ x $8 \mathrm{~mm}$, sobre el que se coloca la estructura metálica radiante. Se estudiarán diferentes combinaciones de metal y plástico, aunque todas tendrán las mismas dimensiones, el mismo conector, y estarán situadas sobre el mismo plano de masa, e 
integradas en el mismo cuerpo de plástico para simular el efecto de la cubierta del terminal.

Las características de los plásticos utilizados para la fabricación del soporte son las descritas anteriormente en la Tabla 7.1.

En primer lugar, se considera un parche de latón, con un soporte de Vectra ${ }^{\circledR}$. En la Figura 7.21 se puede observar que se obtienen unos niveles de eficiencia de radiación muy elevados, entre el $80 \%$ y el $90 \%$. Se puede deducir que esta combinación de materiales se adapta perfectamente a las necesidades de diseño de antenas para terminales. La eficiencia total sin embargo es menor que la eficiencia de radiación, ya que tiene en cuenta el efecto de las pérdidas de retorno. Éstas, tal y como se vio en el apartado anterior, también dependerán en parte del dieléctrico utilizado.

Si en lugar de latón se utiliza acero para la fabricación del parche, los cambios en la eficiencia son apenas apreciables, tal y como se muestra en la Figura 7.22. Desde el punto de vista electromagnético, ambos metales, latón y acero, pueden ser pues empleados sin problemas en la fabricación de las antenas. La elección de uno u otro dependerá pues de otros criterios, como resistencia mecánica, facilidad de fabricación y precio.

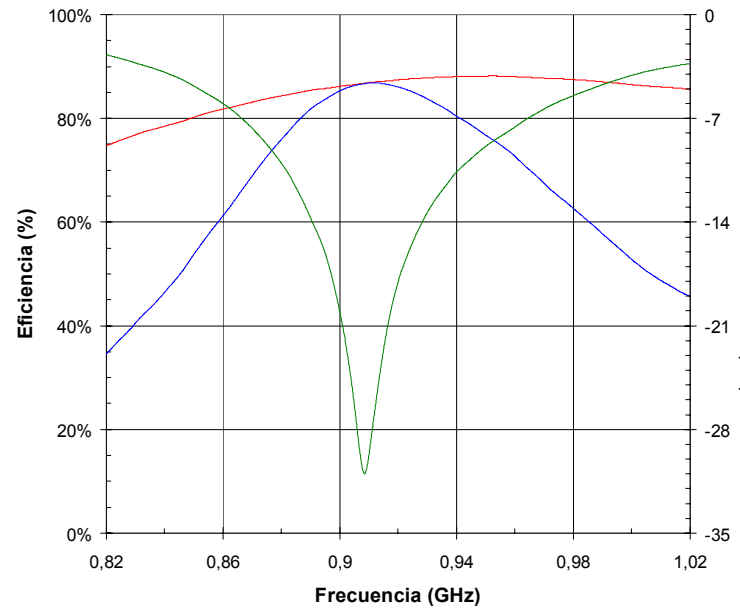

- Eficiencia Total

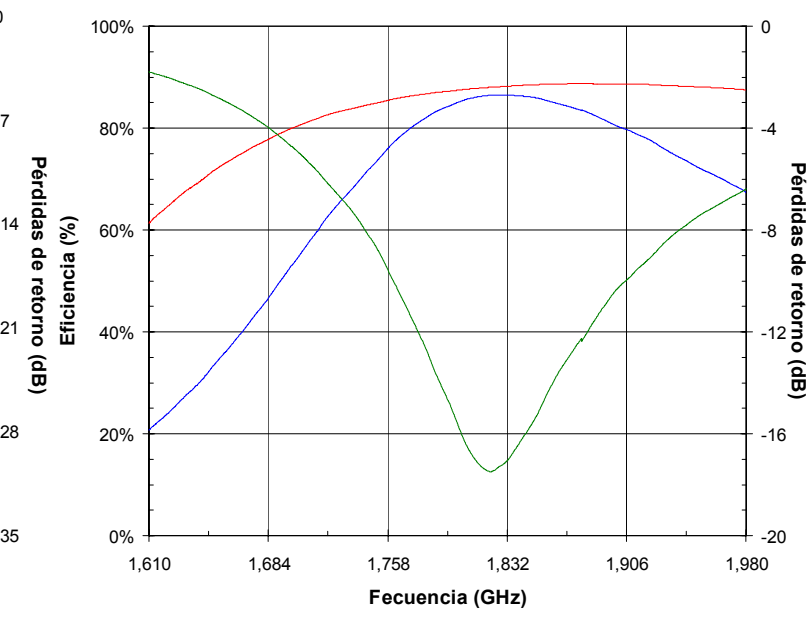

- Pérdidas de retorno

Figura 7.21: Eficiencia de la antena con soporte $\operatorname{Vectra}{ }^{\circledR}$ y parche de latón.

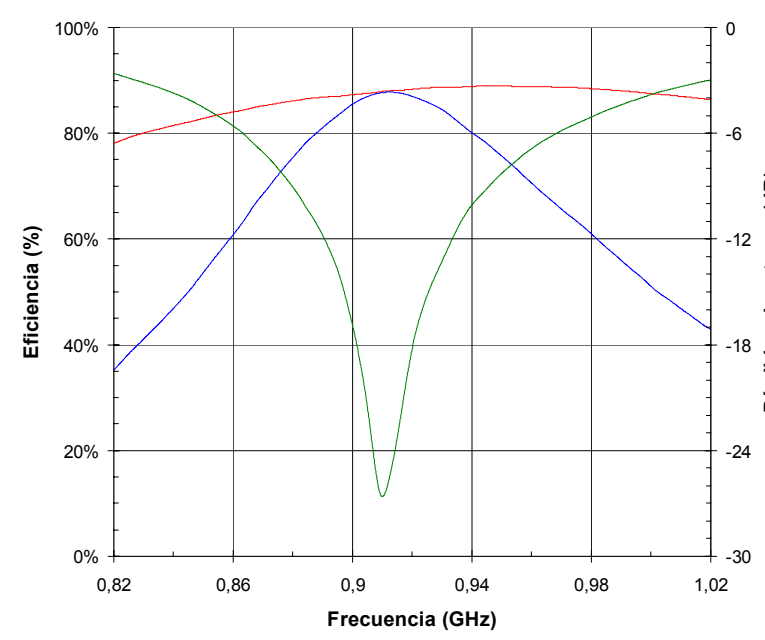

- Eficiencia Total

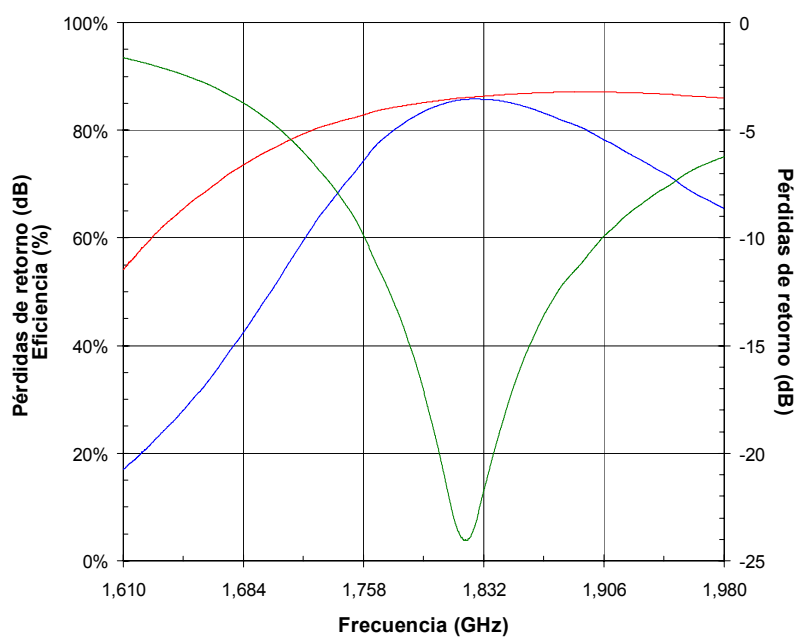

- Pérdidas de retorno

Figura 7.22: Eficiencia de la antena con soporte Vectra ${ }^{\circledR}$ y parche de acero. 
En el caso de un parche de metal con soporte de Topas ${ }^{\circledR}$, la eficiencia de radiación se mantiene similar a la de los casos anteriores, con soporte de Vectra®. Este material plástico puede ser, pues. utilizado igualmente para estas aplicaciones. Sin embargo, en la Figura 7.23 se observa cómo la eficiencia total, aunque elevada, tiene un comportamiento en frecuencia distinto. Esto es debido a la desintonización de la antena, al utilizar un material de permitividad más baja. Sería pues necesario modificar ligeramente el diseño del parche para compensar este desplazamiento en frecuencia.

La última opción que se consideró fue un soporte de Vectra® sobre el que deposita una capa de metal, utilizando tecnología 3D-MID (Metal Injected Devices), tal y como se muestra en la fotografía de la Figura 7.24. Esta tecnología permite realizar estructuras totalmente tridimensionales, pero tiene como desventaja una importante pérdida de eficiencia, debido a la baja calidad de la metalización y a las pérdidas en el dieléctrico.

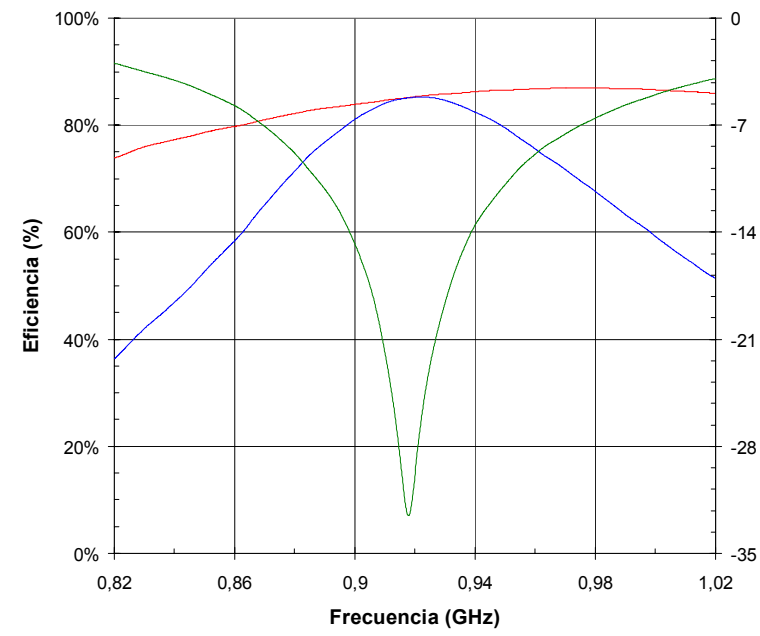

- Eficiencia Total

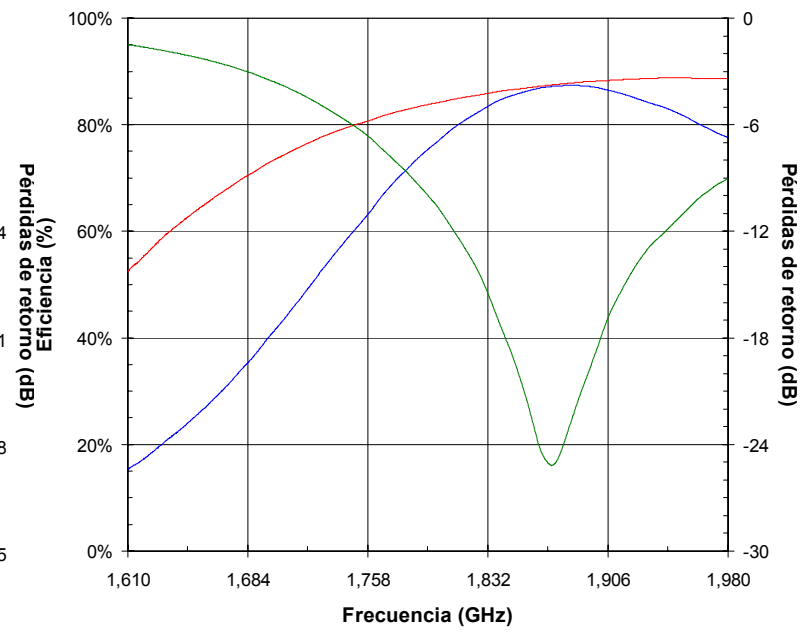

Pérdidas de retorno

Figura 7.23: Eficiencia de la antena con soporte Topas ${ }^{\circledR}$ y parche de acero.

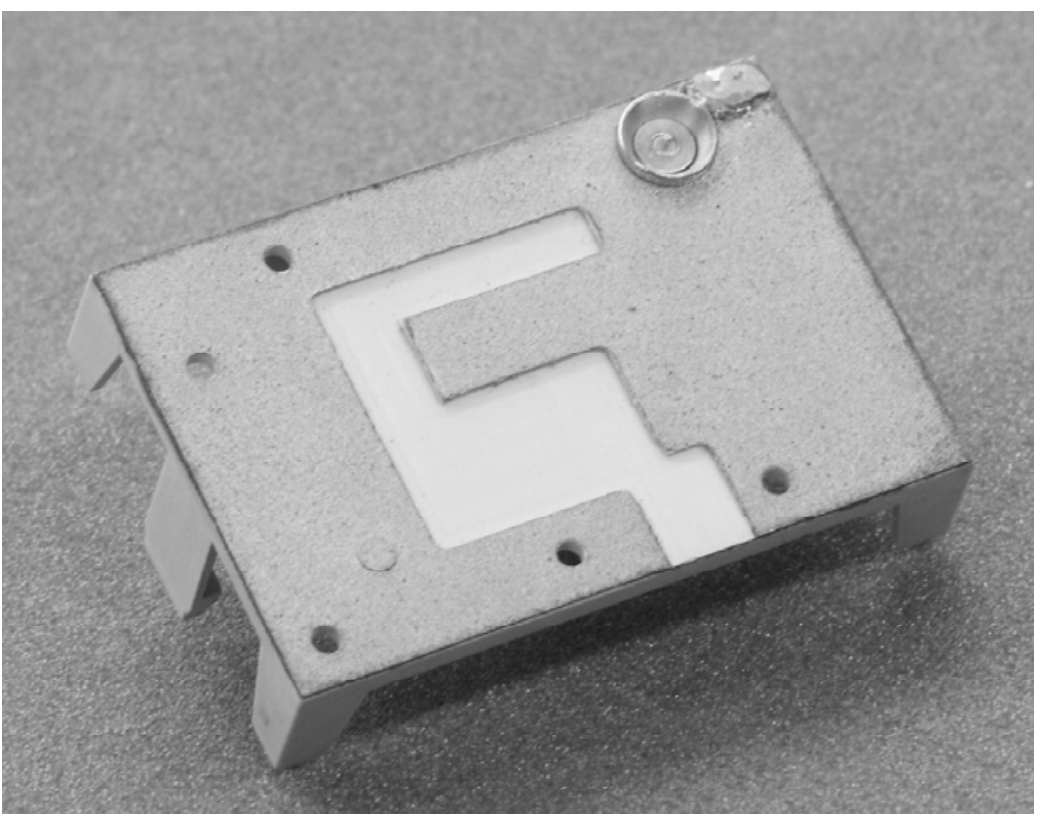

Figura 7.24: Antena M con conector con tecnología 3D-MID. 
En este caso, las pérdidas de los materiales son más importantes que en el caso de las antenas fabricadas por estampación y sujetas por un soporte de plástico. La disminución de la eficiencia de radiación de la antena se aprecia claramente en la Figura 7.25. Ya que estas pérdidas están ligadas a la tecnología y los materiales utilizados, no pueden ser compensadas adecuadamente mediante el uso de redes de adaptación.

A pesar de la pérdida de prestaciones, la eficiencia total se mantiene por encima del $50 \%$ para las bandas de frecuencias de interés. Por lo tanto no hay que descartar el uso de esta tecnología en determinados casos, en los que se requiera mayor flexibilidad en cuanto a la forma de la estructura.

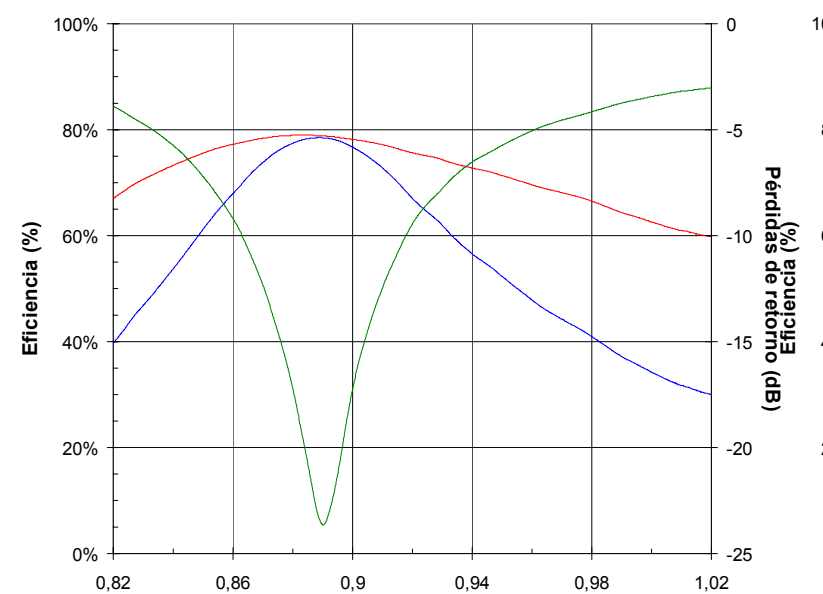

- Eficiencia Total —Eficiencia de radiation

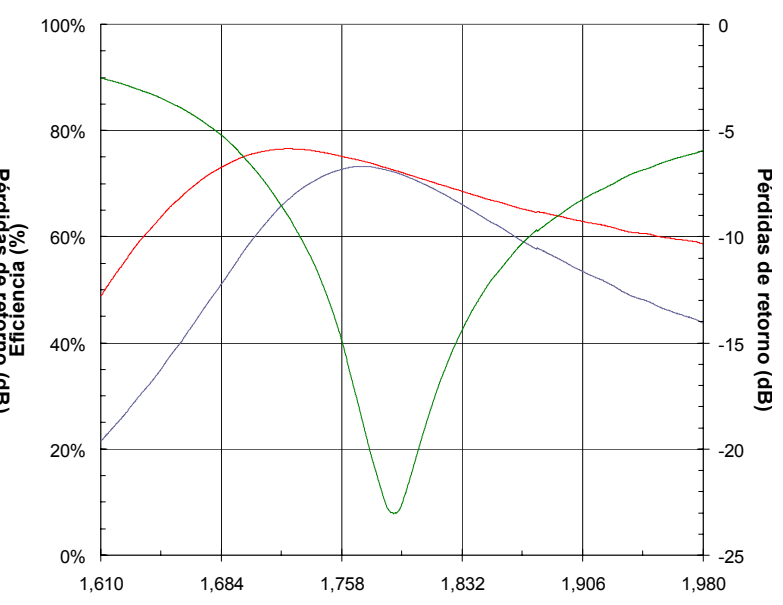

- Pérdidas de retorno

Figura 7.25: Eficiencia de la antena con soporte Vectra® metalizado (MID).

\subsection{CONCLUSIÓN.}

En este capítulo se ha analizado un parámetro importante relacionado con las antenas integradas: su eficiencia. Se ha podido ver cómo los materiales empleados en su fabricación tendrán una influencia significativa sobre el rendimiento total de la estructura. Sin embargo, se ha podido comprobar cómo, para algunos de los conceptos presentados, la eficiencia total obtenida bastaba para cumplir los requisitos establecidos por los fabricantes de terminales. Del mismo modo, las características de adaptación reflejan también el cambio en los materiales utilizados, aunque en este caso el efecto se puede compensar modificando ligeramente la estructura.

\subsection{Biblografía.}

[7.1] Balanis, C.A., 'Antenna Theory, Analysis and Design', $2^{\text {nd }}$ Edition, John Wiley \& Sons, 
1997.

[7.2] Pozar, D.M. y Kaufman, B., 'Comparison of Three Methods for the Measurement of Printed Antenna Efficiency', IEEE Trans. on Antennas and Propagat., 1988, vol. 36, $\mathrm{n}^{\circ}$ 1, pp. 136-139.

[7.3] Johnson, R.H., y McRory, J.G., 'An Improved Small Antenna Radiation-Efficiency Measurement Method', IEEE Antennas and Propagat. Magazine, 1998, vol. 40, n 5, pp. $40-48$.

[7.4] Litschke, O., 'Bestimmung des Wirkungsgrades von Handyantennen mit der Wheeler-CapMethode', Proyecto de Final de Carrera, Gerhard-Mercator-Universität, Duisburg, 2001.

[7.5] Rosengren, K., Kildal, P.S., Carlsson C. y Carlsson, J., 'Characterization of Antennas for Mobile and Wireless Terminals by Using Reverberation Chambers: Improved Accuracy by Platform Stirring', IEEE Antennas and Propagat. Society. Symp., 2001.

[7.6] Skrivervik, A.K., Zurcher, J.-F., Staub, O. y Mosig, J.R., 'PCS antenna design: the challenge of miniaturization', IEEE Antennas and Propagat. Magazine., 2001, vol. 43, $\mathrm{n}^{\circ}$ 4, pp. $12-27$.

[7.7] Ollikainen, J., Kivekäs, O., Icheln, C. Y Vainikainen, P., 'Internal Multiband Antenas Realized with an Integrated Matching Circuit', , IEE Int. Conf. on Antennas and Propag., 2003, vol. 2, pp. 629-632.

[7.8] Catalá-Civera, J.M., Peñaranda-Foix, F., Sánchez-Hernández, D. y de los Reyes, E., 'Precise Dielectric Properties Determination of Laminar-Shaped Materials in a PartiallyFilled Waveguide', IEEE Antennas and Propagat. Society. Symp., 1999, pp. 1942-1945.

[7.9] Martínez-Vázquez M., y Litschke, O., 'Quadband Antenna for Handheld Personal Communications Devices', IEEE Antennas and Propagat. Society. Symp., 2003. 



\section{Capítulo 8: CONCLUSIONES Y LÍNEAS FUTURAS.}

Una síntesis vale por diez análisis.

Eugenio d' Ors.

\subsection{Conclusiones.}

El objetivo de esta tesis era el desarrollo de nuevos conceptos para antenas integradas en terminales de comunicaciones móviles, con vistas a la tercera generación. Se han analizado diferentes configuraciones de antenas que permiten operar en dos o más de las bandas de frecuencias reservadas par comunicaciones móviles.

Las antenas se basan en conceptos de parches en $\lambda / 4$ multiresonantes. En el caso de antenas duales, se recurría a parches cortocircuitados tipo PIFA con dos ramas resonantes, que permitían cubrir las bandas de GSM y DCS. Para obtener una tercera resonancia a la frecuencia de PCS sin deteriorar en demasía las características de los dos primeros modos se recurría a un parche parásito cortocircuitado, acoplado al parche prinipal, sin por ello aumentar el tamaño total de la estructura. Finalmente, se ha demostrado que es posible añadir un cuarto modo mediante el uso de una ranura de longitud $\lambda / 2$ para cubrir, por ejemplo, la banda de UMTS. Así, tienen el tamaño adecuado para que su integración en los terminales actuales sea posible.

Las estructuras aquí analizadas estaban, en su mayoría, inscritas en un área rectangular de pequeñas dimensiones. Pero se ha visto además que su geometría se podría adaptar a la forma del volumen destinado a la antena por los diseñadores de los terminales, sin que por ello se modificara su principio de funcionamiento. Se ha logrado, en definitiva, diseñar antenas de pequeño tamaño con buenas prestaciones, que sin embargo pueden ser mejoradas en algunos aspectos, como en el ancho de banda o los diagramas de radiación.

Se ha analizado también brevemente el efecto de los materiales empleados sobre el funcionamiento de las antenas. Este aspecto tiene una especial importancia si se tiene en cuenta que, a menudo, los parámetros de estos materiales son desconocidos por el diseñador, que debe recurrir a menudo al método de prueba y error.

El efecto de los materiales es especialmente significativo si se tiene en cuenta la creciente 
importancia dada a la eficiencia de las antenas. Ya que los terminales son cada vez más pequeños, y se quiere optimizar al máximo la energía disponible, es esencial aprovechar al máximo la potencia entregada a la antena. Así la caracterización de la eficiencia en una fase temprano del desarrollo permite disponer de un parámetro más para caracterizar el funcionamiento del sistema.

\subsection{LÍNEAS ABIERTAS.}

Los conceptos de antenas aquí presentados pueden ser adaptados también para operación con más bandas de frecuencias. Se podrían introducir así la banda de WLAN o de Bluetooth, para comunicaciones en entornos cerrados. En este caso quedaría por resolver el problema de la alimentación, puesto que los fabricantes tienden a utilizar para ello dos puertos distintos, debido al elevado precio de los duplexores necesarios si solamente se utiliza un puerto. Pueden aparecer entonces problemas de aislamiento y acoplamientos mutuos, que hay que tener en cuenta en el desarrollo.

Se ha comentado igualmente que, conforme aumenta el número de estándares que se pretende cubrir, aumenta también la fragilidad e inestabilidad del parche, por lo que se deben utilizar los medios de fijación adecuados. Una buena alternativa sería imprimir la antena sobre un substrato dieléctrico, por ejemplo una lámina de Duroid de $1 \mathrm{~mm}$ de espesor, de permitividad $\varepsilon_{\mathrm{r}}=2,2$ [8.1], tal y como se ha comentado en el apartado 7.4 y cuya implementación se muestra en la Figura 8.1.

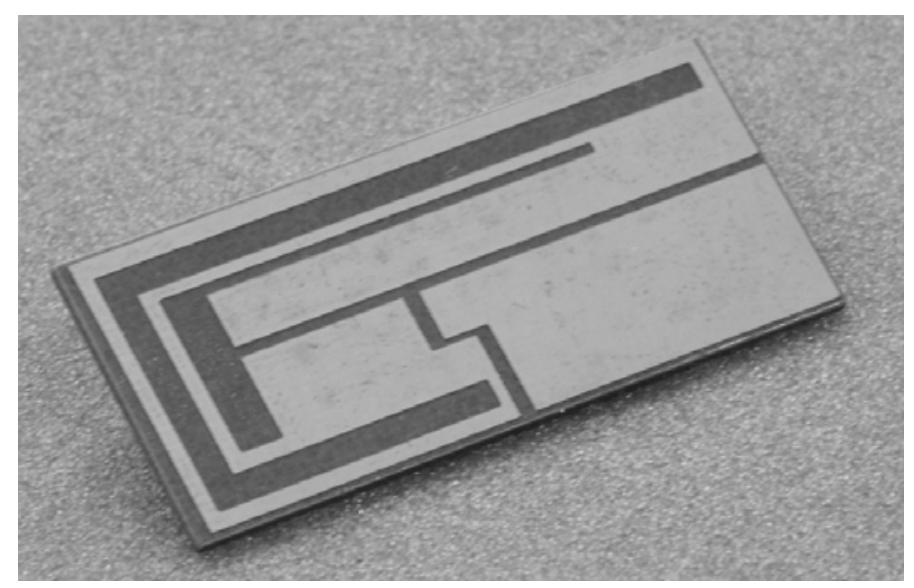

Figura 8.1: Antena MQ sobre substrato Duroid.

Se ha visto que el cambio de la permitividad relativa bajo el parche afecta al comportamiento de la antena, por lo que es necesario modificar la estructura, ajustando el ancho y largo de la ranura, y la separación entre elementos, para compensar los desplazamientos de las frecuencias de resonancia y compensar la desadaptación.

Otra opción plausible, para abaratar costes, sería imprimir la estructura metálica sobre un lámina flexible, como Polyimide o Poliéster [8.2]. Un ejemplo se muestra en la Figura 8.2. En este caso 
está aún por determinar la mejor manera de fijar la estructura para evitar arrugas u ondulaciones que afecten al funcionamiento final.

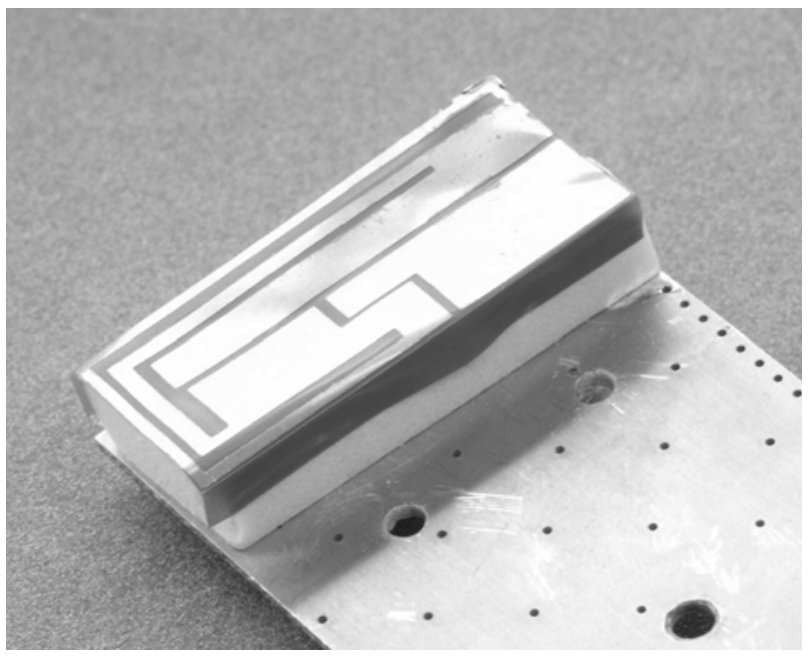

Figura 8.2: Antena MQ sobre substrato flexible.

En el Capítulo 2 se mencionaba también la posibilidad de utilizar el substrato cerámico LTCC para el desarrollo de antenas de comunicaciones móviles. Este substrato se ha utilizado con éxito en el IMST para fabricar módulos Bluetooth [8.3] o para aplicaciones de microondas [8.4], dando lugar a estructuras como las presentadas en la Figura 8.3. Con el soporte y la protección adecuada, podría utilizarse este material, junto con los conceptos aquí desarrollados, para determinados tipos de terminales.

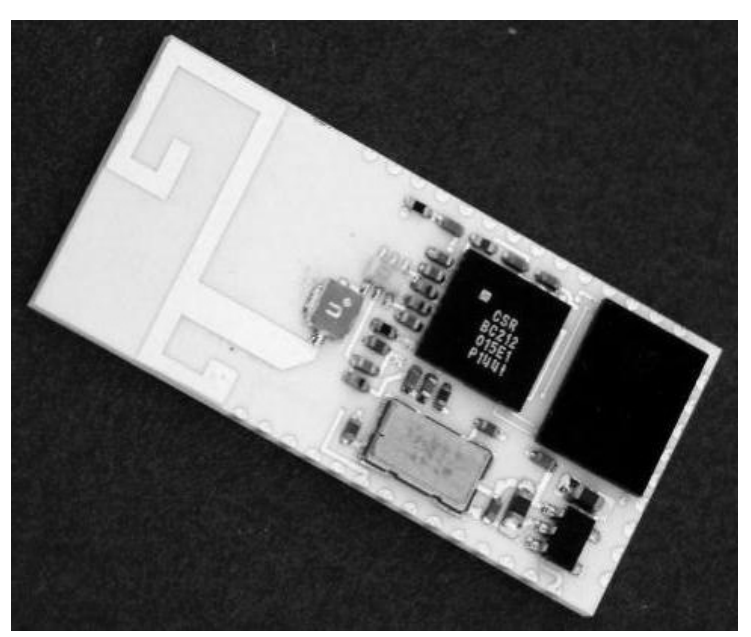

Módulo Bluetooth con antena integrada.

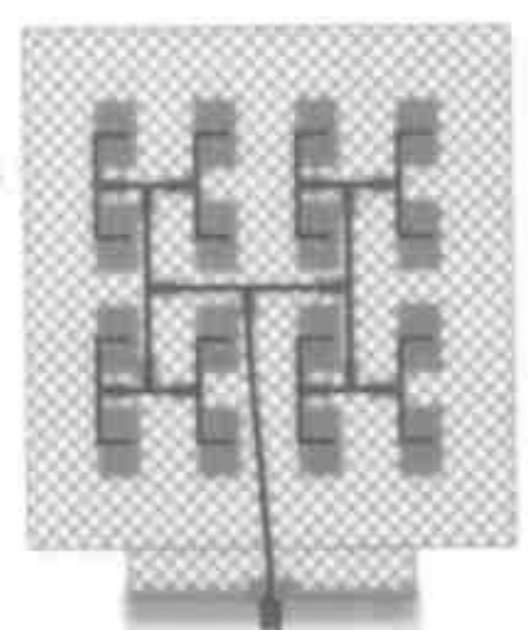

Array $4 \times 4$ a $24 \mathrm{GHz}$.

Figura 8.3: Antenas sobre LTCC.

Finalmente, cabe destacar que, con el desarrollo de nuevos y materiales y tecnologías, es posible que en algún momento no se distinga entre antena y soporte, y ambos formen un único elemento. Así, el uso de polímeros conductores permitiría integrar directamente la antena en la cubierta del terminal, en lo que se podría denominar smart casing (carcasa inteligente). Los conceptos de antenas aquí descritos seguirían siendo válidos, y bastaría con ajustar las dimensiones finales para 
lograr las frecuencias de funcionamiento deseadas. Esta tecnología se halla sin embargo en fase experimental.

Otro tema candente es el uso de interruptores de Sistema Micro-Electro-Mecánico (Micro-ElectroMechanical Systems, MEMS) en combinación con parches radiantes. Se podrían lograr así antenas reconfigurables, con agilidad en frecuencia y polarización. Esta tecnología se ha utilizado ya de forma experimental para determinadas aplicaciones de comunicaciones móviles de banda ancha [8.5] o para controlar la polarización [8.6].

\subsection{BibLIOGRAFÍA.}

[8.1] Martínez-Vázquez M., y Litschke, O., 'Quadband Antenna for Handheld Personal Communications Devices', IEEE Antennas and Propagat. Society. Symp., 2003.

[8.2] Nicholson, M., 'Flexible Printed Antennas', Microwaves \& RF, Noviembre 2002.

[8.3] Suárez-Pejenaute, E., Baggen, R. y Martínez-Vázquez, M., 'Integrated Antennas on LTCC Substrate for Bluetooth Applications', Journées Internationales de Nice sur les Antennes, 2002.

[8.4] Holzwarth, S., Kassner, J., Kulke, R. y Heberling, D., 'Planar Antenas on LTCCMultilayer Technology', IEE Int. Conf. on Antennas and Propag., 2001.

[8.5] Cetiner, B.A., Jofre, L., Chang, C.H., Qian, J.Y., Bachman, M., Li, G.P. y De Flaviis, F., 'Integrated MEM Antenna System for Wireless Communications', IEEE Microwave Theory \& Tech.. Int. Symp., 2002.

[8.6] Simons, R., Chun, D. y Katehi, L.P.B., 'Polarization Reconfigurable Patch Antenna using Microelectromechanical Systems (MEMS) Actuators', IEEE Antennas and Propagat. Society. Symp., 2002. 


\section{Anexo I. LISTA DE TABLAS E ILUSTRACIONES.}

\section{I.1. TABLAS.}

Capítulo 2: Antenas para terminales móviles.

Tabla 2.1: Ejemplos de tecnologías analógicas: redes de primera generación............................... 6

Tabla 2.2: Ejemplos de redes digitales: redes de segunda y tercera generación. ........................... 7

Tabla 2.3: Bandas de frecuencia de los sistemas de inalámbricos y celulares............................. 10

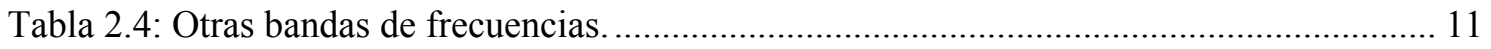

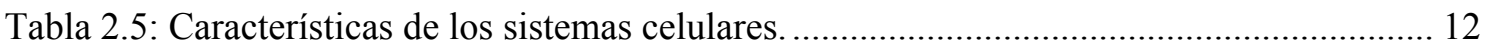

Tabla 2.6: Valores utilizados para las simulaciones de tejido humano........................................ 16

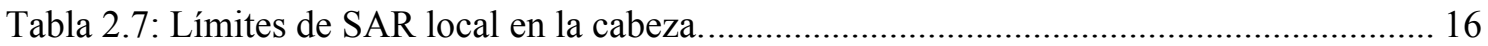

Capítulo 3: Introducción a las antenas impresas.

Tabla 3.1: Compromisos de diseño de un parche rectangular.................................................. 34

Capítulo 4: Métodos de análisis de antenas impresas.

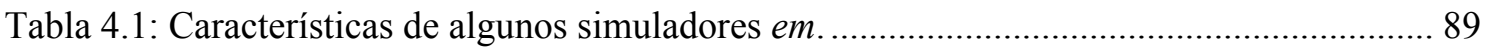

Capítulo 5: Antenas impresas de doble banda.

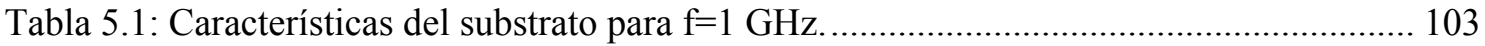

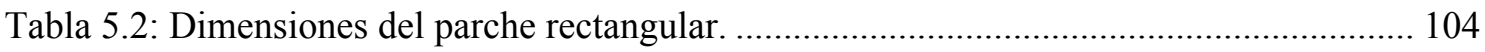

Tabla 5.3: Especificaciones de diseño del filtro espolón. .............................................................. 104

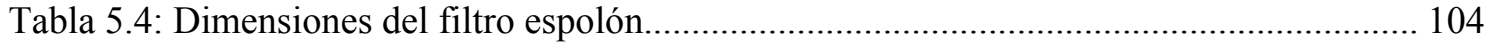

Tabla 5.5: Diagramas de radiación del parche rectangular con filtro espolón. .......................... 107

Tabla 5.6: Diagramas de radiación de la antena dual con filtro espolón.................................... 119

Capítulo 6: Antenas integradas multibanda.

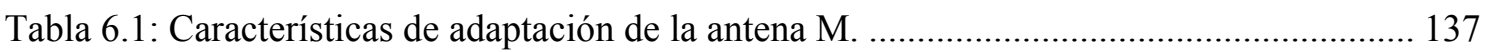




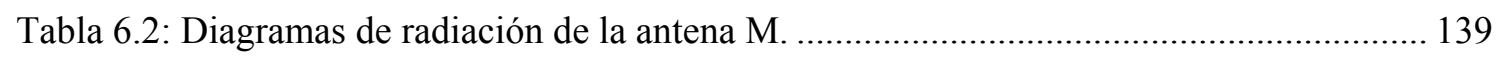

Tabla 6.3: Características de adaptación de la antena M con conector integrado...................... 141

Tabla 6.4: Diagramas de radiación de la antena M con conector integrado................................ 142

Tabla 6.5: Características de adaptación de la antena "ring-patch". ......................................... 144

Tabla 6.6: Características de adaptación de la antena de la antena "ring-patch" adaptada al terminal. 146

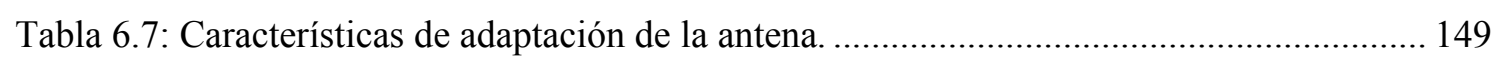

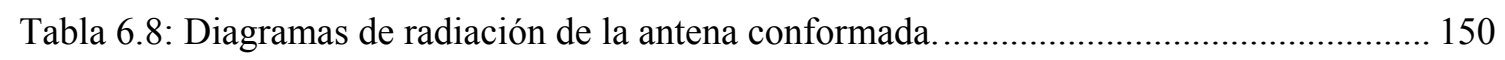

Tabla 6.9: Bandas de frecuencias asignadas a los principales estándares $(\mathrm{MHz})$...................... 151

Tabla 6.10: Características de adaptación de la antena de triple banda M3 .............................. 153

Tabla 6.11: Características de adaptación de la antena de triple banda MT............................... 157

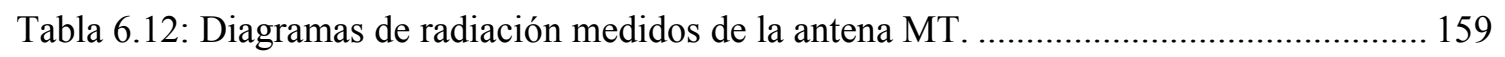

Capítulo 7: Influencia de los materiales.

Tabla 7.1: Características de los plásticos considerados para la fabricación del soporte. 174

\section{I.2. ILUSTRACIONES.}

Capítulo 2: Antenas para terminales móviles.

Figura 2.1: Evolución de la velocidad máxima de transmisión disponible. 11

Figura 2.2: Evolución de los terminales de comunicaciones móviles...................................... 13

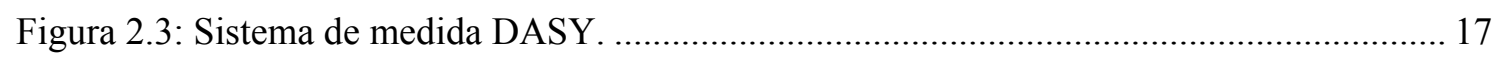

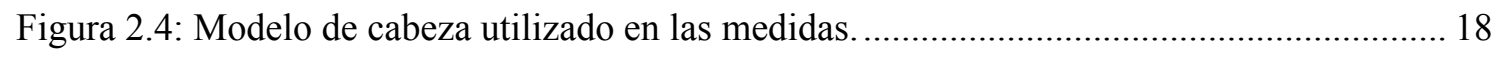

Figura 2.5: Dispositivo de medidas 3D del diagrama de radiación incluyendo el efecto del usuario.

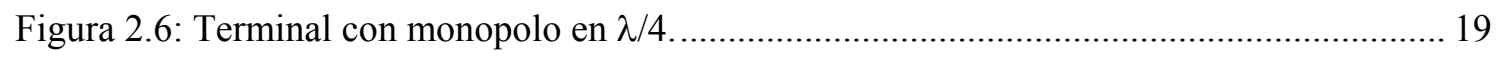

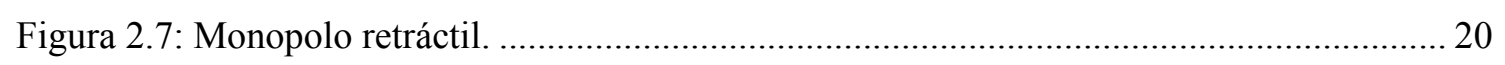

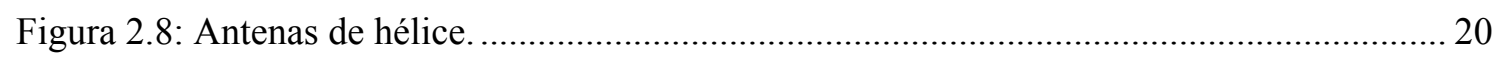

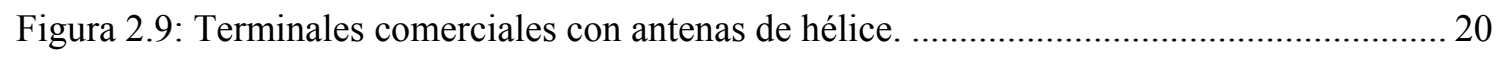

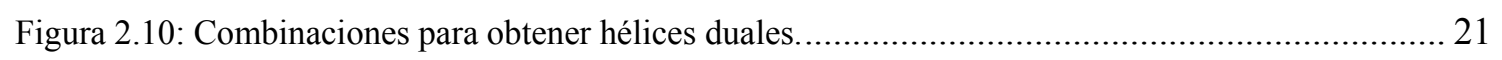


Figura 2.11: Terminal dual con combinación de hélice y monopolo. 21

Figura 2.12: Antena "meander line".

Figura 2.13: Ejemplos de equipos con antena "meander line" 22

Figura 2.14: Ejemplos de terminales con antena integrada. 22

Figura 2.15: Descripción del problema típico. 23

Figura 2.16: Antenas parche. 24

Figura 2.17: Terminales con antena integrada sobre soporte de plástico (a) o fijada a la cubierta posterior (b) 24

Figura 2.18: Antena impresa en substrato flexible sobre soporte de plástico. 24

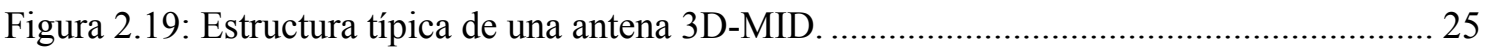

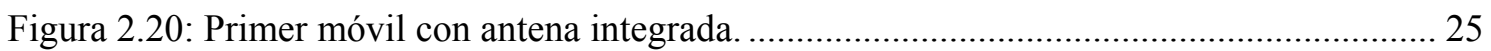

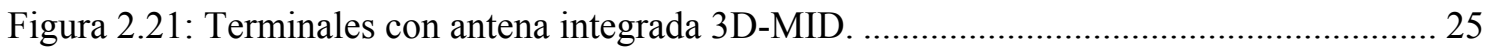

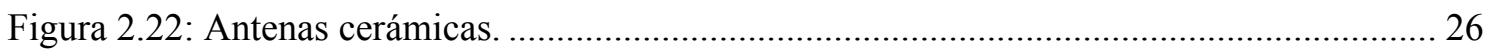

Figura 2.23: Módulo Bluetooth sobre LTCC con antena integrada........................................... 27

Capítulo 3: Introducción a las antenas impresas.

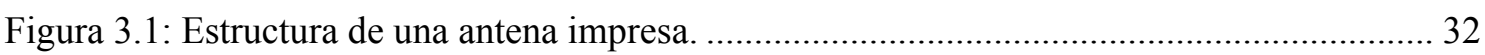

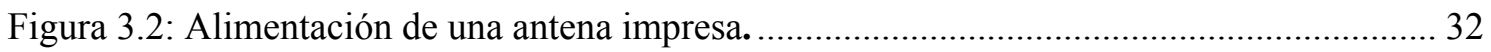

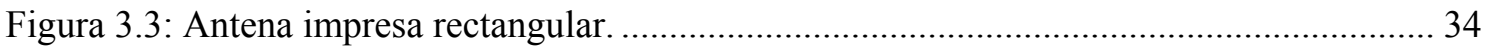

Figura 3.4: Antena alimentada por acoplamiento de apertura................................................... 37

Figura 3.5: Distribución de campos en un parche rectangular.................................................... 39

Figura 3.6: Límite teórico de Chu-Harrington: factor de calidad vs. tamaño de la antena, para antenas monomodo y diferentes valores de eficiencia.

Figura 3.7: Comparación de los límites teóricos obtenidos utilizando las fórmulas de ChuHarrington y de McLean................................................................................................... 50

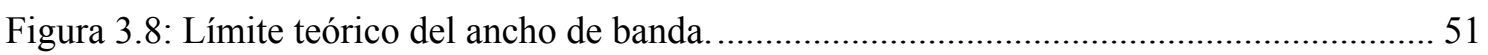

Figura 3.9: Límite teórico de la ganancia máxima alcanzable.................................................. 52

Capítulo 4: Métodos de análisis de antenas impresas.

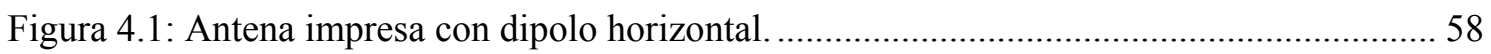

Figura 4.2: Modelo de la antena impresa como una malla de hilos. .......................................... 60

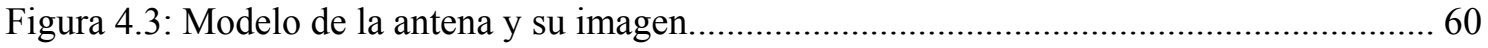




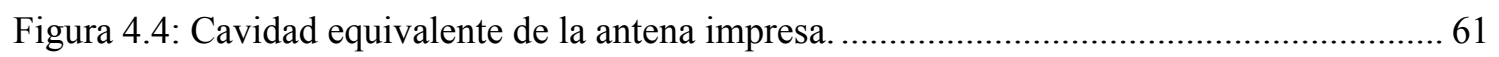

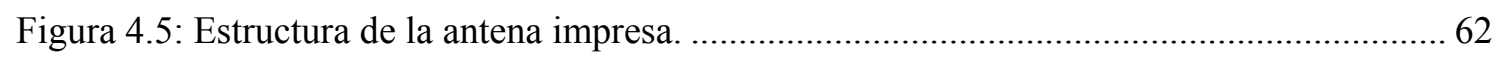

Figura 4.6: Campo eléctrico y distribuciones superficiales de corriente magnética en un parche rectangular.

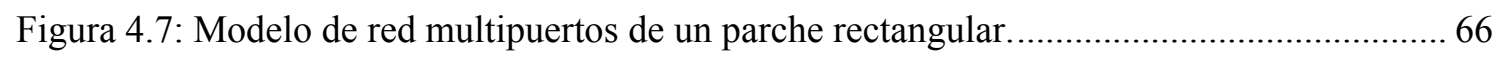

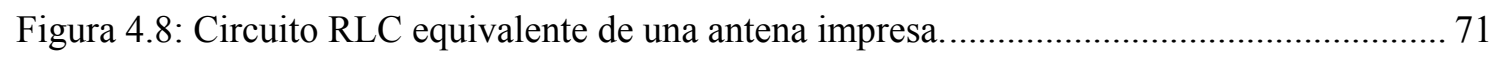

Figura 4.9: Modelo de línea de transmisión de una antena impresa rectangular......................... 72

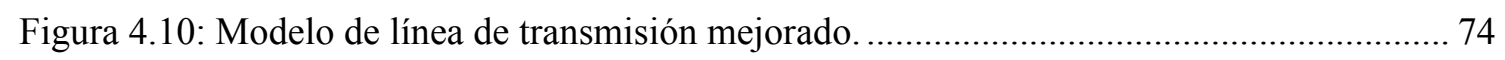

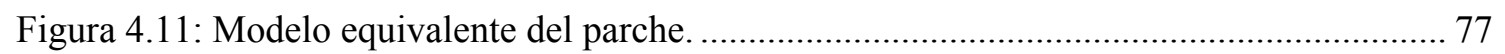

Figura 4.12: Descomposición del parche en los bordes. ……................................................... 81

Figura 4.13: Distribución de las componentes de campo en la celda de Yee (i=x, j=y, k=z)..... 84

Figura 4.14: Estructura de un nodo y numeración de los puertos en una celda TLM. ................. 86

Figura 4.15: Propagación de un pulso en una malla TLM bidimensional (corte 2D de una malla tridimensional).

Capítulo 5: Antenas impresas de doble banda.

Figura 5.1: Antena impresa dual rectangular con filtro espolón embedido.................................. 98

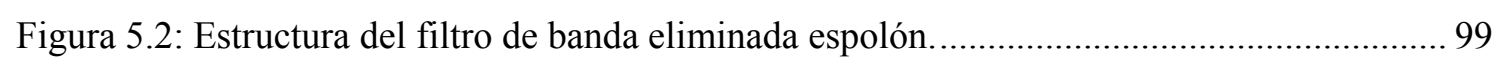

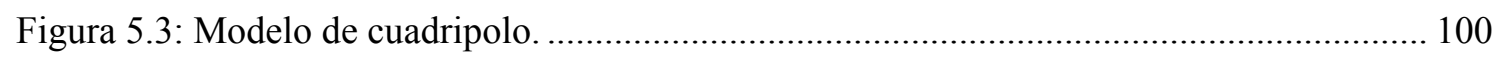

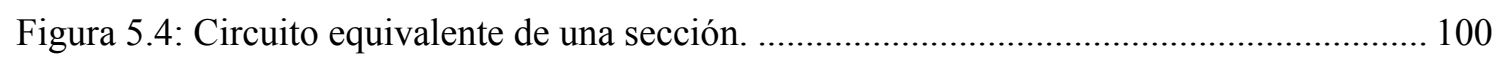

Figura 5.5: Circuito equivalente de la capacidad del hueco (gap capacity) de las líneas microtira

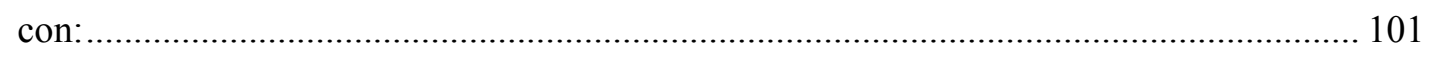

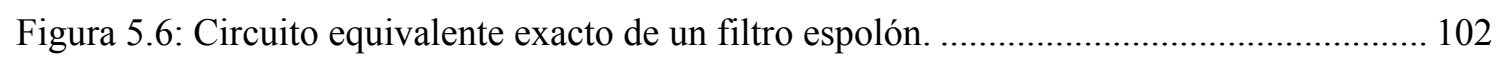

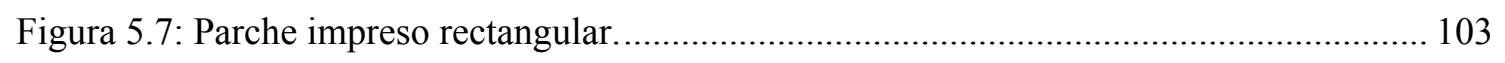

Figura 5.8: Estructura del parche dual con filtro espolón............................................................ 105

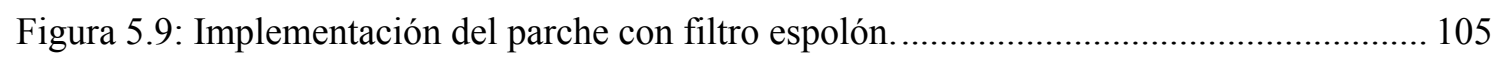

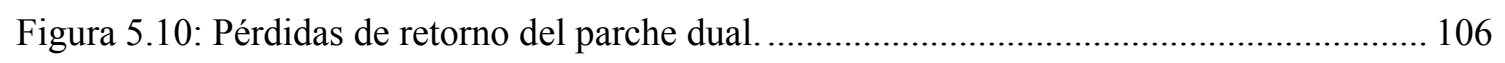

Figura 5.11: Distribuciones de campo eléctrico y diagramas de radiación del parche rectangular dual con filtro espolón en las bandas de GSM (izquierda) y DCS (derecha)...................... 106

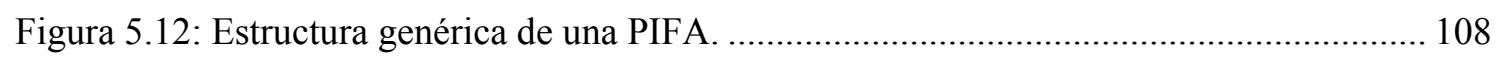

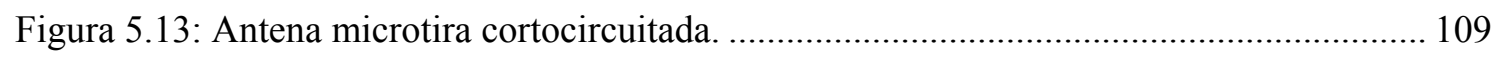


Figura 5.14: Estructura de una PIFA con cortocircuito menor que el ancho del parche. 109

Figura 5.15: Distribución de las componentes de campo eléctrico para diferentes anchos del cortocircuito, con $\mathrm{W}=\mathrm{L}$ 110

Figura 5.16: Distribución superficial de corriente en una PIFA para diferentes ratios W/L y diferentes anchos del cortocircuito.

Figura 5.17: Frecuencia de resonancia de una PIFA en función del tamaño del cortocircuito, $\mathrm{W}_{\mathrm{S}}$.

Figura 5.18: Ancho de banda de la PIFA en función de la altura del parche, para $\mathrm{W}_{\mathrm{S}}=\mathrm{W}$ y diferentes relaciones de aspecto $\mathrm{W} / \mathrm{L}$.

Figura 5.19: Ancho de banda de una PIFA para diferentes valores de $\mathrm{W}_{\mathrm{S}} / \mathrm{L}$, con $\mathrm{W}=\mathrm{L}$.

Figura 5.20: Descripción del problema típico

Figura 5.21: Representación gráfica de la esfera definida por McLean.

Figura 5.22: PIFA rectangular con filtro espolón, $\mathrm{W}=38 \mathrm{~mm}, \mathrm{~L}=13 \mathrm{~mm}, \mathrm{~h}=8 \mathrm{~mm}, \mathrm{~d}=5 \mathrm{~mm} \ldots \ldots .115$

Figura 5.23: Antena sobre el soporte metálico que simula el terminal.

Figura 5.24: PIFA con filtro espolón.

Figura 5.25: Pérdidas de retorno de la antena dual con filtro espolón.

Figura 5.26: Distribución de campo eléctrico (izquierda) y magnético (derecha) en la banda de GSM.

Figura 5.27: Distribución de campo eléctrico (izquierda) y magnético (derecha) en la banda de

DCS

Figura 5.28: Diagrama de radiación en las bandas de GSM (izquierda) y DCS (derecha). 118

Figura 5.29: Sistema de coordenadas utilizado en las medidas de los diagramas de radiación. 118

Figura 5.30: Circuito equivalente serie de una antena monobanda sobre un plano de masa. ... 120

Figura 5.31: Circuito equivalente paralelo de una antena monobanda sobre un plano de masa.120

Figura 5.32: Estructura de doble banda sobre plano de masa. 120

Figura 5.33: Pérdidas de retorno para diferentes longitudes del terminal (del PCB). 121

Figura 5.34: Ancho de banda en función del tamaño del terminal para las bandas de frecuencias de GSM y DCS. 121

Figura 5.35: Distribución de campo eléctrico para diferentes longitudes del PCB en la banda de GSM. 122

Figura 5.36: Distribución de campo eléctrico para diferentes longitudes del PCB en la banda de 
DCS.

Figura 5.37: Modelo del terminal con batería. 123

Figura 5.38: Pérdidas de retorno simuladas en función de la posición de la batería.

Figura 5.39: Ancho de banda relativo en función de la distancia de la antena a la batería.

Figura 5.40: Distribución del campo eléctrico en un plano perpendicular al terminal para diferentes posiciones de la batería. Banda de GSM.

Figura 5.41: Distribución del campo eléctrico en un plano perpendicular al terminal para diferentes posiciones de la batería. Banda de DCS...... 125

Figura 5.42: Volumen destinado a la antena (en rojo) en un terminal típico. 125

Figura 5.43: Posición de la antena respecto al PCB del terminal. 126

Figura 5.44: Pérdidas de retorno diferentes valores de $h_{1}$. 126

Figura 5.45: Frecuencia de resonancia en función de $h_{1}$, la altura de la antena en la parte superior del terminal $\left(\mathrm{h}_{2}=8 \mathrm{~mm}\right)$

Figura 5.46: Ancho de banda relativo en función de $\mathrm{h}_{1}\left(\mathrm{~h}_{2}=8 \mathrm{~mm}\right)$. 127

Figura 5.47: Distribución del campo eléctrico en un corte perpendicular al plano de masa para la banda de GSM. Izquierda: $h_{1}=h_{2}=8 \mathrm{~mm}$, derecha: $h_{1}=2 \mathrm{~mm}, h_{2}=8 \mathrm{~mm}$. 128

Figura 5.48: Distribución del campo eléctrico en un corte perpendicular al plano de masa para la banda de DCS. Izquierda: $h_{1}=h_{2}=8 \mathrm{~mm}$, derecha: $h_{1}=2 \mathrm{~mm}, h_{2}=8 \mathrm{~mm}$. 128

Figura 6.1: Estructura de la PIFA triangular con filtro espolón. 134

Figura 6.2: PIFA triangular con filtro espolón. 134

Figura 6.3: Pérdidas de retorno de la PIFA triangular con filtro espolón. 135

Figura 6.4: Distribución de campo eléctrico (izquierda) y magnético (derecha) de la antena triangular en la banda de GSM. 135

Figura 6.5: Distribución de campo eléctrico (izquierda) y magnético (derecha) de la antena triangular en la banda de DCS. 135

Figura 6.6: Diagrama de radiación de la antena triangular en las bandas de GSM (izquierda) y DCS (derecha)...

Figura 6.7: Geometría de la antena M......

Figura 6.8: Pérdidas de retorno medidas de la antena M.

Figura 6.9: Distribución de campo eléctrico (izquierda) y magnético (derecha) de la antena $\mathrm{M}$ en la banda de GSM. 137 
Figura 6.10: Distribución de campo eléctrico (izquierda) y magnético (derecha) de la antena M en la banda de DCS.

Figura 6.11: Diagrama de radiación de la antena $\mathrm{M}$ en las bandas de GSM (izquierda) y DCS (derecha). 138

Figura 6.12: Sistema de coordenadas utilizado en las medidas de los diagramas de radiación. 138

Figura 6.13: Estructura de la antena $\mathrm{M}$ con conector integrado. 140

Figura 6.14: Antena $\mathrm{M}$ con conector integrado. 140

Figura 6.15: Pérdidas de retorno medidas, con y sin usuario, de la antena $\mathrm{M}$ con conector integrado.

Figura 6.16: Estructura de la antena "ring-patch".

Figura 6.17: Antena "ring-patch"

Figura 6.18: Pérdidas de retorno de la antena "ring-patch".

Figura 6.19: Distribución de campo eléctrico (izquierda) y magnético (derecha) de la antena "ringpatch" en la banda de GSM.

Figura 6.20: Distribución de campo eléctrico (izquierda) y magnético (derecha) de la antena "ringpatch" en la banda de DCS.

Figura 6.21: Diagrama de radiación de la antena "ring-patch" en las bandas de GSM (izquierda) y DCS (derecha).

Figura 6.22: Estructura de la antena "ring-patch" adaptada al terminal.

Figura 6.23: Maqueta del terminal con la antena integrada.

Figura 6.24: Pérdidas de retorno de la antena "ring-patch" adaptada a la forma del terminal.. 147

Figura 6.25: Esquema de construcción de la antena conformada. 147

Figura 6.26: Modelo de simulación de una antena de doble banda adaptada a la cubierta del terminal. 148

Figura 6.27: Antena sobre soporte de plástico. 148

Figura 6.28: Pérdidas de retorno medidas de la antena de doble banda adaptada a la cubierta del terminal.

Figura 6.29: Distribución de las bandas de frecuencia (en MHz) de los principales estándares.151

Figura 6.30: Estructura de la antena de triple banda M3 con parche parásito cortocircuitado.. 153

Figura 6.31: Antena M3.

Figura 6.32: Pérdidas de retorno medidas de la antena de triple banda M3 154 
Figura 6.33: Distribución de campo eléctrico (izquierda) y magnético (derecha) de la antena M3 en la banda de GSM...... 154

Figura 6.34: Distribución de campo eléctrico (izquierda) y magnético (derecha) de la antena M3 en la banda de DCS... 155

Figura 6.35: Distribución de campo eléctrico (izquierda) y magnético (derecha) de la antena M3 en la banda de PCS. 155

Figura 6.36: Diagrama de radiación de la antena M3 en las bandas de GSM (izquierda), y DCS (centro) y PCS (derecha)... 155

Figura 6.37: Modelo de terminal con antena integrada de triple banda MT. 156

Figura 6.38: Antena de triple banda MT. 156

Figura 6.39: Simulación y medidas de las pérdidas de retorno de la antena de triple banda MT.157

Figura 6.40: Distribución de campo eléctrico (izquierda) y magnético (derecha) de la antena MT en la banda de GSM. 157

Figura 6.41: Distribución de campo eléctrico (izquierda) y magnético (derecha) de la antena MT en la banda de DCS... 158

Figura 6.42: Distribución de campo eléctrico (izquierda) y magnético (derecha) de la antena MT en la banda de PCS. . 158

Figura 6.43: Diagrama de radiación de la antena MT en las bandas de GSM (izquierda), y DCS (centro) y PCS (derecha). 158

Figura 6.44: Modelo de la antena de cuatro bandas MQ integrada en el terminal. 160

Figura 6.45: Antena MQ. 160

Figura 6.46: Simulación y medidas de la antena de cuatro bandas MQ. 161

Figura 6.47: Distribución de campo eléctrico (izquierda) y magnético (derecha) de la antena MQ en la banda de GSM. 161

Figura 6.48: Distribución de campo eléctrico (izquierda) y magnético (derecha) de la antena MQ en la banda de DCS.. 162

Figura 6.49: Distribución de campo eléctrico (izquierda) y magnético (derecha) de la antena MQ en la banda de PCS. 162

Figura 6.50: Distribución de campo eléctrico (izquierda) y magnético (derecha) de la antena MQ en la banda de UMTS. 162

Figura 6.51: Diagrama de radiación de la antena MQ en las bandas de GSM (izquierda), y DCS (centro) y PCS (derecha). 163 
Figura 6.52: Diagrama de radiación de la antena MQ en la banda de UMTS. 163

Capítulo 7: Influencia de los materiales.

Figura 7.1: Antena parche genérica integrada en un terminal. 165

Figura 7.2: Circuito equivalente serie (Thévenin) de una antena.

Figura 7.3: Circuito equivalente de las pérdidas de la antena.

Figura 7.4: Circuito equivalente de las pérdidas de la antena en la cavidad. 168

Figura 7.5: Determinación de los parámetros $\Delta \mathrm{s}_{\max }$ y $\Delta \mathrm{s}_{\min }$ a partir de las medidas.

Figura 7.6: Cavidad con cortocircuito deslizante utilizada en las medidas. 169

Figura 7.7: Modelo de la antena MQ con cubierta de plástico 170

Figura 7.8: Pérdidas de retorno medidas de la antena sólo y con cubierta de plástico.

Figura 7.9: Eficiencia de radiación, eficiencia total y pérdidas de retorno de la antena sin cubierta de plástico (izquierda) y con cubierta (derecha) para la banda de GSM. 172

Figura 7.10: Eficiencia de radiación, eficiencia total y pérdidas de retorno de la antena sin cubierta de plástico (izquierda) y con cubierta (derecha) para las bandas de DCS, PCS y UMTS. 172

Figura 7.11: Modelo de simulación de la antena M con soporte de plástico. 173

Figura 7.12: Pérdidas de retorno simuladas, para soportes de con diferentes materiales plásticos comerciales. 174

Figura 7.13: Distribución de campo eléctrico en la banda de GSM para la antena sin soporte dieléctrico (izquierda) y con soporte de Fortron ${ }^{\circledR}$ (derecha). 175

Figura 7.14: Distribución de campo eléctrico en la banda de DCS para la antena sin soporte dieléctrico (izquierda) y con soporte de Fortron ${ }^{\circledR}$ (derecha). 175

Figura 7.15: Pérdidas de retorno de la antena sin soporte dieléctrico y con soporte de Teflón. 176

Figura 7.16: Distribución de campo eléctrico en la banda de GSM para la antena sin soporte dieléctrico (izquierda) y con soporte de Teflón (derecha). 176

Figura 7.17: Distribución de campo eléctrico en la banda de DCS para la antena sin soporte dieléctrico (izquierda) y con soporte de Teflón (derecha). 176

Figura 7.18: Distribución de campo eléctrico en la banda de PCS para la antena sin soporte dieléctrico (izquierda) y con soporte de Teflón (derecha). 177

Figura 7.19: Distribución de campo eléctrico en la banda de UMTS para la antena sin soporte dieléctrico (izquierda) y con soporte de Teflón (derecha). 177

Figura 7.20: Antena M de doble banda con conector integrado. 177 
Figura 7.21: Eficiencia de la antena con soporte Vectra ${ }^{\circledR}$ y parche de latón.............................. 178

Figura 7.22: Eficiencia de la antena con soporte Vectra ${ }^{\circledR}$ y parche de acero. ........................... 178

Figura 7.23: Eficiencia de la antena con soporte Topas ${ }^{\circledR}$ y parche de acero............................. 179

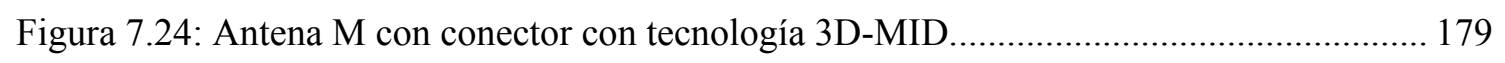

Figura 7.25: Eficiencia de la antena con soporte Vectra ${ }^{\circledR}$ metalizado (MID). .......................... 180

Capítulo 8: Conclusiones y líneas futuras.

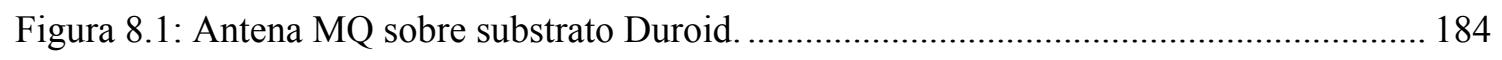

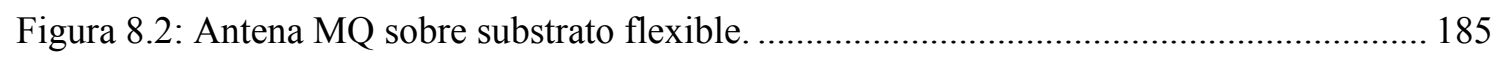

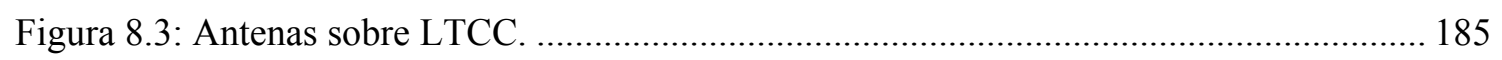




\section{Anexo II. Glosario de ABREVIATURAs.}

AMPS: Advanced Mobile Phone System.

ANSI : American National Standards Institute.

CAD: Computed Aided Design, Diseño Asistido por Ordenador.

CDMA: Code Division Multiple Access, Acceso Múltiple por División de Código.

CENELEC: Comité Européen de Normalisation Electrotechnique.

CG-FFT: Conjugate Gradient-Fast Fourier Transform, Gradiente Conjugado-Transformada Rápida de Fourier.

D-AMPS: Digital AMPS, ver AMPS.

DASY : Dosimetric Assessment System.

DCS: Digital Cellular System, GSM 1800.

DECT: Digital Enhanced Cordless Telecommunications.

EAN: Edge Admitance Network, Red de Admitancias de Borde.

EDGE: Enhanced Data Ranges for GSM Evolution, ver GSM y E-GSM.

E-GSM: Extended GSM, ver EDGE y GSM.

EM: Electro-Magnético.

EMPIRE: Electro Magnetic field solver for the analysis of Packages, Interconnects, Radiators, and waveguides Elements.

ETSI: European Telecommunications Standards Institute.

FCC: Federal Communication Commission.

FDD : Frequency Division Duplex, Duplex por División en Frecuencia.

FDMA: Frequency Divition Multiple Access. Acceso Múltiple por División en Frecuencia.

FDTD: Finite Difference-Time Domain, Diferencias Finitas en el Dominio Temporal.

FEM: Finite Element Meted, Método de los Elementos Finitos.

FFT: Fast Fourier Transform, Transformada Rápida de Fourier. 
FITD: Finite Integration-Time Domain, Integración Finita en el Dominio Temporal.

GPRS: General Packet Radio Service.

GPS: Global Positioning System.

GSM 1800: ver DCS.

GSM 900: ver GSM, E-GSM.

GSM: Global System for Mobile Communications.

HSCSD: High Speed Circuit Switch Data.

ICNIRP: International Commission on Non-Ionizing Radiation Protection.

Inmarsat: INternational MARtime SATellite system.

IEEE : Institute of Electrical and Electronics Engineers.

IFA : Inverted-F Antenna, Antena en F Invertida.

IMT-2000: International Mobile Telecommunications - 2000.

ISM: Industrial, Scientific and Medical, banda de frecuencias para aplicaciones Industriales Científicas y Médicas.

LAN: Local Area Network, Red de Área Local.

LTCC: Low Temperature Co-fired Ceramics.

MBS: Mobile Broadband Systems.

MCN Mutual Coupling Network, Red de Acoplamientos Mutuos.

MEMS: Micro-Electro-Mechanical Systems.

MMS: Multimedia Message Service.

MoM: Method of Moments, Método de los Momentos.

MPE: Mixed Potential Equation, Ecuación de Potencial Mixto.

MPIE: Mixed Potential Integral Equation, Ecuación Integral de Potencial Mixto.

MSA : Microstrip Short-circuited Antena, Antena Microtira Cortocircuitada.

N-AMPS: Narrowband Advanced Mobile Phone System, ver AMPS.

N-CDMA: Narrowband CDMA, ver CDMA.

NMT450: Nordic Mobile Telephones.

NTT: Nippon Telegraph and Telephone.

PCB : Printed Circuit Board, Placa de Circuito Impreso. 
PCMCIA : Personal Computer Memory Card International Association.

PCS: Personal Communications System.

PDC: Personal Digital Cellular System.

PDC-P: Personal Digital Cellular System - Packet system.

PHS: Personal Handyphone System.

PIFA : Planar Inverted-F Antenna, Antena en F Invertida Plana.

PML: Perfectly Matched Layer.

RF: Radio Frecuencia.

ROE: Relación de Onda Estacionaria, ver VSWR.

SAR : Specific Absorption Rate, Tasa de Absorbción Específica.

SDA: Spectral Domain Analysis, Análisis en el Dominio Espectral.

SMD: Surface Mounted Devices.

SMS: Short Message Service.

TDD : Time Division Duplex, Duplex por División en el Tiempo.

TDMA: Time División Multiple Access, Acceso Múltiple por División en el Tiempo.

TE: Transversal Eléctrico.

TEM : Transversal Electro-Magnético.

TETRA: TErrestrial Trunked Radio.

TLM: Transmisión Line Matrix, Matriz de Líneas de Transmisión.

TM: Transversal Magnético.

UIT : Unión Internacional de Telecomunicaciones.

UMTS: Universal Mobile Telecommunications System.

VSWR: Voltage Standing Wave Ratio, ver ROE.

W-CDMA: Wideband CDMA, ver CDMA.

WLAN: Wireless Local Area Network, Red de Área Local Inalámbrica. 



\section{Anexo III. PUblicaciones Y PATENTES.}

\section{III.1. Publicaciones en ReVistas internacionales.}

@ A. Martínez-González, M.A. Sánchez-Aguilar, M. Martínez-Vázquez y D. SánchezHernández, 'A Comparison of Dual-Band Spur-Line Printed Antennas for Hand-Held Handsets', Microwave and Optical Technology Lett., 2001, vol. 30, n 3, pp. 205-207.

\& M. Martínez-Vázquez, M. Geissler, D. Heberling y D. Sánchez-Hernández, 'Compact Dual-Band Antenna for Mobile Handsets', Enero 2002, Microwave and Optical Technology Lett., vol. 32, $\mathrm{n}^{\circ} 2$, pp. 87-88.

\& M. Martínez-Vázquez, O. Litschke, D. Heberling y D. Sánchez-Hernández, 'On the Design of Integrated Multiband Antennas for Personal Communications Handsets', Submitted to IEE Proceedings.

\section{III.2. Publicaciones en Conferencias internacionales.}

œ M. Martínez-Vázquez, A. Martínez-González, E. Ávila-Navarro, D. Sánchez-Hernández, 'Dual-Band Dual-Mode Printed Bow-Tie Antennas Integrated in Hand-Held Terminals for Wireless Communications', 28th European Microwave Conf., Ámsterdam, Octubre 1998.

\& M. Martínez-Vázquez, D. Sánchez-Hernández, 'Design and Troubleshooting of Microstrip Antennas Using Commercial Electromagnetic Simulators', 7th International Symp. on Recent Advances in Microwave Technology ISRAMT’99, Málaga, Diciembre 1999.

\. Martínez-Vázquez y D. Sánchez-Hernández, 'Benchmarking of Commercial Electromagnetic Simulators for the Design of Printed Antennas', Millennium Conf. on Antennas and Propagation AP2000, Davos (Suiza), Abril 2000.

@ M. Martínez-Vázquez, J.M. Catalá-Civera y D. Sánchez-Hernández, 'Quantification of Errors in the Prediction of Resonant Frequencies for Printed Antennas using Commercial Electromagnetic Simulators through the Utilisation of Measured Substrate Dielectric 
Properties', Millennium Conf. on Antennas and Propagation AP2000, Davos (Suiza), Abril 2000.

- M. Martínez-Vázquez y D. Sánchez-Hernández, 'Design of a Compact Dual-Band Antenna for Mobile Communications Handsets', $30^{\text {th }}$ European Microwave Conf., París (Francia), Octubre 2000.

\& M. Martínez-Vázquez, M. Geissler y D. Heberling, 'Volume Considerations in the Design of Dual-Band Handset Antennas', IEEE Antennas and Propagat. Society. Symp., Boston (Massachussets), Julio 2001.

- M. Martínez-Vázquez, M. Geissler y D. Heberling, 'Volume Reduction of Integrated Handset Antennas', $16^{\text {th }}$ International Conf. on Applied Electromagnetics and Communications, Dubrovnik (Croacia), Octubre 2001.

\$ M. Martínez-Vázquez y O. Litschke, 'Novel Triple-Band Antennas for Personal Communicatons Handsets', IEEE Antennas and Propagat. Society. Symp., San Antonio (Texas), Junio 2002.

\ M. Martínez-Vázquez y O. Litschke, 'Design Considerations for Quad-Band Antennas Integrated in Personal Communications Devices', Journées Internationales de Nice sur les Antennes, Niza (Francia), Noviembre 2002.

\& E. Suárez-Pejenaute, R. Baggen y M. Martínez-Vázquez, 'Integrated Antennas on LTCC Substrate for Bluetooth Applications', Journées Internationales de Nice sur les Antennes, Niza (Francia), Noviembre 2002.

œ M. Martínez-Vázquez y O. Litschke, 'Quadband Antenna for Handheld Personal Communications Devices', IEEE Antennas and Propagat. Society. Symp., Columbus (Ohio), Junio2003.

@ M. Martínez-Vázquez y O. Litschke y D. Heberling, 'Design of a Quadband Antenna System for PCMCIA', Submitted to the International ITG Conf. on Antennas, Berlín (Alemania), Septiempbre 2003.

$\infty$ O. Litschke, M. Geissler, M. Martínez-Vázquez y D. Heberling, 'Adaptation of the Wheeler-Cap Method for Measuring the Efficiency of Mobile Handset Antennas', Submitted to the International ITG Conf. on Antennas, Berlín (Alemania), Septiempbre 2003.

œ D. Heberling, M. Geissler, O. Litschke y M. Martínez-Vázquez, 'Improved Radiation Efficiency of Mobile Systems and Application to a Novel Multiband Antenna', International Microwave and Optoelectronics Conf., Iguazú (Brasil), Septiembre 2003.

œ M. Martínez-Vázquez y O. Litschke, 'Small Multiband Antenna for Personal Communications Devices', Submitted to the $17^{\text {th }}$ International Conf. on Applied 
Electromagnetics and Communications, Dubrovnik (Croacia), Octubre 2003.

\section{III.3. Publicaciones en conferencias nacionales.}

- M. Martínez-Vázquez, E. de los Reyes y D. Sánchez-Hernández, 'Antenas Integradas en Terminales de Comunicaciones Móviles', Simposium Nacional de la URSI, Santiago de Compostela, Septiembre 1999.

E. Suárez-Pejenaute, R. Baggen y M. Martínez-Vázquez, 'Antenas Integradas para Aplicaciones Bluetooth sobre Substrato LTCC', Simposium Nacional de la URSI, Alcalá de Henares, Septiembre 2002.

\section{III.4. OtRAS PUBLICACIONES.}

๑ M. Martínez-Vázquez, A. Martínez-González, E. Ávila-Navarro, D. Sánchez-Hernández, 'Dual-Band Printed Bow-Tie Antennas for Hand-Held Wireless Terminals', COST 260 Meeting on Smart Antennas, Trondheim (Noruega), 1998.

œ M. Martínez-Vázquez, J.M. Catalá-Civera y D. Sánchez-Hernández, 'Effect of Measured Substrate Dielectric Properties on the Prediction of Patch Antenna Resonant Frequencies using Commercial Electromagnetic Simulators', COST 260 Meeting on Smart Antennas, Wroclaw (Polonia), 1999

ฯ M. Martínez-Vázquez y D. Sánchez-Hernández, 'Integrated Antennas for Mobile Communication Handsets', COST 259 Meeting on Wireless Flexible Personalized Communications, Valencia, 2000.

\& M. Martínez-Vázquez, M. Geissler, D. Heberling y D. Sánchez-Hernández, 'Recent Developments in Antenna Design for Personal Communications Handsets at the IMST', COST 260 Meeting on Smart Antenas, Rennes (Francia), 2000.

œ M. Martínez-Vázquez, M. Geissler, D. Heberling y D. Sánchez-Hernández, 'Dualband Spurline-Antenne für Handys', ITG Diskussionssitzung: Antennen für mobile Systeme 2000, Starnberg (Alemania), 2000.

œ M. Geissler, M. Martínez-Vázquez, D. Manteuffel, D. Heberling e I. Wolf, 'Bandbreitenuntersuchungen an integrierten Handyantennen', ITG Diskussionssitzung: Antennen für mobile Systeme 2000, Starnberg (Alemania), 2000. 
๑ M. Martínez-Vázquez, M. Geissler y D. Heberling, 'Advanced Antenna Concepts for PCS Handsets', COST 260 Meeting on Smart Antennas, Goteborg (Suecia), 2001.

@ M. Martínez-Vázquez, O. Litschke y D. Heberling, 'Design of Integrated Multiband Antennas for Personal Communications Handsets', COST 284 Meeting on Innovative Antennas for Emerging Terrestrial \& Space-based Applications, Budapest (Hungría), 2003.

\section{III.5. PATENTES.}

٪ D. Sánchez Hernández, M. Martínez Vázquez, L. Jianying y E. de los Reyes, 'Antena Impresa de Banda Dual', Patente Española ES 2156832 A1, Oficina Española de Patentes y Marcas, 2002.

๑ D. Sánchez Hernández y M. Martínez Vázquez, ‘Antena Dual para Terminales Móviles', $\mathrm{n}^{\circ}$ de solicitud P-200002704, Oficina Española de Patentes y Marcas, 2000.

- M. Martinez-Vazquez y M. Geissler, 'Planare Mobilfunkantenne', Patente Alemana DE 10054 192, Deutschen Patent- und Markenamt, Alemania, 2002.

s M. Geissler y M. Martinez-Vazquez, 'Mehrbandantenne mit parasitären Strahlern', ${ }^{\circ}$ de solicitud 10204 079.6, Deutschen Patent- und Markenamt, Alemania, 2002 (concesión prevista en Agosto 2003).

- M. Geissler, M. Martinez-Vazquez y A. Winkelmann, 'Antenne mit geneigter Strahlerfläche', n de solicitud 10231919.7, Deutschen Patent- und Markenamt, 2002 (publicación prevista en Enero 2004). 